\title{
Laser-Induced Back- Ablation of Aluminum Thin Films using Picosecond Laser Pulses
}

\author{
A.B. Bullock \\ Doctor of Philosopy
}

May 26, 1999

U.S. Department of Energy

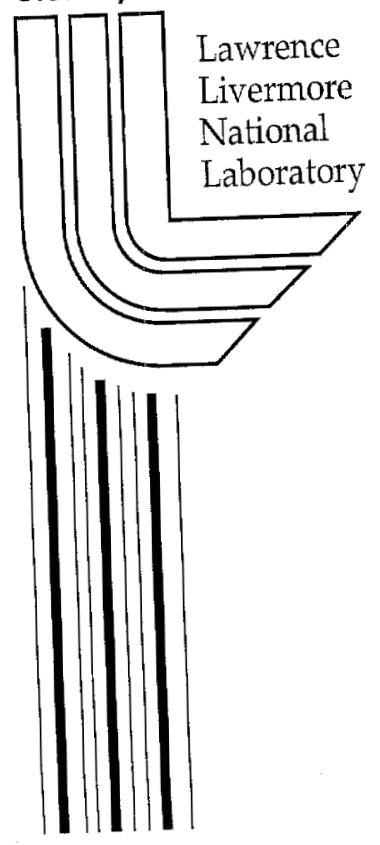




\section{DISCLAIMER}

This document was prepared as an account of work sponsored by an agency of the United States Government. Neither the United States Government nor the University of California nor any of their employees, makes any warranty, express or implied, or assumes any legal liability or responsibility for the accuracy, completeness, or usefulness of any information, apparatus, product, or process disclosed, or represents that its use would not infringe privately owned rights. Reference herein to any specific commercial product, process, or service by trade name, trademark, manufacturer, or otherwise, does not necessarily constitute or imply its endorsement, recommendation, or favoring by the United States Government or the University of California. The views and opinions of authors expressed herein do not necessarily state or reflect those of the United States Government or the University of California, and shall not be used for advertising or product endorsement purposes.

This work was performed under the auspices of the U. S. Department of Energy by the University of California, Lawrence Livermore National Laboratory under Contract No. W-7405-Eng-48.

This report has been reproduced directly from the best available copy.

Available electronically at http://www.doe.gov/bridge

Available for a processing fee to U.S. Department of Energy and its contractors in paper from

U.S. Department of Energy

Office of Scientific and Technical Information

P.O. Box 62

Oak Ridge, TN 37831-0062

Telephone: (865) 576-8401

Facsimile: (865) 576-5728

E-mail: reports@adonis.osti.gov

Available for the sale to the public from

U.S. Department of Commerce

National Technical Information Service 5285 Port Royal Road

Springfield, VA 22161

Telephone: (800) 553-6847

Facsimile: (703) 605-6900

E-mail: orders@ntis.fedworld.gov

Online ordering: http://www.ntis.gov/ordering.htm

OR

Lawrence Livermore National Laboratory

Technical Information Department's Digital Library

http://www.Ilnl.gov/tid/Library.html 
Laser-Induced Back-Ablation of Aluminum Thin Films Using Picoseond Laser Pulses

By

ANTHONY BURLINGAME BULLOCK

B.S. (University of California, Davis) 1993

M.S. (University of California, Davis) 1995

\section{DISSERTATION}

Submitted in partial satisfaction of the requirements for the degree of

\section{DOCTOR OF PHILOSOPY}

In

Engineering/Applied Science

in the

\section{OFFICE OF GRADUATE STUDIES}

of the

UNIVERSITY OF CALIFORNIA

DAVIS

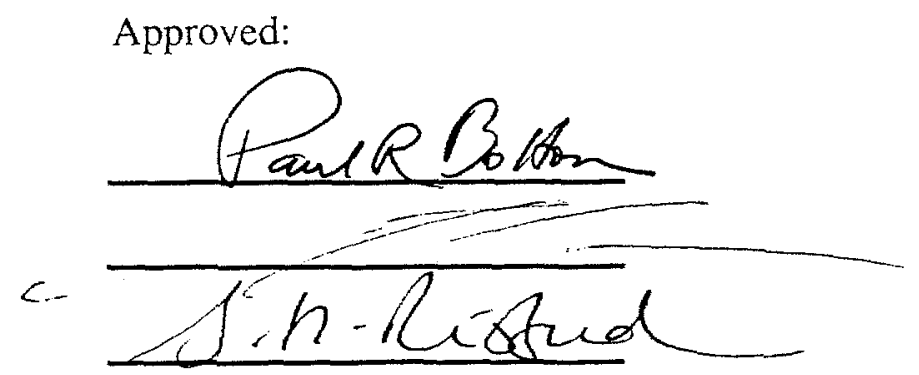

Committee in Charge

1999 
To my grandfather and my father,

Anson Burlingame Bullock (1911-1998) and Anthony Laird Bullock(1941-1986),

who taught me by example

that the first step in becoming

a good scientist is

becoming a good man

To my wonderful family and friends

Who loved and believed in me and my dreams even

When I had my doubts

To my nieces,

Katherine and Corinne Divine,

Who continually show me life is more than just lab work 


\section{ACKNOWLEDGEMENTS}

The completion of my research project, and indeed my training as a scientist, would not have been possible without the incredible assistance of the "cast of thousands" who helped me along the way. The following people all played an essential role in my graduate education, and their help is greatly appreciated.

My family, especially Mom, Max, Aunt Vivian, Tammy, Mark and the kids, all stood behind me and encouraged me, even in the days where I had lost hope. Dr. Iggy would have given up long ago without them.

My research advisor, Paul R. Bolton, undertook the Herculean task of teaching me the profession of science under extremely difficult circumstances. His courage, patience, determination, and humor continue to be an inspiration to me, and his friendship will not be forgotten.

My thesis advisor, Dr. Yin Yeh, was a great help along the way. His encouragement, assistance, and advice made a huge difference in my work over the last five years. Dr. Subash Risbud's reading of the manuscript is also greatly appreciated.

Many talented scientists and engineers played a helpful role in my research. Jim Hunter, Allen Hankla, and Bart Sellick took on the unenviable job of teaching me the gritty details of operating the Picosecond laser, and I thank them for the many hours they spent helping me. Jim Dunn, Frank Patterson, Bill White, and Peter Young all played an important role as teachers during my research, and I greatly appreciate their efforts. I also will not forget the help of Dwight Price, Jim Bonlie, John Foy, Al Ellis, and Judy Ticehurst. Their efforts on my behalf were above and beyond the call of duty.

Alexsey Komashko, Alexander Rubenchik, and Mike Feit all took me under their wing and opened my mind to the power and subtlety of plasma simulations, in general, and HYADES in particular. This work would not have been possible without their generous and patient assistance.

Outside the laboratory, many people made a huge difference in my research and my life during this challenging time. Gil, Dan, David, and especially Tony Megofna taught me a lot and made tough work of graduate school seem almost fun. They deserve much of the credit for keeping me going and turning the hard course work of grad school into more than just a series of tests. Outside LLNL, Iowa, Lynne, Jack, and Wenona all helped make life fun and helped take the sting out of the bumps and scraps of grad school.

Special thanks must go to Bill Chandler, Paul Springer, Mark Eckhart and Don Hoffmann, who supported me and the work both financially and morally.

And to all my friends and family who refuse to call me "doctor" unless there's an "Iggy" following it, this thesis is done, so pack up the camping gear, it's mountain time! 


\section{TABLE OF CONTENTS}

Title Page $\mathrm{i}$

Dedication ii

Acknowledgements................................................................ ii

Table of Contents.................................................................... iv

List of Figures..................................................................... vi

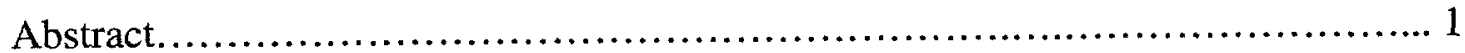

CHAPTER 1: A Primer on Laser-Induced Back Ablation.......................... 3

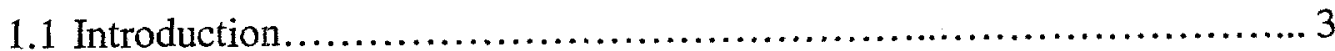

1.2 Conventional Laser-Induced Ablation of a Metal....................... 4

1.3 Long-Pulse Laser-Induced Back-Ablation........................... 8

1.4 Advantages of Short Laser Pulses in LIBA ............................ 11

CHAPTER 2: Experimental Method.............................................. 15

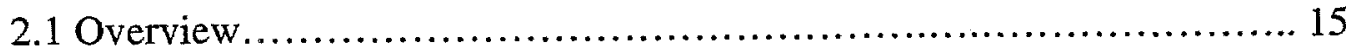

2.2 Two-Color Self-Referencing Interferometer.......................... 18

2.3 Time-Integrating Spectrometer.................................... 23

CHAPTER 3:Experimental Observations during LIBA.......................... 27

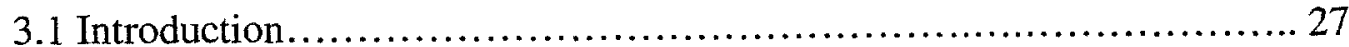

3.2 Laser-Induced Substrate Damage.................................... 28

3.3 Edge Velocity Measurements Using Shadowgraphs and Interferograms . 30

3.3.1 Edge Velocity................................................. 31

3.3.2 Edge Velocity Behavior at Low Laser Fluence.................. 39

3.3.3 Observations of Plume Edge Velocity at Multiple Delay Times 43 


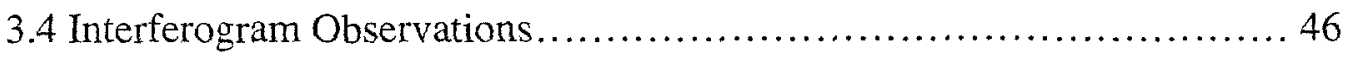

3.5 Spectral Observations.......................................... 55

3.6 Post-experimental Observations................................. 68

CHAPTER 4: Analysis and Discussion of LIBA Results...................... 78

4.1 Introduction.................................................... 78

4.2 Laser Fluence Transmission during Laser-Induced Substrate Damage.... 79

4.3 Laser Absorption in the Aluminum Thin Film....................... 86

4.4 HYADES Simulation of LIBA.................................... 88

4.5 Plume Temperature Analysis.................................. 102

4.6 Discussion of Practical Aspects of Short-Pulse LIBA................. 113

CHAPTER 5: Conclusions............................................... 122

5.1 Introduction..................................................... 122

5.2 LIBA Diagnostics........................................... 122

5.3 Useful aspects of Picoseond Pulse LIBA......................... 124

5.4 LIBA as a Laboratory Process................................. 125

5.5 Future Directions for LIBA Research........................... 126

APPENDIX A: A Ti:Sapphire-based CPA Picosecond Laser System.............. 128

A.1 Introduction......................................................... 128

A.2 Master Oscillator System.................................... 129

A.3. Chirped Pulse Amplification.................................... 134

A.4. Ti:Sapphire-Based Regenerative Amplifier........................ 138

A.5. Sequential Amplifier Chain..................................... 145

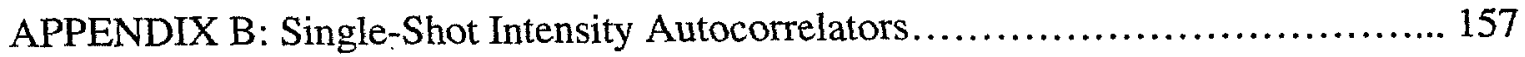




\section{LIST OF FIGURES}

1.1.1: $\quad$ Diagram of LIBA interaction scheme.............................. 3

1.2.1: Diagram of conventional laser ablation geometry ..................4

2.1.1: $\quad$ Diagram of LIBA interaction scheme............................15

2.1.2: Diagram of experimental apparatus used to direct picosecond pulses

into vacuum chamber...............................................................

2.2.1: Diagram of two-color self-referencing interferometer.................18

2.2.2: $\quad$ Diagram of plume interaction length, $\mathrm{T}$...........................21

2.3.1: Diagram of time-integrating spectrometer system..................23

2.3.2: Dependence of $\mathrm{HeNe}$ signal scattered from $\mathrm{Al}$ target on $\mathrm{Al}$ target

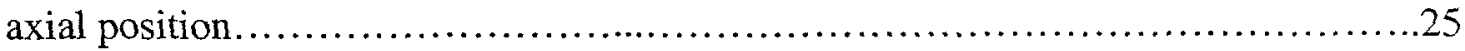

3.2.1: Oblique angle $C C D$ camera image of uncoated BK-7 substrate during

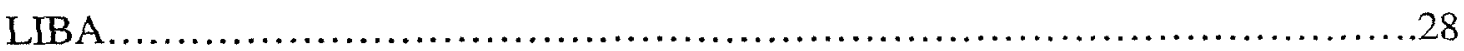

3.2.2: Dependence of the ratio of transmitted energy to incident laser energy on peak incident front surface fluence for $1.85 \mathrm{ps}$ laser pulses. .29

3.2.3: Dependence of the ratio of transmitted energy to incident laser energy on peak incident front surface fluence for $3.33 \mathrm{ps}$ laser pulses................30

3.3.1.1: $\quad$ Interferograms and shadowgraphs showing LIBA-generated plumes produced by 3.33 ps pulses ablating $.50 \mu \mathrm{m}$ films...............................32

3.3.1.2: Interferograms and shadowgraphs showing LIBA-generated plumes produced by $1.85 \mathrm{ps}$ pulses ablating $.50 \mu \mathrm{m}$ films...............................32

3.3.1.3: $\quad$ Interferograms and shadowgraphs showing LIBA-generated 
plumes produced by 3.33 ps pulses ablating $.25 \mu \mathrm{m}$ films

3.3.1.4: Interferograms and shadowgraphs showing LIBA-generated

plumes produced by $1.85 \mathrm{ps}$ pulses ablating $.25 \mu \mathrm{m}$ films..

3.3.1.5: Diagram of mapping process for comparison of edge velocity

to beam profile.

3.3.1.6: Plot of edge velocity dependence on front surface fluence for $.25 \mu \mathrm{m}$ film ablated by $1.85 \mathrm{ps}$ pulses.

3.3.1.7: Plot of edge velocity dependence on front surface fluence for $.25 \mu \mathrm{m}$ film ablated by 3.33 ps pulses.

3.3.1.8: $\quad$ Plot of edge velocity dependence on front surface fluence for $.50 \mu \mathrm{m}$ film ablated by $1.85 \mathrm{ps}$ pulses.

3.3.1.9: $\quad$ Plot of edge velocity dependence on front surface fluence

for $.50 \mu \mathrm{m}$ film ablated by 3.33 ps pulses.

3.3.2.1: $\quad$ Plot of edge velocity as a function of front surface fluence

for plumes produced by $0.25 \mu \mathrm{m}$ films ablated by 3.33 ps laser pulses.

3.3.2.2: $\quad$ Plot of edge velocity as a function of front surface fluence

for plumes produced by $0.25 \mu \mathrm{m}$ films ablated by 1.85 ps laser pulses.

3.3.2.3.Plot of edge velocity as a function of front surface fluence

for plume produced by $0.50 \mu \mathrm{m}$ films ablated by $3.33 \mathrm{ps}$ laser pulses.

3.3.3.1: $\quad$ Images of a metal plume formed by a $0.25 \mu \mathrm{m}$ thin film ablated

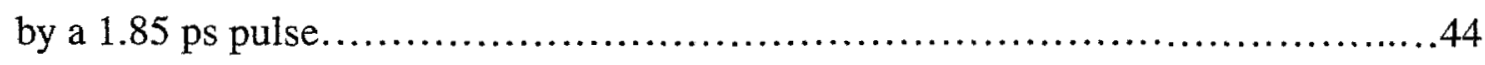

3.3.3.2: $\quad$ Plot of edge velocity for a $.25 \mu \mathrm{m}$ thin film ablated by a

1.85 ps laser pulse. 
3.3.3.3. Plot of edge velocity data set for a $.25 \mu \mathrm{m}$ thin film ablated by a 1.85 ps laser pulse...

3.4.1: $\quad$ An interferogram $(527 \mathrm{~nm})$ of a $.25 \mu \mathrm{m}$ film ablated by a 3.33 ps

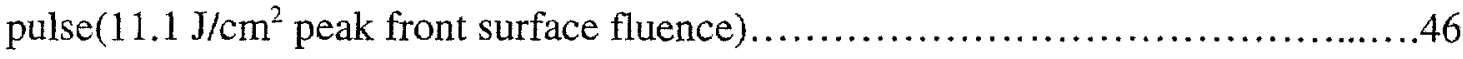

3.4.2: $\quad$ Interferograms of a $0.1 \mu \mathrm{m} \mathrm{Al} \mathrm{film} \mathrm{ablated} \mathrm{by} 3.33$ ps ablation

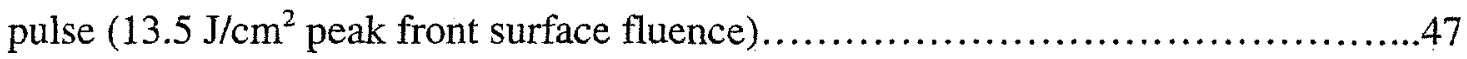

3.4.3: Diagram describing how a 2-D spatial laser intensity profile is transformed into an approximate plume shape................................48

3.4.4(A): $\quad$ Plot of neutral density on the centerline of a $0.1 \mu \mathrm{m} \mathrm{Al} \mathrm{film}$ ablated by 3.33 ps ablation pulse $\left(13.5 \mathrm{~J} / \mathrm{cm}^{2}\right.$ peak front surface fluence).............50 3.4.4(B): $\quad$ Plot of electron density on the centerline of an Al plume generated by a $0.1 \mu \mathrm{m} \mathrm{Al}$ film ablated by 3.33 ps ablation pulse

$\left(13.5 \mathrm{~J} / \mathrm{cm}^{2}\right.$ peak front surface fluence) .50

3.4.3(C): Plot of the ratio of electron density and neutral density on the centerline of an Al plume generated by a $0.1 \mu \mathrm{m}$ Al film ablated by $3.33 \mathrm{ps}$ ablation pulse $\left(13.5 \mathrm{~J} / \mathrm{cm}^{2}\right.$ peak front surface fluence).

3.4.5(A): $\quad$ A $527 \mathrm{~nm}$ Interferogram of $0.1 \mu \mathrm{m} \mathrm{Al} \mathrm{film} \mathrm{ablated} \mathrm{by} 3.33$ ps pulses at a peak front surface fluence of $1.77 \mathrm{~J} / \mathrm{cm}^{2}$ .51

3.4.5(B): A $1053 \mathrm{~nm}$ interferograms of $0.1 \mu \mathrm{m}$ Al film ablated by

3.33 ps pulses at a peak front surface fluence of $1.77 \mathrm{~J} / \mathrm{cm}^{2}$

3.4.6(A): $\quad$ Plot of neutral density for a $0.1 \mu \mathrm{m} \mathrm{Al} \mathrm{film} \mathrm{ablated} \mathrm{by}$

3.33 ps ablation pulse $\left(1.77 \mathrm{~J} / \mathrm{cm}^{2}\right.$ peak front surface fluence) $\ldots \ldots \ldots \ldots \ldots \ldots \ldots . .52$

3.4.6(B): $\quad$ Plot of electron density for a $0.1 \mu \mathrm{m} \mathrm{Al} \mathrm{film} \mathrm{ablated} \mathrm{by}$ 
3.33 ps ablation pulse $\left(1.77 \mathrm{~J} / \mathrm{cm}^{2}\right.$ peak front surface fluence) $\ldots \ldots \ldots \ldots \ldots \ldots \ldots . \ldots 2$

3.4.7: Sketch of 1-D semi-infinite heat transport problem with time

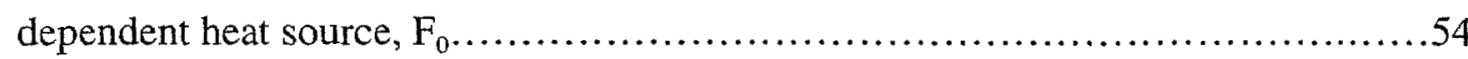

3.4.8: $\quad$ Plot of 1-D temperature distribution of semi-infinite Al slab after

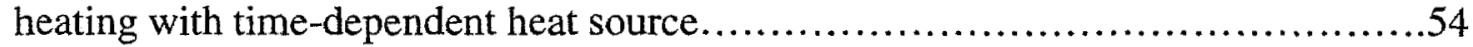

3.5.1: $\quad$ Diagram of "clipped" (A) and "unclipped" (B) target geometry.......55

3.5.2: $\quad$ Plot of front surface substrate damage spectra caused by a 3.33ps

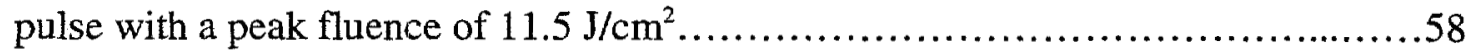

3.5.3: Plot of front surface substrate damage spectra caused by a $3.33 \mathrm{ps}$

pulse with a peak fluence of $31.1 \mathrm{~J} / \mathrm{cm}^{2}$

3.5.4: $\quad$ Spectra originating from an $\mathrm{Al}$ plume produced by a $0.25 \mu \mathrm{m}$

Al film ablated by a $1.85 \mathrm{ps}$ pulse $\left(19.0 \mathrm{~J} / \mathrm{cm}^{2}\right.$ peak fluence $) . \ldots \ldots \ldots \ldots \ldots \ldots \ldots \ldots$

3.5.5: Calculated continuum emission for a black body at $\mathrm{T}=0.25 \mathrm{eV} \ldots \ldots .61$

3.5.6: $\quad$ Spectra originating from an $\mathrm{Al}$ plume produced by a $0.50 \mu \mathrm{m}$

Al film ablated by a $3.33 \mathrm{ps}$ pulse $\left(12.3 \mathrm{~J} / \mathrm{cm}^{2}\right.$ peak fluence $) \ldots \ldots \ldots \ldots \ldots \ldots \ldots . \ldots 62$

3.5.7: $\quad$ Spectra originating from an Al plume produced by a $0.25 \mu \mathrm{m}$

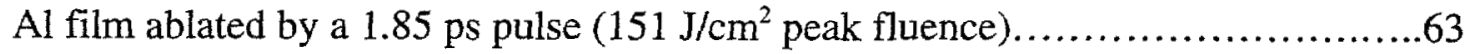

3.5.8: $\quad$ Spectra originating from an $\mathrm{Al}$ plume produced by a $0.50 \mu \mathrm{m}$

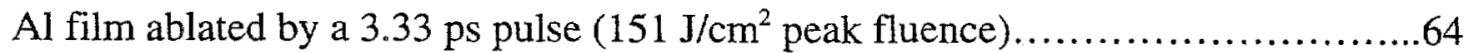

3.5.9: $\quad$ Spectra originating from an Al plume produced by a $0.25 \mu \mathrm{m}$

Al film ablated by a $3.33 \mathrm{ps}$ pulse $\left(12.3 \mathrm{~J} / \mathrm{cm}^{2}\right.$ peak fluence $) . . \ldots \ldots \ldots \ldots \ldots \ldots . . \ldots 6$

3.5.10: $\quad$ Spectra originating from an $\mathrm{Al}$ plume produced by a $0.25 \mu \mathrm{m} \mathrm{Al} \mathrm{film}$

ablated by a $3.33 \mathrm{ps}$ pulse $\left(25.7 \mathrm{~J} / \mathrm{cm}^{2}\right.$ peak fluence)..........................67 
3.5.11: $\quad$ Plot of the line ratio of the observed $655.5 \mathrm{~nm} \mathrm{Si}_{1}$ and the 624

$\mathrm{Al}$ II line as a function of front surface fluence for the $\mathrm{Al}$ plume produced by

a $0.25 \mu \mathrm{m}$ Al film ablated by a 3.33 ps pulse..................................68

3.6.1: $\quad$ Picture of $0.25 \mu \mathrm{m} \mathrm{Al}$ target after LIBA........................69

3.6.2: $\quad$ Plot of transverse hole size as a function of peak front surface

fluence for ablations holes produced in $0.25 \mu \mathrm{m}$ films by 3.33 ps pulses..............70

3.6.3: $\quad$ Plot of transverse hole size as a function of peak front surface

fluence for ablations holes produced in $0.25 \mu \mathrm{m}$ films by $1.85 \mathrm{ps}$ pulses..............71

3.6.4: $\quad$ Plot of transverse hole size as a function of peak front surface

fluence for ablations holes produced in $0.50 \mu \mathrm{m}$ films by 3.33 ps pulses.............72

3.6.5: Plot of transverse height distribution for the case of $0.50 \mu \mathrm{m}$

films ablated by 3.33 ps pulses....................................... 73

3.6.6: $\quad$ Photograph of the $0.50 \mu \mathrm{m} \mathrm{Al} \mathrm{target} \mathrm{after} \mathrm{ablation} \mathrm{by} 3.33 \mathrm{ps}$

laser pulses. The white bar above the target is a scale marker and is $1575 \mu \mathrm{m}$ in

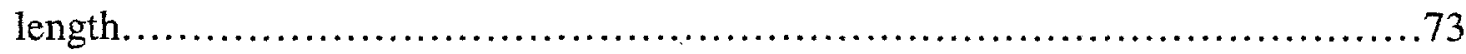

3.6.7: Interferogram of receiving substrate........................... 75

4.2.1: $\quad$ Diagram of laser interaction with aluminum target..................79

4.2.2: Diagram showing transmitted fluence as the leading edge of the

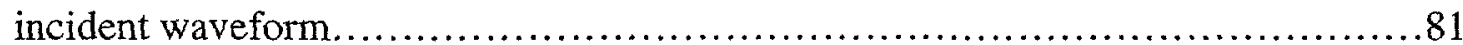

4.2.3: A plot of Al plume edge velocity as a function of incident front-surface fluence for $0.25 \mu \mathrm{m}$ films ablated by 1.85 psec laser pulses is shown..............83 
4.2.4: A plot of $\mathrm{Al}$ plume edge velocity as a function of incident front-surface fluence for $0.25 \mu \mathrm{m}$ films ablated by $3.33 \mathrm{psec}$ laser pulses is shown..............84

4.2.5: A plot of Al plume edge velocity as a function of incident front-surface fluence for $0.50 \mu \mathrm{m}$ films ablated by $3.33 \mathrm{psec}$ laser pulses is shown...84 4.2.6: A plot of Al plume edge velocity as a function of incident front-surface fluence for $0.50 \mu \mathrm{m}$ films ablated by 1.85 psec laser pulses is shown....85 4.2.7: Graph of $t_{0}$ as a function of front surface fluence $\left(\operatorname{Sech}^{2}(t)\right.$ temporal

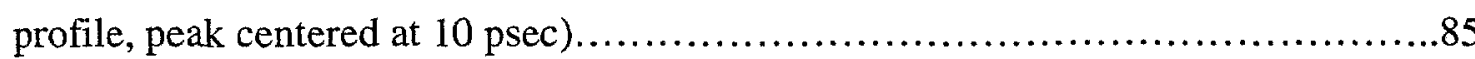

4.3.1: $\quad$ Plasma profiles showing the initial and expanding density profiles.

The Helmholtz equation is solved using the "expanding plasma" density profile.......87

4.3.2: $\quad$ Plot of reflectivity and absorption as a function of scale length.

The scale length axis is in units of wavelength $(1053 \mathrm{~nm})$ .87

4.4.1: $\quad$ Plot of calculated velocity of the first zone of a $0.5 \mu \mathrm{m} \mathrm{Al} \mathrm{film}$ $2 \mathrm{~ns}$ after interaction with a 3.33 ps ablation pulse (peak front surface fluence

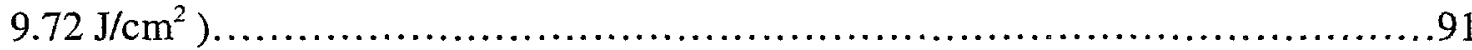

4.4.2: $\quad$ Plot of observed edge velocity and calculated edge velocity as a function of front surface fluence for a $0.5 \mu \mathrm{m} \mathrm{Al} \mathrm{film} \mathrm{ablated} \mathrm{by} \mathrm{a} 3.33$ ps pulse......91 4.4.3: $\quad$ Plot of observed edge velocity and calculated edge velocity as a function of front surface fluence for a $0.25 \mu \mathrm{m} \mathrm{Al}$ film ablated by a 3.33 ps pulse.

4.4.4: $\quad$ Plot of observed edge velocity and calculated edge velocity as a function of front surface fluence for a $0.25 \mu \mathrm{m} \mathrm{Al} \mathrm{film} \mathrm{ablated} \mathrm{by} \mathrm{a} 1.85$ ps pulse....93 4.4.5: $\quad$ Plot of observed edge velocity and calculated edge velocity 
as a function of front surface fluence for a $0.50 \mu \mathrm{m} \mathrm{Al}$ film ablated by a

1.85 ps pulse.

4.4.6: $\quad$ Plot of HYADES-calculated density, $\rho\left(\mathrm{g} / \mathrm{cm}^{3}\right)$, of interface zone (zone 50) as a function of time for plumes generated by $0.25 \mu \mathrm{m}$ Al films ablated by 3.33 ps pulses.

4.4.7: Plot of HYADES-calculated density, $\rho\left(\mathrm{g} / \mathrm{cm}^{3}\right)$, of interface zone (zone 50) as a function of time for plumes generated by $0.5 \mu \mathrm{m} \mathrm{Al}$ films ablated by 3.33 ps pulses

4.4.8: $\quad$ Plot of HYADES-calculated density as a function of time for plumes generated by $0.25 \mu \mathrm{m} \mathrm{Al}$ films ablated by 1.85 ps pulses. .97

4.4.9: $\quad$ Plot of HYADES-calculated pressure as a function of time for plumes generated by $0.25 \mu \mathrm{m}$ Al films ablated by 3.33 ps pulses.

4.4.10: $\quad$ Plot of interface pressure as a function of time. Plot (A) corresponds a plume generated by ablating a $0.25 \mu \mathrm{m} \mathrm{Al} \mathrm{film} \mathrm{with} \mathrm{a} 1.85$ ps pulse $\left(0.918 \mathrm{~J} / \mathrm{cm}^{2}\right.$ peak front surface fluence) (ablation threshold fluence) 101 4.5.1: $\quad$ Plot of time-integrated spectra from Al plume $(0.25 \mu \mathrm{m}$ film, a 3.33 ps pulse, $12.3 \mathrm{~J} / \mathrm{cm}^{2}$ peak front surface fluence) 103

4.5.2: $\quad$ Plot of calculated temperature based on relative line ratios $470 \mathrm{~nm} / 559 \mathrm{~nm}$ and $470 \mathrm{~nm} / 624 \mathrm{~nm}$ for plumes generated by $0.25 \mu \mathrm{m}$ Al films ablated by 3.33 ps pulses. 107

4.5.3: $\quad$ Plot of calculated temperature based on relative line ratios $470 \mathrm{~nm} / 559 \mathrm{~nm}$ and $470 \mathrm{~nm} / 624 \mathrm{~nm}$ for plumes generated by $0.5 \mu \mathrm{m}$ Al films ablated by 1.85 ps pulses. 108 
4.5.4: Plot of calculated temperature based on relative line ratios

$470 \mathrm{~nm} / 559 \mathrm{~nm}$ and $470 \mathrm{~nm} / 624 \mathrm{~nm}$ for plumes generated by $0.1 \mu \mathrm{m}$ Al films

ablated by 3.33 ps pulses............................................... 108

4.5.5: HYADES calculations of interface zone temperature as a function

of time for plumes generated by $0.25 \mu \mathrm{m}$ films ablated by 3.33 ps pulses..............110

4.5.6: HYADES calculations of interface zone temperature as a function

of time for plumes generated by $0.5 \mu \mathrm{m}$ films ablated by 3.33 ps pulses..............111

4.5.7: $\quad$ Plot of HYADES-calculated temperature, $\mathrm{T}$, as a function of

time for plumes generated by $0.25 \mu \mathrm{m} \mathrm{Al}$ films ablated by 1.85 ps pulses...........112

4.6.1: $\quad$ Interferograms of $0.5 \mu \mathrm{m} \mathrm{Al} \mathrm{film} \mathrm{ablated} \mathrm{with} 3.33$ ps pulses.........114

4.6.2: $\quad$ Interferograms $(527 \mathrm{~nm})$ of two Al plumes, generated with

$0.25 \mu \mathrm{m}$ Al film ablated with either a 1.85 ps laser pulse (A) or a 3.33 ps

laser pulse (B) (peak front surface fluence $(A)=21.5 \mathrm{~J} / \mathrm{cm}^{2},(B)=19.0 \mathrm{~J} / \mathrm{cm}^{2}$ ) $\ldots \ldots \ldots .114$

4.6.3: Oblique view CCD time-integrated images of in-flight plume and

substrate damage emission for $0.25 \mu \mathrm{m}$ films ablated by 3.33 ps pulses.............116

4.6.4: $\quad$ Shadowgraph images of in-flight plume generated by $0.25 \mu \mathrm{m}$

films ablated by 3.33 ps pulses........................................... 116

4.6.5: Oblique view CCD time-integrated images of in-flight plume and substrate damage emission for $0.25 \mu \mathrm{m}$ films ablated by 1.85 ps pulses.............117

4.6.6: $\quad$ Plot of observed spectra for $0.25 \mu \mathrm{m}$ film ablated by 3.33 ps pulse

$\left((A)=11.1 \mathrm{~J} / \mathrm{cm}^{2}\right.$ peak front surface fluence and $(B)=12.3 \mathrm{~J} / \mathrm{cm}^{2}$ peak front surface

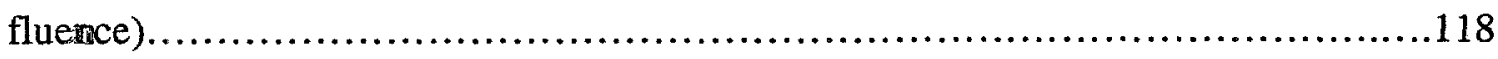


4.6.7: $\quad$ An interferogram $(527 \mathrm{~nm})$ of a $.25 \mu \mathrm{m}$ film ablated by a $3.33 \mathrm{ps}$

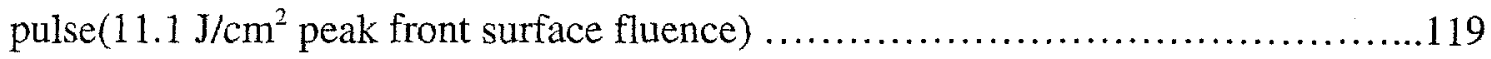

A.2.1: Diagram of a Spectra-Physics Ti:Sapphire-based Tsunami

Oscillator.................................................................... 130

A.2.2: $\quad$ Energy Band Structure for Ti:Sapphire............................ 131

A.2.3: $\quad$ Absorption and Emission Spectra for Ti:Sapphire...................132

A.2.4: $\quad$ Diagram of 4-prism GVD compensator..........................134

A.3.1: $\quad$ Diagram of pulse stretcher system...............................136

A.3.2: $\quad$ Diagram of vacuum compressor system..........................137

A.4.1: Diagram of Ti:Sapphire-Based Regenerative Amplifier. The

oscillator beam path, shown as a solid line, describes the cavity beam path..........139

A.4.2: $\quad$ Unseeded Regenerative Amplifier Leakage Signal. This signal

originates from leakage through a cavity end mirror which is directed into a

photodiode................................................................ 140

A.4.3: $\quad$ Seeded Regenerative Amplifier Leakage Signal.....................143

A.5.1: $\quad$ Diagram of sequential amplifier chain............................ 146

A.5.2: $\quad$ Diagram of faraday rotator....................................... 153

B.1: Diagram of single-shot SHG-based autocorrelator.................158

Tables

3.3.1.1: $\quad$ Table of threshold fluence values as a function of film thickness and

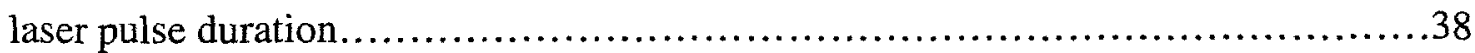

2.5.1: A list of the Si I, Si II, and Si III spectral lines in the range lines of

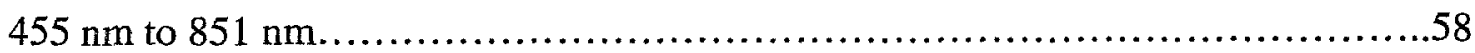


3.5.2: $\quad$ Table of observed spectral lines in spectrum presented in

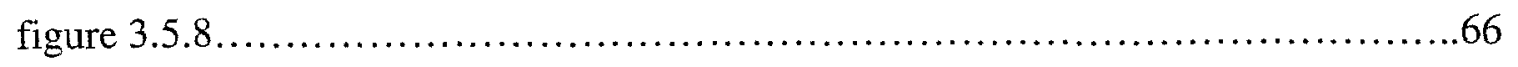

3.6.1. Table of ablation fluence threshold estimates based on low-fluence edge

velocity data and ablation hole size measurements.................................71 


\begin{abstract}
Experiments were performed to understand laser-induced back-ablation of Al film targets with picosecond laser pulses. Al films deposited on the back surface of BK-7 substrates are ablated by picosecond laser pulses propagating into the Al film through the substrate. The ablated $\mathrm{Al}$ plume is transversely probed by a time-delayed, two-color subpicoseond (500 fs) pulse, and this probe is then used to produce self-referencing interferograms and shadowgraphs of the $\mathrm{Al}$ plume in flight. Optical emission from the $\mathrm{Al}$ target due to LIBA is directed into a time-integrated grating spectrometer, and a timeintegrating $\mathrm{CCD}$ camera records images of the $\mathrm{Al}$ plume emission. Ablated $\mathrm{Al}$ plumes are also redeposited on to receiving substrates. A post-experimental study of the $\mathrm{Al}$ target and recollected deposit characteristics was also done using optical microscopy, interferometry, and profilometry.

In this high laser intensity regime, laser-induced substrate ionization and damage strongly limits transmitted laser fluence through the substrate above a threshold fluence. The threshold fluence for this ionization-based transmission limit in the substrate is dependent on the duration of the incident pulse. The substrate ionization can be used as a dynamic control of both transmitted spatial pulse profile and ablated Al plume shape. The efficiency of laser energy transfer between the laser pulse incident on the Al film and the ablated A1 plume is estimated to be of order 5\% and is a weak function of laser pulsewidth. The $\mathrm{Al}$ plume is highly directed. Low plume divergence $\left(\theta_{\text {divergence }}<5^{\circ}\right)$ shows the ablated plume temperature to be very low at long time delays ( $\mathrm{T}<<0.5 \mathrm{eV}$ at delays of $255 \mathrm{~ns}$ ). Spectroscopic observations and calculations indicate that, in early time $(\mathrm{t}<100 \mathrm{ps}$ ), the $\mathrm{Al}$ film region near the substrate/metal interface is at temperatures of
\end{abstract}


order $0.5 \mathrm{eV}$. Interferograms of $\mathrm{Al}$ plumes produced with $0.1 \mu \mathrm{m}$ films show these plumes to be of high neutral atom density $\left(n_{n}\right.$ of order $\left.10^{18} \mathrm{~cm}^{-3}\right)$ and weakly ionized $\left(n_{\mathrm{e}} / \mathrm{n}_{\mathrm{n}}<0.001\right)$ at long time delays of order $200 \mathrm{~ns}$. Recollections of Al plumes confirm the low plume divergence and demonstrate high material adhesion to the receiving substrate, as well as a higher $\mathrm{Al}$ material yield than that of conventional pulsed laser deposition. Al redepositions are also highly conductive at the deposit/substrate interface, suggesting possible applications in selective laser-assisted bonding. 


\section{CHAPTER 1. A PRIMER ON LASER-INDUCED BACK-ABLATION}

\subsection{Introduction}

Conventional laser ablation, in which a laser pulse strikes a solid and explosively removes material from a front surface, is a well-known physical process and forms the basis of important material science techniques such as PLD $^{1}$. By contrast, Laser-Induced Back-Ablation (LIBA) involves propagating a laser pulse through a transparent substrate and into a metal thin film on the rear surface (see Figure 1.1.1). The laser pulse deposits energy into the thin film at the metal/substrate interface, and this energy ablates the entire thin film ${ }^{2}$. LIBA involves different ablation dynamics than that of conventional ablation and can result in metal vapor plumes with interesting and potentially useful characteristics $^{3}$.

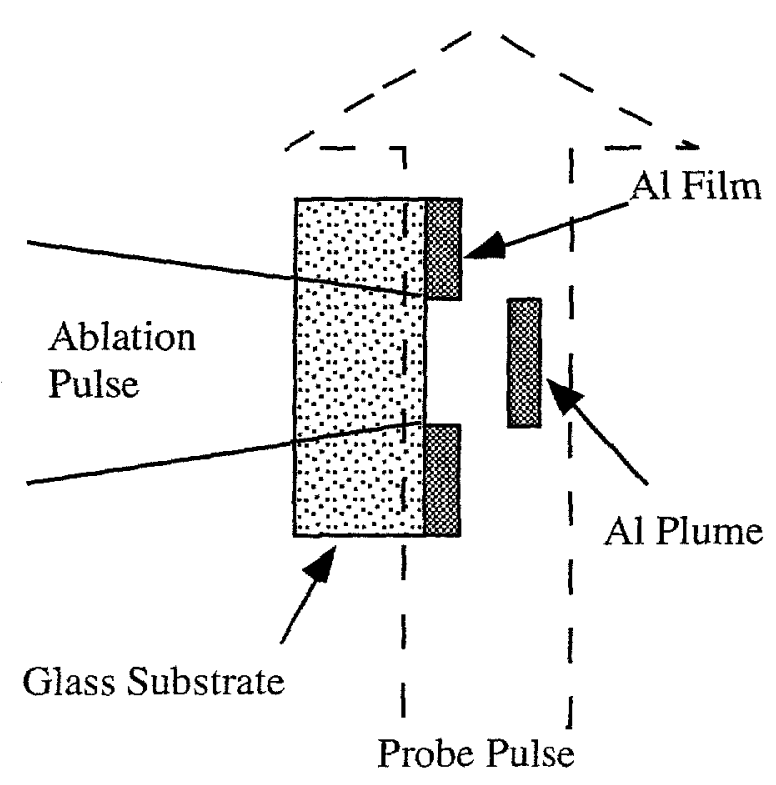

Figure 1.1.1. Diagram of LIBA interaction scheme.

I begin this primer by looking at conventional front surface ablation. I look at the laser/matter interaction and the subsequent ablation of material from the front surface. I then will compare long pulse LIBA with conventional ablation. I will also discuss new 
physical features important to understanding LIBA. Finally, I will discuss short-pulse LIBA, and I will show both the similarities and differences between short and long pulse LIBA.

\subsection{Conventional Laser-Induced Ablation of a Metal}

In conventional laser ablation, a high-energy laser pulse is focused into a metal surface.

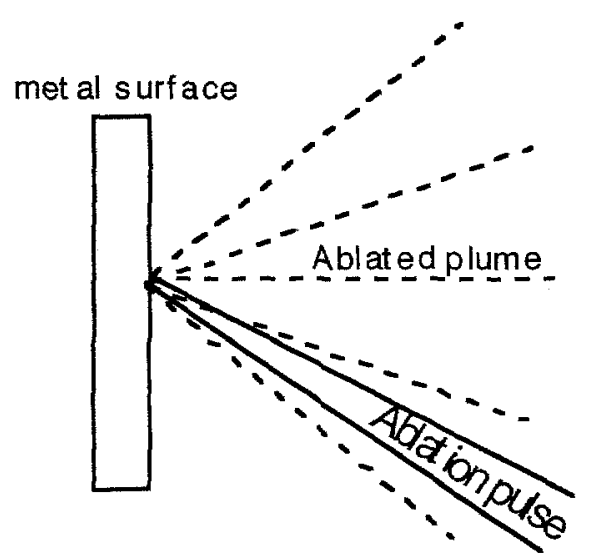

Figure 1.2.1. Diagram of conventional laser ablation geometry.

On arrival, the leading edge of the laser pulse interacts with the conduction electrons in the metal surface. The conduction electron density $\left(\mathrm{n}_{\mathrm{e}} \approx 1.8 \times 10^{23} \mathrm{~cm}^{-3}\right)$ is above the critical electron density $\left(\mathrm{n}_{\mathrm{e}} \approx 10^{21} \mathrm{~cm}^{-3}\right.$ at $\left.1053 \mathrm{~nm}\right)$, and most of the incident laser pulse energy is reflected away from the thin film. The small portion not reflected is absorbed within a few skin depths $(16.8 \mathrm{~nm}$ at $1053 \mathrm{~nm})$. The interaction of the laser pulse with the conduction electrons also produces a reflected wave. The reflectivity of this metal surface at normal incidence is described by the expression below ${ }^{4}$.

$$
R=\frac{[1-n]^{2}+\frac{\sigma}{\omega \varepsilon_{0}}}{[1+n]^{2}+\frac{\sigma}{\omega \varepsilon_{0}}}
$$


Where $\mathrm{n}$ is the real component of the index of refraction of the metal, $\sigma$ is the real AC conductivity of the metal, $\omega$ is the angular frequency of the laser pulse, and $\varepsilon_{0}$ is the permittivity of free space.

The reflectivity of the metal surface at $1053 \mathrm{~nm}$ prior to laser heating and ablation is $95 \%$, and the $95 \%$ of the incident energy of the leading edge is reflected away from the surface. The remaining $5 \%$ is absorbed by electron-phonon collisions ${ }^{5}$ in the metal inside the region a few skin depths (see figure 1.2.1). The effective thermalization time between the electrons and atoms in the solid, $\tau_{\mathrm{e}-\mathrm{T}}$, is less than $1 \mathrm{ps}^{6}$, and the energy distribution can be considered to be thermalized at times a few picoseonds after the ablation pulse. On this timescale, the metal structure and reflectivity will then change during the laser interaction. Generally, conductivity, and therefore reflectivity, will drop as temperature rises, and therefore the metal begins to absorb marginally more of the incident pulse as temperatures rise to near melting temperature.

As temperatures increase above the $\mathrm{Al}$ melting temperature, $\mathrm{T}_{\text {melt }}(933.5 \mathrm{~K})^{7}$, the aluminum material can begin to leave the metal surface. Two primary mechanisms dominate the conventional ablation process. The first is thermal sputtering where (after sufficient heating) $\mathrm{Al}$ atoms evaporate off the surface ${ }^{8}$. This process requires temperatures $\mathrm{T}>\mathrm{T}_{\text {melt }}$ although temperatures cannot exceed the thermodynamic critical temperature, $\mathrm{T}_{\mathrm{cr}}$. Following the argument by Kelly ${ }^{9}$, the asymptotic depth of material removed by the laser pulse ("top-hat" temporal profile) can be described by the expression below.

$$
\text { depth } \approx\left(p_{a t m} T^{\frac{1}{2}} \tau / M^{\frac{1}{2}} \Delta H_{v}\right) \times 1.53 \times 10^{6} \mathrm{~nm} / \mathrm{pulse}
$$

where $T$ is the maximum surface temperature (in $\mathrm{eV}$ ), $\mathrm{p}_{\text {atm }}$ is the atmospheric pressure, $\tau$ is the laser pulse duration, $\mathrm{M}$ is the atomic weight of aluminum, and $\Delta \mathrm{H}_{\mathrm{v}}$ is the heat of vaporization (in $\mathrm{eV}$ ) 
In this case, liberating $1 \mathrm{nn}$ of $\mathrm{Al}$ from a solid with one $12 \mathrm{~ns}$ laser pulse requires a peak Al temperature of $3500 \mathrm{~K}$. The material which leaves the surface by this mechanism is a vapor phase $\mathrm{Al}$ plume. The second dominant ablation mechanism is hydrodynamic sputtering. This process involves the formation of liquid droplets which are ejected from the surface due to transient heating ${ }^{8}$. Based on the thermal expansion model offered by Kelly and Rothenberg ${ }^{9}$, the surface undergoes melting and droplets form on the front surface. The bulk solid away from the surface is not heated directly by the laser, however, heat diffuses into the bulk, cooling the surface. Contraction due to cooling accelerates the surface towards the bulk material and away from the droplets, ejecting them from the front surface. This mechanism leads to liquid metal being ejected into vacuum. With these two mechanisms, several other, secondary mechanisms, such as effusion and outflow, operate in concert, and the combination of these mechanisms operating together allow the ablated material to be described by the laws of gas-dymanics ${ }^{8}$.

$$
\begin{aligned}
& \frac{\partial a}{\partial t}+u \frac{\partial a}{\partial x}+\frac{(\gamma-1) a}{2} \frac{\partial u}{\partial x}=0 \\
& \frac{\partial u}{\partial t}+u \frac{\partial u}{\partial x}+\frac{2 a}{\gamma-1} \frac{\partial a}{\partial x}=0
\end{aligned}
$$

Where $a=\left(\gamma k_{b} T / m\right)^{1 / 2}$ is the sound speed, $u$ is the flow velocity, and $\gamma$ is the heat-capacity ratio.

As ablation begins to occur, the ejected particles can continue to interact with the incident laser pulse. The incoming laser pulse can heat the ejected particles and also 
ionize the atoms by predominantly collisional ionization. In early time, the ejected particles are largely neutrally-charged, and the laser-driven heating is dominated by inverse-bremsstrahlung absorption of the laser light in a collision between the electrons and the neutral atoms. This absorption coefficient is described by the expression below ${ }^{10}$.

$$
\begin{aligned}
& \alpha_{N}=C_{0}\left(e^{\frac{h v}{k T}}-1\right) h v N_{e} \sum_{n=1}^{n^{*}} g_{n n}(h v, T) N(n) \\
& C_{0}=\frac{4}{3 \sqrt{2}} \frac{\hbar^{2} c^{2} \alpha^{3}}{(\pi m k T)^{\frac{3}{2}}} \frac{1}{v^{3}} \quad(1.4)
\end{aligned}
$$

where $v$ is the frequency of the laser, $T$ is the electron temperature, $N_{e}$ is the electron density, $N(n)$ is the number density of atoms at the excited state $n, \alpha$ is the fine structure constant, $\mathrm{m}$ is the electron mass, and $\mathrm{g}_{\mathrm{n}}$ is related to collision cross-sections of electrons with neutral atoms in the nth excited state.

The ejected atoms are collisionally ionized by free electrons accelerated by the incident laser field. At laser intensities of $1 \mathrm{GW} / \mathrm{cm}^{2}$, the growth rate in free electrons can be described by $\mathrm{dN} / \mathrm{dt}=\alpha_{\mathrm{e}} \mathrm{N}_{\mathrm{a}} \mathrm{N}_{\mathrm{e}}$, where $\mathrm{N}_{\mathrm{a}}$ and $\mathrm{N}_{\mathrm{e}}$ are the atom and electron number densities $\left(\mathrm{N}_{\mathrm{e}}<<\mathrm{N}_{\mathrm{a}}\right)$ and $\alpha_{\mathrm{e}}$ is the avalanche ionization coefficient ${ }^{11}$. This last coefficient is for ionization from the ground-state. As electron and ionic densities rise to levels near $10^{19}$ $\mathrm{cm}^{-3}$, electron-ion inverse-bremsstrahlung begins to dominate the absorption process, and this absorption coefficient is described below.

$$
\alpha=\left(3.69 \times 10^{8}\right) \frac{Z^{3} N_{i}^{2}}{T^{\frac{1}{2}} v^{3}}\left(1-e^{-h v / k T}\right)
$$

where $\mathrm{N}_{\mathrm{i}}$ is the ionic density and $\mathrm{Z}$ is average ionic charge state.

Here, $\alpha$ is in units of $\mathrm{cm}^{-1}$. This absorption continues to heat and ionize the plume, thereby limiting the energy delivered to the metal surface. 
Conventional laser ablation, specifically for laser pulses of order $10 \mathrm{~ns}$ in duration, involves the ejection and subsequent heating and ionization of metal atoms and droplets. The interaction geometry causes long laser pulses to heat up ejected material and thereby lose intensity prior to striking the ablating metal surface. Conventionally ablated plumes can have temperatures of order several $\mathrm{eV}^{12}$, and due to this temperature, are highly divergent.

This laser/plume interaction causes heating and ionization, which prevents the probing of plume material near the metal surface. Experimental study of the plume during the laser/matter interaction is limited.

\subsection{Long-Pulse Laser-Induced Back-Ablation}

In contrast to conventional ablation, the LIBA process involves the laser placing energy initially at the metal/substrate interface(see figure 1.1.1). In early time when the Al film is a solid, the Al layer near the interface exhibits the same optical behavior as a metal surface under laser heating with metal temperatures $\mathrm{T}_{\text {metal }}<\mathrm{T}_{\text {melt }}$. However, as Al film temperatures exceed $\mathrm{T}_{\text {melt }}$, the $\mathrm{Al}$ in the region is restrained by the outer Al layers. As temperatures reach the regime in which thermal and hydrodynamic sputtering is possible under conventional ablation, the LIBA geometry restricts the $\mathrm{Al}$ interface region from expansion, because the outer Al layers further away from the interface resist the expansion. As a result, the Al layer continues to absorb laser energy. This heat diffuses into the surrounding film, but for high fluence pulses, the rate of energy deposition is far higher than that of diffusion. This causes the interface layer to increase in temperature and pressure until the increased pressure separates the film from the substrate ${ }^{13}$, thereby causing back-ablation of the entire film. 
Prior to expansion and ablation of the $\mathrm{Al}$ film, the laser/metal interaction at the interface behaves much like the laser/metal interaction at a metal surface under conventional ablation. The absorption of the incident laser pulse energy changes after ablation begins, however, since the entire film leaves the surface. As an ablated plume with neutral atom density far less than solid density, the Al plume does not have a large number of conduction electrons. Therefore $\mathrm{Al}$ reflectivity drops as the thin film expands to a density below solid density. Furthermore, the ablated plume is unlikely to have a planar electron profile suitable for high on-axis reflectance. Modeling of the timeintegrated on-axis reflectance of gaussian pulses by Al targets under long-pulse backablation shows that the reflectivity of the Al film can be assumed to go to zero after ablation $^{14}$. In cases for which ablation occurs on a timescale comparable to the pulse duration, the trailing edge of the pulse interacts with the ablated Al plume. The absorption mechanism is similar to that observed in conventional ablation, although the extreme heating required to build back-ablation pressure may increase the temperature and ionization state of the $\mathrm{Al}$ in the interface region prior to ablation. Electron-ion inverse-bremsstrahlung would therefore dominate the absorption process more quickly than with conventional ablation. Long pulse LIBA is therefore potentially a two-step heating process, with the Al material initially heated as a solid and then further heated as a vapor plume.

Prior to ablation, as laser energy is deposited into the Al film at the interface region, heat can diffuse from this region into the surrounding film regions. This heat diffusion can cause the aluminum in the surrounding regions to melt. The outer phase boundary between the melted $\mathrm{Al}$ region and the outer solid $\mathrm{Al}$ region is called the melt 
front. For LIBA with lower intensity pulses or thinner Al films, one can expect the melt front to pass completely through the thickness of the film prior to ablation ${ }^{13}$. In this case, the plume can be described by the hydrodynamic equations shown below ${ }^{2}$.

$$
\begin{aligned}
& \frac{\partial N}{\partial t}+\frac{\partial}{\partial x}(N v)=0 \\
& \frac{\partial v}{\partial t}+v \frac{\partial v}{\partial x}=-\frac{1}{N m} \frac{\partial p}{\partial x} \\
& \frac{\partial S}{\partial t}+v \frac{\partial S}{\partial x}=0
\end{aligned}
$$

where $\mathrm{S}=\mathrm{pn}^{-\gamma}, \mathrm{N}$ is plume density, $\mathrm{v}$ is plume velocity, and $\mathrm{p}$ is pressure. Assuming ideal gas behavior, $\mathrm{p}=\mathrm{nT}$, and $\gamma=5 / 3$, one finds the self-similar solution for adiabatic decompression is given by Sedov ${ }^{15}$ and is shown below.

$$
\begin{aligned}
N & =N_{0}\left(L_{0} / L\right)=N_{0} y^{-1} \\
T & =T_{0}\left(L_{0} / L\right)^{\frac{2}{3}}\left[1-(x / L)^{2}\right]=T_{0} y^{\frac{-2}{3}}\left(1-\xi^{2}\right) \\
v & =\frac{x}{L} \dot{L}=\xi \dot{y} L_{0} \\
L & =L(t) \\
y & =\frac{L}{L_{0}} \\
\xi & =\frac{x}{L}
\end{aligned}
$$

where $L$ is the density scale length of the plume and $N_{0}, T_{0}$, and $L_{0}$ are values at $t=0$.

Based on this solution, the velocity of the outer plume edge is found to be $\mathrm{v}_{\text {edge }}=\left(6 \mathrm{~T}_{0} / \mathrm{m}\right)^{1 / 2}$. For example, LIBA plumes produced by $970 \mathrm{~nm}$ thick Al films ablated with $1 \mathrm{~ns}, 10^{11} \mathrm{~W} / \mathrm{cm}^{2}$ pulses were shown ${ }^{2}$ to have edge velocities of approximately $7.9 \times 10^{5} \mathrm{~cm} / \mathrm{s}$. According to the solution above, this edge velocity equates to $T_{0} \approx 2-3 \mathrm{eV}$, and for a plume which has expanded to $100 \mu \mathrm{m}$, the temperature at the interface region has dropped to $0.1-0.15 \mathrm{eV}$. 


\subsection{Advantages of Short Laser Pulses in LIBA}

Early studies in LIBA concentrated on using laser systems with long pulse lengths ${ }^{16}$ of order 10 nsecs. While these lasers do produce high-velocity vapor plumes, these plumes show large amounts of instability ${ }^{17}$ and subsequent recollections of plume material demonstrate that these plume can include non-vapor components ${ }^{18}$ such as solid Al flakes and liquid droplets. In this case, back-ablation in the long-pulse regime occurs during the laser pulse and the laser pulse therefore interacts with the expanding Al plume. It is desirable to prevent such a laser/plume interaction, since laser-heating of the expanding plume material likely contributes to the observed instabilities. By shortening the laser pulse, one can minimize the subsequent laser-heating of the ablated plume. For pulses shorter than $10 \mathrm{psec}$, laser interactions can occur on a time scale much less than that of material expansion or ablation. For example, LIBA of a $.5 \mu \mathrm{m}$ film with a 3.33 psec laser pulse causes the plume density to drop from solid density to half solid density on a time scale of order $1 \mathrm{nsec}^{19}$, a timescale approximately 300 laser pulsewidths in length.

Short laser pulses in LIBA also provides additional advantages. When high fluence short laser pulses interact with metals, the high density of conduction electrons at the metal surface restricts energy deposition to an depth of a few skin depths $\left(\delta=(2 /(\mu \sigma \nu \lambda))^{1 / 2}\right)^{20}$. This energy then propagates outside the laser interaction region by heat transport. If the laser pulse length is significantly shorter than the time scale for heat transport, a localized high energy density plasma can form in the laser interaction region. ${ }^{21}$ Plasma pressure can then eject the entire film from the substrate, producing an Al plume of lower edge velocity and temperature than that of longer-pulse LIBA. 
Additionally, with high fluence pulses of less than 10 psec in duration, the incident laser pulse will ionize the substrate as it propagates through the substrate. Sufficiently high laser intensities will produce free electron densities above critical density during the lifetime of the incident pulse. Critical electron density formed by the leading edge of an incident pulse will scatter and reflect the trailing edge of the pulse off-axis and away from the Al film, thus limiting the total transmitted laser fluence delivered to the $\mathrm{Al}$ film. Such a limit would be intensity-dependent, and by manipulating the laser pulse duration, one can dynamically control the transmitted fluence profile and the spatial velocity distribution of the Al plume.

The "Flyer Plate" Concept

In the ideal limit of very short pulses with very high fluence, the interface region which initially absorbs the incident laser energy would become an ionized plasma under extremely high pressure. This pressure would develop on a timescale much shorter than heat diffusion, and at a final ablation pressure, the pressure would blow off the solid metal film from the substrate. The pulse duration would be much shorter than the ablation timescale, and as such, it would interact with a solid target for the entire laser/matter interaction. The outer regions of the Al film away from the interface would then be ejected from the substrate as a highly-localized, solid-phase projectile. One interesting use of picosecond pulse laser technology is to study the viability of flyer plate production.

The Experimental Aims of this Dissertation

Using a high-energy, picosecond laser system with adjustable laser pulse duration, LIBA is studied as a method of $\mathrm{Al}$ plume production. Plumes are characterized based on 
five criteria; edge velocity, divergence, localization, density, and temperature. Modifying various adjustable parameters such as Al film thickness, laser pulsewidth, and incident fluence, I study the LIBA plume production process under differing conditions. The purpose of this study is to understand short-pulse LIBA as both a physical process and as a practical $\mathrm{Al}$ plume production technique. For this reason, this dissertation discusses both the physical characteristics of LIBA and the practical details of controlled Al plume production.

This dissertation begins with an examination of the laser and diagnostic techniques used to study picosecond pulse LIBA (chapter 2). Since this pulse regime is new to LIBA, it is important to detail the laser technology which allows the production and diagnosis of picoseond laser pulses. Also, descriptions of both the spectrometer and shadowgraph/interferometry systems are included so the experimental observations discussed later can be better understood. After the experimental discussion, the observations of the experiment are discussed (chapter 3). LIBA plume characteristics such as edge velocity and divergence are studied using both interferograms and shadowgraphs, while time-integrated images and time-integrated emission spectroscopy are used to probe ionization and excitation of both the Al film and the glass substrate. Also, a post-experimental study of the $\mathrm{Al}$ target and the recollected $\mathrm{Al}$ plumes is also discussed to further understand LIBA dynamics. A deeper analysis of the observations listed previously is included (chapter 4). Calculations of the total transmitted fluence through a laser-ionized substrate front-surface are shown to agree with the measured transmitted energy through an uncoated BK-7 substrate, and the calculation also shows a relationship between observed edge velocity limits and calculated substrate transmission 
limits. A detailed calculation of plume edge velocity, temperature and pressure is done with the HYADES hydrodynamics and energy-transport code, and these calculations are used to reveal plume characteristics in early time, when experimental probing is more difficult. An analysis of $\mathrm{Al}$ plume temperature using time-integrated spectra is also included, as well as a discussion of the practical aspects of Al plume production. Finally, this dissertation concludes with a discussion of the major points of this experimental study (chapter 5). Suggestions for future exploration of this process are also included.

\footnotetext{
${ }^{1}$ J.C.S. Kools, Pulsed Laser Deposition of Thin Films, edited by D.B.Chrisey and G.K. Hubler (Wiley, New York, 1994), p.456.

${ }^{2}$ F.J. Mayer and Gar. E. Busch, J. Appl. Phys. 57, 827 (1985).

${ }^{3}$ P.R. Bolton, D.C. Eder, G. Guethlein, R.E. Stewart, and P.E. Young, Proc. SPIE 1890, 167 (1993).

${ }^{4}$ R. Wangness, Electromagnetic Fields, John Wiley and Sons, New York, p.423 (1986).

${ }^{5}$ S.A. Akhmanov, V.I. Emel'yanov, N.I. Koroteev, and V.N. Seminogov, Sov. Phys. Usp. 28(12), 1985.

${ }^{6}$ H. Kurz, L.A. Lompre, and J.M.Liu, J. Phys. (Paris) 44, Suppl. Nr. 10, C5-23(1983).

${ }^{7}$ A. Peterlongo, A. Miotello, and R. Kelly, Phys. Rev E. 50, p.4716 (1994).

${ }^{8}$ R. Kelly and A. Miotello, "Mechanisms of Pulsed Laser Sputtering"in Pulsed Laser Deposition of Thin Films, D.B. Chrisey and G.K. Hubler, eds. p.64 (1994).

${ }^{9}$ R. Kelly and J.E. Rothenberg, Nucl. Instrum. Meth. B7/8, p.755 (1985).

${ }^{10}$ E. Oktay and D.R. Bach, J. Appl. Phys. 41, 1716 (1970).

${ }^{11}$ Y. B. Zeldovich and Y.P. Raizer, Physics of Shock Waves and High-Temperature Hydrodynamic Phenomena, 1 p. 386.

${ }^{12}$ D.J. Heading, G.R. Bennett, J.S. Wark, and R. W. Lee, Phys. Rev. Lett. 74, 3616 (1995).

${ }^{13}$ F. J. Adrian, J. Bohandy, B.F. Kim, A.N. Jette, and P. Thompson, J. Vac. Sci. Technol. B5, 1490 (1987).

${ }^{14}$ A.B. Bullock, P.R. Bolton, and Fred J. Mayer, J. Appl. Phys. 821828 (1997).

${ }^{15}$ L.I. Sedov, Similarity and Dimensional Method in Mechanics, (Academic, New York, 1959) p. 271.

${ }^{16}$ F.J. Mayer and Gar. E. Busch, J. Appl. Phys. 57, 827 (1985).

${ }^{17}$ A.B. Bullock, P.R.Bolton, and F.J. Mayer, J. Appl. Phys. 82, 1828 (1997).

${ }^{18}$ J. Bohandy, B.F. Kim, and F.J. Adrian, J. Appl. Phys. 60 (4), 1538 (1986).

${ }^{19}$ A.B. Bullock and P.R. Bolton, Submitted to High-Power Laser Ablation Conference, Proc. SPIE.

${ }^{20}$ R.W. Wangness, Electromagnetic Fields (Wiley, New York, 1986), p. 384.

${ }^{21}$ A.M.Rubenchik, M.D.Feit, M.D.Perry, and J.T.Larsen, Submitted to Applied Surface Science COLA'97, July 21-25, 1997, Monterey, Ca.
} 


\section{CHAPTER 2: EXPERIMENTAL METHOD}

\subsection{Overview}

The short-pulse LIBA process was studied with the method described below (see figure 2.1.1). A high-fluence $\left(1-160 \mathrm{~J} / \mathrm{cm}^{2}\right)$, short duration (1.85 or $3.33 \mathrm{ps}$ FWHM gaussian) ablation pulse $(1053 \mathrm{~nm})$ is focused and after passing through the focal point is propagated into the front surface of an $\mathrm{Al}$ target. The $\mathrm{Al}$ target consists of a BK-7 glass substrate (thickness $\mathrm{t}=1.2 \mathrm{~mm}$ ) coated on the back surface with a $0.1 \mu \mathrm{m}, 0.25 \mu \mathrm{m}$, or 0.5 $\mu \mathrm{m}$ Al film. The ablation pulse propagates through the front surface of the substrate and into the Al film at the substrate/film interface. The ablated $\mathrm{Al}$ plume is then forced from the back surface of the substrate to velocities of order $10^{5} \mathrm{~cm} / \mathrm{s}$. The two-color probe pulse (1053nm and $527 \mathrm{~nm}$ ), after passing through an optical delay line (255 ns or $320 \mathrm{~ns}$ delay relative to the arrival of the ablation pulse), transversely probes the Al plume and then passes into a two-color, self-referencing interferometer, thereby producing images of the $\mathrm{Al}$ plumes in flight.

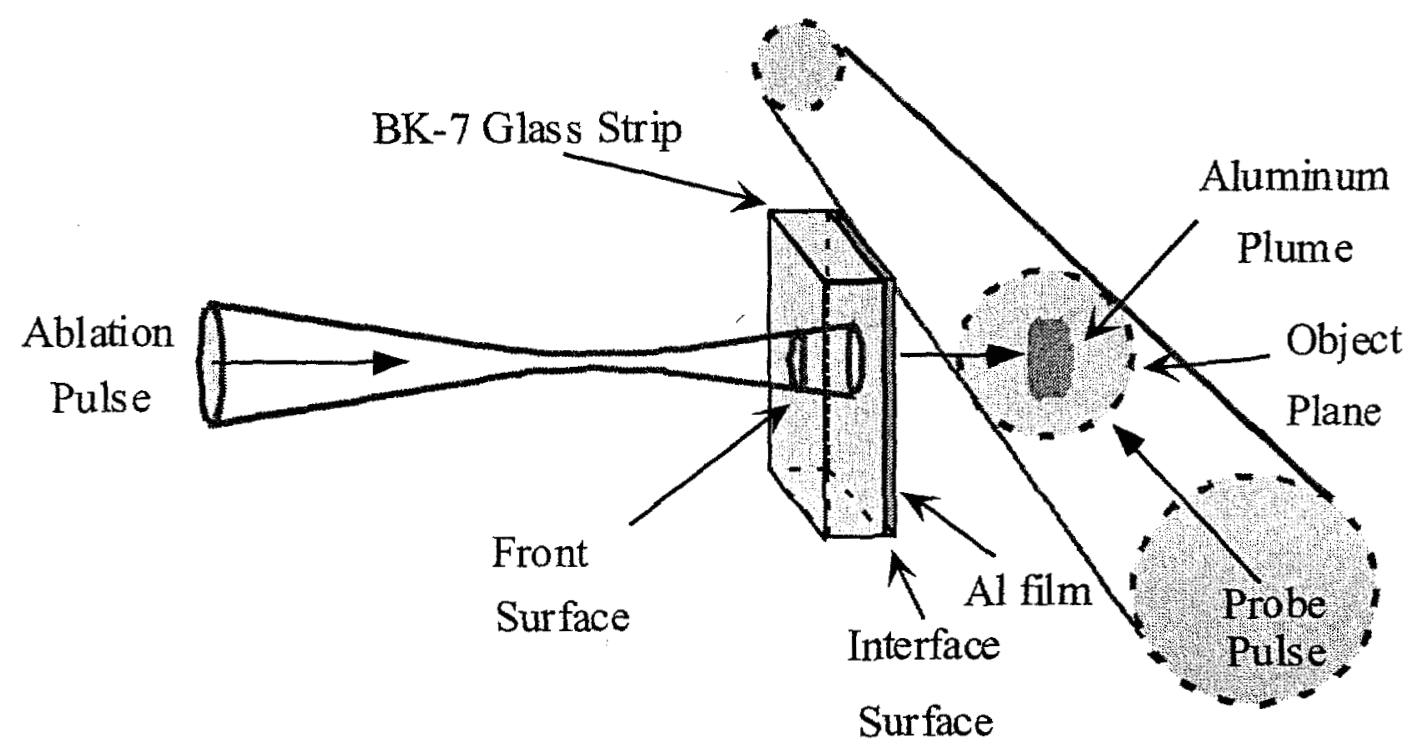

Figure 2.1.1. Diagram of LIBA interaction scheme. 
Laser pulses are initially generated by the Picosecond laser system at the Janus laser facility in V-division of LLNL (see appendix A). Each pulse is then sent through the apparatus shown in figure 2.1.2. The main ablation pulse is recompressed by a vacuum

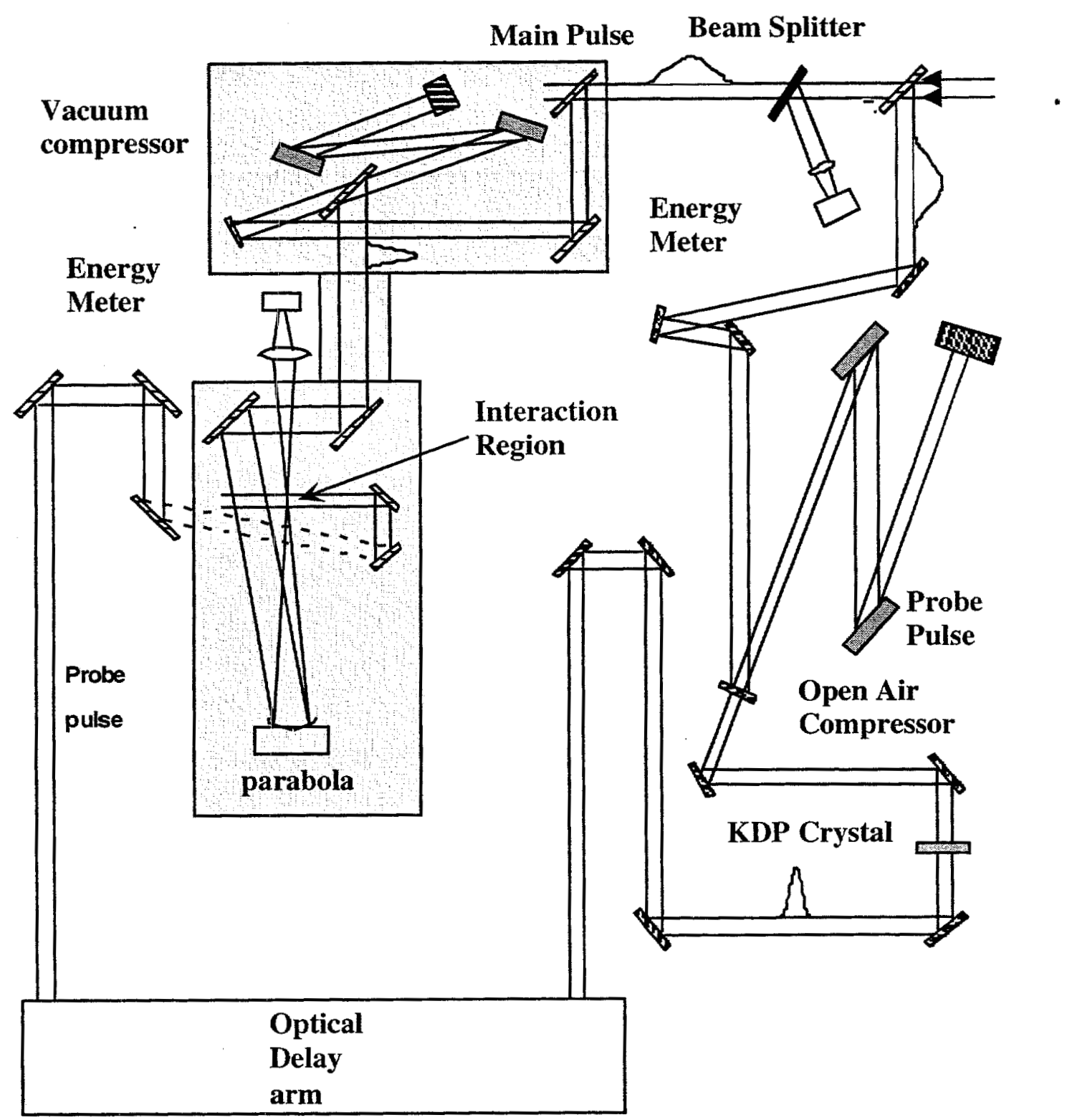

Figure 2.1.2. Diagram of experimental apparatus used to direct picosecond pulses into vacuum chamber. 
compressor, and directed through a series of mirrors to a focusing parabola, where it is directed onto the Al target. A small part of each pulse is redirected prior to recompression for use in energy sampling. Another small portion of the main pulse is redirected through a smaller open-air compressor system and sent through a KDP crystal to produce a twocolor probe pulse. This probe pulse is directed through an optical delay arm and then transversed the ablated Al plume. An energy meter (J-25 molectron power head) is placed outside the vacuum chamber directly behind the Al target position to measure transmitted energy through uncoated BK-7 substrates placed in the Al target position. Laser pulsewidth is measured by a single-shot intensity autocorrelator (see appendix B).

In addition to the two-color probe pulse, time-integrated images of the optical emission from the $\mathrm{Al}$ target and ejected plume are recorded by a CCD camera placed 90 degrees from the incident ablation pulse and 30 degrees from the horizontal plane. These images show both the in-flight emissions of the Al plume and also the emissions associated with the glass ionization which occurs at sufficiently high laser fluence. Also, the optical spectra of plume emission is collected during LIBA by a grating spectrometer operating in the visible region. This system allows the study of the time-integrated emission spectra attributed to both the Al plume and damage at the glass surface.

Aluminum targets are comprised of a BK-7 glass strip (thickness $t=0.94 \mathrm{~mm}$ ) with an $\mathrm{Al}$ film $(\mathrm{t}=0.1 \mu \mathrm{m}, 0.25 \mu \mathrm{m}$ and $0.50 \mu \mathrm{m})$ on the rear surface. To aid adhesion, the glass strip is exposed to a $200 \mathrm{~mA}, 750 \mathrm{~V}$ glow discharge for $30 \mathrm{sec}$ prior to $\mathrm{Al}$ film deposition. This discharge cleans the organic contamination off the substrate surface. Aluminum is then deposited on the rear strip surface by electron beam evaporative coating (average rate $\mathrm{r}=5 \AA / \mathrm{s})$. 


\subsection{Two-Color Self-Referencing Interferometer}

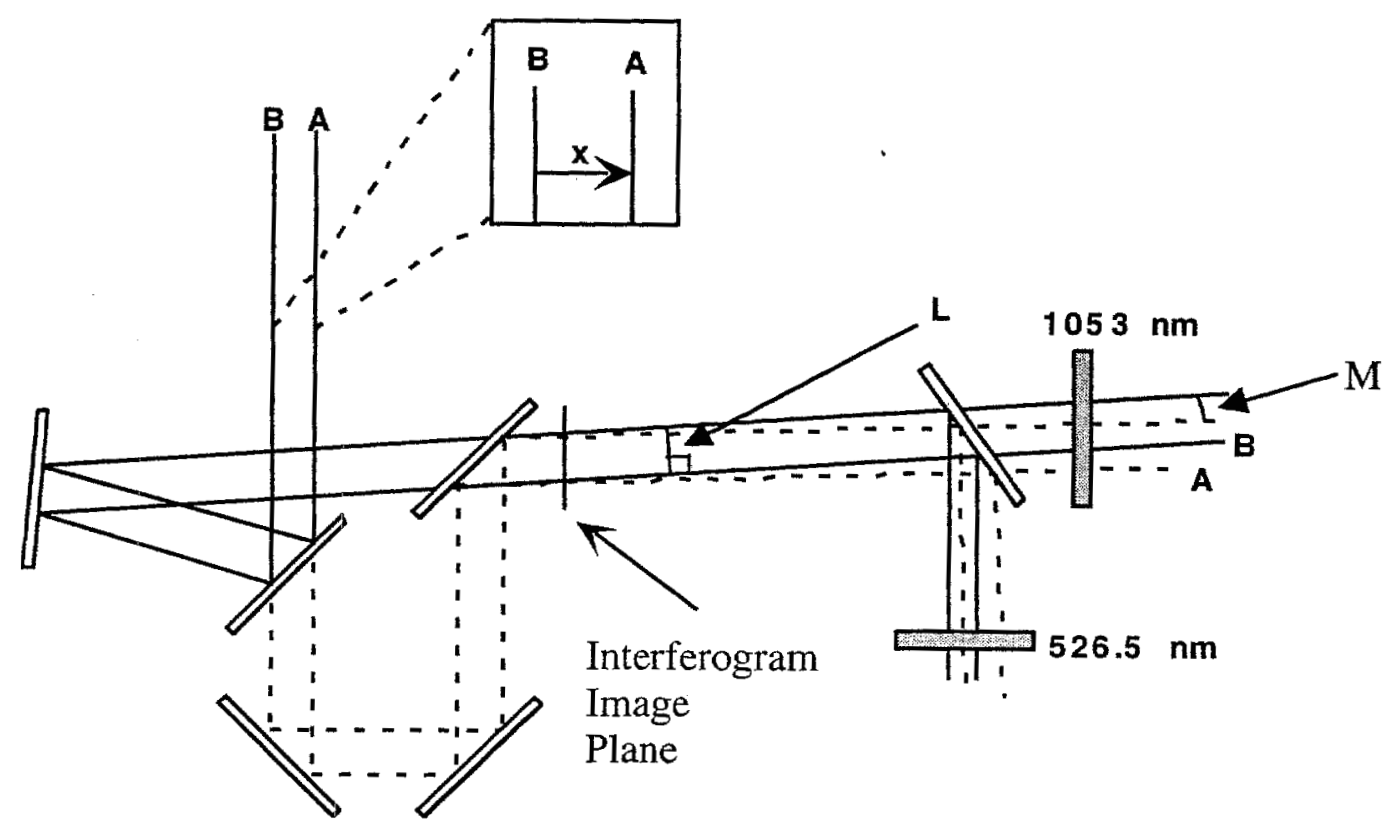

Figure 2.2.1 Diagram of two-color self-referencing interferometer.

One of the primary LIBA diagnostics in this experiment is the two-color imaging interferometer. The diagnostic is described below. The time-delayed probe is directed through the ablated metal plume and into a self-referencing interferometer. This interferometer produces a fringe pattern on the incoming beam that represents the spatial phase variations of the incoming beam. By imaging the plume with a two part lens array, the image of the plume overlaid with a fringe pattern is gathered by a CCD camera. Phase shifts induced in the probe beam by the plume are clearly represented in the CCD images. Since neutral atoms and electrons cause well-understood phase shifts and these phase shifts are differently related to wavelength, a two-color probe pulse can produce two 
interferograms which, together, map the neutral and electron density as a function of position through the plume.

The self-referencing interferometer initially separates the incoming probe pulse into two daughter pulses (see figure 2.2.1). One daughter pulse is shown in figure 2.2.1 as the solid line, while the other pulse is shown as a dotted line. The solid beam is reflected off the first beam splitter and then off a simple mirror, while the dotted beam is reflected off one beam splitter and two simple mirrors. The two beams are recombined after the final beam splitter, and since the dotted beam and solid beam are slightly non-collinear, the solid beam wave vector, $k_{1}$, and the dotted beam wave vector, $k$, form the small angle, M, between them. This angle difference produces a sinusoidal fringe pattern. Since the dotted beam is reflected from one more mirror than the solid beam, the dotted beam has a phase profile which is inverted horizontally relative to the solid beam. If one adds the field produced at the interferogram image plane by the solid beam, $E(x) \exp \left(i \vec{k}_{1} \bullet \vec{r}-i \omega t\right)$, and the field produced by the dotted beam, $E(L-x) \exp (i \vec{k} \bullet \vec{r}-i \omega t)$, where $L$ is the transverse width of the probe beam, one arrives at the expression for the intensity shown below.

$$
\begin{aligned}
& I(x)=|E(x)|^{2}+|E(L-x)|^{2}+E(x) E^{*}(L-x) \exp \left(i\left(\vec{k}_{1}-\vec{k}\right) \vec{r}\right) \\
& +E^{*}(x) E(L-x) \exp \left(-i\left(\vec{k}_{1}-\vec{k}\right) \vec{r}\right) \quad(2.1)
\end{aligned}
$$

In the case above, a 1-D expression is shown. However, the argument expressed in (2.1) is equally valid in the $\mathrm{x}-\mathrm{y}$ plane. If one now assumes the beam has a planar phase front ( i.e.: the phase is everywhere identical in a given plane), one can let $\mathrm{E}(\mathrm{x})$ equal $E(x)=A(x) \exp (i B)$, where $A$ is real and $B$ is a constant. Inserting this into (2.1) and 
allowing the longitudinal distance from the final mirror to be represented by $z$, one gets the expression below.

$$
I(x)=|A(x)|^{2}+|A(L-x)|^{2}+2 A(x) A^{*}(L-x) \operatorname{Cos}\left(\left(k_{1 x}-k_{x}\right) x+\left(k_{1 z}-k_{z}\right) z\right)
$$

Now, if, when propagating through the plume, the probe undergoes a local phase shift, one can represent this as a new expression for the field, $E(x)=A(x) \exp (i B+i G(x))$, where $\mathrm{G}(\mathrm{x})$ is a function of transverse position. This alters the expression (2.2), producing the expression seen below.

$$
\begin{aligned}
& I(x)=|A(x)|^{2}+|A(L-x)|^{2} \\
& +2 A(x) A^{*}(L-x) \operatorname{Cos}\left(\left(k_{1 x}-k_{x}\right) x+\left(k_{1 z}-k_{z}\right) z+G(x)-G(x-L)\right)
\end{aligned}
$$

As one can see in (2.3), when the plume induces a phase shift in the probe beam, the sinusoidal fringe pattern will shift position. Since a two-lens array images the plume directly into the CCD camera, a image of the plume overlays the fringe pattern. The total CCD image is that of a new fringe pattern formed by the coherent combination of the two field patterns. Thus, for all points for the plume which are imaged along a fringe, there is a definite phase shift value.

This measured phase shift results from probe propagation through the plume. When the laser passes through the plume, the neutral atoms, free electrons, and other species interact with the field. Since LIBA plumes can be expected to exhibit very low temperature $(\mathrm{T}<<1 \mathrm{eV})$ at delays of order $200 \mathrm{~ns}$, it is reasonable to expect ion density and excited neutral atom density to be far lower than that of ground-state neutral density. In that case, ground-state neutral atoms and free electrons will dominate over other phaseshifting contributions. Ground-state neutrals produce phase-shift because the finite 
polarization of each atom interacts with the electric field of the probe pulse as it passes through the plume. With a large number of neutral atoms, this effect can be expressed with the Lorentz-Lorenz relation'. Figure 2.2.2 shows one element of the probe passing through a length of plume, $T$, and if one calls the average neutral atom density along this path $n_{n}$, then the phase shift induced on the probe pulse element due to neutral atoms is $\Delta \phi \approx 4 \pi^{2} N \gamma_{A l} T / \lambda$, where $\gamma_{\mathrm{Al}}$ is the atomic polarizability of aluminum ${ }^{25}$.

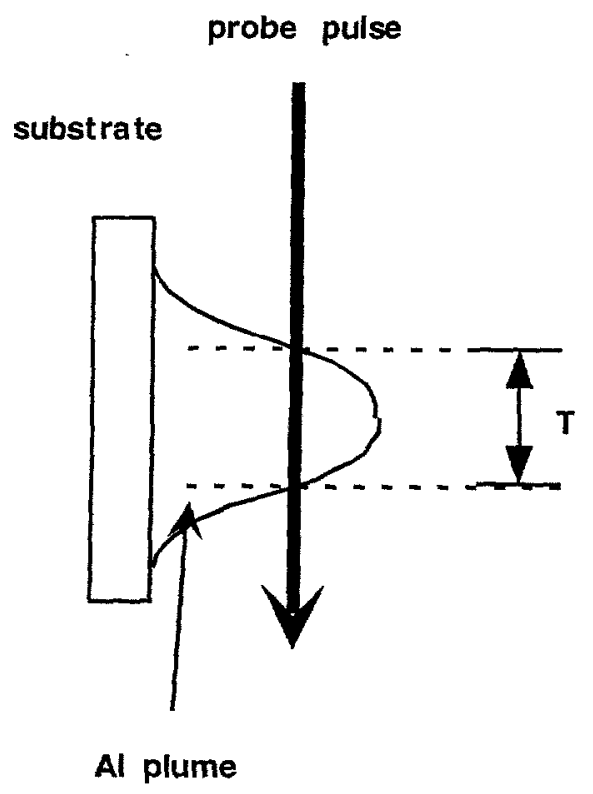

Figure 2.2.2. Diagram of plume interaction length, $\mathrm{T}$.

Similarly, the presence of free electrons causes changes in the index of refraction, which in turn cause phase shifts in the probe pulse. This phase shift can be expressed as $\Delta \phi \approx-n_{e} T \lambda \frac{e^{2}}{m c^{2}}$, where $\mathrm{n}_{\mathrm{e}}$ is the average electron density along the probe path $^{2}$.

Together, the sum of phase shifts for both neutral atoms and electrons can be expressed by the expression $\Delta \phi_{\text {total }}=D N / \lambda+E n_{e} \lambda$, where $\mathrm{D}=4 \pi^{2} \gamma_{\mathrm{Al}} \mathrm{T}$ and $\mathrm{E}=\mathrm{e}^{2} \mathrm{~T} / \mathrm{mc}^{2}$. Since the phase shift for free electrons has a different wavelength dependence than that of the 
neutral atoms, a two-color probe pulse can be used to separate out the phase shift components for both neutrals and electrons. Our experiment uses a two-color probe $(1053 \mathrm{~nm}$ and $526.5 \mathrm{~nm})$, and figure 2.2.1 shows the line filters which allow separation of one color from another. This allows the system to gather two interferograms, one for each color. Expressions for each color are shown below.

$$
\begin{gathered}
\Delta \phi_{526.5 n n}=2 D n_{n} / \lambda_{1053}+\frac{1}{2} E n_{e} \lambda_{1053} \\
\Delta \phi_{1053 n m}=D N / \lambda_{1053}+E n_{e} \lambda_{1053}
\end{gathered}
$$

These equations can be solved for the neutral and electron densities, and the solution is shown below.

$$
\begin{aligned}
& n_{t}(x) \approx-\frac{2}{3 E \lambda_{1053}}\left[\Delta \phi_{526.5}(x)-2 \Delta \phi_{1053 n m}(x)\right] \\
& N(x) \approx \frac{\lambda_{1053}}{3 D}\left[2 \Delta \phi_{526.5}(x)-\Delta \phi_{1053 n m}(x)\right]
\end{aligned}
$$




\subsection{Time-Integrating Spectrometer}

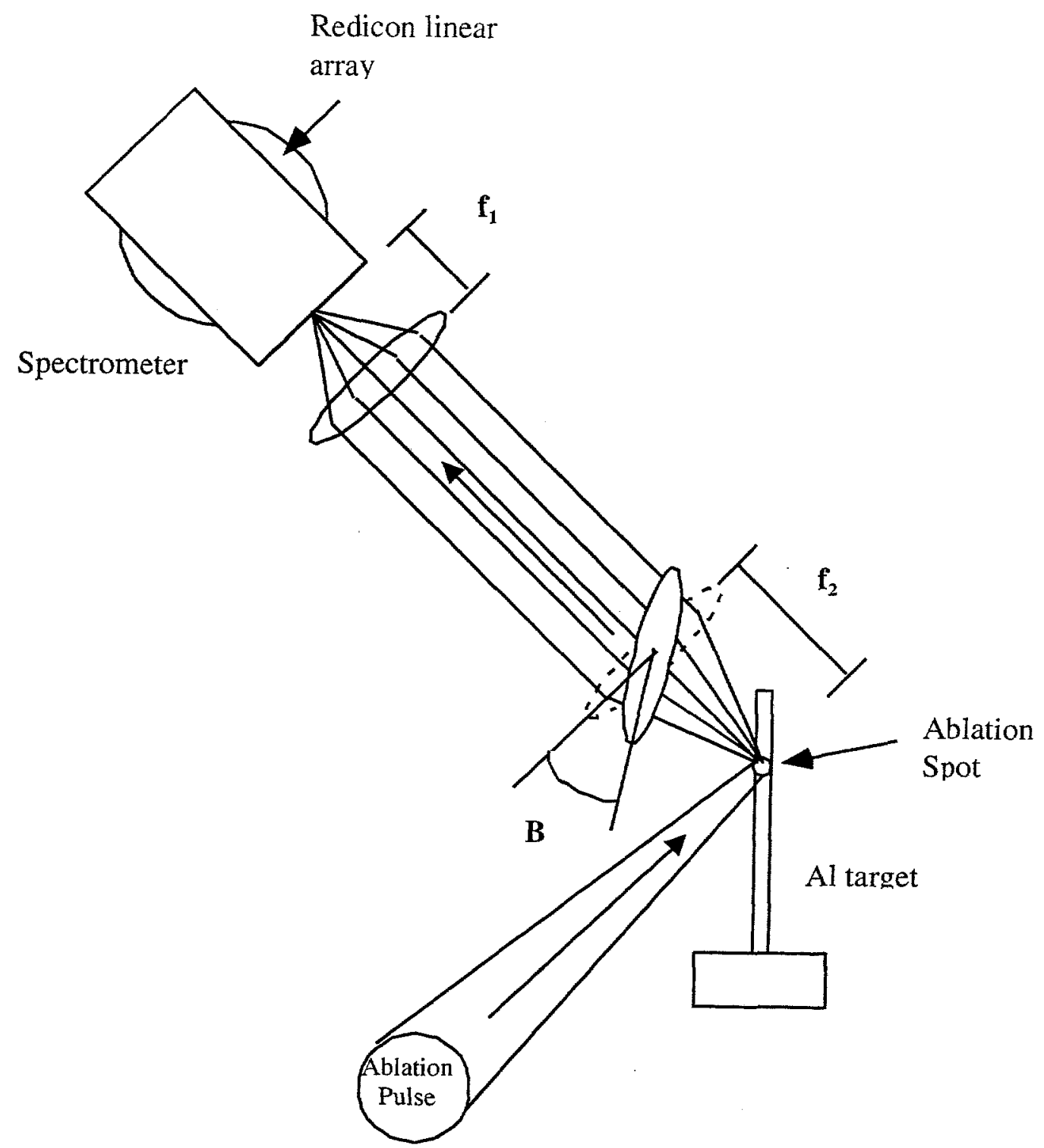

Figure 2.3.1 Diagram of time-integrating spectrometer system

During back-ablation, both the glass substrate and the aluminum plume emit visible light. These emissions can reveal important information about the ionization state and temperature of the ablated plume, as well as the laser-damaged front surface. I studied these emissions with a time-integrated spectrometer system which is described below(see Figure 2.3.1). 
A lens $(f=5.02 \mathrm{~cm})$ is placed at a distance, $f_{2}=4 \mathrm{~cm}$, from the ablation spot. Another lens $(f=10 \mathrm{~cm})$ is placed outside the chamber at a distance, $f_{1}=8 \mathrm{~cm}$, from the entrance slit of a spectrometer. The purpose of the two lens configuration is to collimate the light emitted from the ablation event, transmit the light outside the chamber and focus it into the spectrometer. Due to space constraints imposed by the focusing elements inside the vacuum chamber, however, the first lens is not placed normal to the desired collimated light path but instead placed at a $32^{\circ}$ angle, B, off normal. This prevents the two lens system from exactly collimating the light emission. The incoming light level from the region of interest was maximized by adjusting the lens position. This light passes through a $50 \mu \mathrm{m}$ entrance slit of the spectrometer. The spectrometer diffracts the incoming signal into spectral components with a diffraction grating (see below), and each component is then imaged on to the $1455 \mathrm{~A}$ redicon amplified linear detector array. The redicon then integrates the signals over a timescale of order milliseconds and transmits the results to a Macintosh computer for analysis.

The two lens system, in combination with the $50 \mu \mathrm{m}$ slit, accepts optical emission from a small area near the $\mathrm{Al}$ target. The lens array is set such that the emission collection area is centered at the thin film side of the Al target in the center of the ablation laser spot. Emission away from the ablation spot is not collected with the same efficiency as the emission in the ablation spot area. By transversely illuminating an Al target with a $\mathrm{HeNe}$ laser $(623 \mathrm{~nm})$, and scanning the $\mathrm{Al}$ target along the ablation pulse propagation axis (z-axis), the relative collection efficiency was measured as a function of axial position. The relationship between $\mathrm{z}$-axis position and relative detection efficiency is shown in figure 2.3.2. By positioning the lens array to maximize this efficiency on the 
back surface of the Al target, one reduces the efficiency at the front surface to one-third that of the back surface. This allows the preferential gathering of Al line emission in the presence of front surface Si line emission.

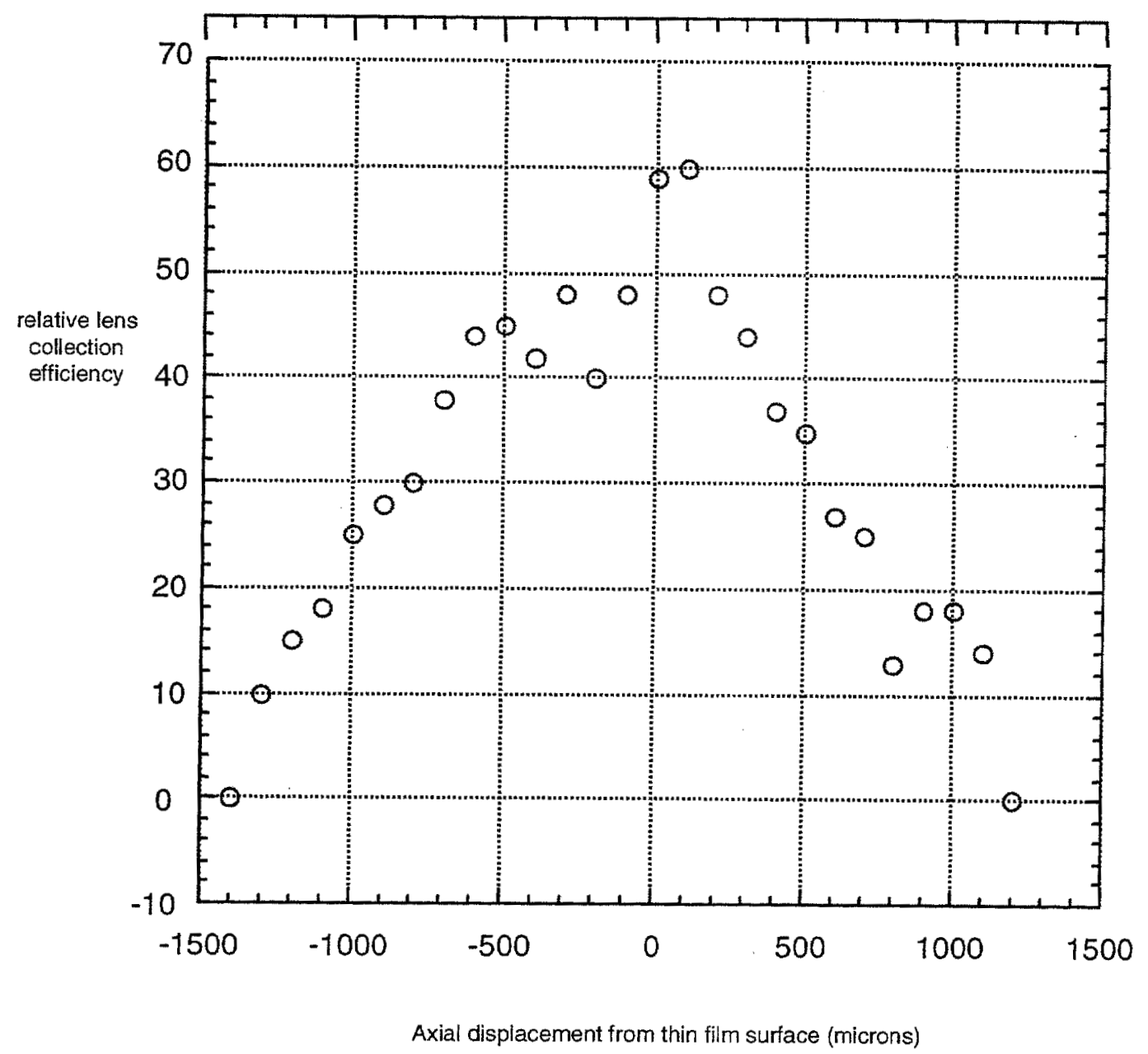

Figure 2.3.2 Dependence of HeNe signal scattered from Al target on Al target axial position.

The spectrometer has a 300 lines/mm, $500 \mathrm{~nm}$ blazed diffraction grating. This results in a dispersion described by $\mathrm{d} \theta / \mathrm{d} \omega=-2 \pi \mathrm{c} / \omega^{2} \mathrm{~d}$, where $\mathrm{d}$ is the grating constant. The 
focusing mirrors in the spectrometer image the illuminated $50 \mu \mathrm{m}$ slit onto the 1012 element redicon array. The combination of these three factors produces a wavelength scale of approximately $0.51 \mathrm{~nm} /$ pixel. Since it would require 3 pixels to resolve two different spectral lines, the redicon places a resolution limit of $1.53 \mathrm{~nm}$ on the spectrometer system. In practice, experimental conditions limit spectrometer resolution to approximately $3 \mathrm{~nm}$. The redicon also has an observation window from $450 \mathrm{~nm}$ to 850 $\mathrm{nm}$. Back-ablation spectra was obtained by dividing out the combined wavelength sensitivities of the redicon detector, the lens and window array, and the diffraction grating.

${ }^{1}$ J. D. Jackson, Classical Electrodynanics, p. 155.

${ }^{2}$ Ibid., pp.288-289. 


\section{CHAPTER 3:EXPERIMENTAL OBSERVATIONS DURING LIBA}

\subsection{Introduction}

During this experimental study of LIBA, the diagnostics described in chapter 2 are all used to observe the ablation event. Additionally, a time-integrating (1/30 second resolution) Hitachi KP-140 CCD video camera and an Oriel 77340 PMT (time resolution $1 \mathrm{~ns}$ ) are included to record the time-integrated image of plume emission and the time history of the spectrally-integrated emission, respectively. Using these instruments, several important observations are made about short pulse LIBA. Optical emissions from the ablated $\mathrm{Al}$ plume show the flight path of the ejected material during LIBA, and for higher incident laser intensities, optical emissions from the substrate front surface indicate laser-induced ionization of the substrate. Measurements of transmitted laser energy through BK-7 substrate substrates show that laser-induced substrate damage limits laser fluence transmission to the Al thin film on the back surface. Shadowgraphs and interferograms of the ejected $\mathrm{Al}$ plume reveal low plume divergence and a well-defined plume edge. The velocity of this edge can be used to relate plume velocity to incident laser fluence. Low-fluence edge velocity shows a functional form which suggests the existence to a laser-ablation threshold, and high fluence edge velocity data indicates that edge velocity growth saturates at some transition point. Two-color interferometric measurements of electron and neutral density for ablated $0.1 \mu \mathrm{m}$ Al films show that at $255 \mathrm{~ns}$ after the arrival of the ablation pulse at the Al target, the plumes are weakly ionized and are significantly more divergent than that of plumes produced from $0.25 \mu \mathrm{m}$ and $0.5 \mu \mathrm{m}$ films. Observations of spectral emissions show that the Al plumes have a low 
temperature and Si spectral lines, indicative of substrate damage, become very prominent at high laser fluence.

\subsection{Laser-Induced Substrate Damage}

We readily observe laser-induced ionization and breakdown of substrates during picosecond pulse LIBA experiments. Figure 3.2.1 shows an oblique angle CCD camera image of laser-induced front surface breakdown during LIBA. These observations are consistent with published estimates of the breakdown threshold fluence $\left(\mathrm{J}_{\mathrm{th}} \approx 2.5 \mathrm{~J} / \mathrm{cm}^{2}\right)$ for $1053 \mathrm{~nm}$ pulses of duration $1.85 \mathrm{ps}$ in fused silica ${ }^{1}$

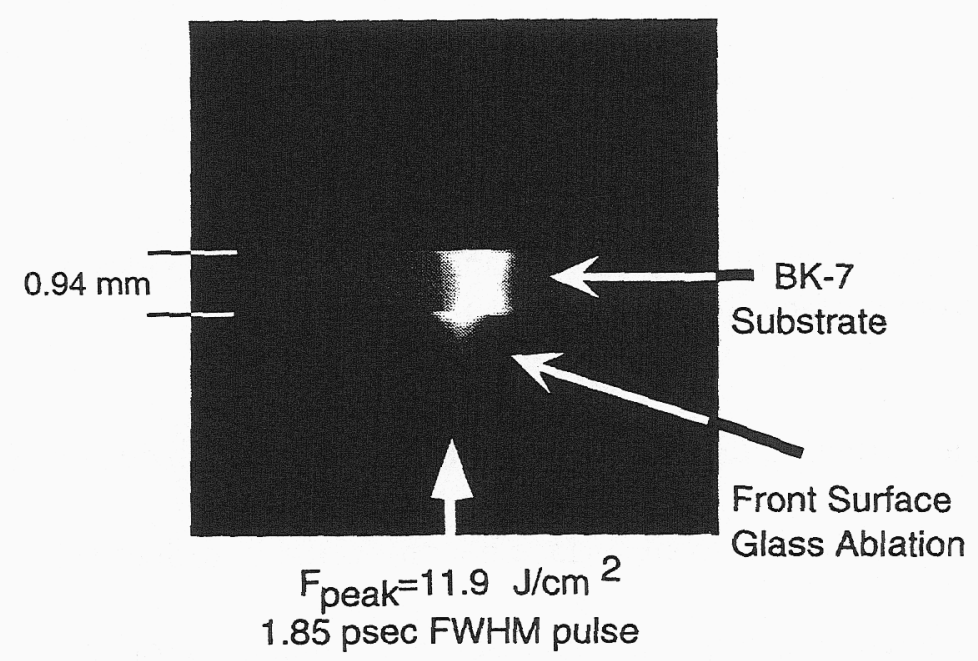

Figure 3.2.1. Oblique angle $C C D$ camera image of uncoated $B K-7$ substrate during LIBA. Note the laser-induced ionization on front substrate surface.

According to a simple model discussed in the chapter 4 , above this fluence, the laserinduced electron density at the substrate surface exceeds critical electron density 


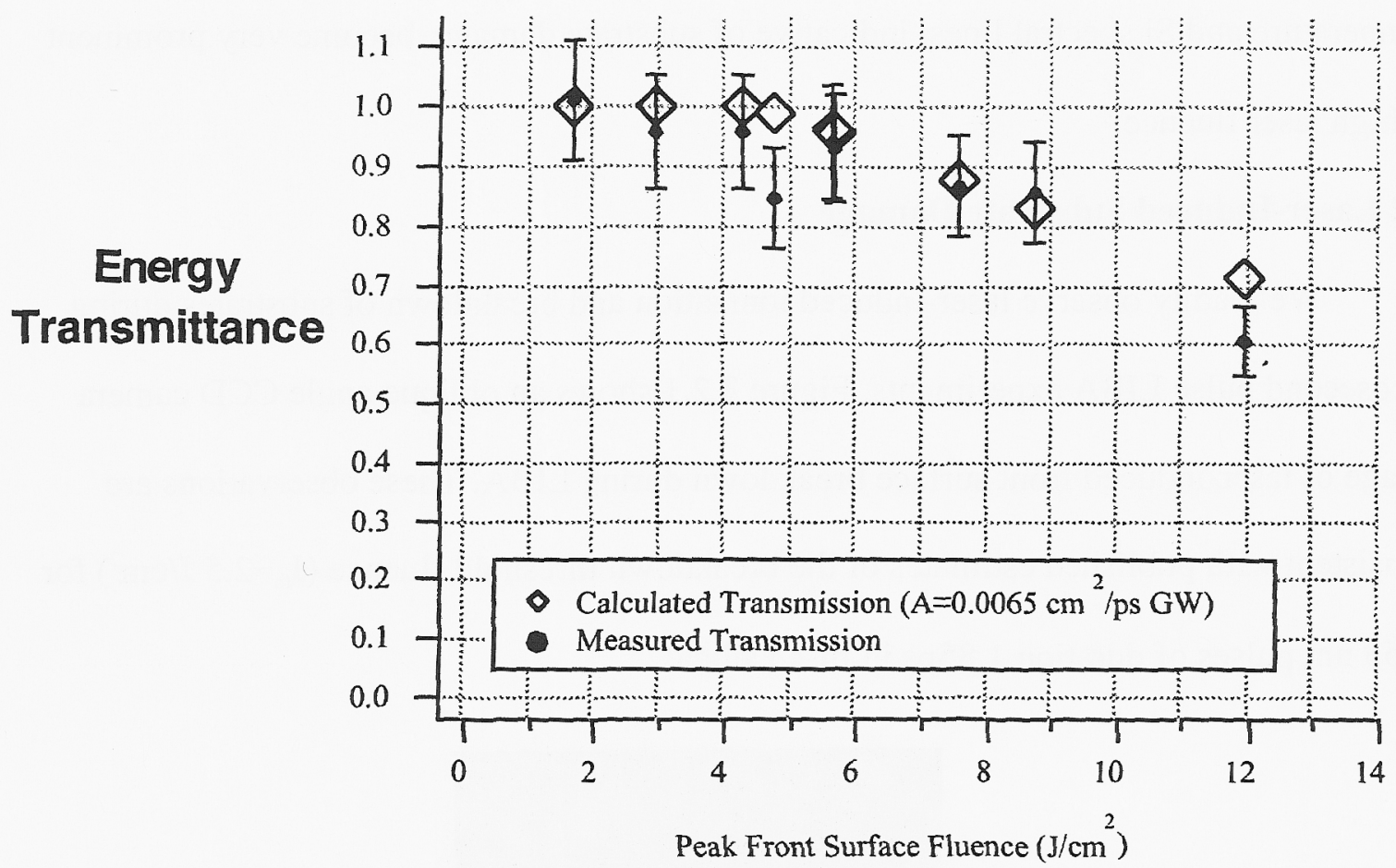

Figure 3.2.2. Dependence of the ratio of transmitted energy to incident laser energy on peak incident front surface fluence for 1.85 ps laser pulses.

$\left(\mathrm{n}_{\mathrm{cr}} \approx 10^{21} \mathrm{~cm}^{-3}\right)$ at a time, $\mathrm{t}_{0}$, during the laser pulse. After the electron density exceeds critical density, the substrate front surface becomes highly reflective, and the portion of the incident pulse which arrives after some critical time, $\mathrm{t}_{0}$, is reflected away from the substrate. This latter portion of the incident pulse does not propagate into the Al film, and therefore, laser-induced ionization limits the total transmitted laser fluence which reaches the Al film on the back surface.

We can study this effect by measuring the total transmitted energy through an uncoated BK-7 substrate slide as a function of laser pulse duration and fluence. Laser energy transmission though uncoated $\mathrm{BK}-7$ substrate was studied with $\mathrm{BK}-7$ substrates (thickness $\mathrm{t}=0.94 \mu \mathrm{m}$ ) similar to those used to make $\mathrm{Al}$ targets. The $\mathrm{Bk}-7$ targets are placed at approximately the same position used for Al targets (with laser spot size 
$A=6.8 \times 10^{-3} \mathrm{~cm}^{2}$ on the front surface). Transmitted laser energy was collected by a $\mathrm{f} / 5.3$ lens placed $84.8 \mathrm{~cm}$ behind the target and directed into a pyroelectric energy meter (Molectron J-25 power head). This data is shown in figure 3.2.2 and figure 3.2.3.

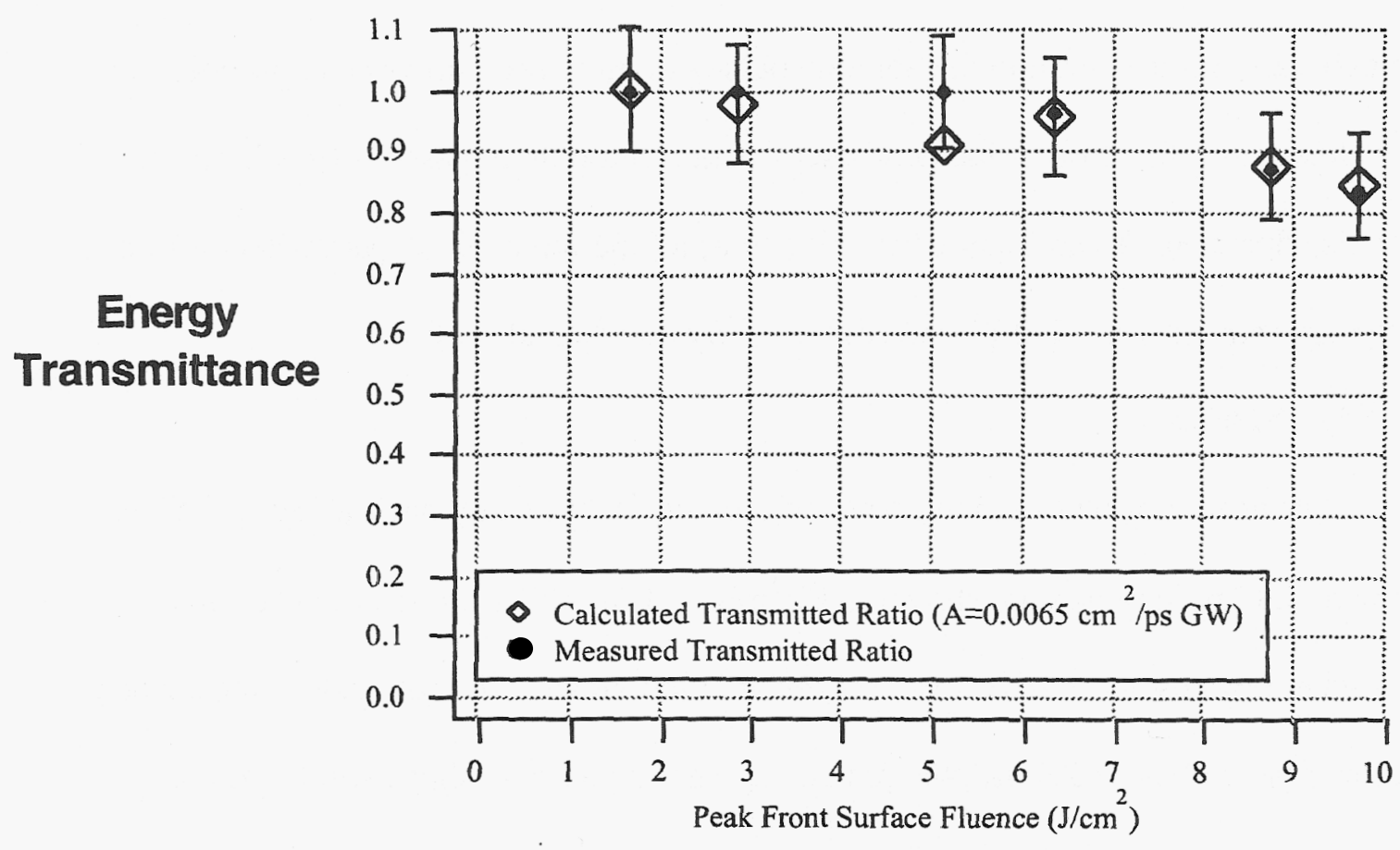

Figure 3.2.3. Dependence of the ratio of transmitted energy to incident laser energy on peak incident front surface fluence for 3.33 ps laser pulses.

\subsection{Edge Velocity Measurements Using Shadowgraphs and Interferograms}

Plumes formed by picosecond LIBA can have well-defined outer edges, and these edges form the boundary between the high-density plume region and vacuum. One of the important dynamic features of LIBA-generated plumes is, therefore, the observed plume edge velocity. Although interferograms and shadowgraphs of ablated plumes readily show this outer edge, detailed features in the interior of the plume are not as easily observed. For this reason, we use the leading plume edge as an important experimental 
observable. By recording shadowgraphs and interferograms at specified delays after the ablation event, we can then obtain spatial edge velocity profiles along one transverse dimension. Mapping the incident laser spatial beam profile over the edge velocity profile, we study the dependence of the edge velocity on incident laser fluence. Changing the aluminum target thickness and the ablation pulse duration also allows an exploration of edge velocity as a function of incident laser fluence and plume mass for two different laser pulse durations. Examining edge velocity as a function of these parameters affords the experimentalist control of plume edge velocity and spatial profile.

\subsubsection{Edge Velocity}

Interferograms and shadowgraphs of plumes generated by back-ablating $0.50 \mu \mathrm{m}$ films with 3.33 psec pulses are shown in Figure 3.3.1.1. In this figure the delay time is $255 \mathrm{~ns}$ where $\mathrm{t}=0 \mathrm{~ns}$ is the arrival time of the ablation pulse at the substrate front surface. With low fluence below $5 \mathrm{~J} / \mathrm{cm}^{2}$, the plume edge has a gaussian shape similar to the incident beam profile. At high fluences such as $11.9 \mathrm{~J} / \mathrm{cm}^{2}$, however, the edge is almost completely blunted and takes on a 'flat-top' spatial profile. A similar effect is shown in figure 3.3.1.2 for $0.50 \mu \mathrm{m}$ films with $1.85 \mathrm{psec}$ pulses, although the blunting effect results in a noticeably slower edge profile. 

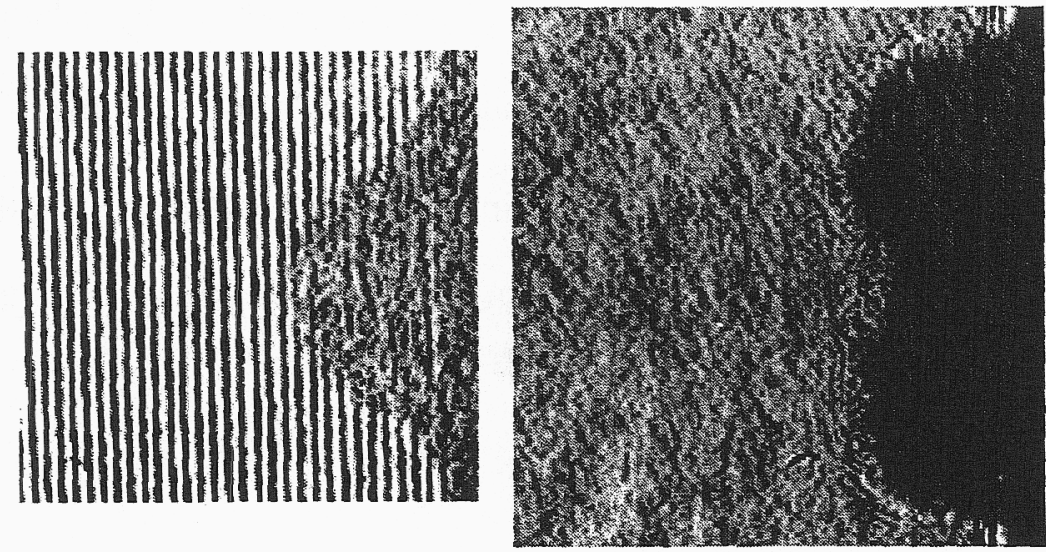

$$
\mathrm{J}_{\text {peak }}=4.68 \mathrm{~J} / \mathrm{cm}^{2}
$$

$$
J_{\text {peak }}=11.9 \mathrm{~J} / \mathrm{cm}^{2}
$$

Figure 3.3.1.1. Interferograms and shadowgraphs showing LIBA-generated plumes produced by 3.33 ps pulses ablating $.50 \mu \mathrm{m}$ films. Each plume was produced with a differently sized incident beam profile. Each interferogram was captured $255 \mathrm{~ns}$ after the ablation pulse.

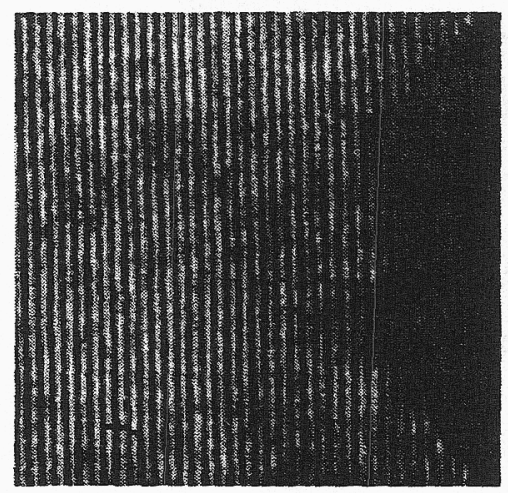

theak $=10.6 \mathrm{~J} / \mathrm{cm}^{2}$

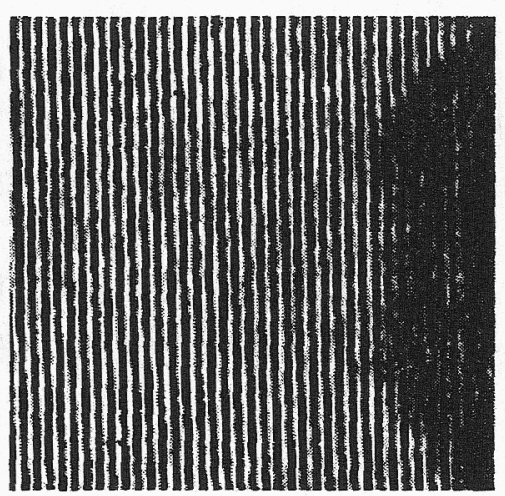

theak $=12.9 \mathrm{~J} / \mathrm{cm}^{2}$

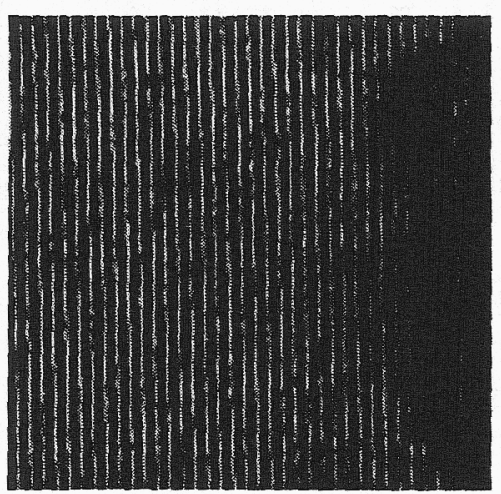

$J_{\text {peak }}=20.1 \mathrm{~J} / \mathrm{cm}^{2}$

Figure 3.3.1.2. Interferograms and shadowgraphs showing LIBA-generated plumes produced by 1.85 ps pulses ablating $.50 \mu \mathrm{m}$ films. Each interferogram was captured 255 ns after the arrival of the ablation pulse. 
In figure 3.3.1.3, interferograms show LIBA-generated plumes produced by ablating .25 $\mu \mathrm{m}$ films with 3.33 ps pulses. Again, we see the "blunting effect". This blunting effect occurs at lower velocities in the 1.85 ps case shown in figure 3.3.1.4. One sees in these interferograms that for a given fluence range and pulsewidth, the $.25 \mu \mathrm{m}$ film ablates at a higher velocity than that of the $0.5 \mu \mathrm{m}$ film.

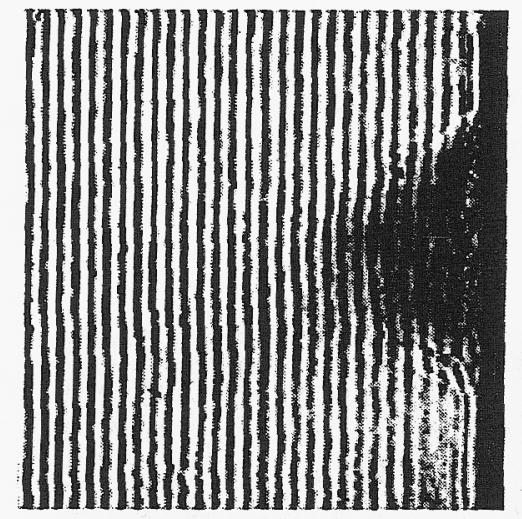

tpeak $=7.07 \mathrm{~J} / \mathrm{cm}^{2}$

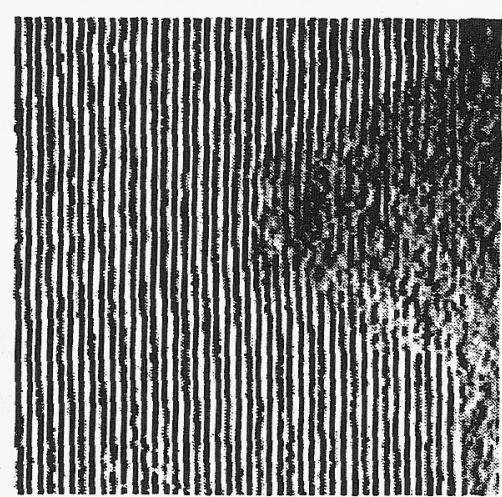

tpeak $=14.2 \mathrm{~J} / \mathrm{cm}^{2}$

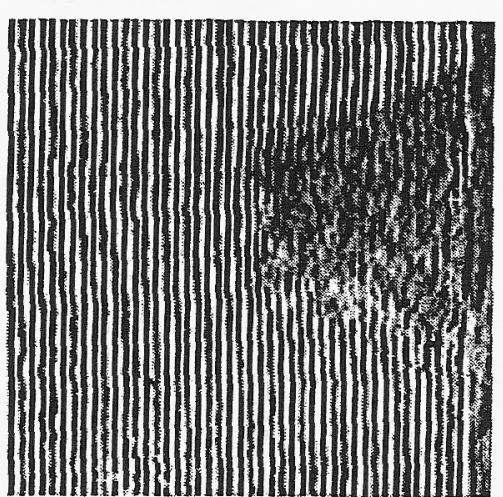

peak $=33.8 \mathrm{~J} / \mathrm{cm}^{2}$

Figure 3.3.1.3. Interferograms and shadowgraphs showing LIBA-generated plumes produced by 3.33 ps pulses ablating $.25 \mu \mathrm{m}$ films. Each interferogram was captured 255 ns after the arrival of ablation pulse.

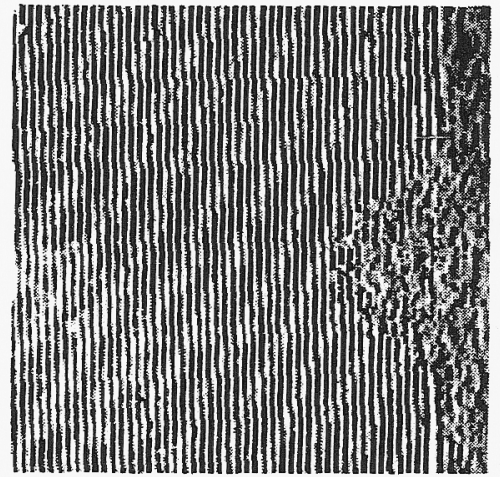

peak $=1.14 \mathrm{~J} / \mathrm{cm}^{2}$

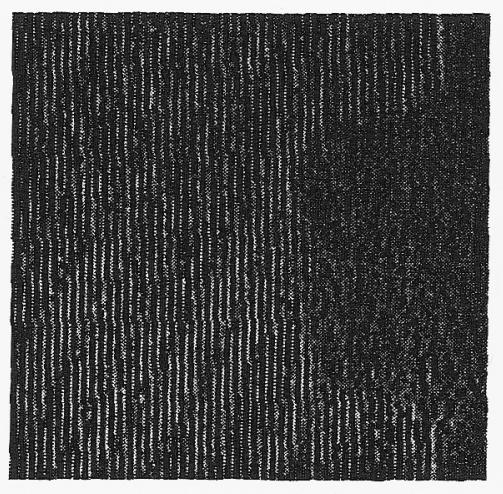

$J_{\text {peak }}=4.61 \mathrm{~J} / \mathrm{cm}^{2}$

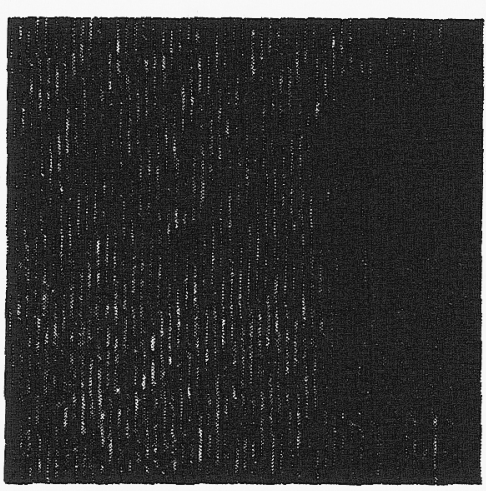

tpeak $=17.1 \mathrm{~J} / \mathrm{cm}^{2}$

Figure 3.3.1.4. Interferograms and shadowgraphs showing LIBA-generated plumes produced by 1.85 ps pulses ablating $.25 \mu \mathrm{m}$ films. Each interferogram was captured 255 ns after the arrival of ablation pulse. 
To study the dependence of edge velocity on incident fluence, we map the spatial beam profile of the incident laser pulse onto the spatial edge velocity profile. Since the probe beam propagates through the plume in the horizontal plane, the plume edge profile shown in the shadowgraphs is a vertical slice of the plume edge at the horizontal center of the plume. I compare this edge to a vertical slice of the 2-D laser beam profile taken from the horizontal center of the beam (see figure 3.3.1.5). This profile slice is first scaled for the focusing effects of the parabola (scaling value ranging from $1.45: 1$ to $1.89: 1$ between front surface profile to back surface profile) and then overlaid on to the plume edge velocity profile. For each point along the vertical axis, there is an edge velocity data point with a corresponding incident front surface fluence value.

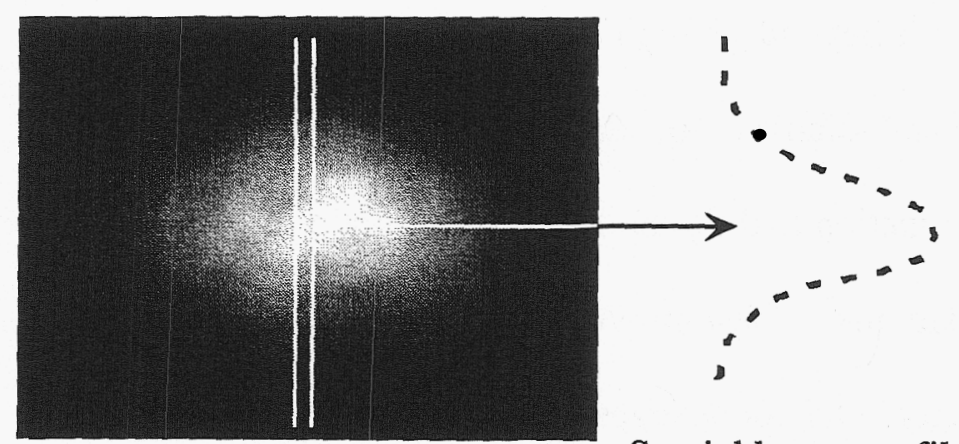

Spatial beam profile

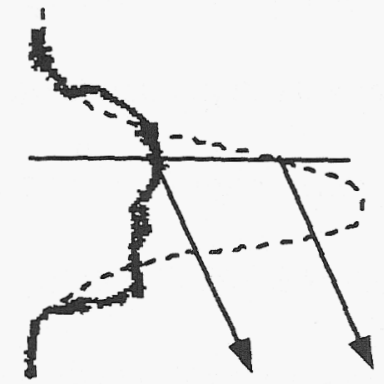

$\begin{array}{lll}\text { Plume } & \text { Velocity } & \text { Fluence } \\ \text { edge } & \text { data } & \text { data } \\ \text { velocity } & \text { point } & \text { point } \\ \text { profile } & & \end{array}$

Figure 3.3.1.5. Diagram of mapping process for comparison of edge velocity to beam profile.

These data points are collected for each plume. The pulse duration, thin film thickness, and incident peak laser fluence are varied during the experiment on a shot-to-shot basis. The data from multiple Al plumes is then collected and plotted together as a set corresponding to a particular film thickness and laser pulse profile. This data is plotted as 
a function of incident front surface fluence. These plots are shown in figures 3.3.1.63.3.1.9.

A cursory examination of the edge velocity plots in Figures 3.3.1.6-3.3.1.9 shows that there are basically three fluence regimes, each with a distinctive edge velocity dependence on fluence. The first fluence regime is characterized by a rapid growth in edge velocity with increasing fluence. In figure 3.3.1.6, this "fast growth" regime extends from $0 \mathrm{~J} / \mathrm{cm}^{2}$ to approximately $5.3 \mathrm{~J} / \mathrm{cm}^{2}$. Extending (in figure 3.3 .1 .6 ) from $5.3 \mathrm{~J} / \mathrm{cm}^{2}$ to approximately $10 \mathrm{~J} / \mathrm{cm}^{2}$, the second fluence regime, the "saturation onset" regime, shows a saturation in the edge velocity growth with increasing fluence. Also, the data typically shows a large amount of scatter in the edge velocity data. With reference to the same figure, the third regime starts at fluences beyond $10 \mathrm{~J} / \mathrm{cm}^{2}$ and is characterized by weak edge velocity dependence on fluence and relatively low data scatter. This is the "asymptotic" regime. We fit a linear function to the data in the "asymptotic" regime, and we fit another linear function to the data in the "fast growth" regime. We can approximate the fluence value at which edge velocity behavior changes in the "saturation onset" regime by determining the intersection of these two lines. 


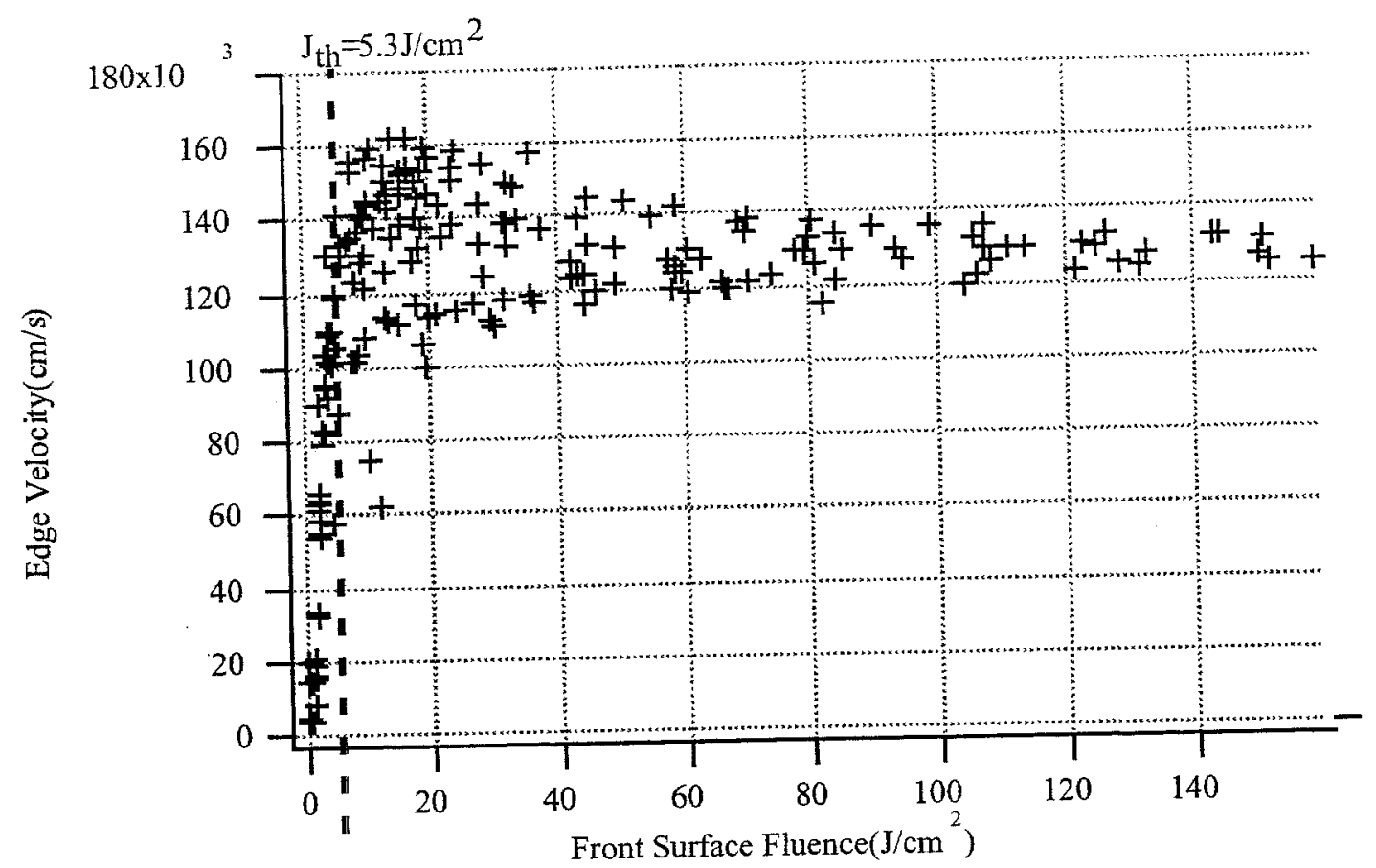

Figure 3.3.1.6. Plot of edge velocity dependence on front surface fluence for $.25 \mu \mathrm{m}$ film ablated by 1.85 ps pulses. 


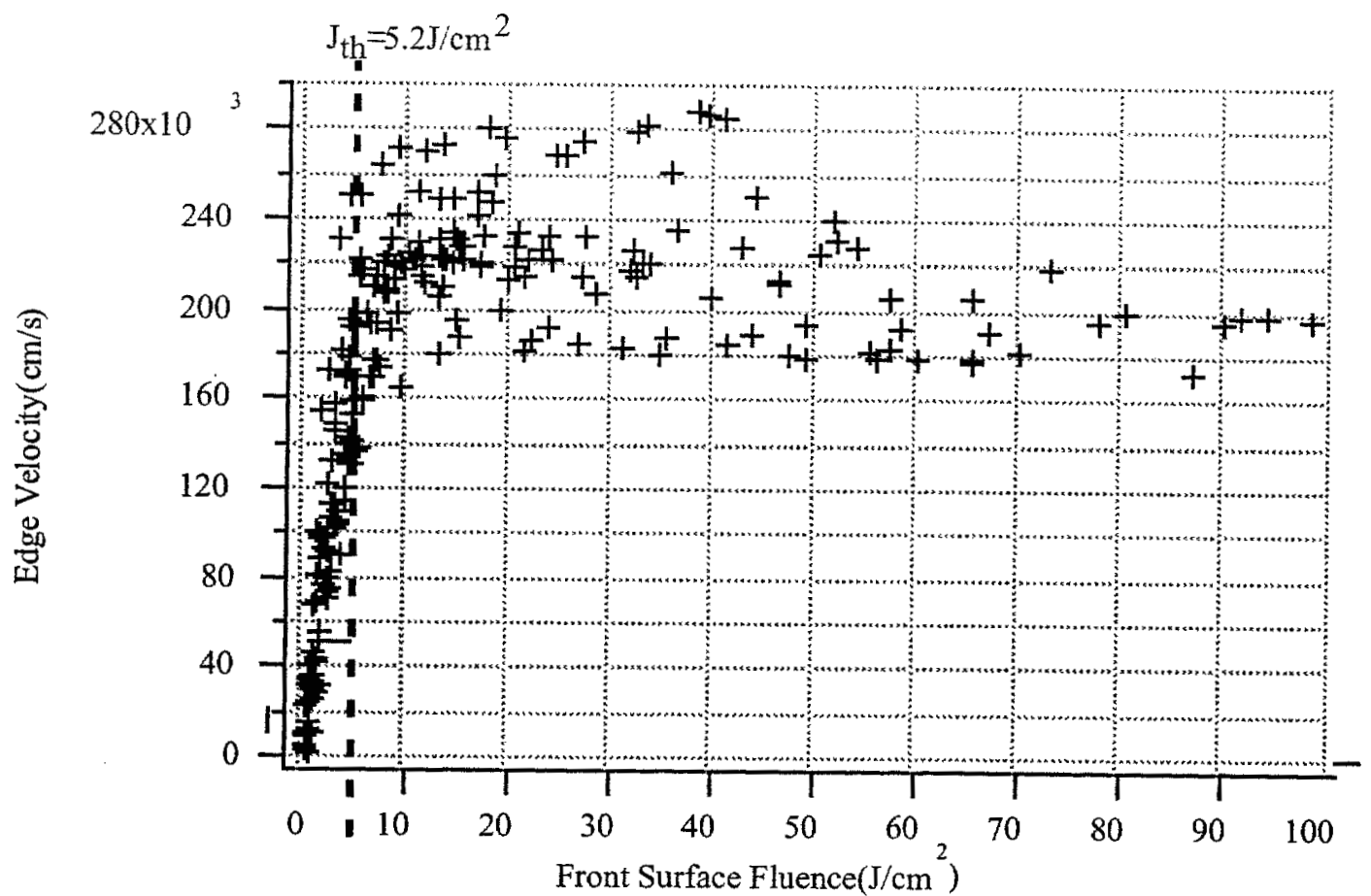

Figure 3.3.1.7. Plot of edge velocity dependence on front surface fluence for $.25 \mu \mathrm{m}$ film ablated by 3.33 ps pulses

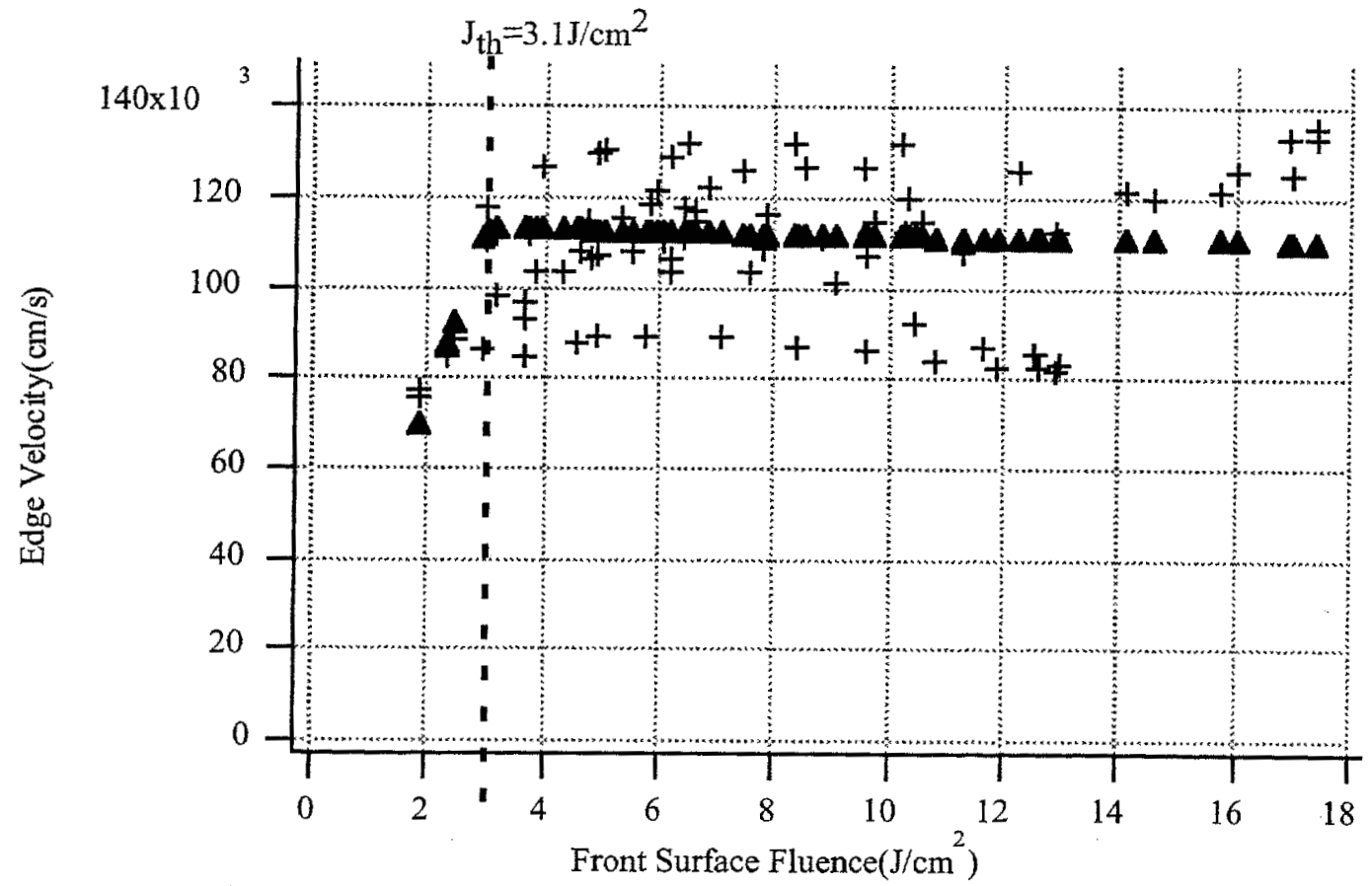

Figure 3.3.1.8. Plot of edge velocity dependence on front surface fluence for $.50 \mu \mathrm{m}$ film ablated by $1.85 \mathrm{ps}$ pulses 


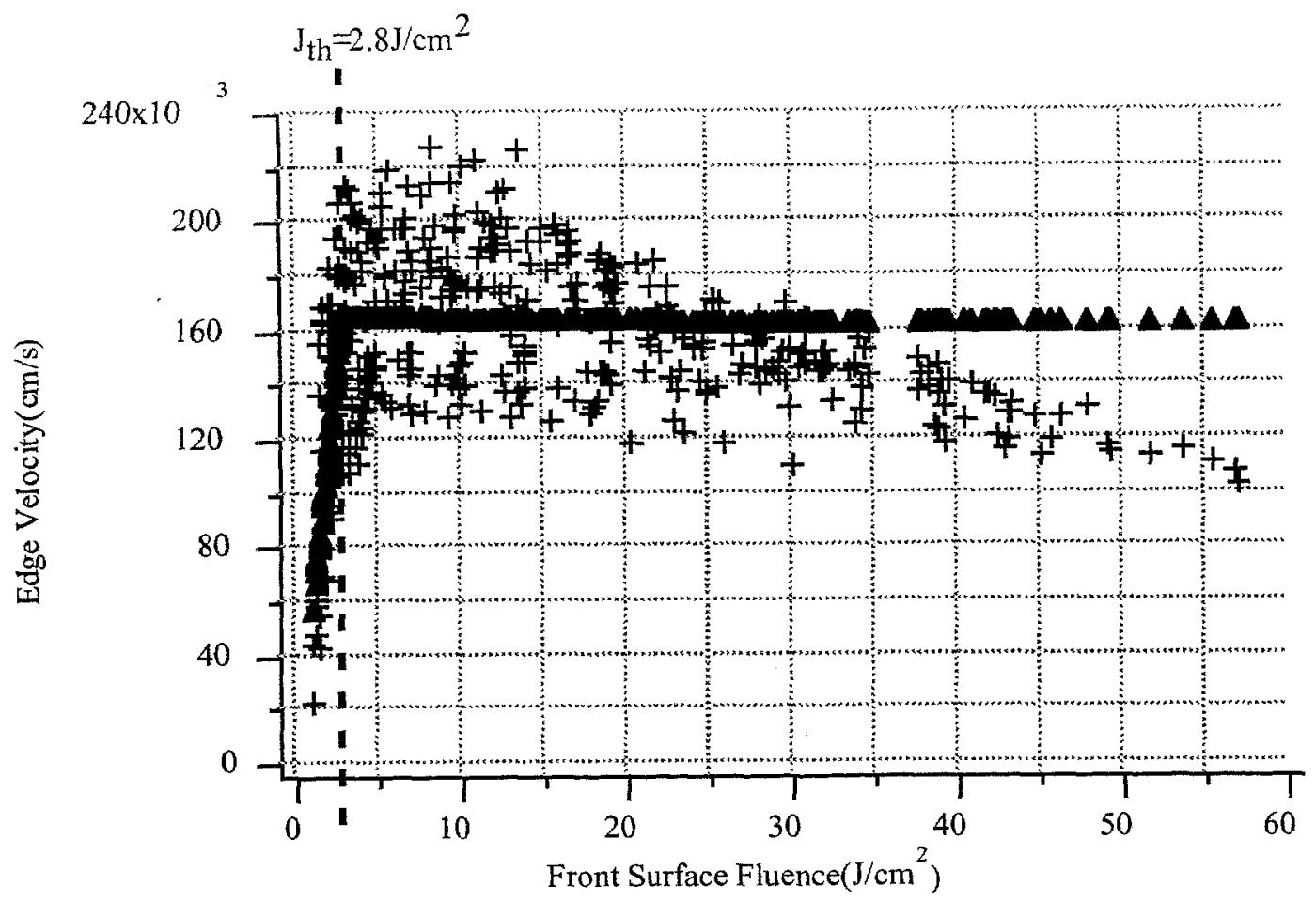

Figure 3.3.1.9. Plot of edge velocity dependence on front surface fluence for $.50 \mu \mathrm{m}$ film ablated by 3.33 ps pulses

This is defined as the transition fluence, $\mathrm{J}_{\mathrm{th}}$, and a chart of these fluence values for particular sets of experimental parameters is shown below.

\begin{tabular}{|c|c|c|}
\hline $\begin{array}{c}\text { Laser Pulse } \\
\text { Duration }\end{array}$ & $\begin{array}{c}\text { Aluminum } \\
\text { Film } \\
\text { Thickness }\end{array}$ & $\begin{array}{c}\text { Transition } \\
\text { Fluence, } \\
\mathrm{J}_{\mathrm{th}}\left(\mathrm{J} / \mathrm{cm}^{2}\right)\end{array}$ \\
\hline $3.33 \mathrm{ps}$ & $.25 \mu \mathrm{m}$ & 5.2 \\
\hline $3.33 \mathrm{ps}$ & $.5 \mu \mathrm{m}$ & 2.8 \\
\hline $1.85 \mathrm{ps}$ & $.25 \mu \mathrm{m}$ & 5.3 \\
\hline $1.85 \mathrm{ps}$ & $.5 \mu \mathrm{m}$ & 3.1 \\
\hline
\end{tabular}

Table 3.3.1.1. Table of threshold fluence values as a function of film thickness and laser pulse duration.

Examination of the edge velocity in the asymptotic region ( see figures 3.3.1.6-3.3.1.9) reveals several interesting features. A fluence range corresponding to the asymptotic 
region is selected for comparison. Dividing the edge velocity of the $.25 \mu \mathrm{m}, 1.85$ ps case by the edge velocity of the $.25 \mu \mathrm{m}, 3.33$ ps case and averaging this ratio over the range from $10 \mathrm{~J} / \mathrm{cm}^{2}$ to $22 \mathrm{~J} / \mathrm{cm}^{2}$ produces an average edge velocity ratio, $<\mathrm{u}_{1}>$, of $0.588 \pm$ 0.065. In this case, as well as those below, the averaging range is chosen to produce the most representative average value. This range is also limited by the maximum front surface fluence used in the experiment. Similarly, dividing the edge velocity of the .50 $\mu \mathrm{m}, 1.85$ ps case by the edge velocity of the $.50 \mu \mathrm{m}, 3.33$ ps case and averaging this ratio over the range from $10 \mathrm{~J} / \mathrm{cm}^{2}$ to $22 \mathrm{~J} / \mathrm{cm}^{2}$ produces an average edge velocity ratio, $<\mathrm{u}_{2}>$, of $0.649 \pm 0.098$. It is interesting to note that $\left\langle\mathrm{u}_{1}>\right.$ and $<\mathrm{u}_{2}>$ have values that are statistically indistinguishable. This indicates that the laser/aluminum absorption mechanism is not a strong function of film thickness. We may also look at the effect of film thickness on edge velocity. Dividing the edge velocity of the $.50 \mu \mathrm{m}, 3.33$ ps case by the edge velocity of the $.25 \mu \mathrm{m}, 3.33$ ps case and averaging this ratio over the range from $10 \mathrm{~J} / \mathrm{cm}^{2}$ to $57 \mathrm{~J} / \mathrm{cm}^{2}$ produces an average edge velocity ratio, $\left\langle\mathrm{u}_{3}>\right.$, of $0.644 \pm 0.107$. Dividing the edge velocity of the $.50 \mu \mathrm{m}, 1.85$ ps case by the edge velocity of the $.25 \mu \mathrm{m}$, $1.85 \mathrm{ps}$ case and averaging this ratio over the range from $10 \mathrm{~J} / \mathrm{cm}^{2}$ to $17 \mathrm{~J} / \mathrm{cm}^{2}$ produces an average edge velocity ratio, $\left\langle u_{4}>\right.$, of $0.864 \pm 0.113$. Both $\left\langle u_{3}>\right.$ and $<u_{4}>$ have values near $\sqrt{2} / 2$, the value expected from a simple scaling of velocity with mass, assuming a constant amount of kinetic energy in the plume.

\subsubsection{Edge Velocity Behavior at Low Laser Fluence}

Observing the behavior of edge velocity at low fluence allows the study of several LIBA features in the "fast growth" regime. In figure 3.3.2.1, the edge velocity data is 
plotted as a function of front surface fluence. This data closely resembles a square root function with an offset, and we fit a curve to the data using the following functional form:

$$
v_{e d g e}=C \times \sqrt{J-J_{a b}},
$$

where $\mathrm{C}$ is a constant, $\mathrm{J}$ is the incident front surface fluence, and $\mathrm{J}_{\mathrm{ab}}$ is a fluence threshold above which ablation can occur. In figure 3.3.3.1, the best fit to the functional form for the $0.25 \mu \mathrm{m}, 3.33$ ps case is with the values $C=75792$ and $J_{a b}=1.2 \mathrm{~J} / \mathrm{cm}^{2}$. Similarly, in figure 3.3 .2 .2 , the best fit values for the $0.25 \mu \mathrm{m}, 1.85$ ps case are $C=83096$ and $J_{a b}=0.7$ $\mathrm{J} / \mathrm{cm}^{2}$, and in figure 3.3.2.3, the best fit values for the $0.5 \mu \mathrm{m}, 3.33 \mathrm{ps}$ are $\mathrm{C}=71882$ and $\mathrm{J}_{\mathrm{ab}}=0.68 \mathrm{~J} / \mathrm{cm}^{2}$. Since a square root functional form can be fit to the edge velocity in this low fluence regime, the Laser Energy Transfer Efficiency (LETE), the fraction of incident laser energy which is converted to kinetic energy, has approximately a constant value. Also, the existence of a fluence threshold in both the raw data and in the curve fit supports the idea that, below a certain fluence value, the $\mathrm{Al}$ thin film can absorb laser energy without being ablated from the substrate. However, the variation in fluence threshold shown above is not explained by the difference in pulsewidth and film thickness. Instead, it suggests that, at very low fluences near $0 \mathrm{~J} / \mathrm{cm}^{2}$, the edge velocity does not follow the square root functional form. In fact, a simple look at figure 3.3.2.1 shows that non-zero edge velocity values are shown at front surface fluences below 1 $\mathrm{J} / \mathrm{cm}^{2}$. Based on these observations, the estimates produced by this simple curve fit can only be seen as coarse estimates of the actual fluence threshold. 


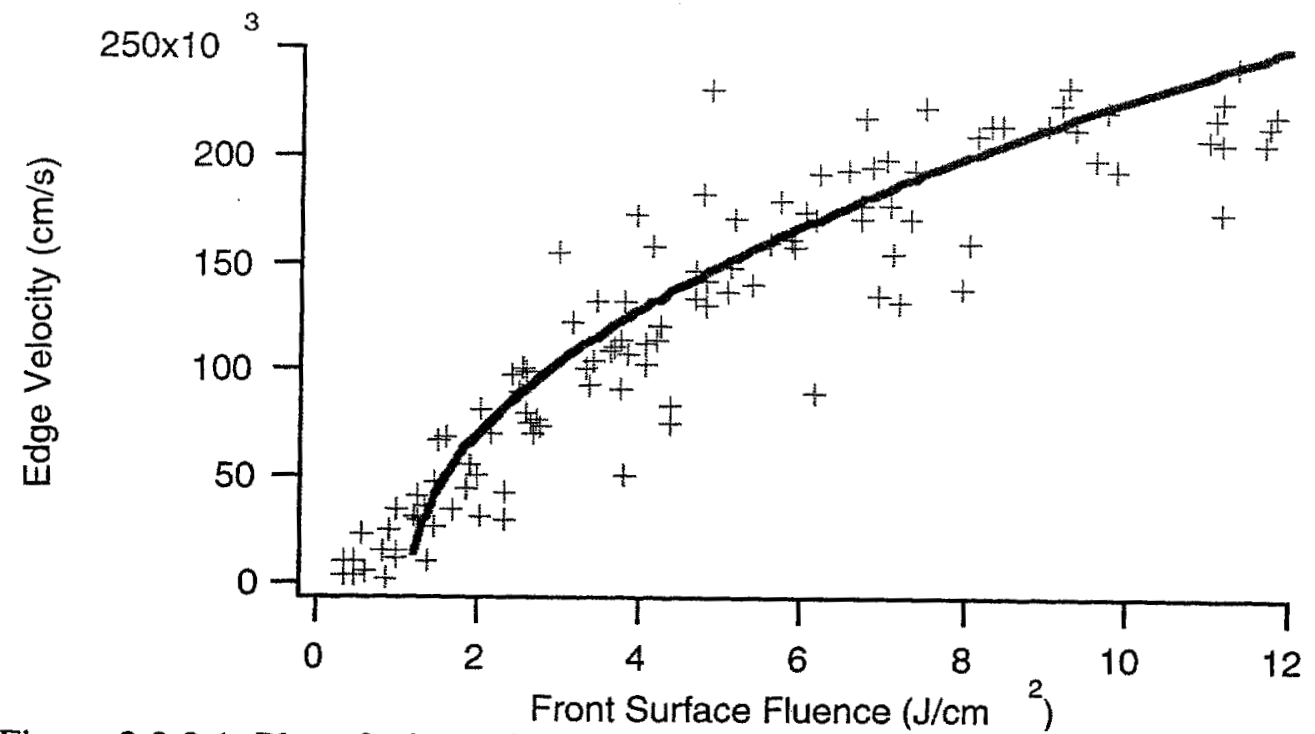

Figure 3.3.2.1. Plot of edge velocity as a function of front surface fluence for plumes produced by $0.25 \mu \mathrm{m}$ films ablated by 3.33 ps laser pulses. The solid line is a curve fit to the data. 


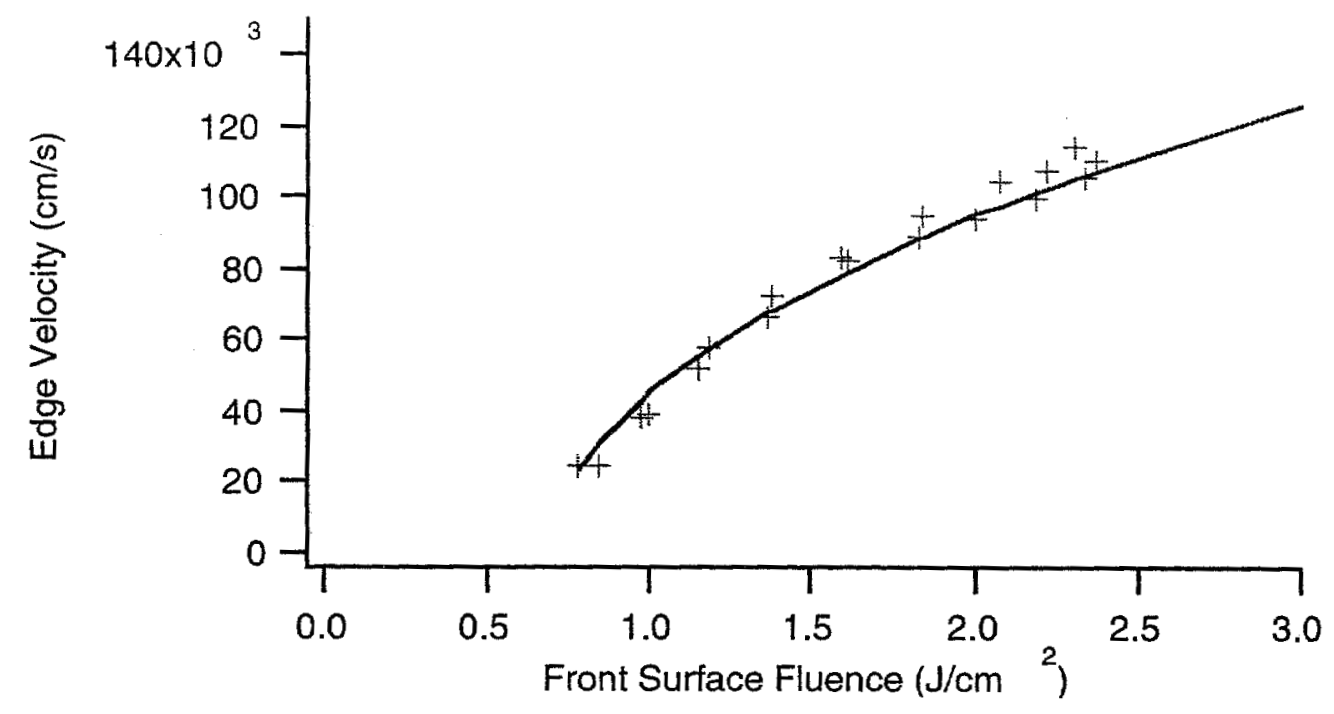

Figure 3.3.2.2. Plot of edge velocity as a function of front surface fluence for plumes produced by $0.25 \mu \mathrm{m}$ films ablated by 1.85 ps laser pulses. The solid line is a curve fit to

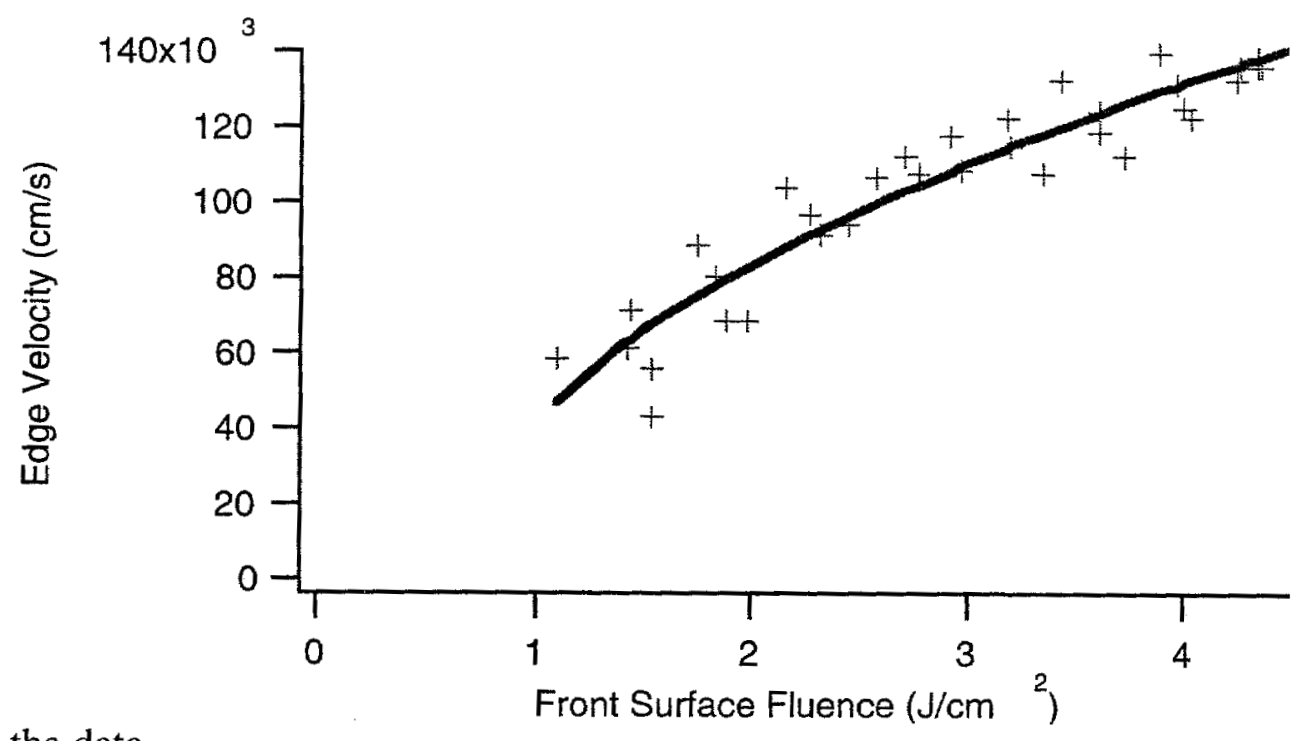

the data.

Figure 3.3.2.3. Plot of edge velocity as a function of front surface fluence for plumes produced by $0.50 \mu \mathrm{m}$ films ablated by 3.33 ps laser pulses. The solid line is a curve fit to the data. 


\subsubsection{Observations of Plume Edge Velocity at Multiple Delay Times}

Studying the plume edge velocity at multiple shadowgraph time delays allows the examination of transverse plume divergence. Plume divergence is directly related to plume temperature and measuring the divergence allows us to estimate this temperature. In figure 3.3.3.1(A) and (B), the plume is shown at two different delay times, $255 \mathrm{~ns}$ and $320 \mathrm{~ns}$. There is minimal transverse expansion in the plume at $320 \mathrm{~ns}$ relative to the plume at $255 \mathrm{~ns}$. Using the simple relation, $\mathrm{mv}^{2} / 2=3 \mathrm{kT} / 2$, we find that even the relatively low average temperature of $0.5 \mathrm{eV}$ would lead to a transverse expansion velocity on order $2 \times 10^{5} \mathrm{~cm} / \mathrm{s}$. This is far in excess of any observed expansion velocity, and therefore the actual average plume temperature must be much less than $0.5 \mathrm{eV}$. Additionally, figure 3.3.3.2 shows the edge velocity data from figure 3.3.3.1(A) and 3.3.3.1 (B) plotted together as a function of front surface fluence. The lack of a velocity offset in the $320 \mathrm{~ns}$ data shows there is no noticeable acceleration occurring after 255 ns. Figure 3.3.3.3 shows the full data set for the $0.25 \mu \mathrm{m}, 1.85 \mathrm{ps}$ case, and again, no acceleration is observed. The lack of acceleration indicates that plume temperature has dropped to a level such that hydrodynamic plume expansion would be insignificant, and the plume can be considered to be a very low temperature distribution of $\mathrm{Al}$ atoms. 

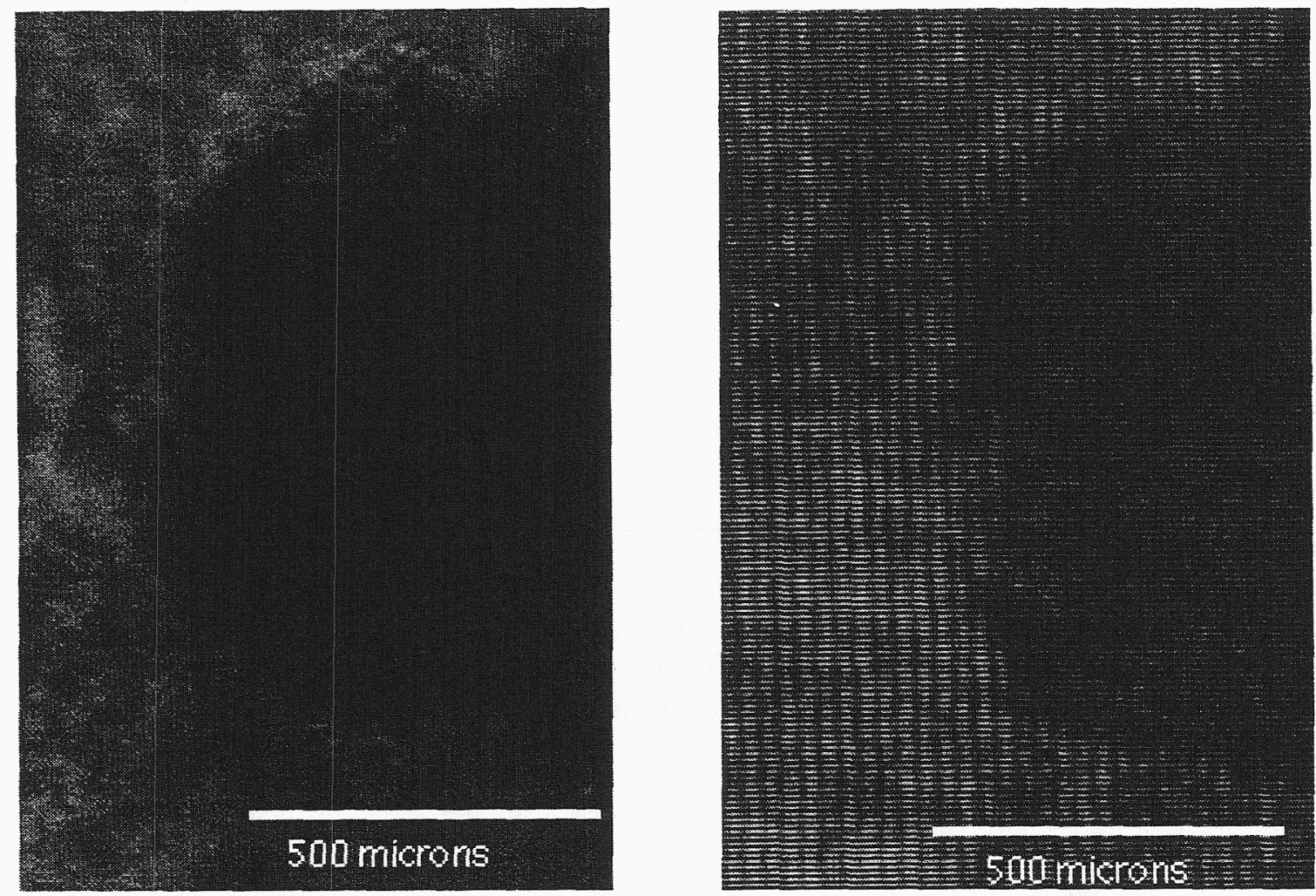

(A)

(B)

Figure 3.3.3.1. Images of a metal plume formed by a $0.25 \mu \mathrm{m}$ thin film ablated by a 1.85 ps pulse. Image (A) (Peak Front Surface Fluence $=4.61 \mathrm{~J} / \mathrm{cm}^{2}$ ) was taken $320 \mathrm{~ns}$ after the arrival of the ablation pulse, and image (B) was taken $255 \mathrm{~ns}$ after the arrival of the ablation pulse. Images were obtained with a $527 \mathrm{~nm}$ probe pulse. 


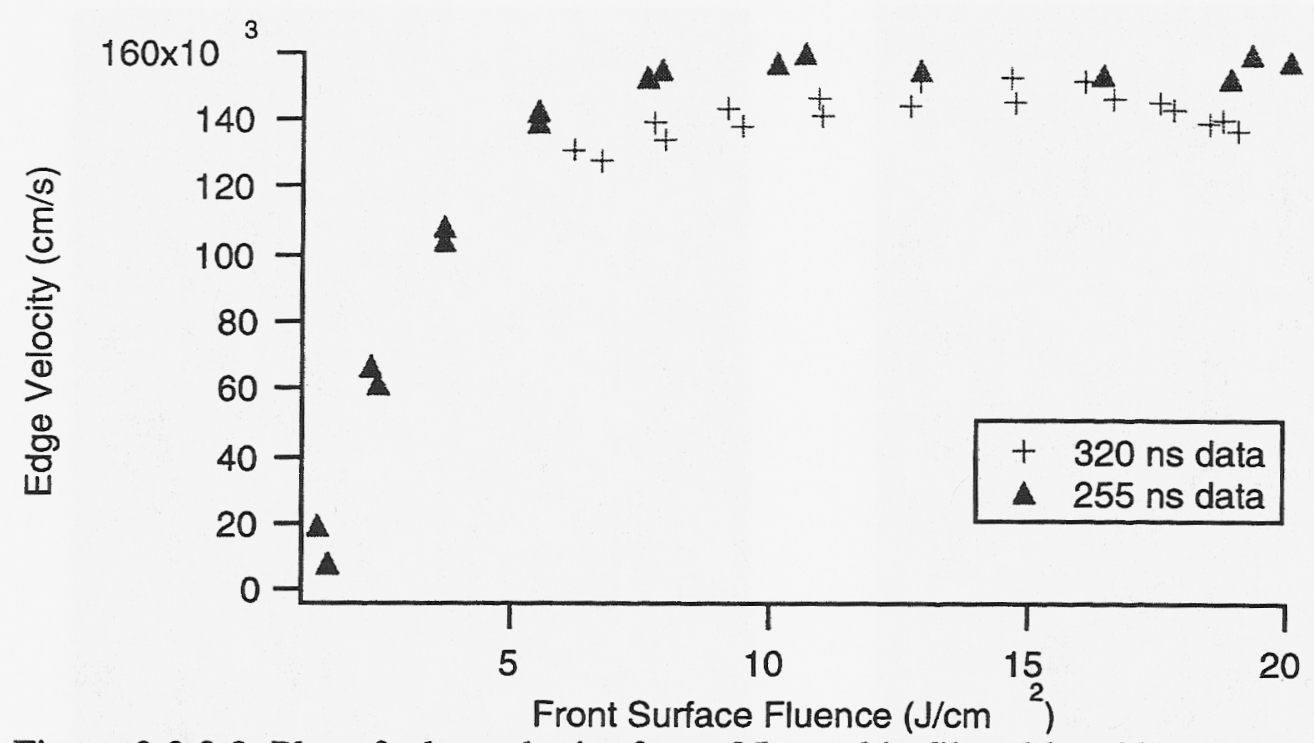

Figure 3.3.3.2. Plot of edge velocity for a $.25 \mu \mathrm{m}$ thin film ablated by a $1.85 \mathrm{ps}$ laser pulse. The edge velocities show negligible acceleration in the time period between $255 \mathrm{~ns}$ and 320 ns.

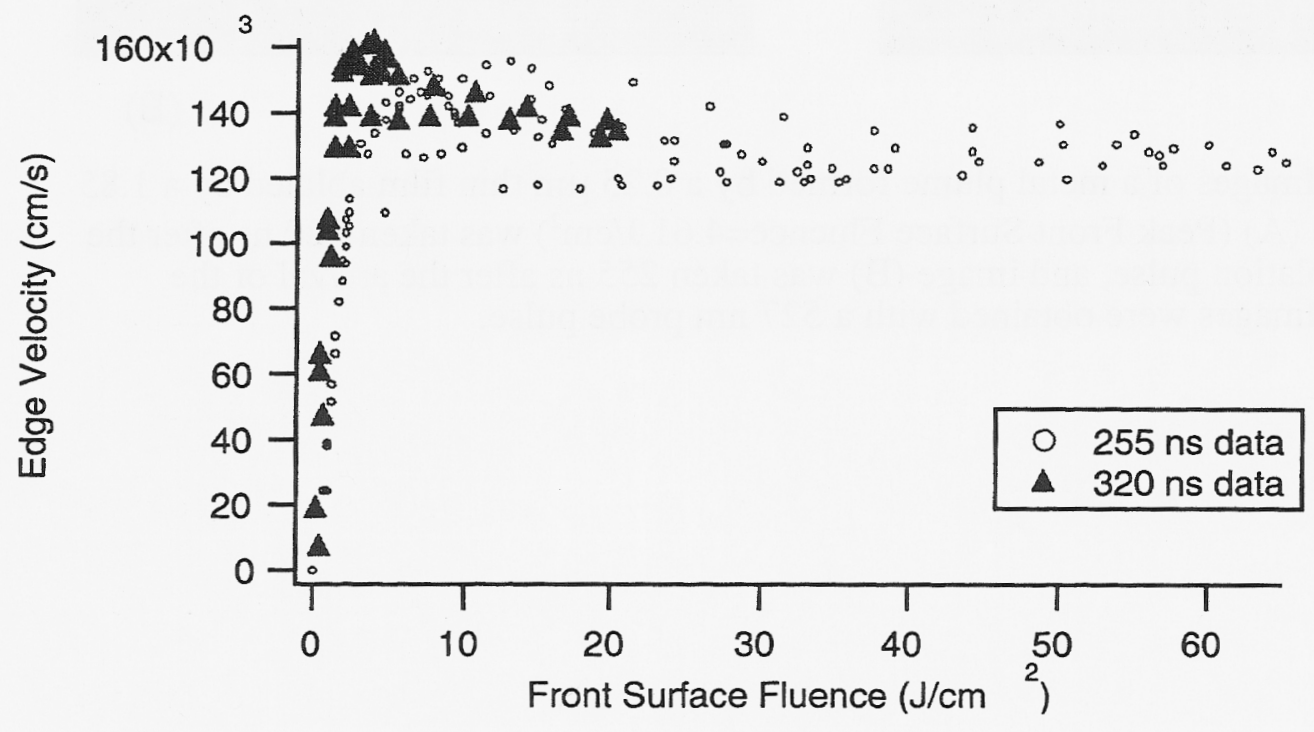

Figure 3.3.3.3. Plot of edge velocity data set for a $.25 \mu \mathrm{m}$ thin film ablated by a $1.85 \mathrm{ps}$ laser pulse. The edge velocities show negligible acceleration in the time period between $255 \mathrm{~ns}$ and $320 \mathrm{~ns}$. 


\subsection{Interferogram Observations}

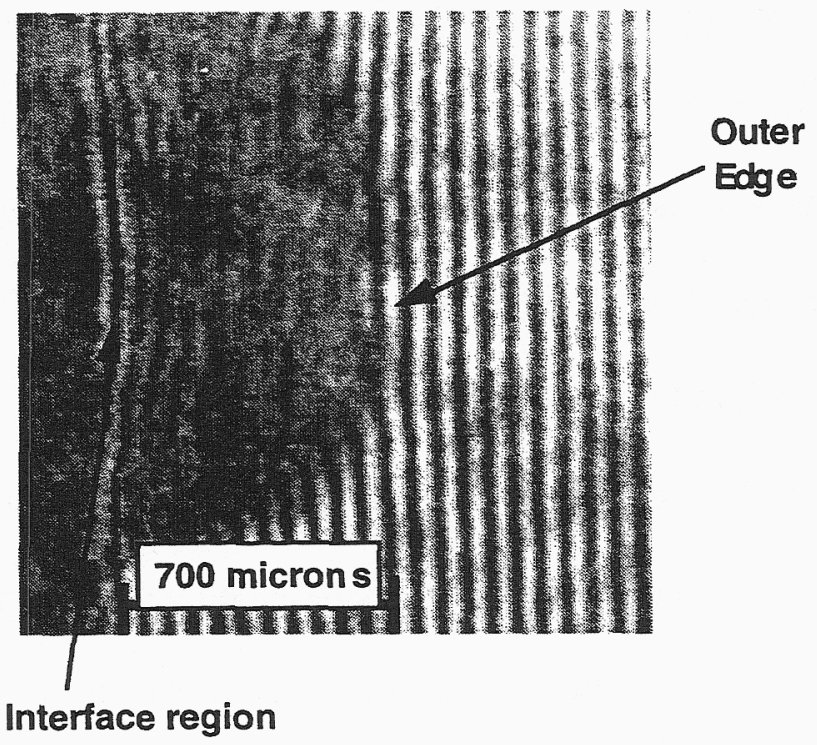

Figure 3.4.1. An interferogram $(527 \mathrm{~nm})$ of a $.25 \mu \mathrm{m}$ film ablated by a 3.33 ps pulse $(11.1$ $\mathrm{J} / \mathrm{cm}^{2}$ peak front surface fluence). The interferogram was taken with a $255 \mathrm{~ns}$ delay after the ablation event.

Using the self-referencing interferometer to produce $1 \omega$ and $2 \omega$ interferograms of the ablated plume, several interesting plume features are observed. The interferogram of a $0.25 \mu \mathrm{m}$ Al film ablated by a 3.33 ps pulse is shown in figure 3.4.1. Included in the interferogram is the shadow of the ablated $\mathrm{Al}$ plume. In the region near the outer edge of the ablated plume, the fringes are seen to shift towards the substrate/film interface. The fringes near the interface, however, are seen to shift away from the interface and towards the outer edge of the Al plume. Since the electron-induced phase shift is of opposite sign to that of the neutral atom phase shift, these observations suggest that neutral atoms are dominating the total phase shift at one location while the electrons are dominating the phase shift at the other location. Since, in early time, laser heating and ionization is limited to the region of the Al thin film in close proximity (tens of nanometers) to the 
interface, one would conclude that, the region of high electron density would be near the interface. The outer edge of the film (away from the interface) does not experience direct laser heating and therefore has a lower electron density than the interface region. One would then expect the phase shift at the outer edge to be dominated by neutral atoms. Using the interferograms and assuming azimuthal symmetry, one can estimate the interaction length of the plume at the outer edge to be $875 \mu \mathrm{m}$ and the interaction length at the interface to be $1575 \mu \mathrm{m}$. One then finds the neutral atom density at the outer edge to be of order $10^{18} \mathrm{~cm}^{-3}$ and the electron density at the interface to be of order $10^{15} \mathrm{~cm}^{-3}$. A simple calculation of the average neutral density based on the expansion of the film from $.25 \mu \mathrm{m}$ to $600 \mu \mathrm{m}$, gives a value near $10^{19} \mathrm{~cm}^{-3}$, so these estimates are reasonable.

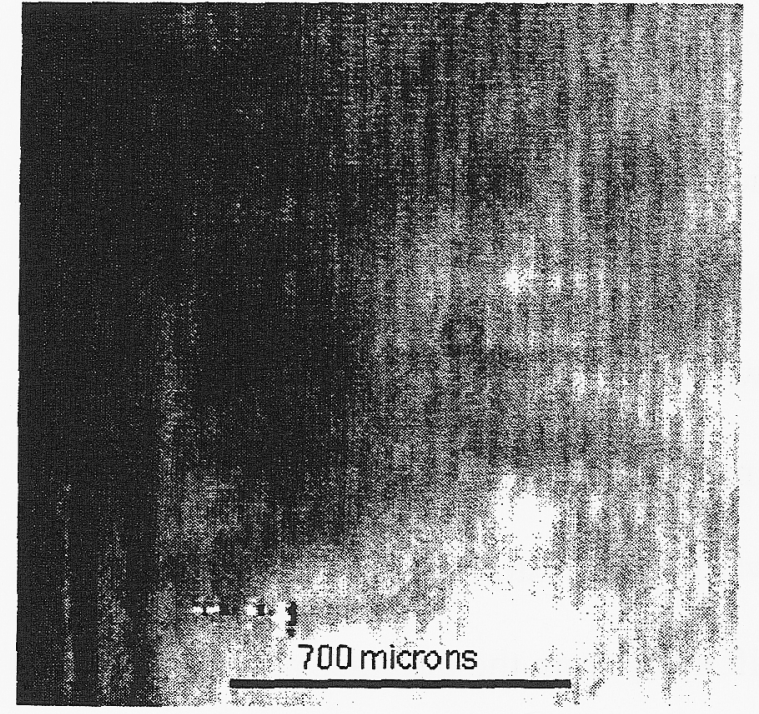

(A)

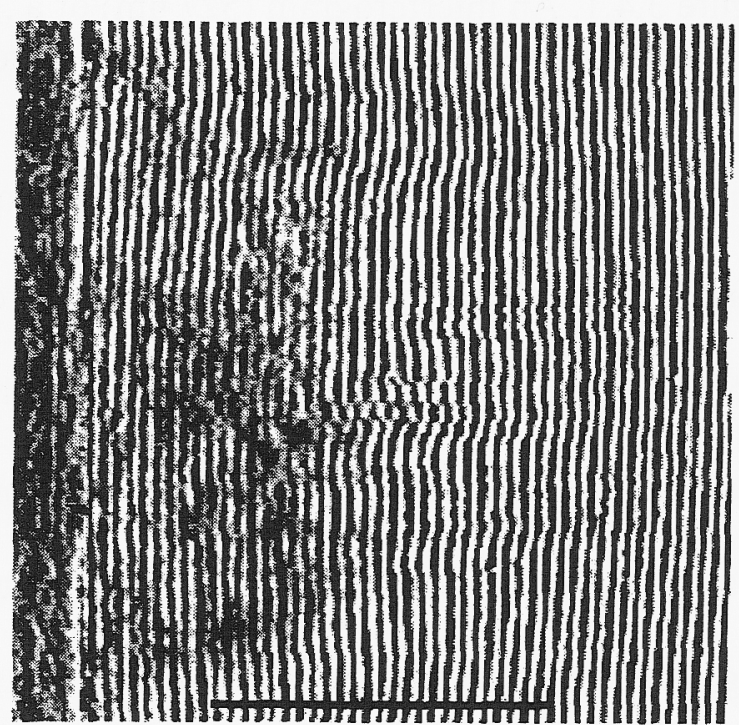

(B)

700 microns

Figure 3.4.2. Interferograms of a $0.1 \mu \mathrm{m} \mathrm{Al} \mathrm{film} \mathrm{ablated} \mathrm{by} 3.33 \mathrm{ps}$ ablation pulse (13.5 $\mathrm{J} / \mathrm{cm}^{2}$ peak front surface fluence). Image (A) is taken with a $1053 \mathrm{~nm}$ probe beam, while image (B) is taken with a $527 \mathrm{~nm}$ probe beam. Both images were captured $255 \mathrm{~ns}$ after arrival of the ablation pulse.

In comparison with figure 3.4.1, the interferogram shown in figure 3.4.2(A) and 3.4.2(B) shows $0.1 \mu \mathrm{m} \mathrm{Al} \mathrm{film} \mathrm{ablated} \mathrm{by} \mathrm{3.33ps} \mathrm{pulse.} \mathrm{Unlike} \mathrm{figure} \mathrm{3.4.1,} \mathrm{the} \mathrm{plumes}$ 
shown here do not have a well-defined plume shape. Also, the phase shift distribution shown in figure $3.4 .2(\mathrm{~B})$ is asymmetric with respect to the center of the plume. The plume shadows shown in figures 3.4 .2 (A) and (B) also show this asymmetric behavior. This contrasts sharply with the plume shown in figure 3.4.1, and it suggests that the $0.1 \mu \mathrm{m}$ plume may be at a higher temperature than the $0.25 \mu \mathrm{m}$ plume, even at the outer edges of the plume. Using the two interferograms shown in figure 3.4.2(A) and 3.4.2(B),

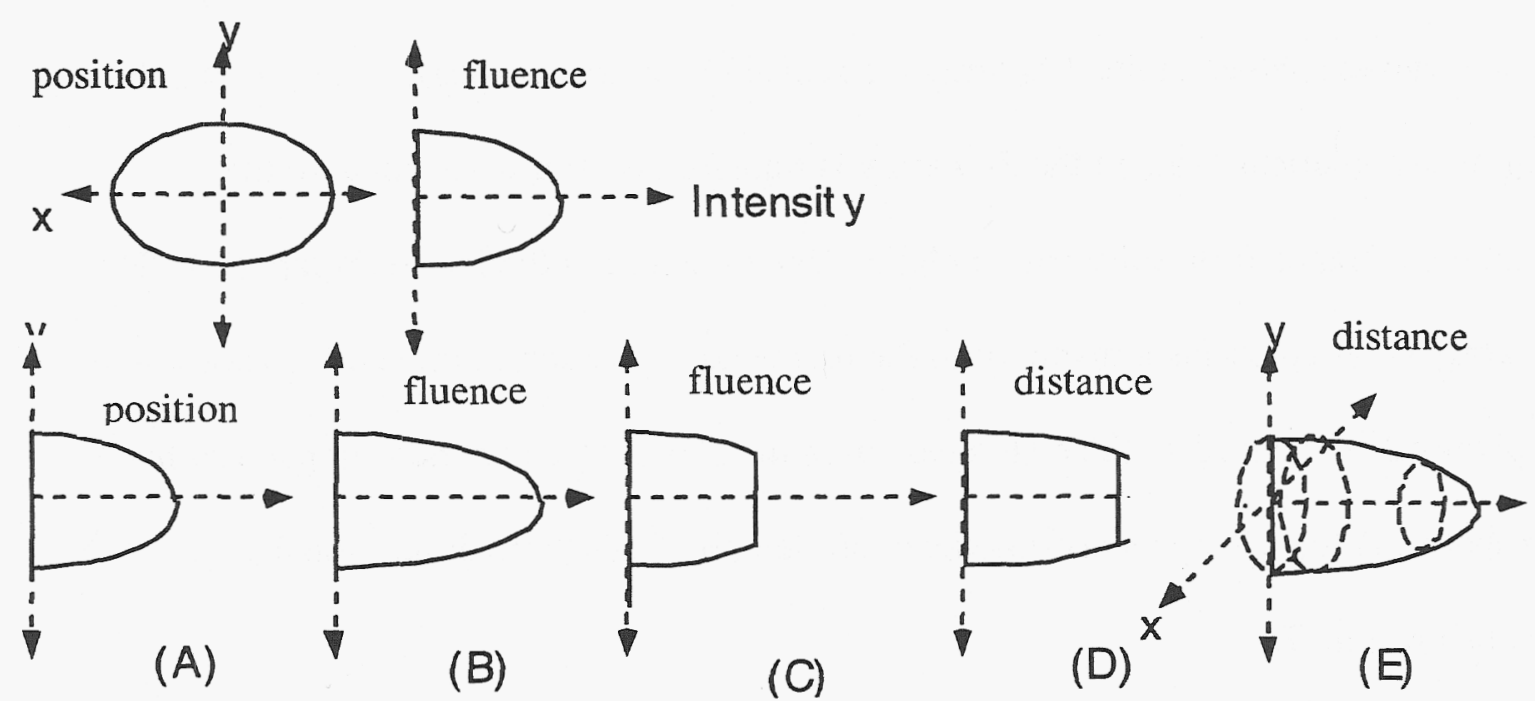
we calculate the neutral and electron density profiles (see figure 3.4.4(A) and 3.4.4(B))

Figure 3.4.3. Diagram describing how a 2-D spatial laser intensity profile is transformed into an approximate plume shape.

along the center of the plume. In these calculations, the interaction length, $T$, was needed to solve for the neutral and electron density. Since direct measurement of $\mathrm{T}$ at each point in the plume is difficult, $\mathrm{T}$ is estimated using a simple model (see figure 3.4.3).

The 2-D spatial spot profile of the laser pulse is digitized into a two-dimensional array of pixel values (A). Here, the x-y plane represents the spatial spot profile dimensions. Each numerical value is then scaled by a constant $C_{1}$ such that the peak of 
the 2-D profile has a value equal to the peak incident fluence of the ablation pulse at the substrate front surface(B). At this point, the 2-D array represents the fluence distribution of the incident laser pulse. Using the substrate-damage model to be discussed in chapter 4 , each value in the 2-D array is then adjusted such that each pixel is now equal to the transmitted fluence at the substrate/film interface (C). This results in a "blunted" 2-D array which represents an estimate of the transmitted spatial laser spot profile. At this point, the edge velocity of the plume is assumed linearly dependent on transmitted fluence, and the values in the 2-D array are scaled by another constant, $C_{2}$, such that the maximum numerical value in the 2-D array is transformed to a value equal to the maximum distance from substrate to plume edge observed in the interferogram (D). The 2-D array now represents a mapping of the plume edge position in three dimensions, with the pixel value equating to the edge position in the $\mathrm{z}$ axis. One can then simple use this 2$\mathrm{D}$ array to calculate $\mathrm{T}$ by measuring the chord length of this three dimensional edge model shown in $(E)$.

Note (see figure 3.4.4(A) and 3.4.4(B)), in the region between 500 and 1000 microns, the relative electron and neutral atom density distributions are similar. This indicates that, unlike the $.25 \mu \mathrm{m}$ case, the level of ionization in this region of the plume is approximately constant (see figure $3.4 .4(\mathrm{C})$ ), which suggests an approximately constant temperature throughout this plume region. While laser absorption occurs in the first few skin depths (within tens of microns away from the interface), the timescale for ablation may allow for heat conduction through the entire thickness of the film. Therefore a constant level of ionization is more likely for thinner films. In this region, the level of 
ionization is of order 0.001 . This level of ionization confirms a very low temperature $(\mathrm{T}<<1 \mathrm{eV})$ at the delay time of the interferogram $(255 \mathrm{~ns})$.

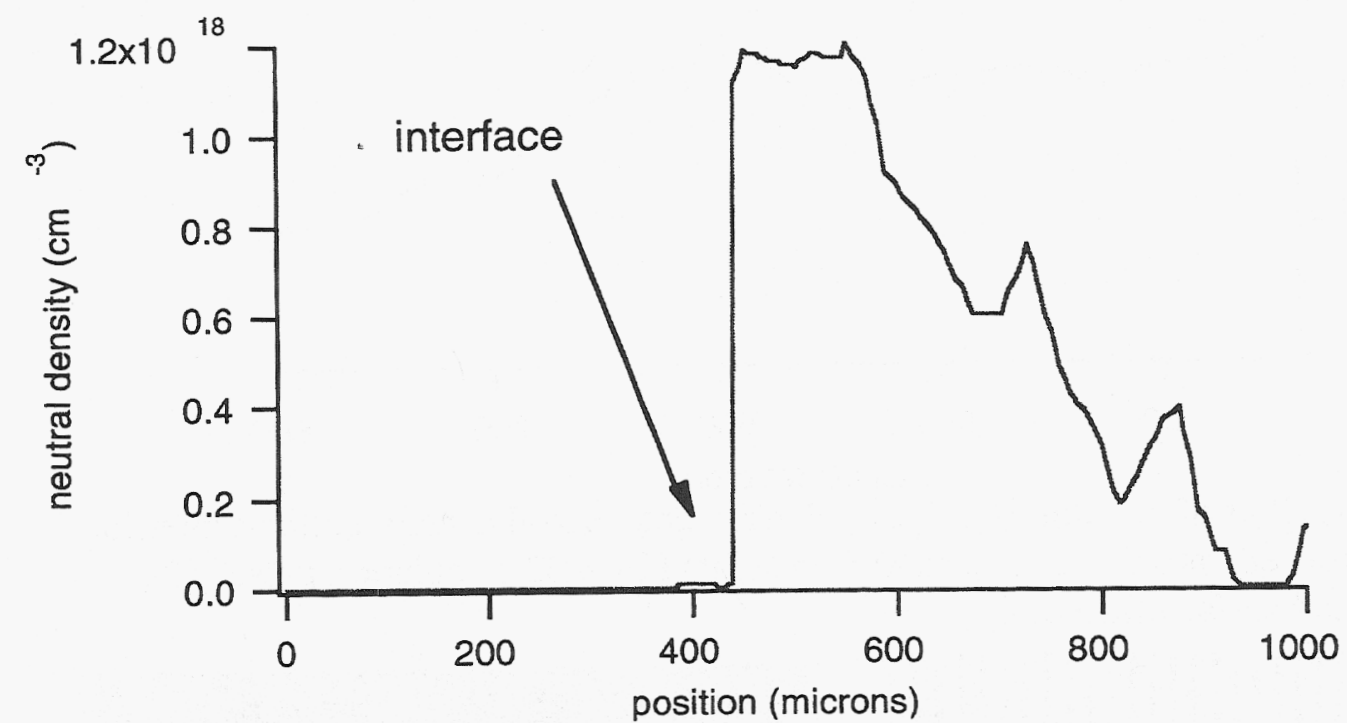

Figure 3.4.4(A). Plot of neutral density on the centerline of a $0.1 \mu \mathrm{m}$ Al film ablated by $3.33 \mathrm{ps}$ ablation pulse $\left(13.5 \mathrm{~J} / \mathrm{cm}^{2}\right.$ peak front surface fluence).

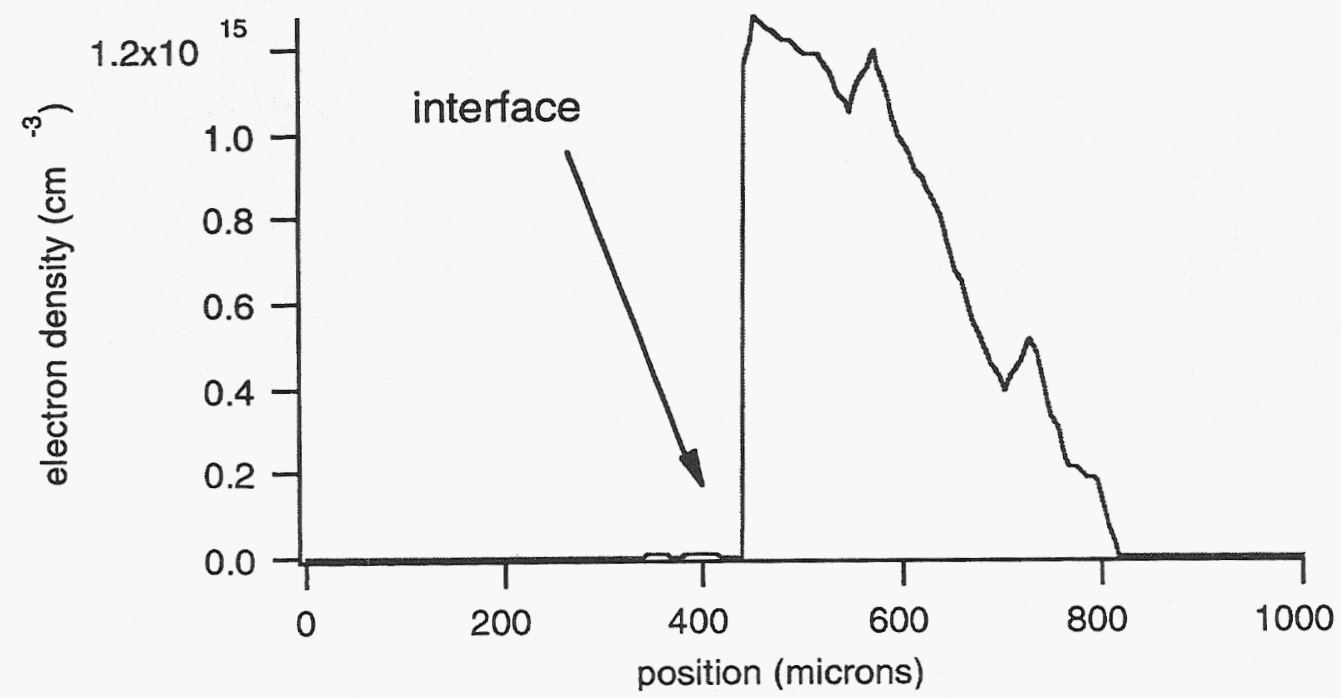

Figure 3.4.4(B). Plot of electron density on the centerline of an $\mathrm{Al}$ plume generated by a $0.1 \mu \mathrm{m}$ Al film ablated by $3.33 \mathrm{ps}$ ablation pulse $\left(13.5 \mathrm{~J} / \mathrm{cm}^{2}\right.$ peak front surface fluence). 


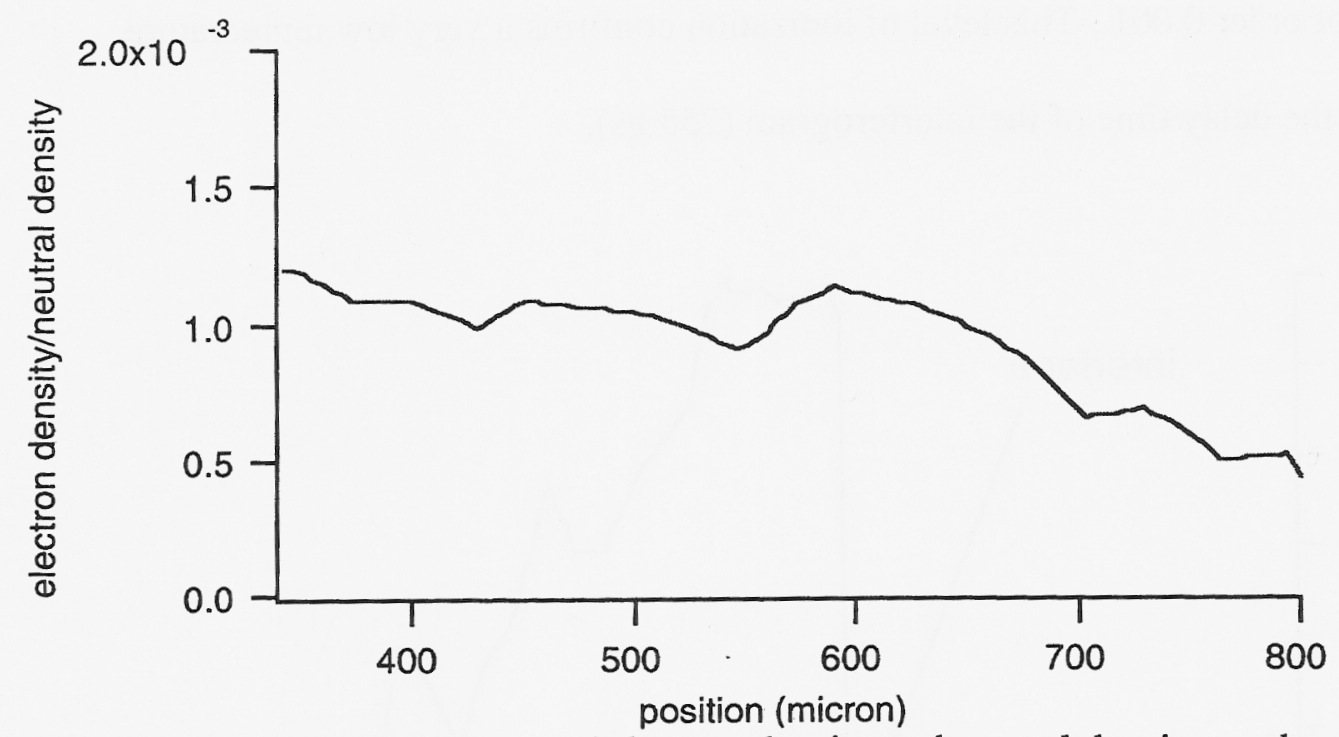

Figure 3.4.4(C) Plot of the ratio of electron density and neutral density on the centerline of an $\mathrm{Al}$ plume generated by a $0.1 \mu \mathrm{m} \mathrm{Al}$ film ablated by $3.33 \mathrm{ps}$ ablation pulse (13.5 $\mathrm{J} / \mathrm{cm}^{2}$ peak front surface fluence).

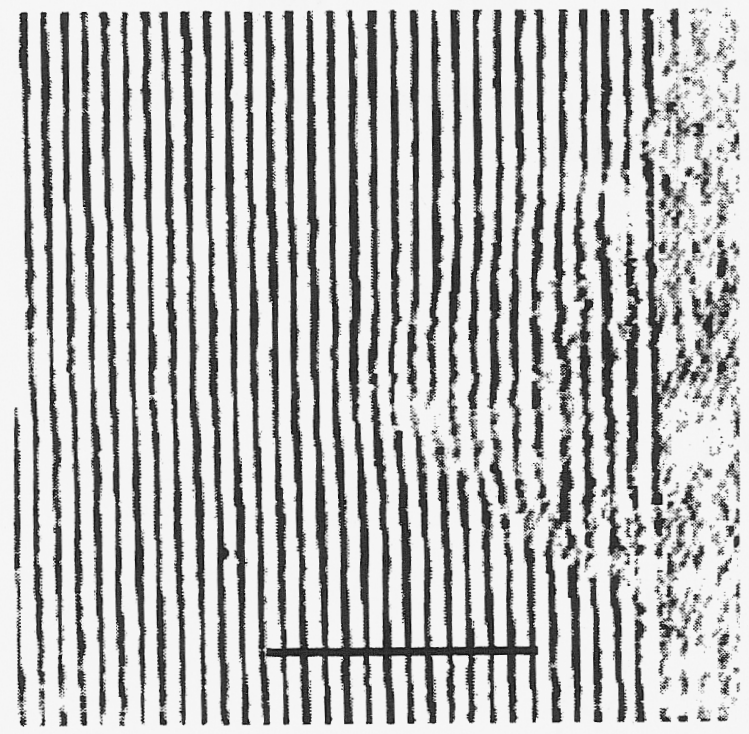

(A)
700 microns

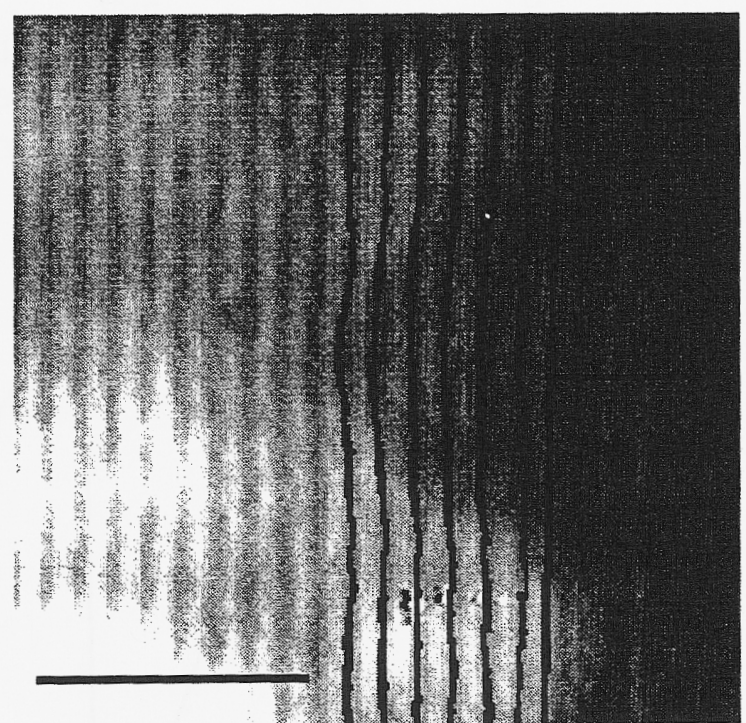

700 microns

(B)

Figure 3.4.5(A) and 3.4.5(B) show interferograms of $0.1 \mu \mathrm{m}$ Al film ablated by $3.33 \mathrm{ps}$ pulses at a peak front surface fluence of $1.77 \mathrm{~J} / \mathrm{cm}^{2}$. Here (A) is a $527 \mathrm{~nm}$ interferogram and $(B)$ is a $1053 \mathrm{~nm}$ interferogram. Both images captured $255 \mathrm{~ns}$ after arrival of incident laser pulse.

Figure 3.4.5 (A) and (B) shows an Al plume generated at far lower fluence than that of figure 3.4.4. In this case, the plume shadow is shaped similarly to that of the incident 
beam profile. This suggests that ablation at this fluence has similar dynamics to LIBA of $0.25 \mu \mathrm{m}$ films at much higher fluences. Figure 3.4.6 (A) and (B) shows the centerline neutral and electron density distributions.

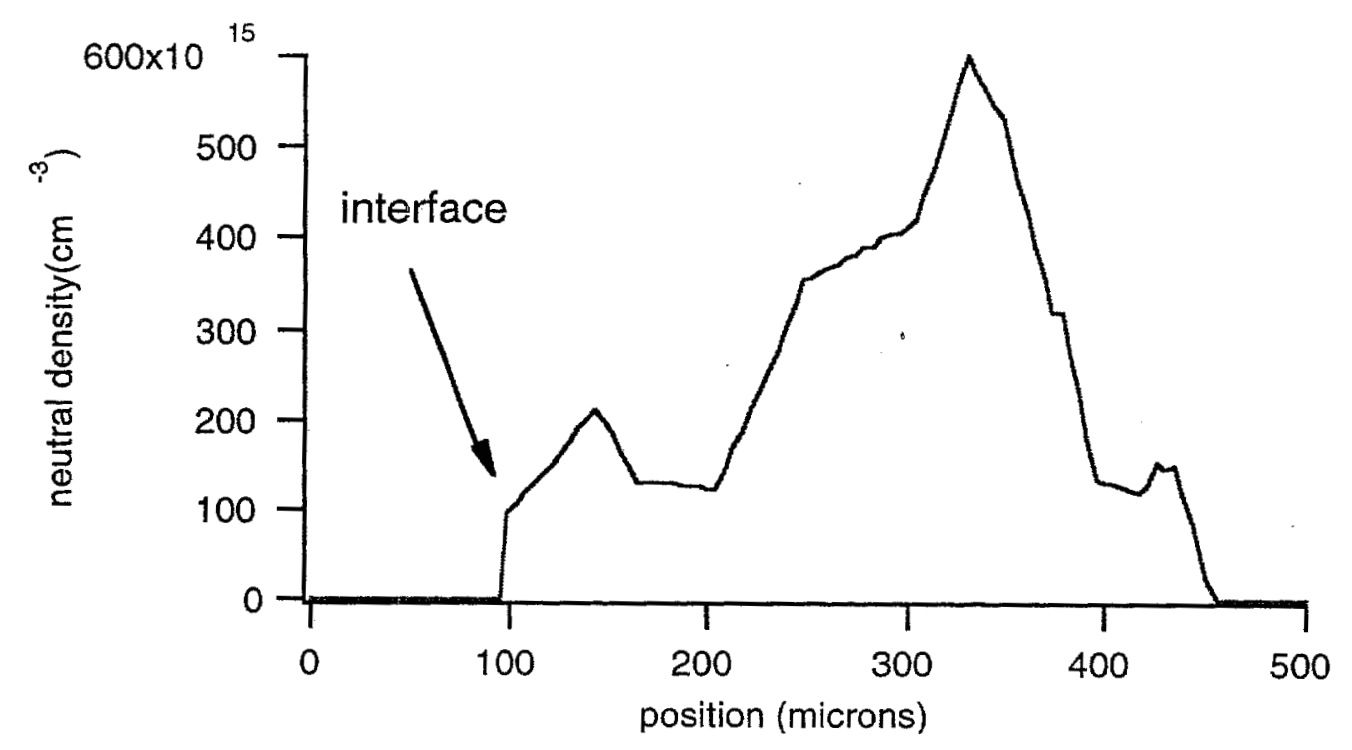

Figure 3.4.6(A). Plot of neutral density for a $0.1 \mu \mathrm{m} \mathrm{Al} \mathrm{film} \mathrm{ablated} \mathrm{by} 3.33$ ps ablation pulse $\left(1.77 \mathrm{~J} / \mathrm{cm}^{2}\right.$ peak front surface fluence).

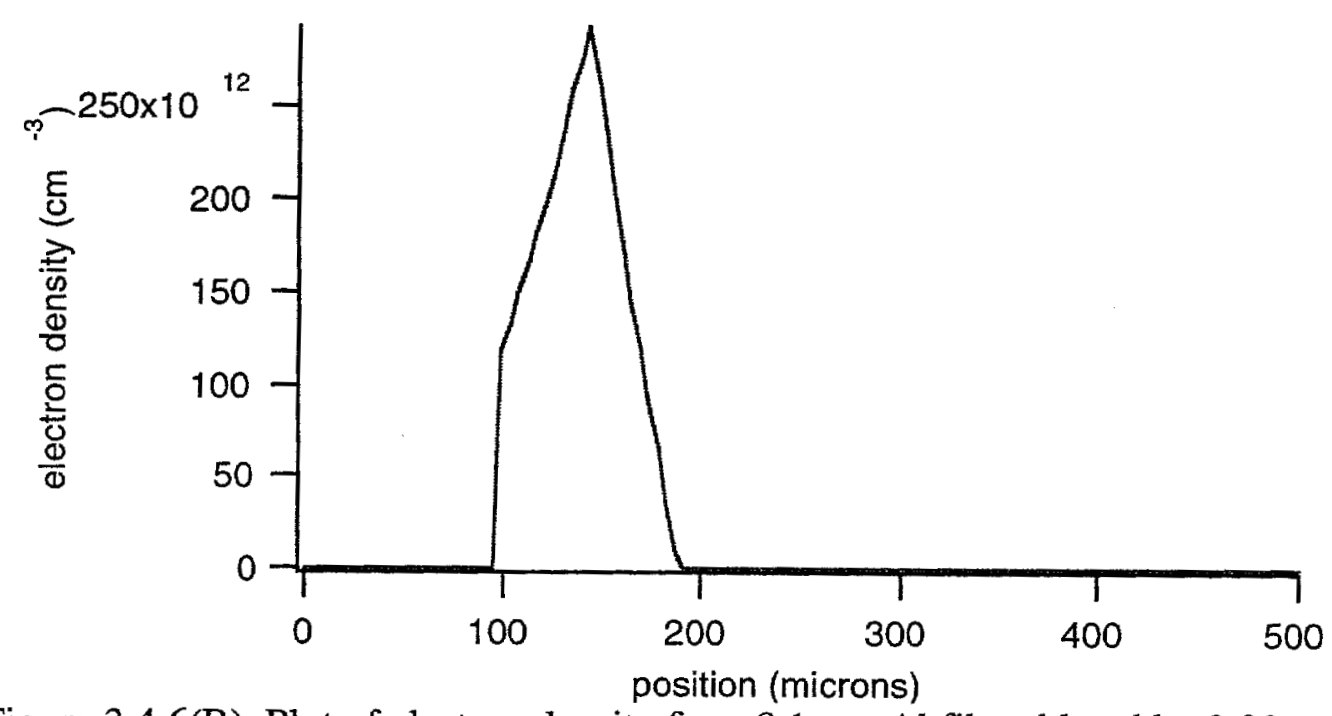

Figure 3.4.6(B). Plot of electron density for a $0.1 \mu \mathrm{m}$ Al film ablated by 3.33 ps ablation pulse $\left(1.77 \mathrm{~J} / \mathrm{cm}^{2}\right.$ peak front surface fluence). 
In this case, the relative distributions between electron and neutral atom density are not similar, and there is a noticeable separation between the peak of the electron distribution and the peak of the neutral atom distribution. Also, the level of ionization is lower (of order $5 \times 10^{-4}$ ). These observations suggest that the transverse asymmetries observed in Al plumes are dependent on the longitudinal temperature distribution of the Al film during ablation. One possible cause is complete melt-through and vaporization of the outer layers of the $\mathrm{Al}$ film just prior to ablation at the higher fluence level. If the temperature of the outer edge of $\mathrm{Al}$ film (i.e. the edge near the $\mathrm{Al} /$ vacuum interface) were to exceed the melting temperature and develop a significant vapor pressure, one may expect the ablation event to be similar to metal boiling away from the substrate surface in the case of very thin films. By contrast, at lower fluences $\left(1.77 \mathrm{~J} / \mathrm{cm}^{2}\right)$ or in cases where the film was too thick $(0.25 \mu \mathrm{m}$ or higher $)$ for heat to conduct entirely through the film thickness in the ablation time scale, one observes a symmetric plume with minimal expansion. Low fluence or thick Al films would also result in a hot interior film region near the interface where the laser energy has accumulated and a colder outer film region. In the higher fluence case shown by figure 3.4 .4 , however, we observe the asymmetric plume structure which suggests that the higher fluence caused the outer edge of the Al film to melt in the ablation time scale. A solution of the 1-D heat transport equation for a semi-infinite solid (see figure 3.4.7) with a time-dependent heat source at the boundary is shown below ${ }^{2}$.

$$
T(x, t) \approx \frac{F_{0} \kappa^{\frac{1}{2}} T}{K}\left\{\operatorname{ierfc}\left(\frac{x}{2 \kappa^{\frac{1}{2}}(t-T)^{\frac{1}{2}}}\right)\right\}
$$


Here, the time dependent heat source is a constant heat source, $F_{0}$, on for times $0<t^{\prime}<T, K$ is the thermal conductivity of the solid, and $\kappa$ is the thermal diffusivity $\left(0.975 \mathrm{~cm}^{2} / \mathrm{s}\right.$ for Al).

The expression above is valid for times $t>\mathrm{T}$. This solution can be used as a rough model of heat transport prior to ablation. Figure 3.4 .8 shows a plot of (2) for two values of $t$, with A equal to

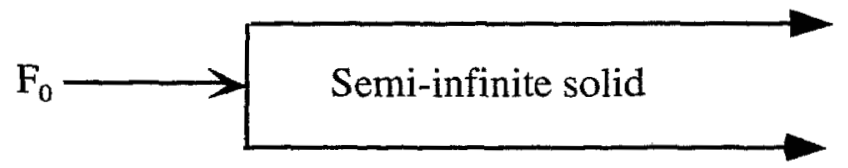

Figure 3.4.7. Sketch of 1-D semi-infinite heat transport problem with time dependent heat source, $F_{0}$.

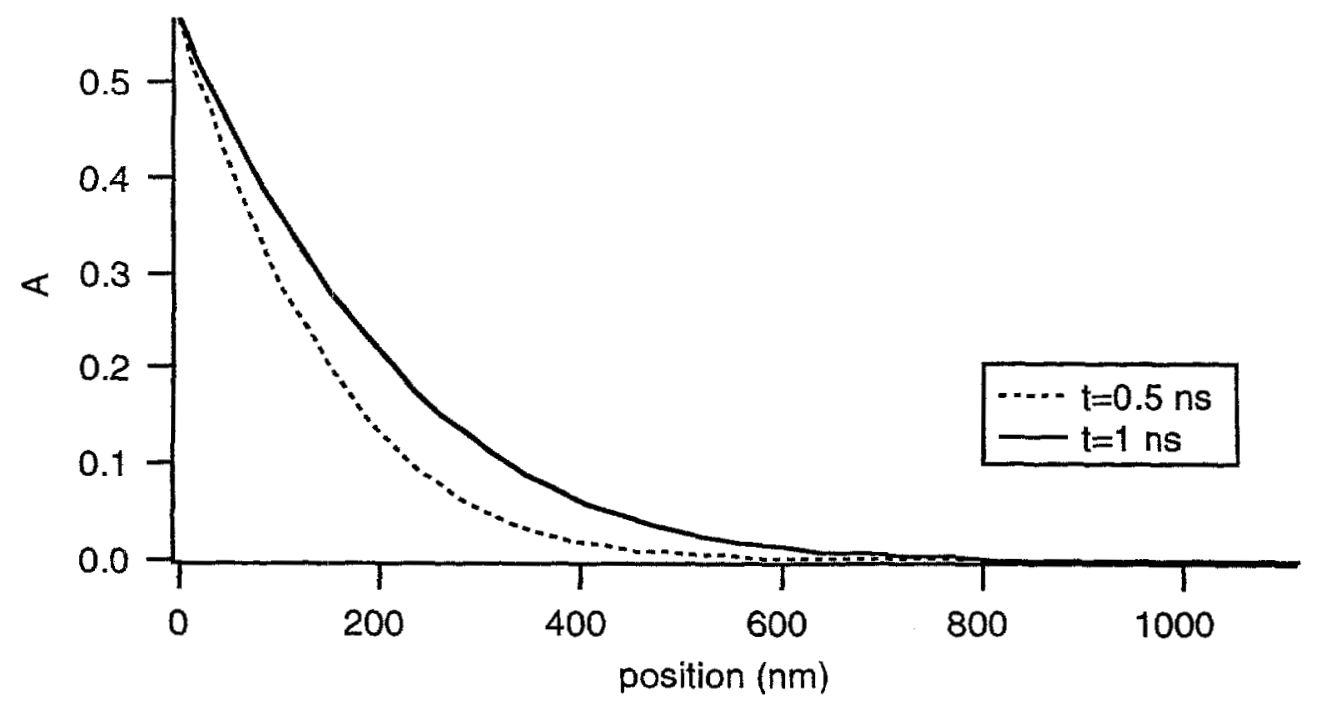

Figure 3.4.8. Plot of 1-D temperature distribution of semi-infinite Al slab after heating with time-dependent heat source. Here $T$ is given a value of $3.33 \mathrm{ps}$ and $\mathrm{x}$ variable in equation (1) is plotted along the position axis.

$\mathrm{T}(\mathrm{x}, \mathrm{t}) \mathrm{K} / \mathrm{F}_{0} \mathrm{~K}^{0.5} \mathrm{~T}$. In both curves shown in figure 3.4.8, the interface region is much higher in temperature than the outer regions. For low fluences near the ablation fluence threshold, the sharp difference in temperature between interface region and outer film regions would lead to the outer film region remaining solid while the interface region 
would melt and become ionized. At high fluences, the temperatures throughout the solid would exceed the melting temperature, and there would not be a sharp phase difference between from interface and outer regions.

\subsection{Spectral Observations}

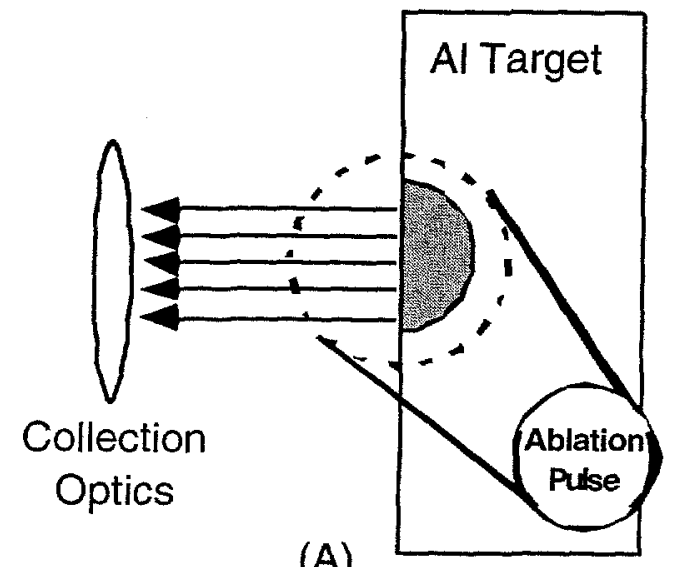

(A)

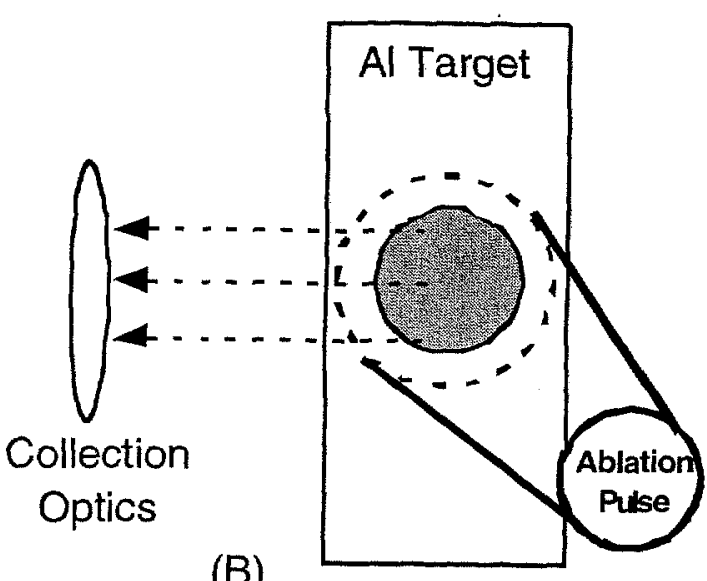

(B)

Figure 3.5.1. Diagram of "clipped" (A) and "unclipped" (B) target geometry

Using the spectrometer system described in previous sections, we gather the light emissions during the ablation of both uncoated BK-7 substrates and coated Al targets. Uncoated $\mathrm{Bk}-7$ substrates are used to study the spectral emissions caused by laserinduced substrate damage alone. Coated Al targets are used in two different target geometries to study Al plume emission. One geometry, the "clipped" geometry, involves placing the laser focal spot only partially on the $\mathrm{Al}$ target (see figure 3.5.1(A)). This assures that the high fluence central region of the incident beam heats material on the edge of the thin film. This geometry assures that the hottest $\mathrm{Al}$ region in the thin film is not separated from the collection optics by a colder $\mathrm{Al}$ film region. This then minimizes the absorption of the important spectral lines by colder aluminum atoms. The "unclipped" 
geometry, shown in figure 3.5.1(B), places the full laser spot on the $\mathrm{A} 1$ target. This geometry is identical to that used in the edge velocity study, and the emissions gathered from this experimental geometry allow the spectral monitoring of the "unclipped" LIBA experiments such as the edge velocity study and the plume redeposition studies.

\section{Uncoated BK-7 Substrate Spectra}

Previous sections detailed the importance of laser-induced substrate damage as an edge velocity limiting mechanism. Substrate damage such as that observed in these experiments readily cause spectral emission in the visual band. In figure 3.5 .2 , the visual spectra originating from the substrate of an uncoated BK-7 substrate is shown. Here, the spectrometer collection optics have been placed to optimize emissions from the substrate front surface. These uncoated slides were placed fully in the beam spot in the "unclipped" geometry shown in figure 3.5.1(B). The figure shows line emission superimposed onto a background continuum. A local maximum in the continuum appears in the region of $570 \mathrm{~nm}$ to $600 \mathrm{~nm}$, while a dip appears in the region around $750 \mathrm{~nm}$. Continuum emission is locally optimized again in the spectral region near $820 \mathrm{~nm}$. The general shape of this emission persists even at higher peak front surface fluences. Figure 3.5.3 shows the substrate damage spectra at much higher fluence, and while individual line heights may change, the distinctive shape remains consistent. This shape is very unlike a blackbody spectra, and one may expect this spectral shape to be the sum of many closely spaced spectral emission lines. Table 2.5.1 lists the large number of silicon spectral lines which may be present in the observed spectra. 


\begin{tabular}{|c|c|c|c|c|c|}
\hline wavelength $(\AA)$ & Ionization state & wavelength $(\AA)$ & Ionization state & wavelength $(\AA)$ & Ionization state \\
\hline 8502.22 & 1 & 7184.89 & 1 & 5753.63 & \\
\hline 8501.55 & 1 & 7165.55 & 1 & 5747.67 & 1 \\
\hline 8443.98 & 1 & 7164.69 & 1 & 5739.73 & 3 \\
\hline 8306.71 & 1 & 7034.9 & 1 & 5716.26 & 3 \\
\hline 8269.32 & 3 & 7017.65 & 1 & 5708.4 & 1 \\
\hline 8262.57 & 3 & 7017.28 & 1 & 5706.37 & 2 \\
\hline 8230.64 & 1 & 7005.88 & 1 & 5701.37 & 2 \\
\hline 8191.68 & 3 & 7003.57 & 1 & 5701.11 & 1 \\
\hline 8171.29 & 1 & 6976.52 & 1 & 5690.43 & 1 \\
\hline 8103.45 & 3 & 6848.57 & $\dagger$ & 5688.81 & 2 \\
\hline 8102.86 & 3 & 6829.82 & 2 & 5684.48 & 1 \\
\hline 8093.24 & 1 & 6818.45 & 2 & 5669.56 & 2 \\
\hline 8071.29 & 1 & 6741.64 & 1 & 5665.55 & 1 \\
\hline 8070.6 & 1 & 6721.85 & 1 & 5660.66 & 2 \\
\hline 8035.62 & 1 & 6699.38 & 2 & 5645.61 & 1 \\
\hline 8026.95 & 1 & 6671.88 & 2 & 5639.48 & 2 \\
\hline 7970.31 & 1 & 6555.46 & 1 & 5632.97 & 2 \\
\hline 7944 & 1 & 6527.2 & 1 & 5576.66 & 2 \\
\hline 7932.35 & 1 & 6526.61 & 1 & 5540.74 & 2 \\
\hline 7918.39 & 1 & 6371.36 & 2 & 5517.53 & 1 \\
\hline 7913.43 & 1 & 6347.1 & 2 & 5496.45 & 2 \\
\hline 7849.97 & 1 & 6331.95 & 1 & 5493.23 & 1 \\
\hline 7849.72 & 2 & 6254.85 & 1 & 5469.21 & 2 \\
\hline 7848.8 & 2 & 6254.19 & 1 & 5466.87 & 2 \\
\hline 7800.01 & 1 & 6244.47 & 1 & 5456.45 & 2 \\
\hline 7742.71 & 1 & 6243.81 & 1 & 5438.62 & 2 \\
\hline 7680.27 & 1 & 6239.63 & 2 & 5405.34 & 2 \\
\hline 7612.36 & 3 & 6238.29 & 1 & 5202.41 & 2 \\
\hline 7482.19 & 1 & 6237.32 & 1 & 5192.86 & 2 \\
\hline 7466.32 & 3 & 6155.13 & 1 & 5185.54 & 2 \\
\hline 7462.62 & 3 & 6145.01 & 1 & 5185.25 & 2 \\
\hline 7455.36 & 1 & 6142.49 & 1 & 5181.9 & 2 \\
\hline 7424.6 & 1 & 6131.85 & 1 & 5114.12 & 3 \\
\hline 7423.5 & 1 & 6131.57 & 1 & 5091.42 & 3 \\
\hline 7415.95 & 1 & 6125.02 & 1 & 5056.31 & 2 \\
\hline 7415.35 & 1 & 5978.93 & 2 & 5041.03 & 2 \\
\hline 7409.08 & 1 & 5957.56 & 2 & 5006.06 & 1 \\
\hline 7405.77 & 1 & 5948.55 & 1 & 4947.61 & 1 \\
\hline 7373 & 1 & 5915.22 & 2 & 4828.97 & 3 \\
\hline 7290.26 & 1 & 5898.79 & 3 & 4819.72 & 3 \\
\hline 7289.17 & 1 & 5873.76 & 1 & 4813.33 & 3 \\
\hline 7282.81 & 1 & 5868.4 & 2 & 4800.43 & 3 \\
\hline 7275.29 & 1 & 5846.13 & 2 & 4792.32 & 1 \\
\hline 7250.63 & 1 & 5806.74 & 2 & 4792.21 & 1 \\
\hline $7250: 14$ & 1 & 5800.47 & 2 & 4782.99 & 1 \\
\hline 7235.82 & 1 & 5797.86 & 1 & 4716.65 & 3 \\
\hline 7235.33 & 1 & 5793.07 & 1 & 4665.87 & 2 \\
\hline 7226.21 & 1 & 5780.38 & 1 & 4621.72 & 2 \\
\hline 7208.21 & 1 & 5772.15 & 1 & 4574.76 & 3 \\
\hline 7193.9 & 1 & 5762.98 & 1 & 4567.82 & 3 \\
\hline
\end{tabular}




$$
7193.58
$$

1

5754.22

1

4552.62

3

Table 2.5.1. A list of the Si I, Si II, and Si III spectral lines in the range lines of $455 \mathrm{~nm}$ to $851 \mathrm{~nm}^{3}$.

This table only includes Si I, Si II, and Si III lines, and over 150 possible lines are listed.

Since the spectrometer has a resolution of approximately $1 \mathrm{~nm}$, an understanding of the observed continuum spectra as a summation of many Si lines is reasonable. Two observed spectral lines, $590 \mathrm{~nm}$ and $820 \mathrm{~nm}$, show prominently in the spectra. The 590 $\mathrm{nm}$ line is potentially the sum of several strong silicon spectral lines. The lines $591.5 \mathrm{~nm}$ Si II (3s3p4s-3s3p4p), $589.9 \mathrm{~nm}$ Si III, 590nm Si III(3s5f-3s5g), 587.4nm Si I $\left(3 \mathrm{~s}^{3} \mathrm{p} 3-\right.$ $\left.3 s^{2} 3 p 5 p\right)$, and $586.8 n m$ Si II $(3 s 3 p 4 s-3 s 3 p 4 p)$ can all contribute to the observed line intensity at $590 \mathrm{~nm}^{4}$.

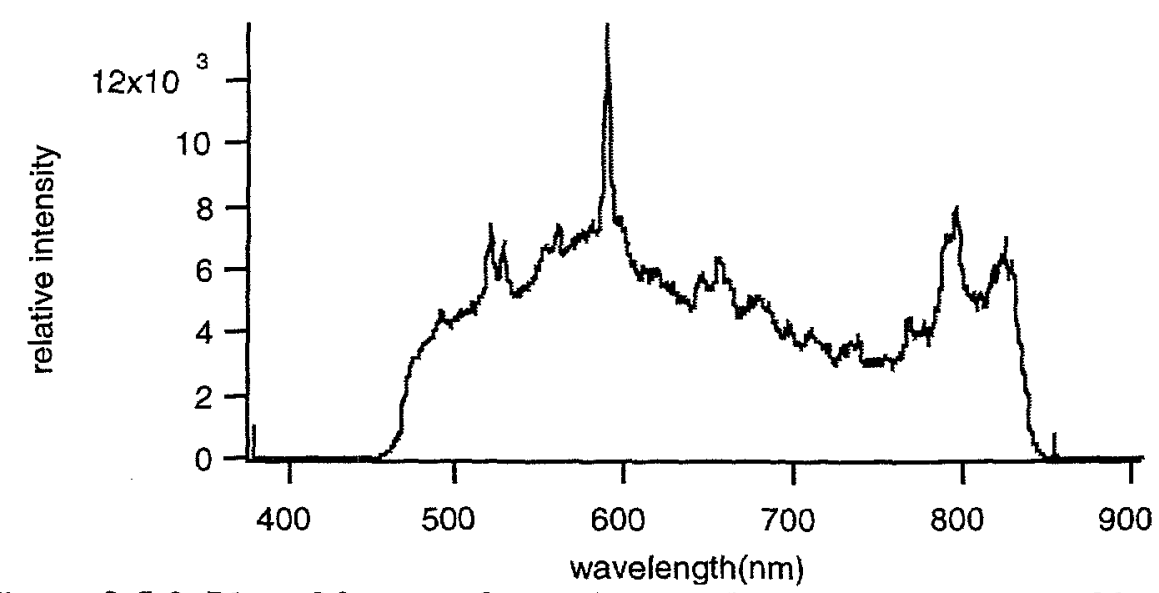

Figure 3.5.2. Plot of front surface substrate damage spectra caused by a $3.33 p$ s pulse with a peak fluence of $11.5 \mathrm{~J} / \mathrm{cm}^{2}$. 


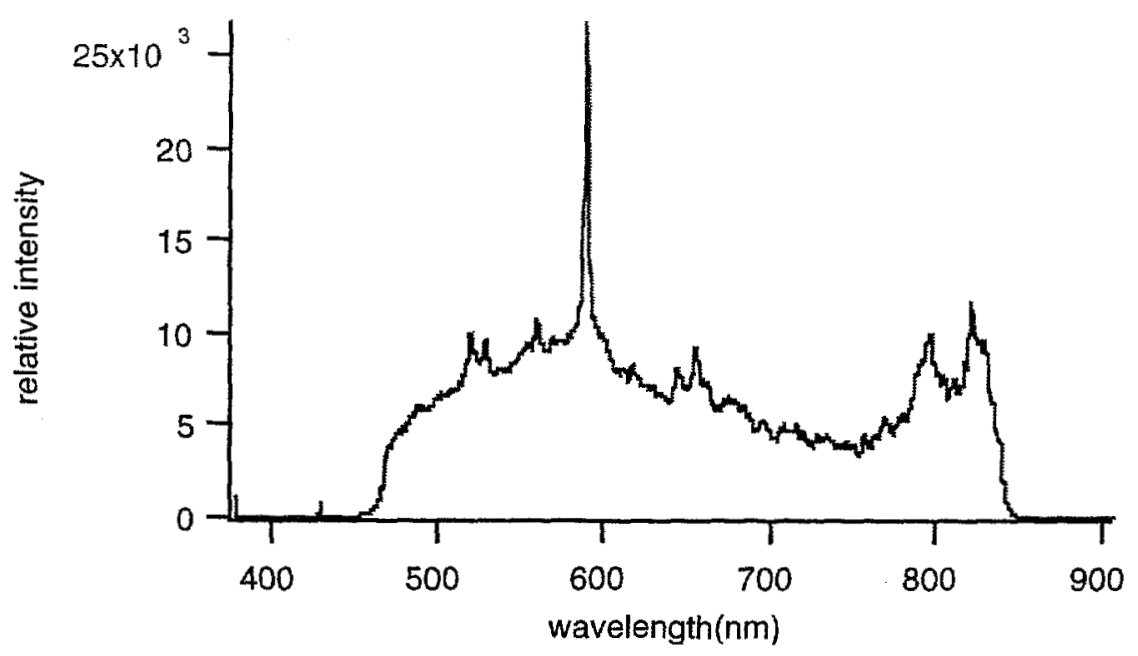

Figure 3.5.3. Plot of front surface substrate damage spectra caused by a 3.33ps pulse with a peak fluence of $31.1 \mathrm{~J} / \mathrm{cm}^{2}$.

The observed line intensity at $820 \mathrm{~nm}$ may also be the sum of spectral lines. The silicon spectral lines 819.2 Si III(3p5f-3p6g) 817.1 nm Si I, and 823.1 Si I $\left(3 \mathrm{~s} 3 \mathrm{p}^{3}-3 \mathrm{~s} 3 \mathrm{p}^{2} 5 \mathrm{p}\right)^{1}$ both contribute to the observed $820 \mathrm{~nm}$ line. Similarly, the line observed at $794 \mathrm{~nm}$ comes from the atomic lines $797.0 \mathrm{~nm} \mathrm{Si}$ I, $794.4 \mathrm{~nm}$ Si I, $791.3 \mathrm{~nm}$ Si I, $793.2 \mathrm{~nm} \mathrm{Si} \mathrm{I,} \mathrm{and} 791.8 \mathrm{~nm}$ SiI (all lines $3 \mathrm{p} 4 \mathrm{~d}-3 \mathrm{p} 5 \mathrm{~d})^{5}$. Since all targets, both uncoated substrate targets and Al coated targets, have a BK-7 substrate, this substrate damage spectra can be expected to contribute to the total spectral emission of the Al target during LIBA with laser fluences above the substrate damage threshold.

An additional feature of the spectra shown in figures 3.5.2 and 3.5.3 is the hard spectral cutoff at $460 \mathrm{~nm}$ and at $850 \mathrm{~nm}$. These features are attributed to the finite size of the redicon linear array in the spectrometer system. The shape of the continuum near these hard edges is determined by the relative wavelength-dependent response of both the diffraction grating and the redicon. Away from these edges, the continuum shape is 
attributed to the large number of spectral lines which are closely spaced and difficult to resolve in these spectrograms.

\section{Unclipped Al Target Spectra}

With the collection optics set to maximize gathering efficiency at the back surface of the Al target, LIBA-produced emissions were collected during the edge velocity experiments. The laser spot was placed on the target in the "unclipped" geometry, which causes the hot center region of the ablated plume to be surrounded by colder Al material. Figure 3.5.4 shows the spectrum from the $\mathrm{Al}$ plume generated by a $0.25 \mu \mathrm{m} \mathrm{Al} \mathrm{film}$ ablated by a 1.85 ps pulse.

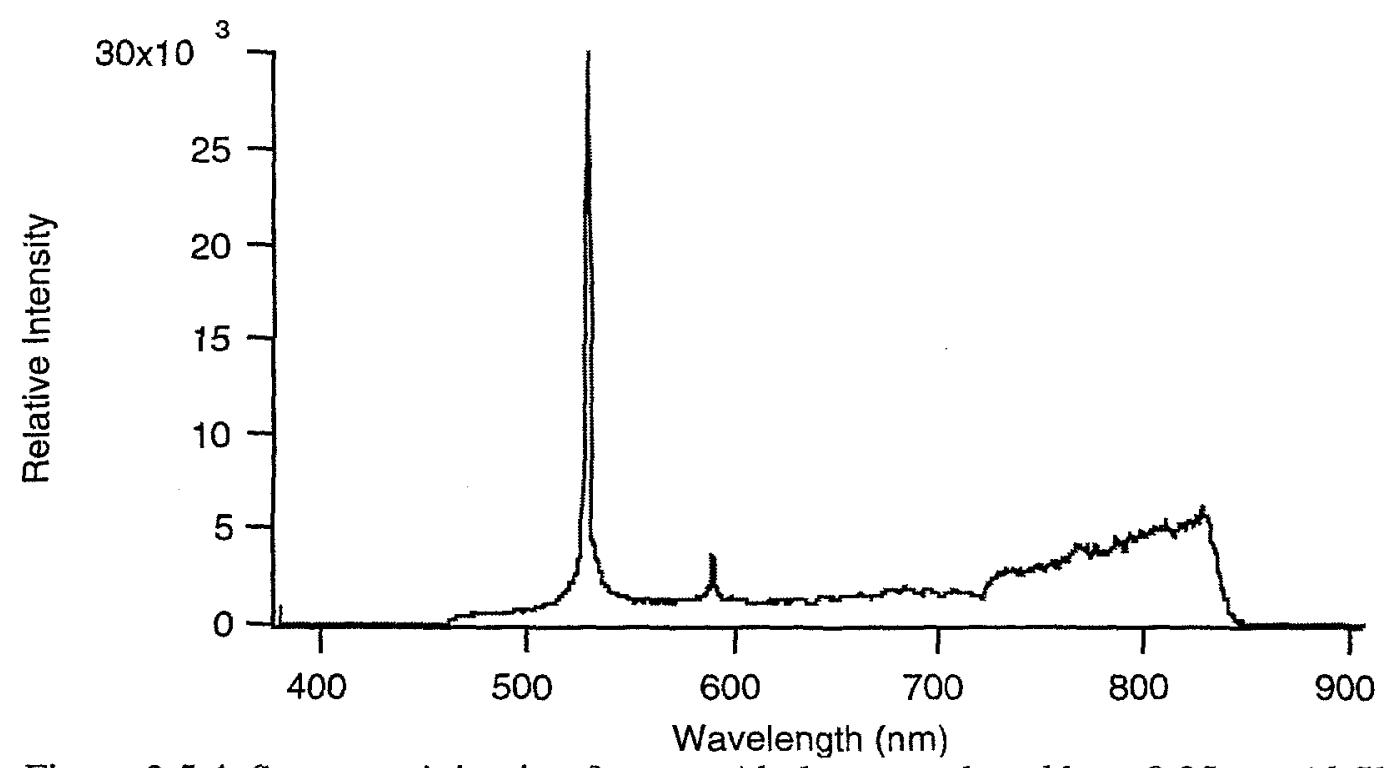

Figure 3.5.4. Spectra originating from an Al plume produced by a $0.25 \mu \mathrm{m}$ Al film ablated by a $1.85 \mathrm{ps}$ pulse $\left(19.0 \mathrm{~J} / \mathrm{cm}^{2}\right.$ peak fluence). The prominent signal at $527 \mathrm{~nm}$ is scattered light from the two-color probe pulse and is not part of the emission spectra.

Aside from the line at $590 \mathrm{~nm}$, the spectrum resembles a blackbody emission continuum for a temperature much less that $1 \mathrm{eV}$ (see figure 3.5.5). From the classical expression for black body emission spectrum ${ }^{6}$, one can see that, for a black body spectrum which appears to maximize at wavelengths larger than $1 \mu \mathrm{m}$ (such as figure 3.5.5), the 
temperature must be much less than $1 \mathrm{eV}$. For example, the background continuum which

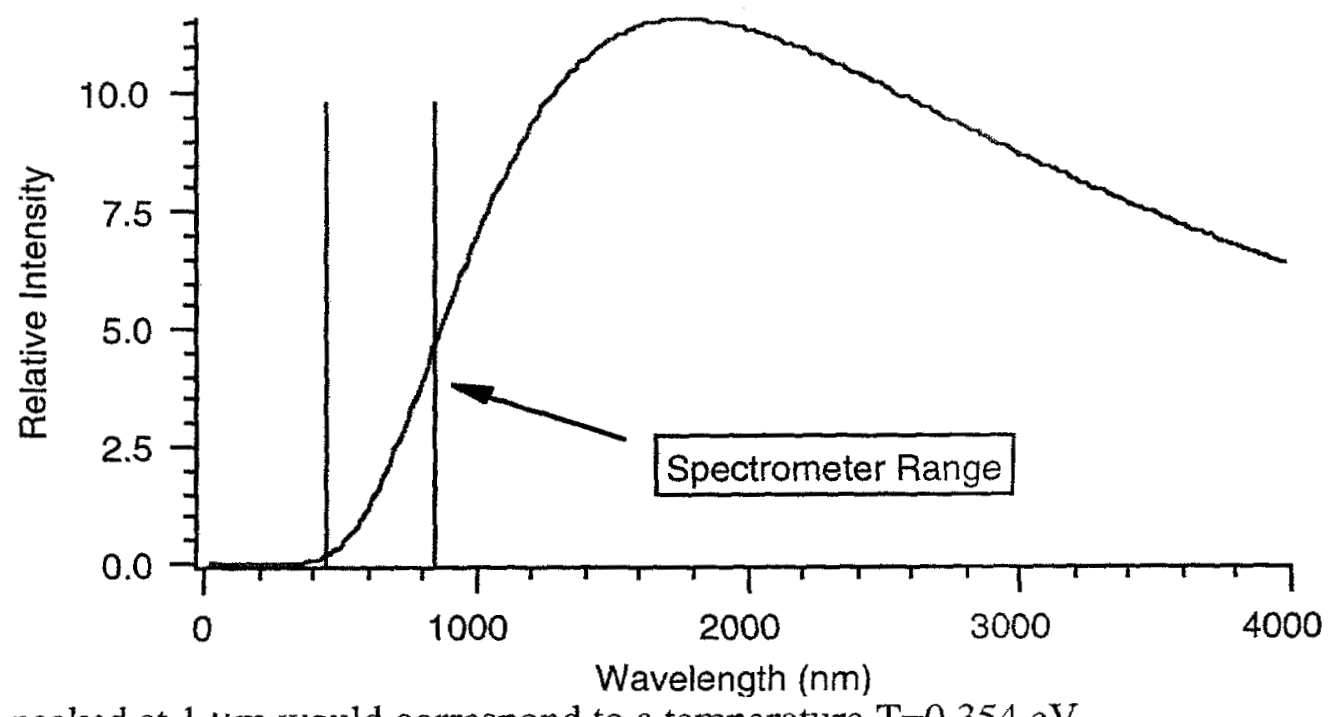

peaked at $1 \mu \mathrm{m}$ would correspond to a temperature $\mathrm{T}=0.354 \mathrm{eV}$.

Figure 3.5.5 Calculated continuum emission for a black body at $\mathrm{T}=0.25 \mathrm{eV}$.

Hence, a spectrum like that shown in figure 3.5.4 suggests that the time-averaged temperature of the plume is much less than $1 \mathrm{eV}$. The peak seen at $590 \mathrm{~nm}$, which is attributed to laser-induced substrate damage, is very similar to that shown in the substrate damage spectra, and this line therefore evidences the onset of substrate damage. We see a similar spectrum for the $\mathrm{Al}$ plume produced by a $0.5 \mu \mathrm{m}$ Al film ablated by a 3.33 ps pulse (see figure 3.5 .6 ). 


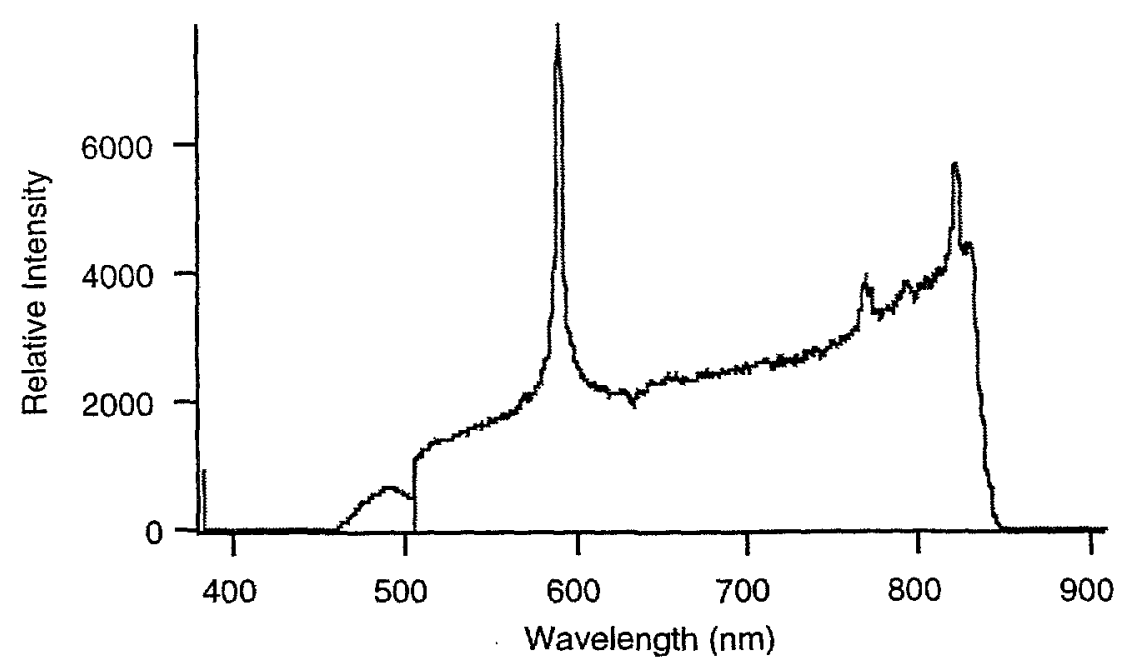

Figure 3.5.6. Spectra originating from an Al plume produced by a $0.50 \mu \mathrm{m}$ Al film ablated by a $3.33 \mathrm{ps}$ pulse $\left(12.3 \mathrm{~J} / \mathrm{cm}^{2}\right.$ peak fluence).

Again, we see a spectrum which resembles the short-wavelength section of a lowtemperature black body emission spectrum. In addition, we see spectral lines at $590 \mathrm{~nm}$ $774 \mathrm{~nm}$, and $820 \mathrm{~nm}$ corresponding to the substrate damage emission spectrum from the front surface. Because the collection optics are set to gather emissions from the back surface, light from the front surface is not as efficiently gathered. This is unlike the spectra gathered with uncoated $\mathrm{BK}-7$ substrates, in which case the collection optics were aligned to maximize collection of front surface emission. Therefore, at comparable incident fluences, substrate damage spectral line intensity from the front surface will be far lower in the case of a coated target spectra than in the substrate damage spectra shown in figures 3.5.2 and 3.5.3. At higher fluence, the substrate damage spectra becomes far more intense. Figure 3.5 .7 shows the spectrum from the ablation of a $0.25 \mu \mathrm{m}$ film by a 1.85 ps pulse at high fluence. Several spectral lines such as those at $590 \mathrm{~nm}, 655 \mathrm{~nm}$, and $820 \mathrm{~nm}$ are very 


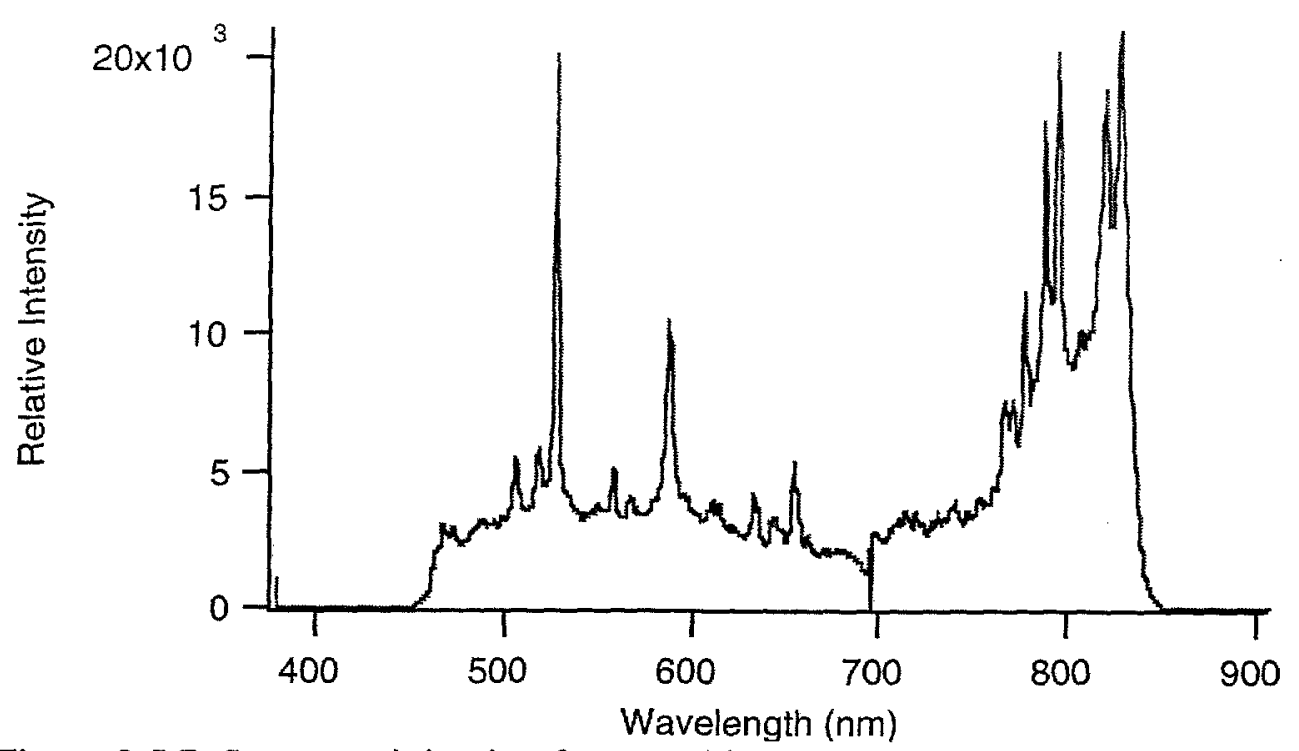

Figure 3.5.7. Spectra originating from an Al plume produced by a $0.25 \mu \mathrm{m} \mathrm{Al} \mathrm{film}$ ablated by a $1.85 \mathrm{ps}$ pulse $\left(151 \mathrm{~J} / \mathrm{cm}^{2}\right.$ peak fluence). The prominent signal at $527 \mathrm{~nm}$ is scattered light from the two-color probe pulse and is not part of the emission spectra.

intense relative to the background. The $655 \mathrm{~nm}$ line is identified as $655 \mathrm{~nm}$ Si I. Also, the general shape of the background no longer has a black body emission spectral shape. Instead, the background shape resembles that of a substrate damage curve. The Al plume black body curve should not change after front surface fluence has increased past the substrate damage fluence threshold. However, the front surface substrate damage will continue to increase with increasing fluence, and with increasing fluence, the observed spectrum will become dominated by the substrate damage spectrum originating from the front surface. Figure 3.5 .8 shows a similar spectrum for a $0.50 \mu \mathrm{m}$ film ablated by a 3.33 ps pulse at high fluence. This is consistent with a simple model proposed in the next chapter to explain the edge velocity behavior. According to this model, with increasing front surface fluence past the fluence threshold for laser-induced substrate damage, the total transmitted fluence arriving at the substrate/film interface is limited to a constant 
value. This would then cause the average plume temperature and velocity to also remain constant.

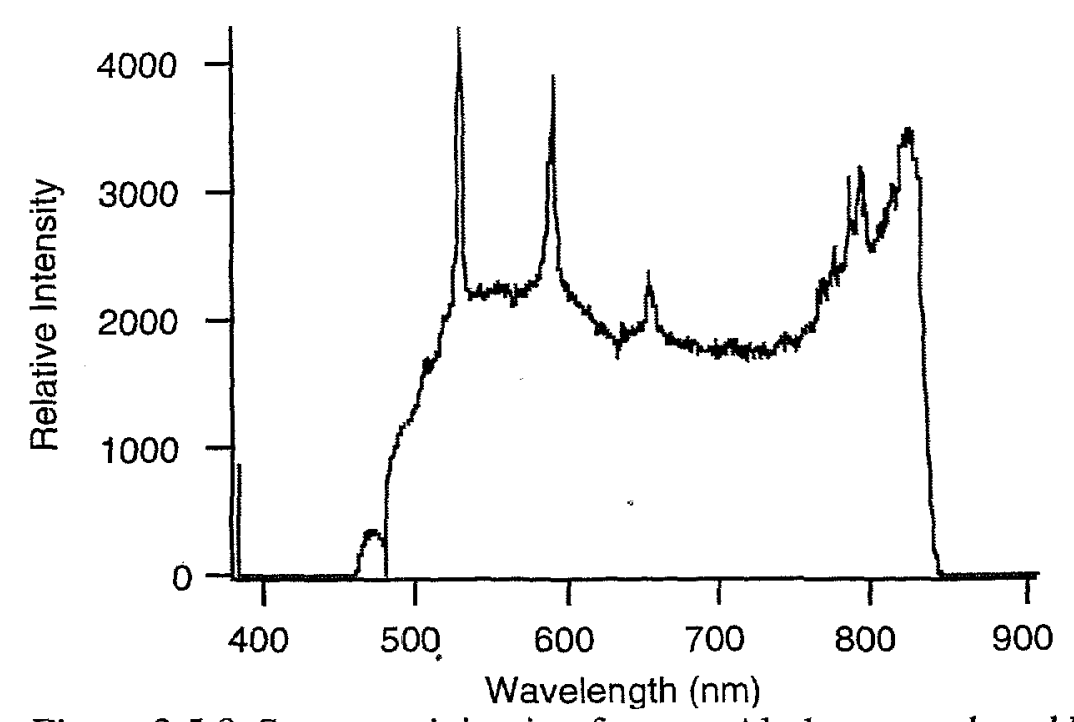

Figure 3.5.8. Spectra originating from an Al plume produced by a $0.50 \mu \mathrm{m}$ Al film ablated by a $3.33 \mathrm{ps}$ pulse $\left(151 \mathrm{~J} / \mathrm{cm}^{2}\right.$ peak fluence). The prominent signal at $527 \mathrm{~nm}$ is scattered light from the two-color probe pulse and is not part of the emission spectra.

Again, the intense substrate damage spectrum dominates the aluminum black body spectrum, and the observed spectrum has the distinctive substrate damage background spectral shape. The $590 \mathrm{~nm}$ line again shows prominently, as do the lines near $820 \mathrm{~nm}$. Since the $590 \mathrm{~nm}$ line is a strongest silicon line observed in this spectral study, it is a useful indicator of substrate damage during a LIBA experiment.

\section{Clipped Al Target Spectra}

In contrast with the unclipped Al target spectra, the clipped target spectra is not dominated by black body emission. Instead, the individual atomic lines for both $\mathrm{Al}$ and $\mathrm{Si}$ are well-separated and easily identifiable. Figure 3.5.9 shows a low fluence spectrum produced by ablating $0.25 \mu \mathrm{m}$ Al film with a 3.33 ps pulse. Note the large number of 


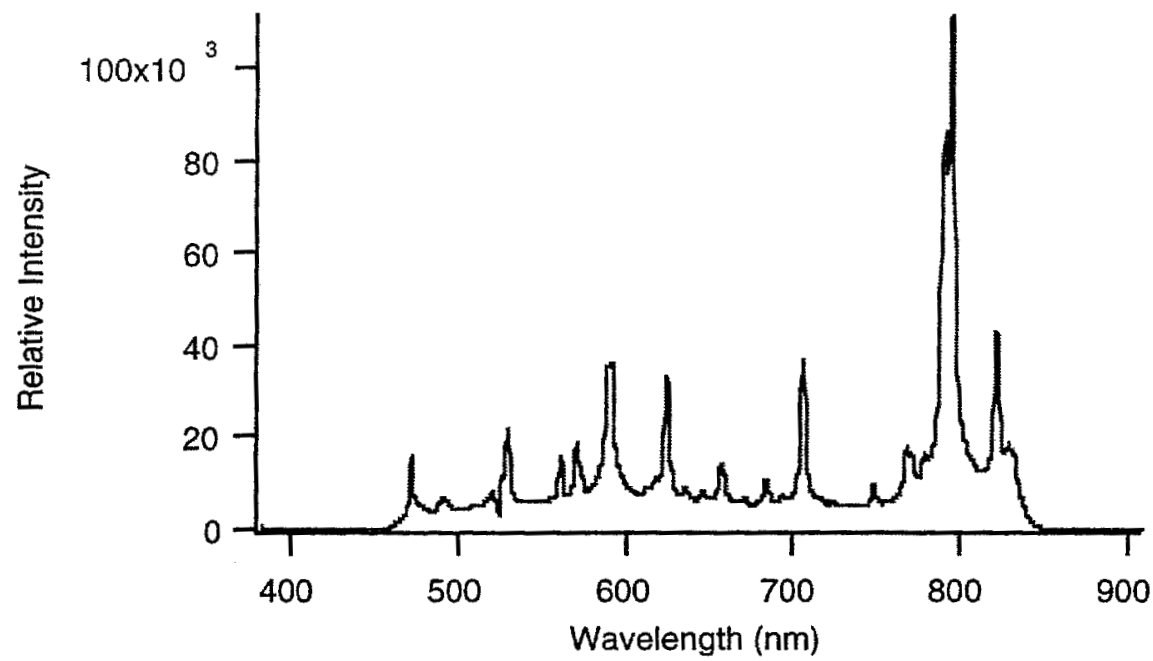

Figure 3.5.9. Spectra originating from an $\mathrm{Al}$ plume produced by a $0.25 \mu \mathrm{m} \mathrm{Al} \mathrm{film}$ ablated by a $3.33 \mathrm{ps}$ pulse $\left(12.3 \mathrm{~J} / \mathrm{cm}^{2}\right.$ peak fluence). The signal at $527 \mathrm{~nm}$ is scattered light from the two-color probe pulse and is not part of the emission spectra.

strong spectral lines in the spectrum. A chart of the observed spectral lines and the atomic species which can contribute to these lines is shown below (see table 3.5.2).

$\begin{array}{cccc}\begin{array}{c}\text { Observed Wavelength } \\ (\mathrm{nm})\end{array} & \text { Species } & \text { lonization State } & \text { Wavelength (angstroms) } \\ 472.9 & \text { al } & 3 & 4701.65 \\ 530.2 & \text { al } & 2 & 5316.07 \\ & \text { al } & 2 & 5312.32 \\ & \text { al } & 2 & 5285.85 \\ & \text { al } & 2 & 5283.77 \\ 561.5 & \text { al } & 2 & 5280.21 \\ 571.7 & \text { al } & 2 & 5593.23 \\ & \text { al } & 3 & 5722.65 \\ & \text { al } & 3 & 5696.47 \\ & \text { si } & 3 & 5739.73 \\ & \text { si } & 3 & 5716.26 \\ & \text { si } & 1 & 5708.4 \\ & \text { si } & 2 & 5706.37 \\ & \text { si } & 2 & 5701.37 \\ 591.1 & \text { si } & 1 & 5701.11 \\ & \text { si } & 1 & 5690.43 \\ & \text { si } & 2 & 5688.81 \\ & \text { si } & 2 & 5915.22 \\ & \text { si } & 3 & 5898.79\end{array}$




\begin{tabular}{|c|c|c|c|}
\hline Observed Wavelength & Species & Ionization State & $\begin{array}{l}\text { Wavelength (angstroms) } \\
\text { (cont.) }\end{array}$ \\
\hline 624.4 & al & 2 & 6243.35 \\
\hline 657.2 & si & 1 & 6555.46 \\
\hline \multirow[t]{3}{*}{683.8} & si & 1 & 6848.57 \\
\hline & si & 2 & 6829.82 \\
\hline & si & 2 & 6818.45 \\
\hline \multirow[t]{2}{*}{705.3} & al & 2 & 7042.06 \\
\hline & si & 1 & 7034.9 \\
\hline \multirow[t]{4}{*}{748.3} & si & 1 & 7482.19 \\
\hline & si & 3 & 7466.32 \\
\hline & si & 3 & 7462.62 \\
\hline & si & 1 & 7455.36 \\
\hline 767.7 & si & 1 & 7680.27 \\
\hline 770.3 & si & 1 & 7680.27 \\
\hline \multirow[t]{4}{*}{778.4} & si & 1 & 7800.01 \\
\hline & 0 & 1 & 7775.39 \\
\hline & 0 & 1 & 7774.17 \\
\hline & 0 & 1 & 7771.94 \\
\hline \multirow[t]{3}{*}{790.7} & si & 1 & 7932.35 \\
\hline & si & 1 & 7918.39 \\
\hline & si & 1 & 7913.43 \\
\hline \multirow[t]{5}{*}{794.3} & si & 1 & 7970.31 \\
\hline & si & 1 & 7944 \\
\hline & si & 1 & 7932.35 \\
\hline & si & 1 & 7918.39 \\
\hline & si & 1 & 7913.43 \\
\hline \multirow[t]{2}{*}{820.9} & si & 1 & 8230.64 \\
\hline & si & 3 & 8191.68 \\
\hline
\end{tabular}

Table 3.5.2. Table of observed spectral lines in spectrum presented in figure 3.5.8. Listed with each observed spectral line are the possible atomic spectral lines which contribute to the observed line intensity ${ }^{3}$.

Figure 3.5.10 illustrates the spectrum produced by another $0.25 \mu \mathrm{m} \mathrm{Al} \mathrm{film} \mathrm{when} \mathrm{ablated}$ with a $3.33 \mathrm{ps}$ pulse at high fluence. Note that Si lines such as $655.5 \mathrm{~nm}$ Si I and the various $\mathrm{Si}$ lines which contribute to the $820 \mathrm{~nm}$ and $794 \mathrm{~nm}$ lines gain in intensity relative to the $624 \mathrm{~nm} \mathrm{Al} \mathrm{II} \mathrm{with} \mathrm{increased} \mathrm{fluence.} \mathrm{This} \mathrm{effect} \mathrm{is} \mathrm{consistent} \mathrm{with} \mathrm{the} \mathrm{fluence-}$ 
limiting substrate damage model. According to this model, for increasing fluences above the substrate damage fluence threshold, the Al plume will have approximately the same temperature while the front substrate surface will continue to increase in temperature. In this case, the relative intensity of the various Al lines will not change, while the Si line emission will increase with incident laser fluence. A plot of the line intensity ratio of the observed 655.5nm Si I and the $624 \mathrm{Al}$ II line shows this behavior (see figure 3.5.11).

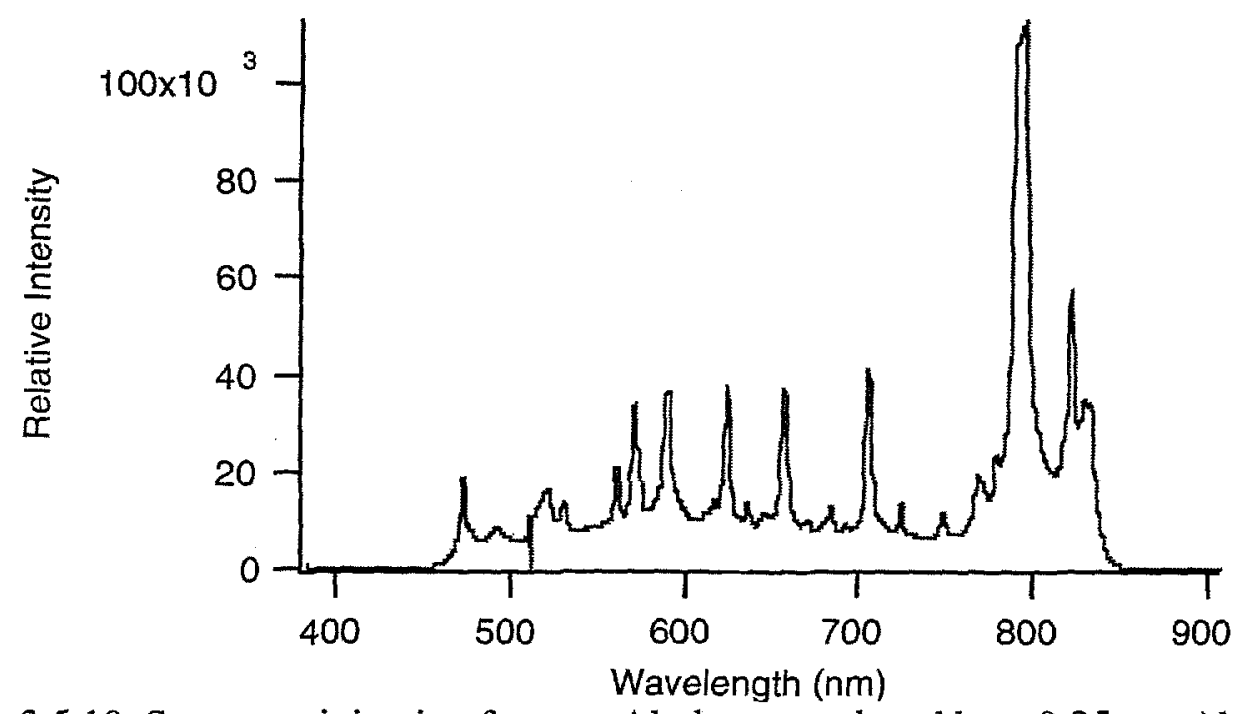

Figure 3.5.10. Spectra originating from an Al plume produced by a $0.25 \mu \mathrm{m} \mathrm{Al} \mathrm{film}$ ablated by a $3.33 \mathrm{ps}$ pulse $\left(25.7 \mathrm{~J} / \mathrm{cm}^{2}\right.$ peak fluence). The signal at $527 \mathrm{~nm}$ is scattered light from the two-color probe pulse and is not part of the emission spectra. 


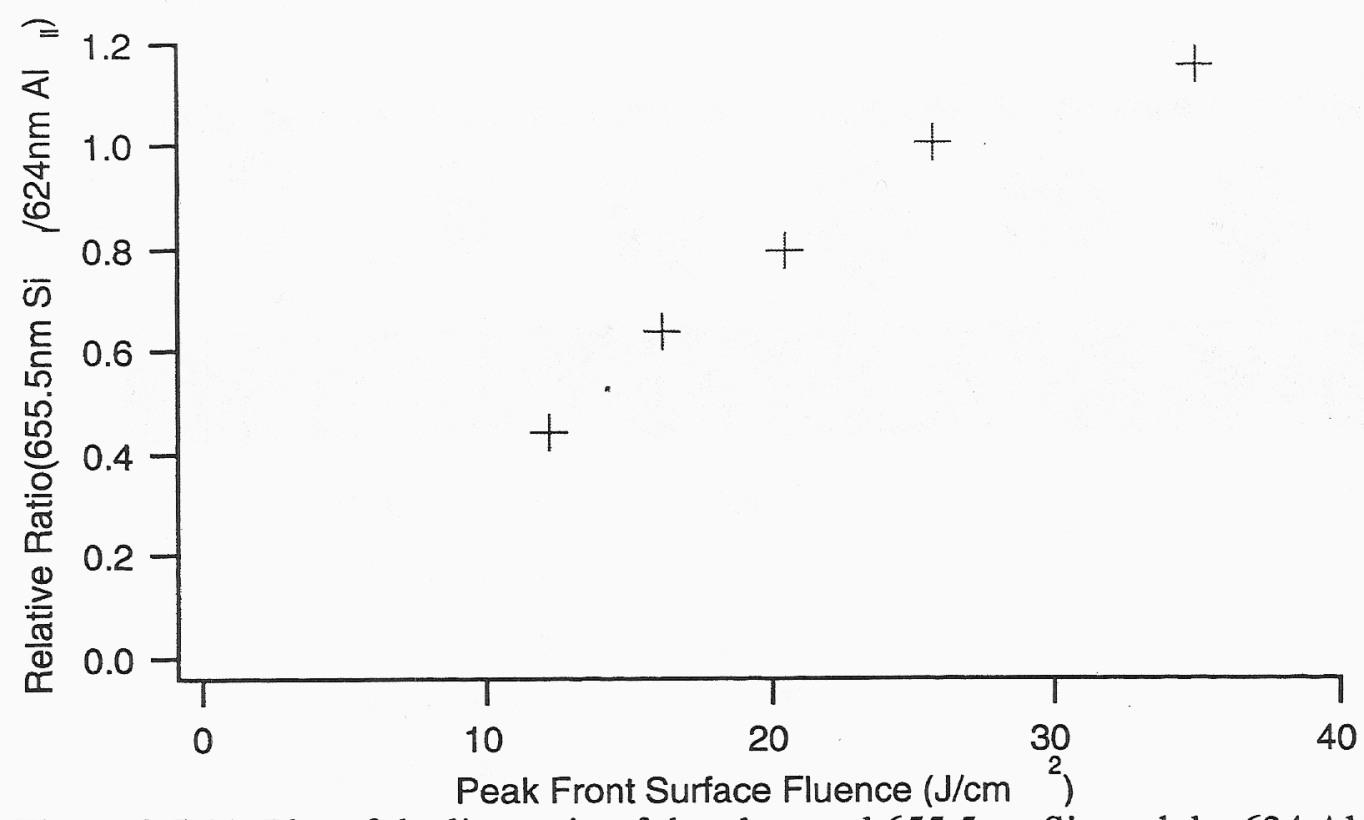

Figure 3.5.11. Plot of the line ratio of the observed $655.5 \mathrm{~nm} \mathrm{Si} \mathrm{I} \mathrm{and} \mathrm{the} 624 \mathrm{Al}$ II line as a function of front surface fluence for the $\mathrm{Al}$ plume produced by a $0.25 \mu \mathrm{m} \mathrm{Al}$ film ablated by a 3.33 ps pulse.

\subsection{Post-experimental Observations}

During LIBA, both the $\mathrm{Al}$ target and the receiving substrate are dramatically altered. The laser pulse removes the film on the $\mathrm{Al}$ target, and the ejected $\mathrm{Al}$ plume produces an aluminum deposit upon impact on the receiving substrate. Figure 3.6.1 shows an Al target after ablation. The black areas are holes in the thin film left by the ablation laser 


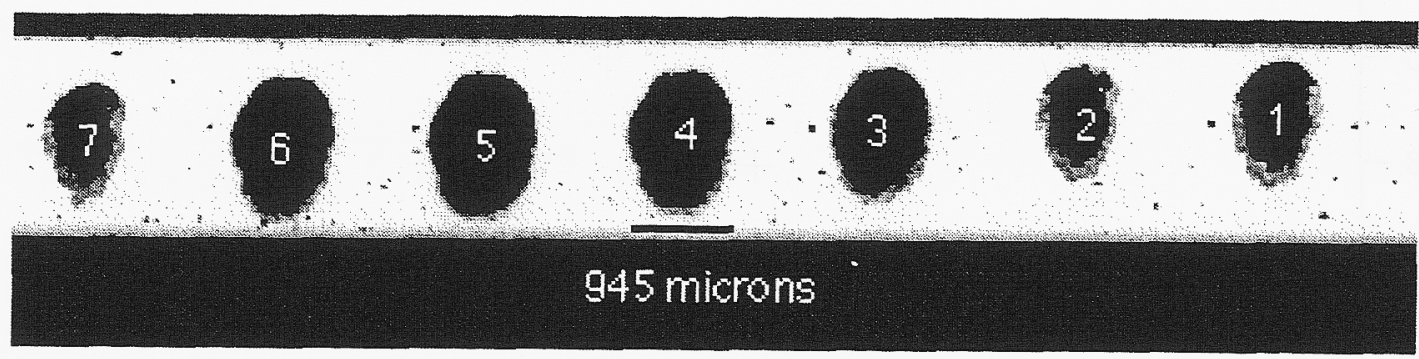

$\begin{array}{ll}\text { Shot \# } & \text { Peak Fluence } \\ 1 & 7.07 \mathrm{~J} / \mathrm{cm}^{2} \\ 2 & 5.07 \mathrm{~J} / \mathrm{cm}^{2} \\ 3 & 14.2 \mathrm{~J} / \mathrm{cm}^{2} \\ 4 & 21.5 \mathrm{~J} / \mathrm{cm}^{2} \\ 5 & 33.8 \mathrm{~J} / \mathrm{cm}^{2} \\ 6 & 17.9 \mathrm{~J} / \mathrm{cm}^{2} \\ 7 & 5.21 \mathrm{~J} / \mathrm{cm}^{2}\end{array}$

Figure 3.6.1. Picture of $0.25 \mu \mathrm{m} \mathrm{Al}$ target after LIBA. The Al film is lightly-colored, while the holes are black. Hole \# 4 has a transverse width of $945 \mu \mathrm{m}$, as shown above.

pulse. Note the ablation holes grow in size with increasing peak front surface fluence.

Figure 3.6.2 shows the transverse hole size of each ablation hole grows as a function of front surface fluence but not in a linear fashion. The hole growth saturates and reaches an asymptotic value at fluences above $15 \mathrm{~J} / \mathrm{cm}^{2}$. Since the incident laser pulse has an approximate gaussian distribution ( $402 \mu \mathrm{m}$ FWHM), one can calculate the ablation fluence threshold by calculating the fluence on the edge of an ablation hole. We use the following expression equating the threshold fluence to the fluence value of a spatial gaussian pulse at a position $1=W / 2$ away from the peak of the pulse to calculate the fluence threshold ${ }^{7}$ :

$$
J_{a b}=J_{p e a k} \exp \left[-\ln 2(W / D)^{2}\right]
$$


where $\mathrm{W}$ is the measured width of the ablation hole and D is the FWHM of the gaussian laser pulse.

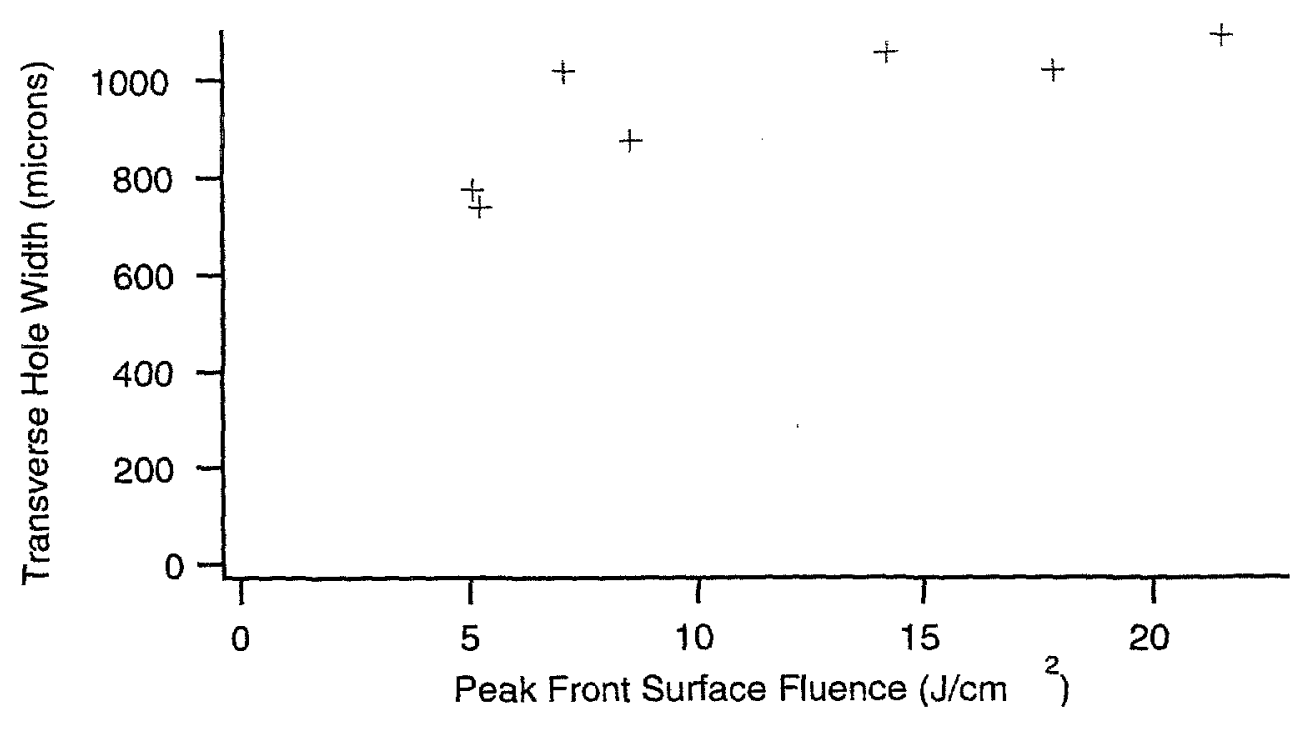

Figure 3.6.2. Plot of transverse hole size as a function of peak front surface fluence for ablations holes produced in $0.25 \mu \mathrm{m}$ films by 3.33 ps pulses.

This value gives an average threshold value of $0.65 \pm 0.211 \mathrm{~J} / \mathrm{cm}^{2}$ for $0.25 \mu \mathrm{m}$ films ablated by 3.33 ps pulses. Similarly, for $0.25 \mu \mathrm{m}$ films ablated by 1.85 ps pulses (see figure 3.6.3), the threshold is $0.859 \pm 0.465 \mathrm{~J} / \mathrm{cm}^{2}$. A measurement of the transverse hole width for $0.5 \mu \mathrm{m} \mathrm{Al}$ films ablated by 3.33 ps pulses produces a similar curve shown in Figure 3.6.4. The threshold for this case is $0.667 \pm 0.276 \mathrm{~J} / \mathrm{cm}^{2}$. This value is very close to that for the $0.25 \mu \mathrm{m}, 3.33$ ps case. In comparison to these threshold values, the threshold values yielded by the low fluence edge velocity study (see section 3.3.1) are listed below . 


$\begin{array}{llll}\text { Film Thickness } & \text { Pulsewidth } & \text { Low Fluence Threshold } & \text { Hole Width Threshold } \\ 0.25 \text { microns } & 3.33 p s & 1.2 \mathrm{~J} / \mathrm{cm}^{2} & 0.65+/-0.211 \mathrm{~J} / \mathrm{cm}^{2} \\ 0.25 \text { microns } & 1.85 \mathrm{ps} & 0.7 \mathrm{~J} / \mathrm{cm}^{2} & 0.859+/-0.465 \mathrm{~J} / \mathrm{cm}^{2} \\ 0.5 \text { microns } & 3.33 \mathrm{ps} & 0.68 \mathrm{~J} / \mathrm{cm}^{2} & 0.667+/-0.276 \mathrm{~J} / \mathrm{cm}^{2}\end{array}$

Table 3.6.1. Table of ablation fluence threshold estimates based on low-fluence edge velocity data and ablation hole size measurements.

The similarity between the fluence threshold in the $0.25 \mu \mathrm{m}, 3.33$ ps case and the $0.5 \mu \mathrm{m}$, 3.33 ps case suggests that film thickness does not affect the fluence threshold. In the "flyer plate" concept, the region near the interface acts as an explosive layer which removes the outer layers of the Al film, and with this conceptual physical event, one would expect that the fluence threshold would depend on the adhesive bond between the film and the substrate and the cohesive bonds between the $\mathrm{Al}$ atoms in the film. Of the two, the adhesive energy dominates the cohesive energy, and one would expect that the fluence threshold would be similar for both film thicknesses. The separation

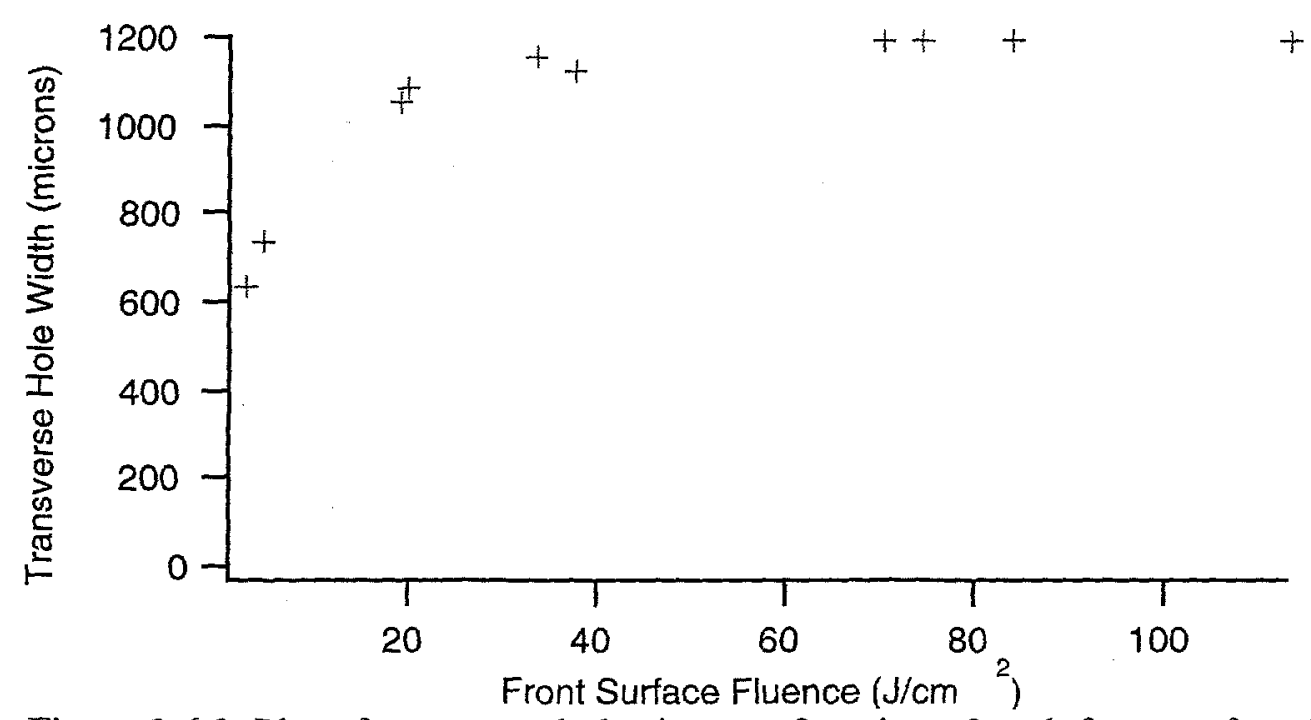

Figure 3.6.3. Plot of transverse hole size as a function of peak front surface fluence for ablations holes produced in $0.25 \mu \mathrm{m}$ films by 1.85 ps pulses. 
between the threshold estimates for the $0.25 \mu \mathrm{m}, 3.33$ ps case suggest that a square root functional form may not be a good model for edge velocity behavior at very low fluence. In this case, fluence estimates based on this model would not be reliable.

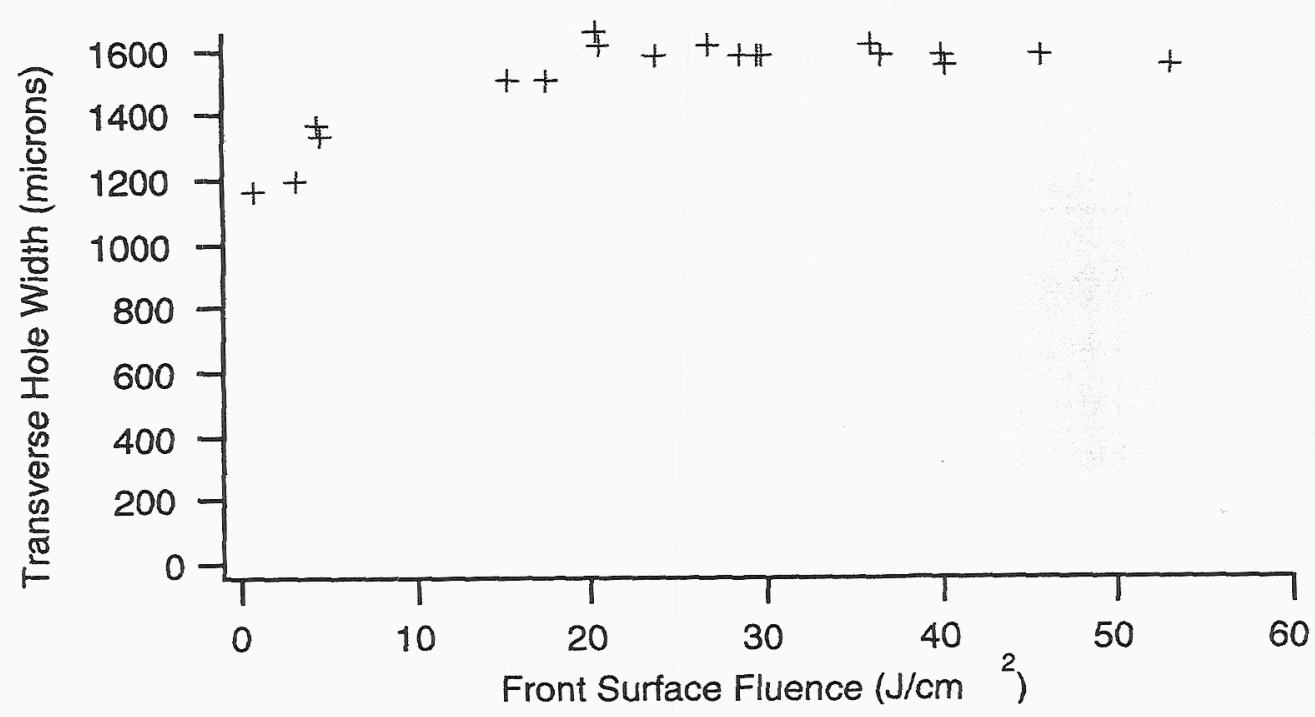

Figure 3.6.4. Plot of transverse hole size as a function of peak front surface fluence for ablations holes produced in $0.50 \mu \mathrm{m}$ films by 3.33 ps pulses.

On impact with the receiving substrate, the $\mathrm{Al}$ plume forms an $\mathrm{Al}$ deposit. The material distribution in this deposit can be measured by applying a profilometer to the deposit. Figure 3.6.5 shows the transverse height distribution of a series of Al deposits produced by ablating $0.50 \mu \mathrm{m}$ films by 3.33 ps pulses. 


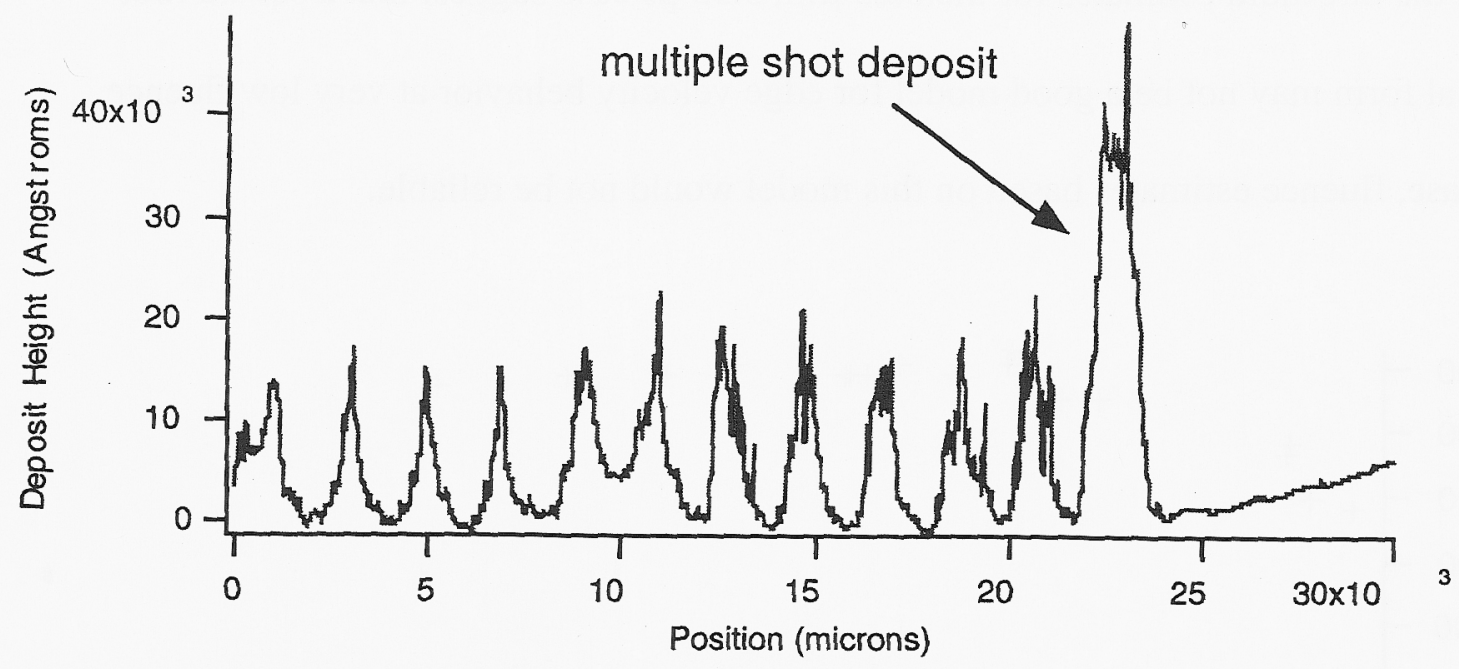

Figure 3.6.5. Plot of transverse height distribution for the case of $0.50 \mu \mathrm{m}$ films ablated by 3.33 ps pulses.

These deposits were formed by removal of the Al film from the target shown in figure 3.6.6.

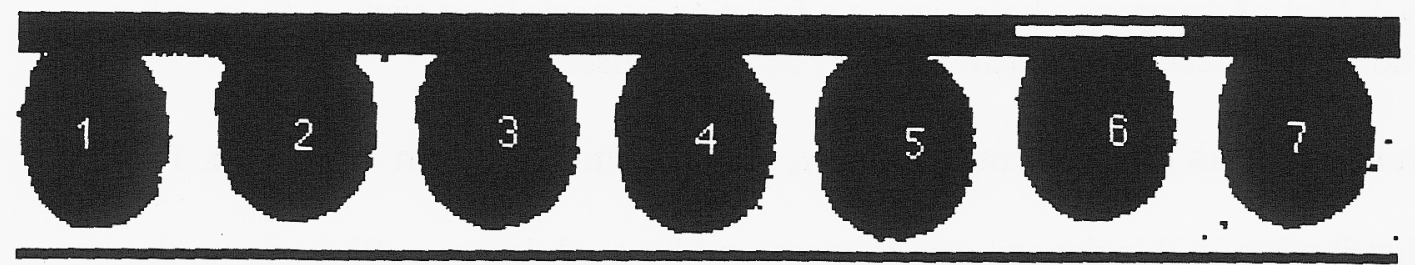

Figure 3.6.6. Photograph of the $0.50 \mu \mathrm{m} \mathrm{Al}$ target after ablation by $3.33 \mathrm{ps}$ laser pulses. The white bar above the target is a scale marker and is $1575 \mu \mathrm{m}$ in length.

A comparison of the deposit base width with the transverse hole width, given a substrateto-target distance of $4 \mathrm{~mm}$, gives an average divergence half-angle of $2.65 \pm 1.1$ degrees. This angle is the angle off normal measured from the edge of the ablation hole to the outer edge of the deposit. In the $0.25 \mu \mathrm{m}, 1.85$ ps case, the divergence angle is $1.19 \pm$ 2.24 degrees. In the $0.25 \mu \mathrm{m}, 3.33$ ps case, the divergence angle is $3.17 \pm 1.42$ degrees, and in the $0.5 \mu \mathrm{m}, 1.85 \mathrm{ps}$ case, the divergence angle is $2.01 \pm 1.5$ degrees. This low 
divergence is a useful asset in thin film deposition, since a low divergence angle allows precise deposition of metal. An estimate of the average material yield can be obtained by integrating over the height distribution in a deposit formed by multiple Al plumes. For example, one particular deposit was formed by $\mathrm{Al}$ plumes formed by $0.50 \mu \mathrm{m}$ films ablated with 3.33 ps case ( 5 separate plumes, generated by pulses with peak fluences of $43.3 \mathrm{~J} / \mathrm{cm}^{2}, 57 \mathrm{~J} / \mathrm{cm}^{2}, 49.2 \mathrm{~J} / \mathrm{cm}^{2}, 12.1 \mathrm{~J} / \mathrm{cm}^{2}$, and $12.3 \mathrm{~J} / \mathrm{cm}^{2}$ ). When the height is integrated over the total deposit area, it has $44 \%$ of the volume of the ejected Al film volume. Assuming that the deposit has the same density as the thin film, this shows the average constant density metal yield ratio is 0.44 . However, the deposit density is not necessarily equal to solid $\mathrm{Al}$ density, and one can readily expect the deposit density to be lower. For this reason, this yield value is presented as a coarse estimate of the actual value.

Simple measurements of the conductivity at both front and back surface of Al deposit give indications of the deposit material properties. Figure 3.6 .7 shows an interferogram formed by reflecting a $624 \mathrm{~nm}$ HeNe laser from both front and back of a receiving substrate. 


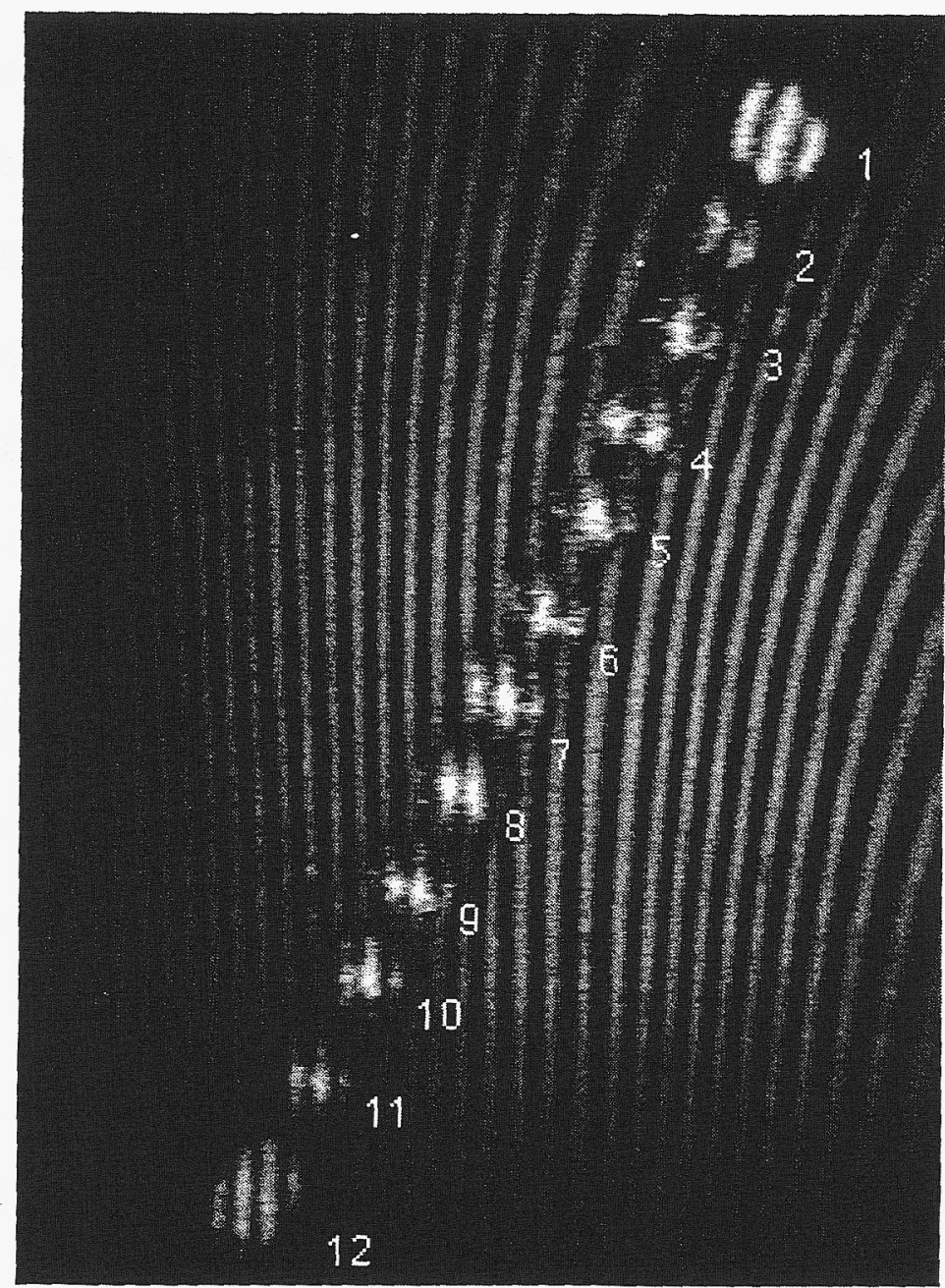

Figure 3.6.7. Interferogram of receiving substrate. The deposits were formed by ablating $0.5 \mu \mathrm{m} \mathrm{Al}$ films with 3.33 ps laser pulses. Spot \#1 is a deposit formed with multiple Al plumes. Here, the Al deposits are on the rear of the substrate, and this interferogram was taken looking at the front substrate surface.

The metal deposit is on the rear surface of the substrate. In areas away from the Al deposits, the fringe pattern formed by the reflected field from the front and back surfaces can be seen. The fringe pattern in the area of the deposit is a combination of two reflected electric fields. One field originates off the front substrate surface and the other reflection originates from the $\mathrm{Al}$ deposit region near the interface. The fringe pattern is phase-shifted by $\pi$ relative to the fringe pattern away from the deposit. This is consistent with the higher index of refraction expected with the deposit. The resistance properties of 
the multiple deposit spot shown in figure 3.6.7 were studied to obtain approximate values for the optical resistivity in an $\mathrm{Al}$ deposit. The fringe pattern intensity ratio, $\mathrm{I}_{\max } \mathrm{I}_{\min }$, in the area of the spot is measured from figure 3.6 .7 to be 2.63. This ratio is related to the reflectivity of the $\mathrm{Al}$ deposit by the expression below.

$$
\begin{aligned}
& \frac{I_{\max }}{I_{\min }} \approx 1+C \times R \\
& R \approx \frac{(1-n)^{2}}{(1+n)^{2}} \approx \frac{[1-c \sqrt{2 \sigma / \omega}]^{2}+[c \sqrt{\sigma / 2 \omega}]^{2}}{[1+c \sqrt{2 \sigma / \omega}]^{2}+[c \sqrt{\sigma / 2 \omega}]^{2}}
\end{aligned}
$$

where $\mathrm{C}$ is a constant for a given substrate and incident angle, $\mathrm{N}$ is the complex index of refraction for the $\mathrm{Al}$ deposit, and $\sigma$ is the conductivity of the $\mathrm{Al}$ deposit at the interface.

For a large real conductivity expected in a metal, one can expand $R$ into a power series of $1 / \mathrm{c}\left(2 \sigma_{\mathrm{r}} / \omega\right)^{0.5}$. This gives the following expression:

$$
R \approx \frac{[1-c \sqrt{2 \sigma / \omega}]^{2}+[c \sqrt{\sigma / 2 \omega}]^{2}}{[1+c \sqrt{2 \sigma / \omega}]^{2}+[c \sqrt{\sigma / 2 \omega}]^{2}} \approx \frac{1-1 / c \sqrt{2 \sigma / \omega}}{1+1 / c \sqrt{2 \sigma / \omega}} \approx 1-\left(\frac{2 \omega \varepsilon_{0}}{\sigma_{r}}\right)^{\frac{1}{2}}
$$

Using (3), we calculate the real optical conductivity of the interface layer at $624 \mathrm{~nm}, \sigma_{\mathrm{r}}$, given the fringe pattern ratio shown above. This value is found to be approximately $8.91 \times 10^{6}$ (ohm-meter $)^{-1}$, a value a factor of three below the solid Al optical conductivity at room temperature $\left.\left(2.94 \times 10^{7} \text { (ohm-meter }\right)^{-1}\right)$. In addition, a DC measurement of the resistivity of the outer deposit layer away from the interface was done by applying simple resistance probes to a series of deposits formed by ablating $0.5 \mu \mathrm{m} \mathrm{Al} \mathrm{films} \mathrm{with} \mathrm{both}$ $1.85 \mathrm{ps}$ and 3.33 ps pulses. Although the inhomogeneous and rough nature of the deposit outer surface introduces a large amount of uncertainty in the measurement, the average DC resistivity value of the deposits is $274 \pm 159 \mu \Omega \bullet \mathrm{cm}$. This resistivity value is two orders of magnitude above the $\mathrm{DC}$ resistivity for solid $\mathrm{Al}$ at room temperature $(2.5$ $\mu \Omega \bullet \mathrm{cm})^{8}$. This resistivity value is also equivalent to a DC conductivity of $3.65 \times 10^{5}$ 
(ohm-meter) $)^{-1}$. Comparing the real AC conductivity of the interface region of the deposit to the DC conductivity of the outer deposit layer, one can get an estimate of the collisionality of the outer deposit layer. Assuming the electron density in both regions is equal, the ratio of real $\mathrm{AC}$ conductivity to $\mathrm{DC}$ conductivity is shown in the expression below.

$$
\frac{\sigma_{\text {real }}^{a c}}{\sigma_{d c}} \approx \frac{v}{\omega}
$$

This ratio leads to a value for collision frequency of $7.37 \times 10^{16} \mathrm{~s}^{-1}$.

Scotch tape tests were performed on the receiving substrates to test deposit adhesion strength. The test consists of laying a piece of adhesive Scotch tape over the $\mathrm{Al}$ deposit and then removing the tape by applying normal force away from the substrate on one edge of the film. If noticeable $\mathrm{Al}$ material is removed from the substrate, the deposit fails this test. Al deposits formed by LIBA in this pulse regime pass this test at target-tosubstrate distances up to $6.31 \mathrm{~mm}$. Beyond this distance, detailed measurements were not undertaken, although cursory observations of Al deposits accidentally formed on optics and debris shields show the deposits can pass the scotch tape test even when recollected several $\mathrm{cm}$ away from the Al target.

\footnotetext{
${ }^{1}$ B.C.Stuart, M.D. Feit, S. Herman, A.M. Rubenchik, B.W. Shore, and M.D. Perry, Phys. Rev. B, 531749 (1996).

${ }^{2}$ H.S. Carslaw and J.C. Jaeger, Conduction of Heat in Solids [Oxford, New York, 1959], p.76.

${ }^{3}$ A.N. Zaidel, Tables of Spectral Lines (Phenum, New York, 1970), p. 654.

${ }^{4}$ S. Bashkin and J. Stoner, Atomic Energy Levels and Grotian Diagrams, (North Holland, New York, 1975), p.490.

${ }^{5}$ W.L. Wiese, M.W. Smith, and B.M. Miles, Atomic Transition Probablilities Volume II Sodium Through Calcium, (National Bureau of Standards, Washington D.C., 1969), p. 71.

${ }^{6} \mathrm{~F}$. Reif, Fundamentals of Statistical and Thermal Physics, (McGraw-Hill, New York, 1965), p. 388.

${ }^{7}$ A. B. Bullock, P.R. Bolton, and F.J. Mayer, J. Appl. Phys. 82 (4), 1828 (1997).

${ }^{8}$ Y. S. Touloukian and C.Y. Ho, "Properties of Aluminum and Aluminum Alloys," Report 21, Thermophysical Properties Research Center, Purdue University, 1973.
} 


\section{CHAPTER 4: ANALYSIS AND DISCUSSION OF LIBA RESULTS}

\subsection{Introduction}

A more detailed discussion of the LIBA experimental results is important to improve understanding and appreciation of picosecond LIBA as both a physical process and an engineering technique. To better understand the experimental results, both calculations and computer simulations were used. The edge velocity saturation effect was studied by calculating laser fluence transmission through the substrate as a function of fluence in the substrate damage fluence regime. A simple laser/plasma absorption calculation using the Helmholtz equation was also done to help understand the laser absorption of the $\mathrm{Al}$ film during the laser/matter interaction timescale. Using the substrate damage model, HYADES, a hydrodynamic and heat transport code, was utilized to produce simulations of LIBA edge velocity. Plume density, pressure, and temperature were also simulated by HYADES as a function of time and incident fluence in early time. The observed emission spectra of $\mathrm{Al}$ plumes produced in the clipped geometry was studied to get estimates of the average temperature, and these estimates are compared to the HYADES simulated temperature. The practical aspects of picoseond LIBA are also discussed. 


\subsection{Laser Fluence Transmission during Laser-Induced Substrate Damage}

To understand the observed relationship between edge velocity and incident fluence, we must understand the laser interaction with the aluminum target (see figure 4.2.1). In addition to the laser energy absorption at the thin film/substrate interface, we must also understand the fluence-limiting process caused by laser-induced ionization at the substrate. Formation of critical electron densities at the substrate by the leading edge of the ablation pulse would scatter the trailing elements of the pulse, thereby limiting the total transmitted fluence through the substrate.

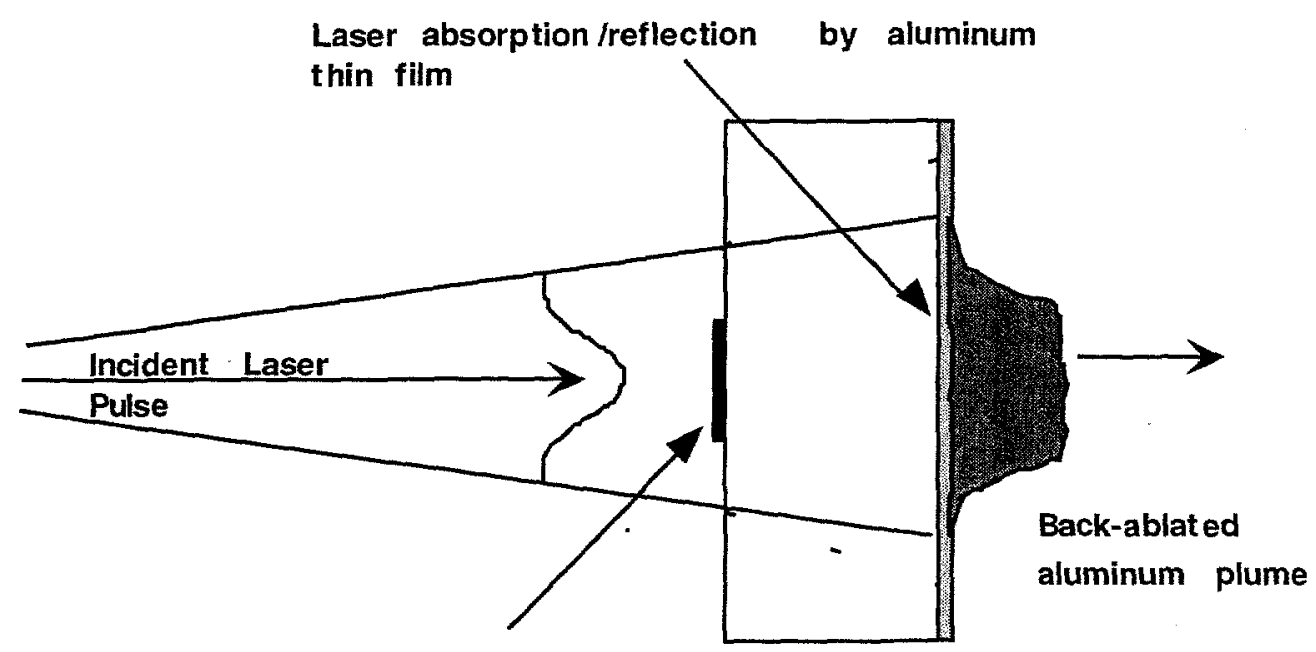
Laser-Induced
lonization at Front
Substrate Surface

Figure 4.2.1. Diagram of laser interaction with aluminum target

We compare our uncoated BK-7 transmission results with a simple man's model of transmission losses caused by laser-induced substrate in the substrate. At laser 
intensities of $1 \mathrm{TW} / \mathrm{cm}^{2}$, even when multiphoton ionization is negligible, avalanche ionization is initiated within a few femtoseconds after the arrival of the laser pulse at the substrate front surface ${ }^{1}$. For simplicity, we assume that a laser-generated plasma forms at the substrate front surface during the leading edge of the pulse. In this intensity regime, avalanche ionization, seeded by electrons produced by multiphoton ionization, dominates the substrate breakdown, so the electron ionization rate equation includes both mechanisms as shown below ${ }^{1}$.

$$
\frac{\partial n_{e}}{\partial t}=\beta(I) n_{e}+P(I)
$$

Here $\beta(\mathrm{I}) \mathrm{n}_{\mathrm{e}}$ is the avalanche ionization term and $\mathrm{P}(\mathrm{I})$ is the eight-photon multiphoton ionization term. Also, $\beta(I)=\mathrm{AI}$, where $\mathrm{A}$ is an adjustable parameter, and $\mathrm{P}(\mathrm{I})=9.52 \times 10^{10} \mathrm{I}(\mathrm{t})^{8} \mathrm{~cm}^{-3} \mathrm{psec}^{-1}$.

Our model assumes that transmission through the substrate surface is determined solely by the plasma generated at the substrate/vacuum interface. At this intensity regime, the primary laser absorption mechanism is inverse bremsstrahlung, and therefore laser absorption in the plasma maximizes near the critical surface ${ }^{1}$. Since electron density grows exponentially in time, the absorption experienced by the pulse is negligible when $\mathrm{n}(\mathrm{t})$ is lower than $10^{21} \mathrm{~cm}^{-3}$. When $\mathrm{n}(\mathrm{t})$ exceeds the critical density, a reflective plasma layer forms at the interface. Therefore it is assumed for simplicity that electron densities below critical density, $\mathrm{n}_{\mathrm{cr}}$, do not significantly alter the transmission and that electron densities at or above critical density $\left(10^{21} \mathrm{~cm}^{-3}\right)$ immediately truncate the transmitted laser pulse. In this case, the laser pulse transmitted through the front substrate surface is truncated at $\mathrm{t}_{0}$, the time at which electron density reaches critical level. Transmission is then written as; 


$$
\begin{aligned}
\mathrm{T}\left(\mathrm{n}_{\mathrm{e}}\right) & =1, & & \mathrm{n}_{\mathrm{e}}<\mathrm{n}_{\mathrm{cr}} \\
& =0, & & \mathrm{n}_{\mathrm{e}} \geq \mathrm{n}_{\mathrm{cr}}
\end{aligned}
$$

Total transmitted fluence through the substrate front surface is calculated from the integral below (see figure 4.2.2).

$$
F_{\text {transmitted }}=\int_{-\infty}^{\infty} I(t) T\left(n_{e}\right) d t=\int_{-\infty}^{t_{0}} I \sec h^{2}\left(\frac{1.762 t}{\tau}\right) d t
$$

where $t_{0}$ is the time at which electron density reaches critical value, $\tau$ is the FWHM pulsewidth of the laser pulse, and I is the peak laser intensity.

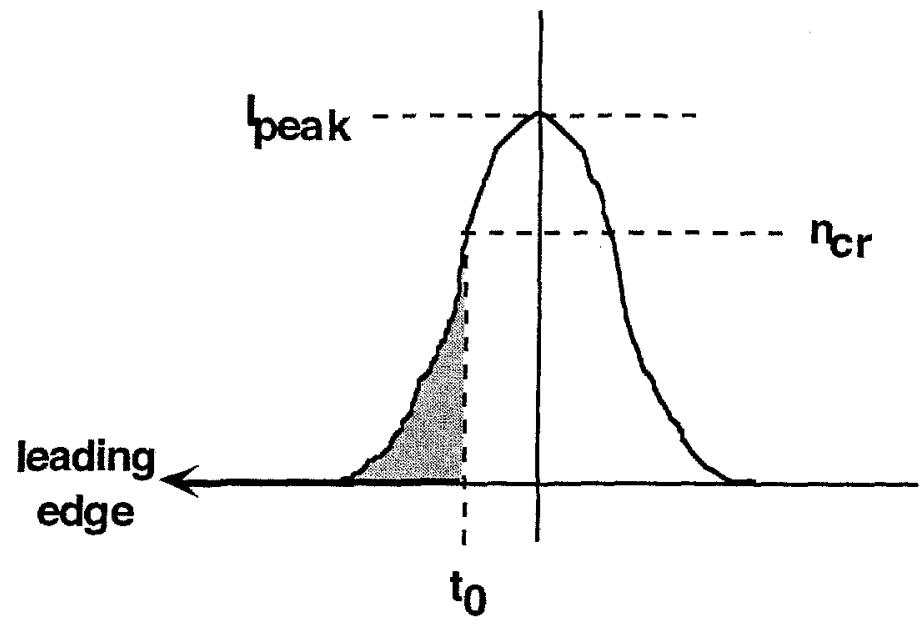

\section{Gray area=transmitted fluence}

Figure 4.2.2. Diagram showing transmitted fluence as the leading edge of the incident waveform

Solving (4.1) by the fourth-order Runge-Kutta method with a specific value of A produces a relation for $t_{0}$ as a function of intensity. By inserting $t_{0}$ into (4.3), we produce a relation for $F_{\text {transmitted }}$ as a function of incident fluence on the substrate front surface. We then integrate over the gaussian spatial pulse profile to obtain the total transmitted pulse energy as a function of incident pulse energy. Figures 3.2.2 and 3.2.3 show the 
experimentally measured ratio of transmitted laser energy and incident laser energy for 3.33 psec and 1.85 psec pulses transmitted through uncoated BK-7 substrate strips, as well as calculated values for this ratio generated by equations (4.1) and (4.2). We find a reasonable fit to the data with an A value of $0.0065 \mathrm{~cm}^{2} / \mathrm{psec} \mathrm{GW}$ for both laser pulsewidths.

In the case of coated $\mathrm{Al}$ targets, we directly compare $\mathrm{F}_{\text {transmited }}$ as a function of front surface fluence to the edge velocity data by including $F_{\text {transmitted }}$ in the edge velocity plots shown in figures 4.2.3-4.2.6. In each case, $\mathrm{A}$ is chosen such that the incident fluence threshold value, $J_{t r}$, at which $F_{\text {transmitted }}$ maximizes coincides with the observed transition fluence, $J_{t h}$. This choice is equivalent to selecting the $A$ value such the observed $J_{t h}$ is the minimum laser fluence necessary to produce a critical electron density at the end of the laser pulse. Solving (4.1) by the fourth-order Runge-Kutta method with specific values of $\mathrm{A}$ for both pulse durations $1.85 \mathrm{ps}$ and $3.33 \mathrm{ps}$ produces a relation for $\mathrm{t}_{0}$ as a function of intensity as shown in figure 4.2 .7 . These A values range from 0.005 to $0.013 \mathrm{~cm}^{2} / \mathrm{psec}$ GW and are in good agreement with the A values fitted to the uncoated BK-7 substrate case. These $\mathrm{A}$ values are strongly affected by $\mathrm{Al}$ target substrate roughness, and the range of values shown in A arise in large part due to uncertainty in substrate surface roughness. For comparison, theoretical A values for fused silica have been shown to range from 0.0065 to $0.013 \mathrm{~cm}^{2} / \mathrm{psec} \mathrm{GW}$ and are in good agreement with the range of calculated A values. It has been shown that calculated substrate damage fluence thresholds, which are defined as the minimum fluence necessary to produce critical electron density, of superpolished fused silica, are in agreement with the calculated threshold values of the unpolished BK-7 targets used in this experiment ${ }^{1}$. Clearly, self-induced laser ionization 
in the substrate is acting as a dynamic velocity-limiting mechanism for plumes emitted by LIBA.

An intriguing aspect of this laser-induced substrate effect is that for shorter laser pulses (below 1 ps), the fluence threshold for damage decreases ${ }^{2}$. This causes the total transmitted fluence to decrease dramatically. Since the ablation fluence threshold must be exceeded by the incident laser pulse, the substrate damage restricts the utility of laser pulses with pulsewidths below 1 ps.

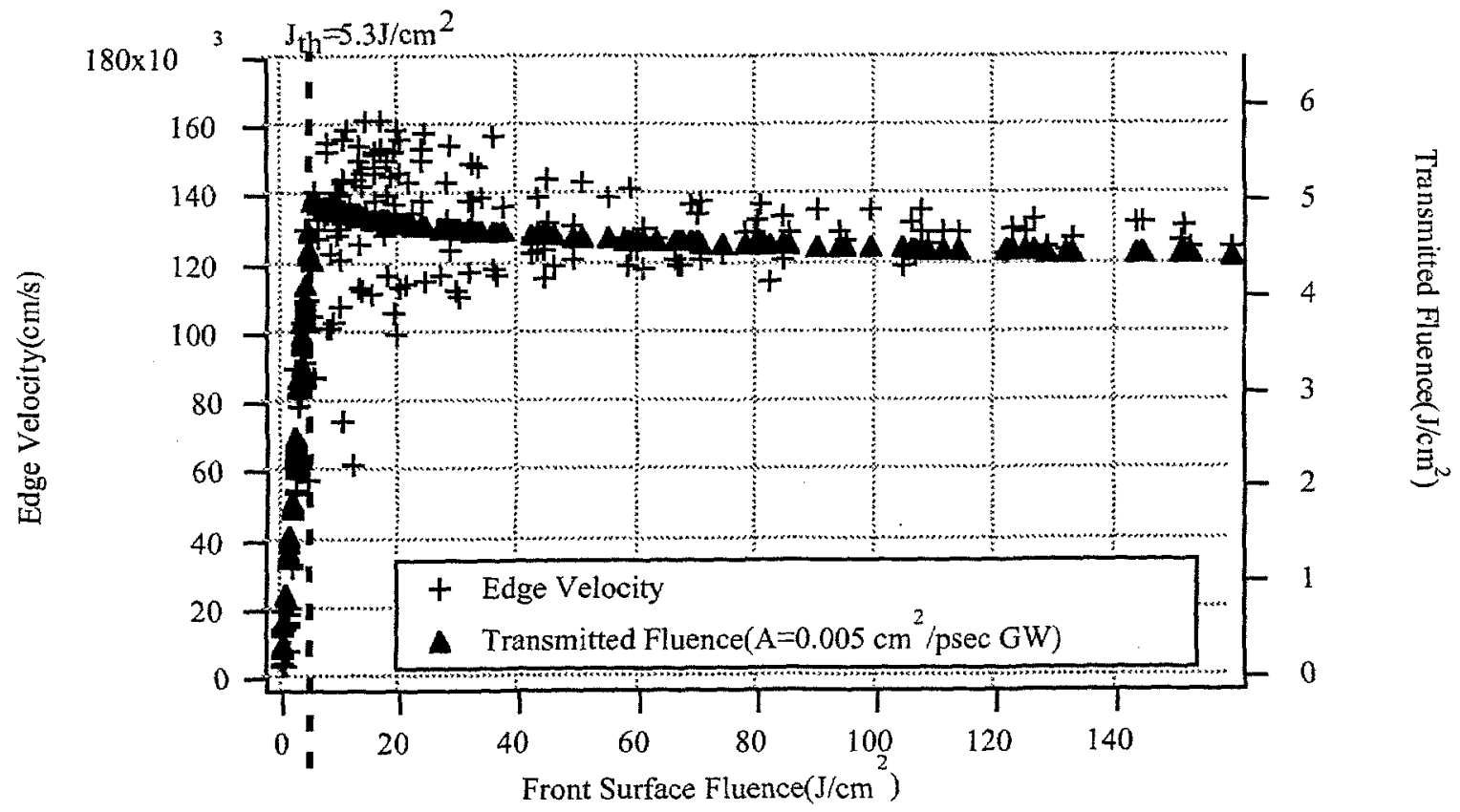

4.2.3. A plot of Al plume edge velocity as a function of incident front-surface fluence for $0.25 \mu \mathrm{m}$ films ablated by $1.85 \mathrm{psec}$ laser pulses is shown. Calculations of transmitted fluence as a function of front surface fluence are also shown. 


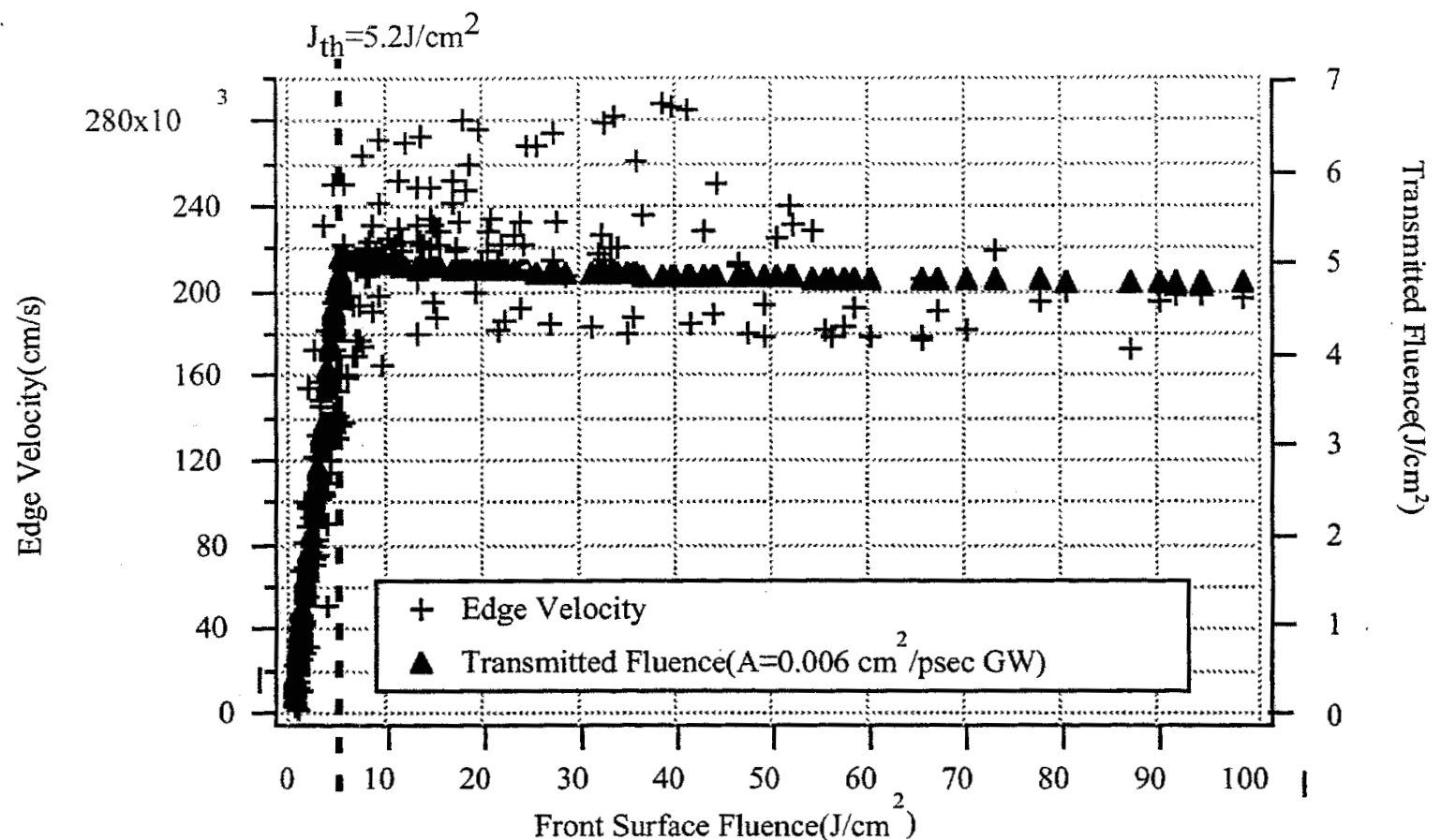

Figure 4.2.4. A plot of Al plume edge velocity as a function of incident front-surface fluence for $0.25 \mu \mathrm{m}$ films ablated by 3.33 psec laser pulses is shown. Calculations of

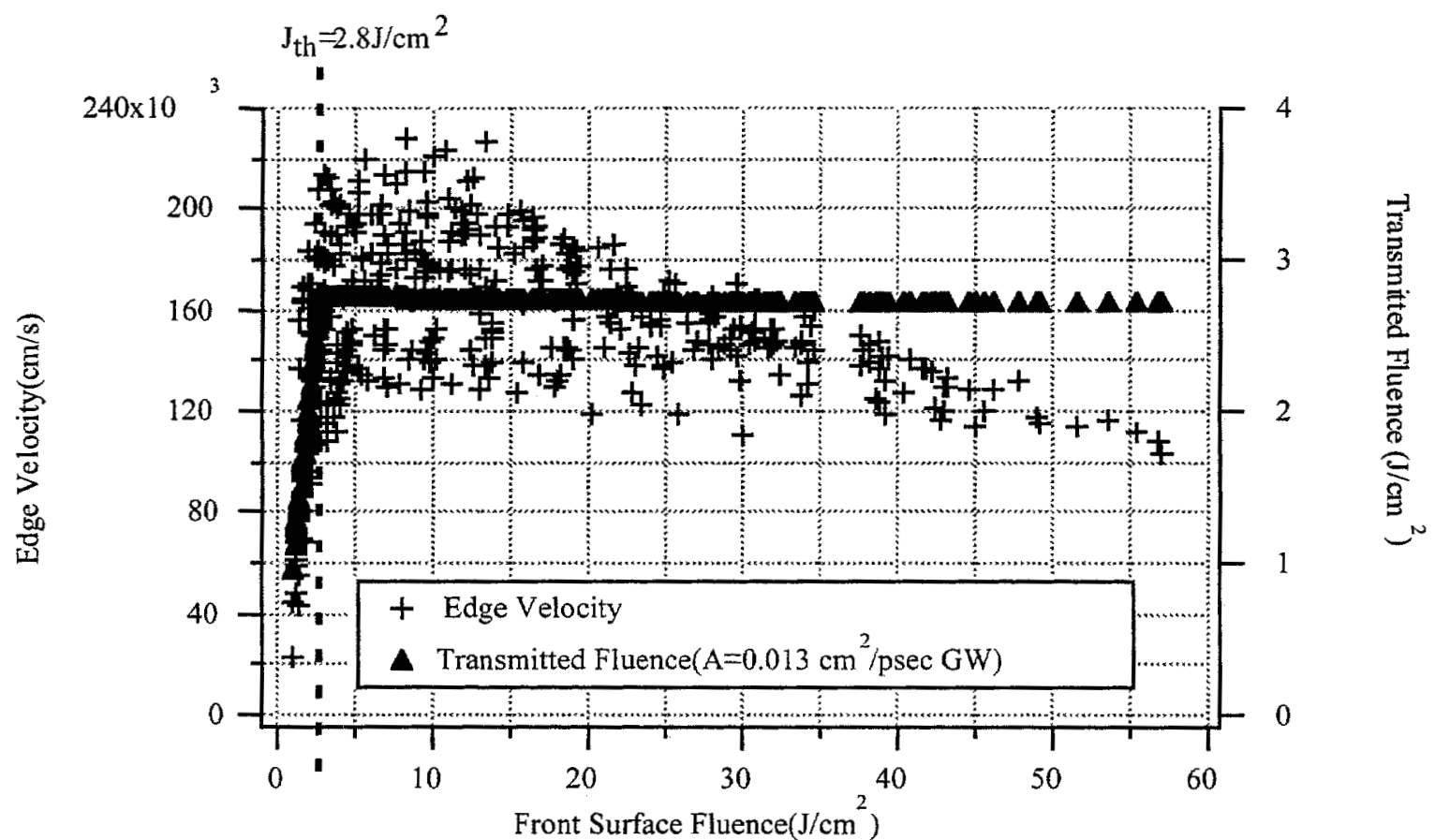

transmitted fluence as a function of front surface fluence are also shown.

Figure 4.2.5. A plot of Al plume edge velocity as a function of incident front-surface fluence for $0.50 \mu \mathrm{m}$ films ablated by $3.33 \mathrm{psec}$ laser pulses is shown. Calculations of transmitted fluence as a function of front surface fluence are also shown. 


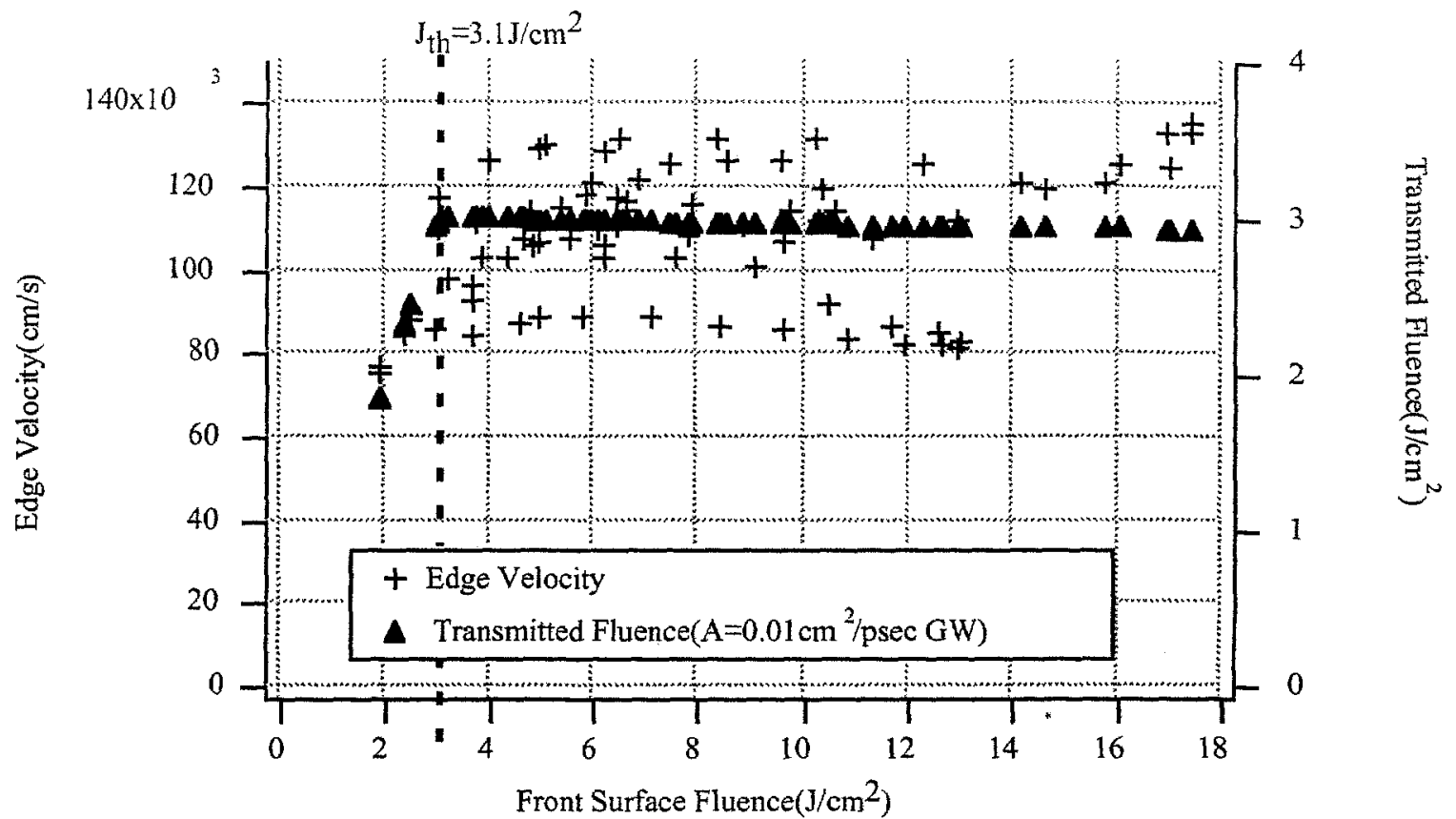

Figure 4.2.6. A plot of Al plume edge velocity as a function of incident front-surface fluence for $0.50 \mu \mathrm{m}$ films ablated by $1.85 \mathrm{psec}$ laser pulses is shown. Calculations of transmitted fluence as a function of front surface fluence are also shown.

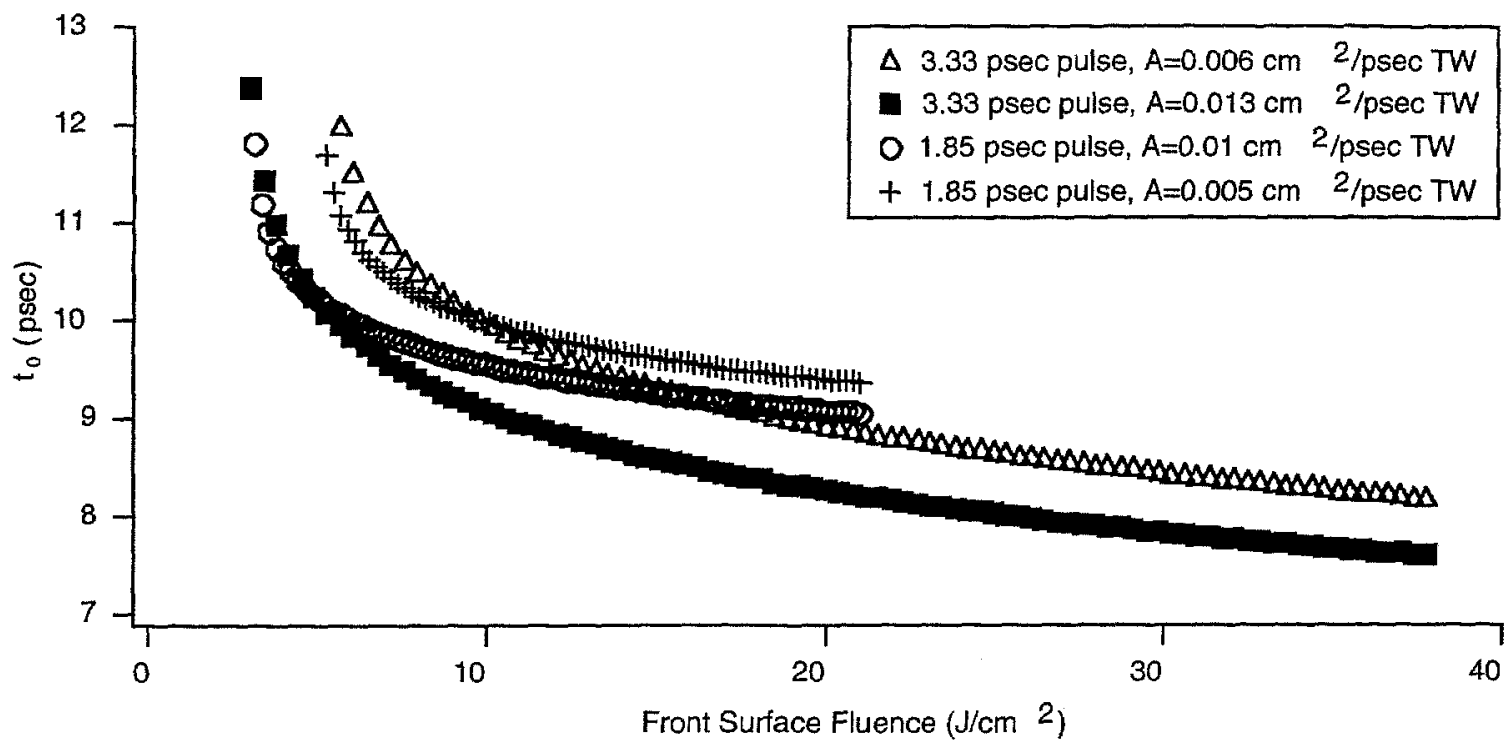

Figure 4.2.7. Graph of $t_{0}$ as a function of front surface fluence $\left(\mathrm{Sech}^{2}(\mathrm{t})\right.$ temporal profile, peak centered at $10 \mathrm{psec}$ ). 


\subsection{Laser Absorption in the Aluminum Thin Film}

When the leading edge of the ablation pulse transmits through the substrate and into the Al thin film, the thin film is a highly reflective solid $(\mathrm{R} \cong 92 \%)$. The film has a high conduction electron density $\left(\mathrm{n}_{\mathrm{e}} \cong 1.8 \times 10^{23} \mathrm{~cm}^{-3}\right)$, and the incident field is limited to a region of only a few skin depths $(\delta \cong 16.7 \mathrm{~nm})^{3}$ in thickness inside the film near the film/substrate interface. As the laser heats and ionizes the $\mathrm{Al}$ film, however, the resulting plasma can expands. This expansion can strongly affect the laser absorption in the expanding $\mathrm{Al}$ film, so to understand the laser/thin film absorption process, we must investigate any significant absorption changes caused by plasma expansion. By solving the Helmholtz equation for a finite scale-length plasma for a normally-incident laser pulse, we can calculate the absorption as a function of scale length. Then, by making estimates of the average plasma scale length during the laser/film interaction, we can evaluate the effect of plasma expansion on laser absorption.

We follow the Milchberg method ${ }^{4}$ for solving the 1-D Helmholtz wave equation shown below.

$$
\frac{d^{2} \tilde{E}}{d x^{2}}+k^{2} n^{2} \tilde{E}=0
$$

where $\mathrm{E}$ is the complex electric field, and $\mathrm{n}$ is the complex index of refraction.

We assume the atomic density distribution can be described by the linear function $N(x)=N_{0}(1-x / L)$ for $0 \leq x \leq L, N(x)=0$ for $x \geq L$, and $N(x)=N_{0}$ for $x=0$ (see figure 4.3.1). The

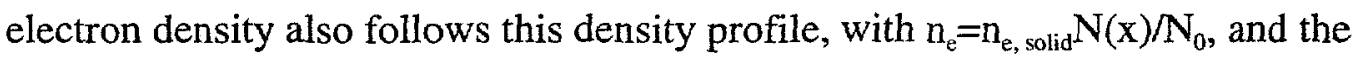
collisionality also is proportional to the neutral atom density $\left(v / \omega=v / \omega_{\text {solid }} N(x) / N_{0}\right)$. We then solve the Helmholtz equation for the reflectivity. We assume a collisionality value 
$\left(v / \omega_{0}=1 / 1.7\right)$ which, in the limit of very small scale lengths $(\mathrm{L}<<0.001 \lambda)$, results in a reflectivity value equal to solid $\mathrm{Al}$ reflectivity. Figure 4.3.2 shows the calculated reflectivity as a function of scale length, L. Assuming no off-axis scattering, absorption is also plotted of this graph as a function of scale length.

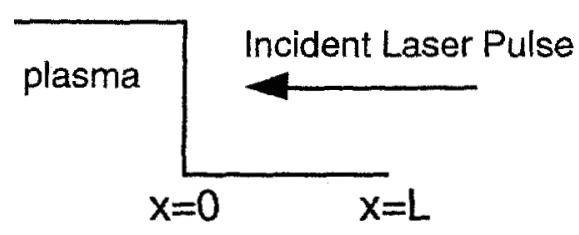

Initial plasma profile $(t=0)$

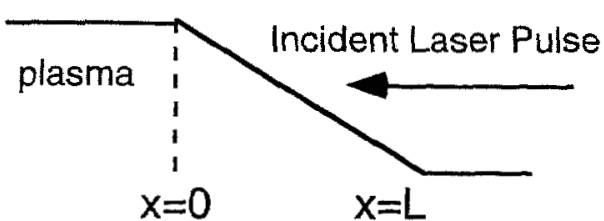

Expanding plasma profile

$(t>0)$

Figure 4.3.1. Plasma profiles showing the initial and expanding density profiles. The Helmholtz equation is solved using the "expanding plasma" density profile.

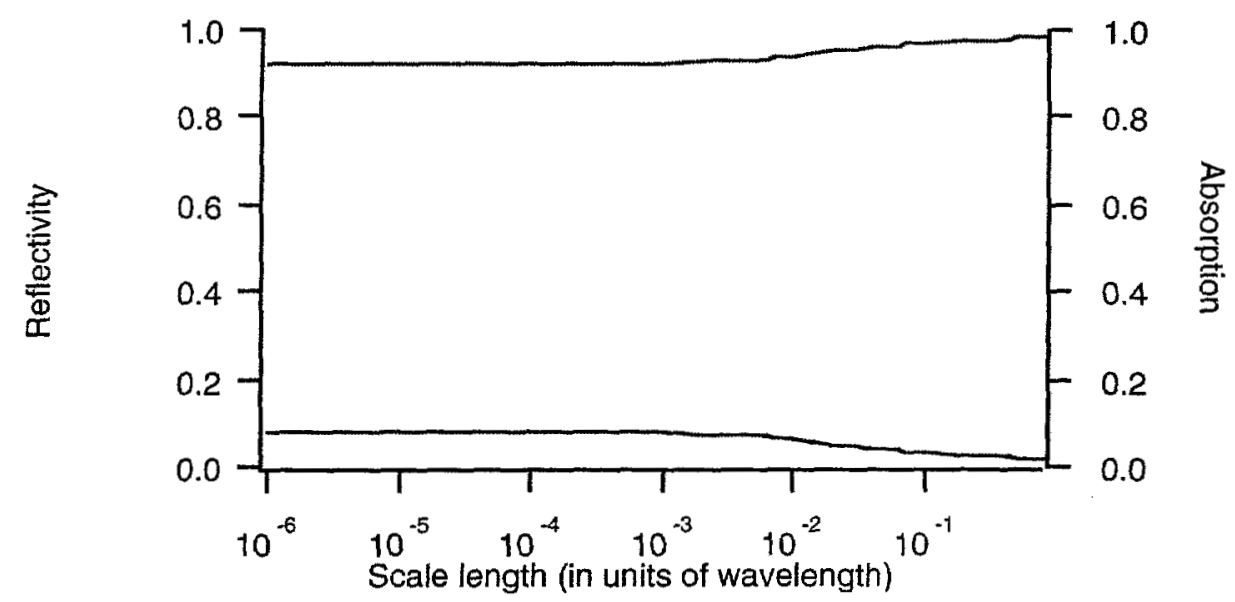

Figure 4.3.2. Plot of reflectivity and absorption as a function of scale length. The scale length axis is in units of wavelength $(1053 \mathrm{~nm})$. The reflectivity curve is the top curve, and the bottom curve is the absorption curve.

Maximum observed LIBA edge velocity is approximately $3 \times 10^{5} \mathrm{~cm} / \mathrm{s}$. If we assume plume expansion is constant after ablation, one would expect the Al plume to expand no more than $0.02 \lambda$ during the duration of the pulse. Even scale length expansion up to $0.02 \lambda$ during the duration of the pulse would cause minimal changes in reflectivity, so we would expect minimal deviations from the solid $\mathrm{Al}$ reflectivity due to plasma expansion. 


\subsection{HYADES Simulation of LIBA}

Although the calculation of transmitted fluence through a substrate shows that the saturation onset regime of the edge velocity coincides with the substrate damage regime, further calculations using hydrodynamics and energy transport theory are required to better understand the energy transfer process between the incident laser pulse and the ejected Al plume. We use HYADES, a 1-D Lagrangian hydrodynamics and energy transport code, to simulate LIBA. HYADES allows the user to model laser-driven ablation with user-specified laser intensity temporal pulse profiles. HYADES divides the aluminum film into 50 material layers or zones. As HYADES simulates the ablation event, it computes pressure, temperature, and density values for each zone at set time intervals, thereby providing temporal histories for these parameters.

We use HYADES to model the ablation event in the manner below ${ }^{5}$. The ablation event is modeled in a planar geometry. Electrons and ions are treated separately in a fluid approximation, and both are in thermodynamic equilibrium described by MaxwellBoltzmann statistics. The equations of state for both the aluminum thin film and substrate are provided by Sesame EOS tables ${ }^{6}$. We used the Thomas-Fermi ionization model to simulate ionization in the $\mathrm{Al}$ film and the Saha ionization model for the substrate. For ease of calculation, a substrate thickness of $45 \mu \mathrm{m}$ is used, and the substrate, assumed to be made up of entirely of $\mathrm{SiO}_{2}$, is divided into 100 zones. Although the substrate is actually $1.2 \mathrm{~mm}$ thick, the $45 \mu \mathrm{m}$ thickness is almost semi-infinite during simulation of 
the ablation event, and the physical difference between this thickness and $45 \mu \mathrm{m}$ is negligible.

The laser source injects energy directly into the Al film at the substrate/film interface. HYADES does not include a Helmholtz equation wave solver, and any laser source is absorbed into the $\mathrm{Al}$ film with approximately $100 \%$ absorption by inverse bremsstrahlung (errors in the calculation prevent $100 \%$ energy absorption). Since this absorption is too high (assuming $95.21 \%$ reflectivity, solid aluminum would be expected to absorb less than $5 \%$ of the incident energy), the actual input laser source intensity profile must be scaled by the expected laser-energy-transfer-efficiency (LETE) so that the Al film absorbs the correct amount of laser energy. LETE is the fraction of the laser energy which is converted into absorbed energy in the HYADES calculation, and as such, LETE is a measure of laser energy absorbed by not only direct Al/laser interaction but also any laser/substrate interactions near the substrate/film interface which can contribute to the ablation mechanism. Also, since the substrate breakdown effect limits the transmitted fluence through the substrate, the input laser source profile must be truncated at $t_{0}$, as defined in the substrate damage model.

\section{HYADES Simulation of Edge Velocity}

We used HYADES simulations to model the observed edge velocity. Since the atomic density of the first material zone is higher than the minimum visible density observed in the shadowgraphs, we compare the velocity of the first zone with the edge velocity. Using laser pulses with peak intensities well above the substrate damage breakdown intensity (i.e. asymptotic regime), we use the model to output edge velocity values at $t=10 \mathrm{~ns}$ and $\mathrm{t}=2 \mathrm{~ns}$. Here we define $\mathrm{t}=0$ to be $10 \mathrm{ps}$ before the peak of the 
incident laser pulse. Since the calculated velocity of the first Al zone does not change between $2 \mathrm{~ns}(>600 \tau$, where $\tau$ is the laser pulsewidth) and $10 \mathrm{~ns}(>3000 \tau)$, we treat the edge velocity at these long times as constant. We can then compare calculated first zone velocity after $2 \mathrm{~ns}$ of simulated time with the observed edge velocities at $255 \mathrm{~ns}$ after the ablation event. Since the laser energy absorption of the $\mathrm{Al}$ film has not been experimentally measured, we use the LETE coefficient as an adjustable parameter in these simulations. Figure 4.4.1 shows a plot of the calculated velocity of the first zone as a function of LETE. This dependence is similar to a square root function of incident fluence. This functional form is consistent with the self-similar expression of LIBA edge velocity during an adiabatic decompression, $\mathrm{v}_{\text {edge }}=\left(6 \mathrm{~T}_{0} / \mathrm{m}_{\mathrm{i}}\right)^{1 / 2}$, produced by Mayer and Busch $^{7}$. Here, $T_{0}$ is the initial temperature of the plume prior to expansion and is proportional to the incident fluence. In the case shown in figure 4.4.1, the HYADES velocity will match the experimental observed edge velocity when LETE is $0.085 \pm 0.005$. Using this LETE value, we calculate the 1st zone velocity over the full range of front surface fluence. These calculated values are shown in figure 4.4.2. 


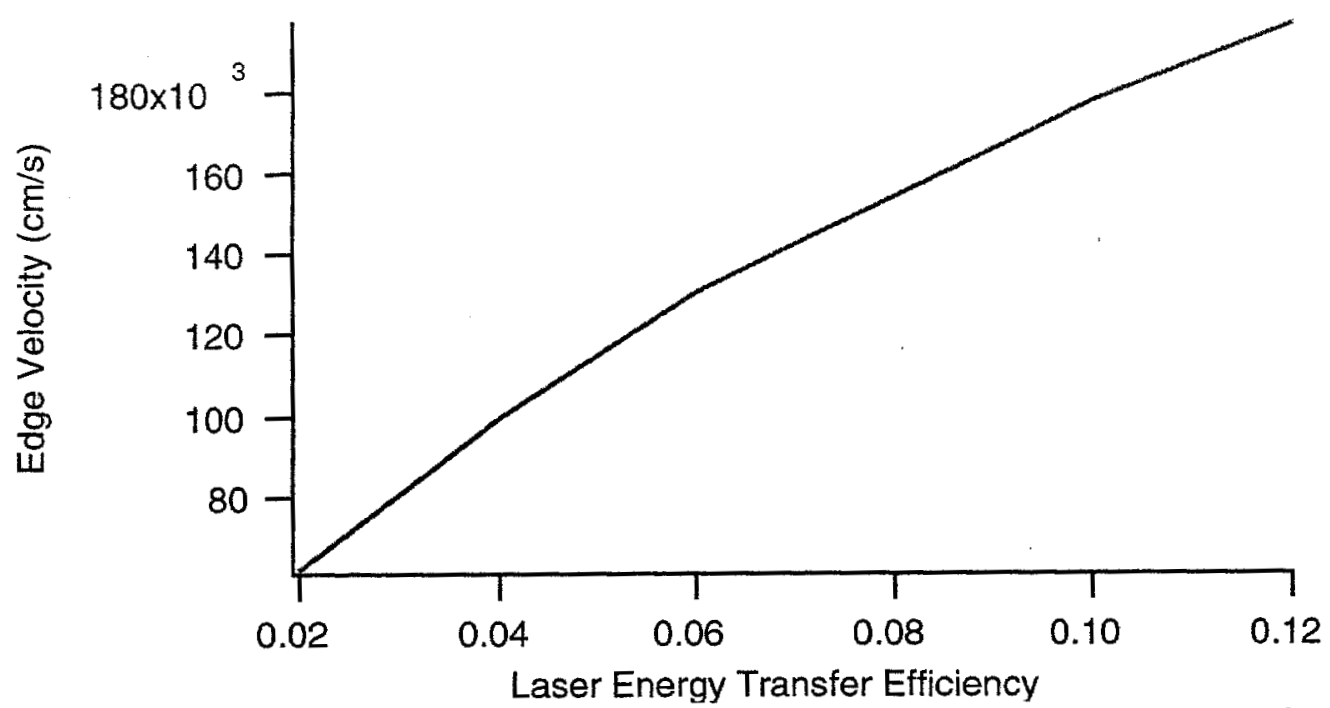

4.4.1. Plot of calculated velocity of the first zone of a $0.5 \mu \mathrm{m} \mathrm{Al} \mathrm{film} 2 \mathrm{~ns}$ after interaction with a $3.33 \mathrm{ps}$ ablation pulse (peak front surface fluence $9.72 \mathrm{~J} / \mathrm{cm}^{2}$ )

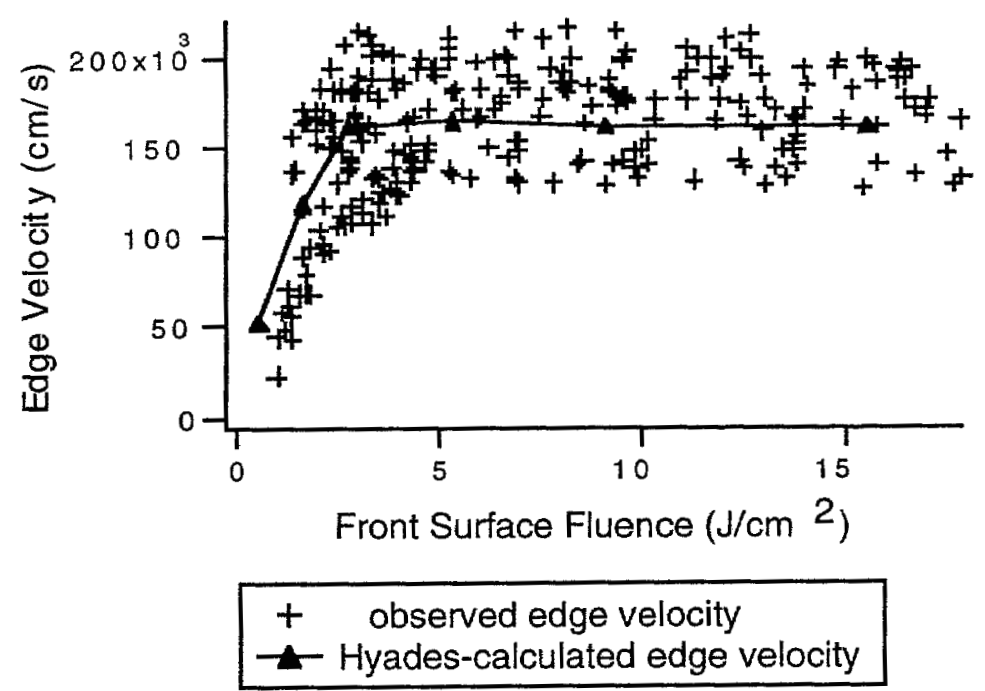

Figure 4.4.2. Plot of observed edge velocity and calculated edge velocity as a function of front surface fluence for a $0.5 \mu \mathrm{m} \mathrm{Al}$ film ablated by a 3.33 ps pulse. The HYADEScalculated edge velocity uses a LETE value of 0.085 .

Similar calculations for the $0.25 \mu \mathrm{m}, 3.33$ ps case show that a LETE value of $0.085 \pm 0.005$ yields the calculated velocity which matches the data. These calculated 


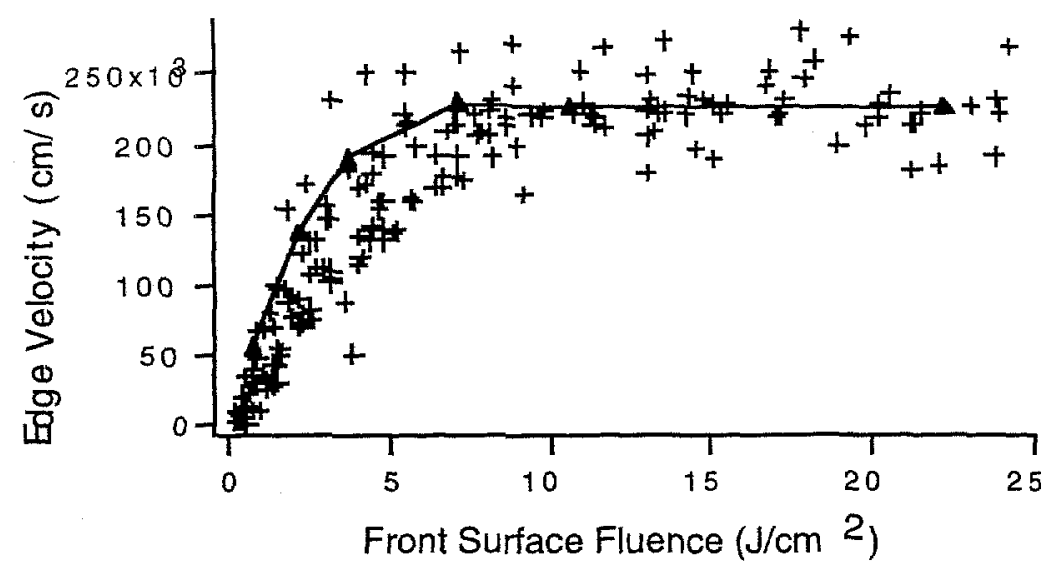

\section{$+\quad$ observed edge velocity \\ - Hyades-calculated edge velocity}

Figure 4.4.3. Plot of observed edge velocity and calculated edge velocity as a function of front surface fluence for a $0.25 \mu \mathrm{m} \mathrm{Al}$ film ablated by a 3.33 ps pulse.

values are shown in 4.4.3. The LETE values for both the $0.25 \mu \mathrm{m}, 3.33$ ps case and the $0.5 \mu \mathrm{m}, 3.33$ ps case are higher than estimated absorption value, $A=1-R$, provided by solid aluminum reflectivity ${ }^{8}$. The reflectivity at $1053 \mathrm{~nm}, \mathrm{R}=0.9521$, yieids an estimated absorption value, A, of 0.0479 .

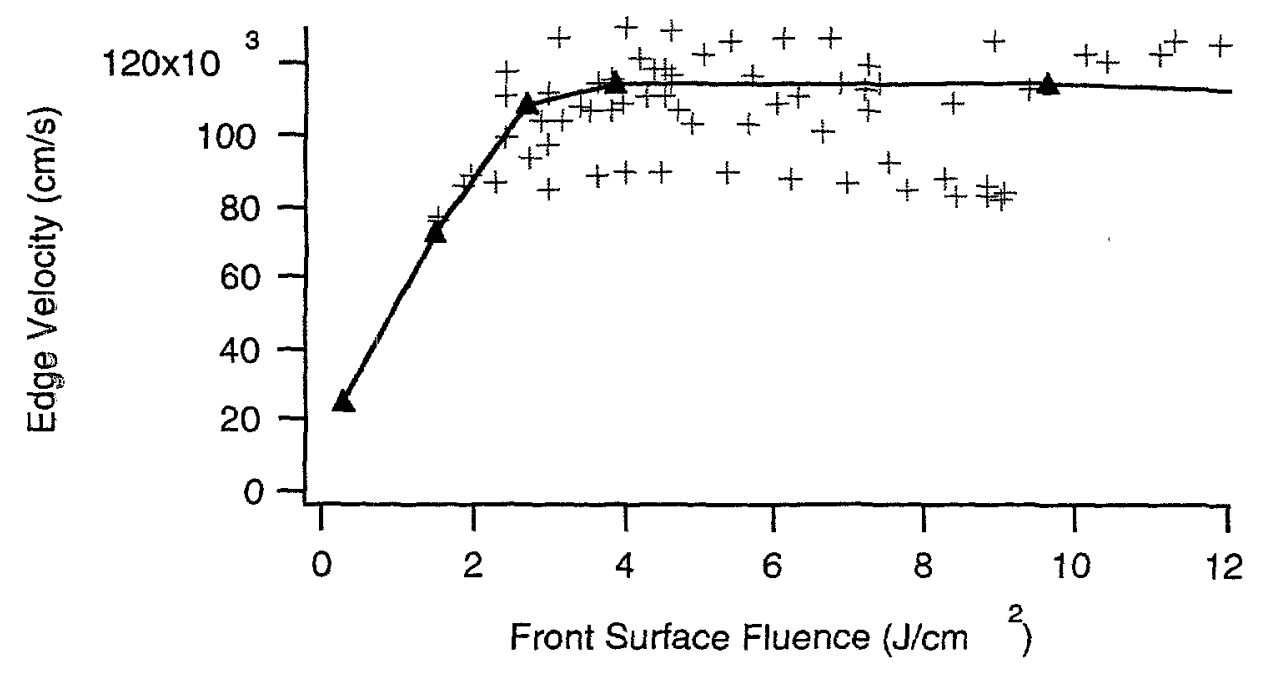

+ observed edge velocity calculated edge velocity 
Figure 4.4.4. Plot of observed edge velocity and calculated edge velocity as a function of front surface fluence for a $0.25 \mu \mathrm{m} \mathrm{Al} \mathrm{film} \mathrm{ablated} \mathrm{by} \mathrm{a} 1.85 \mathrm{ps}$ pulse.

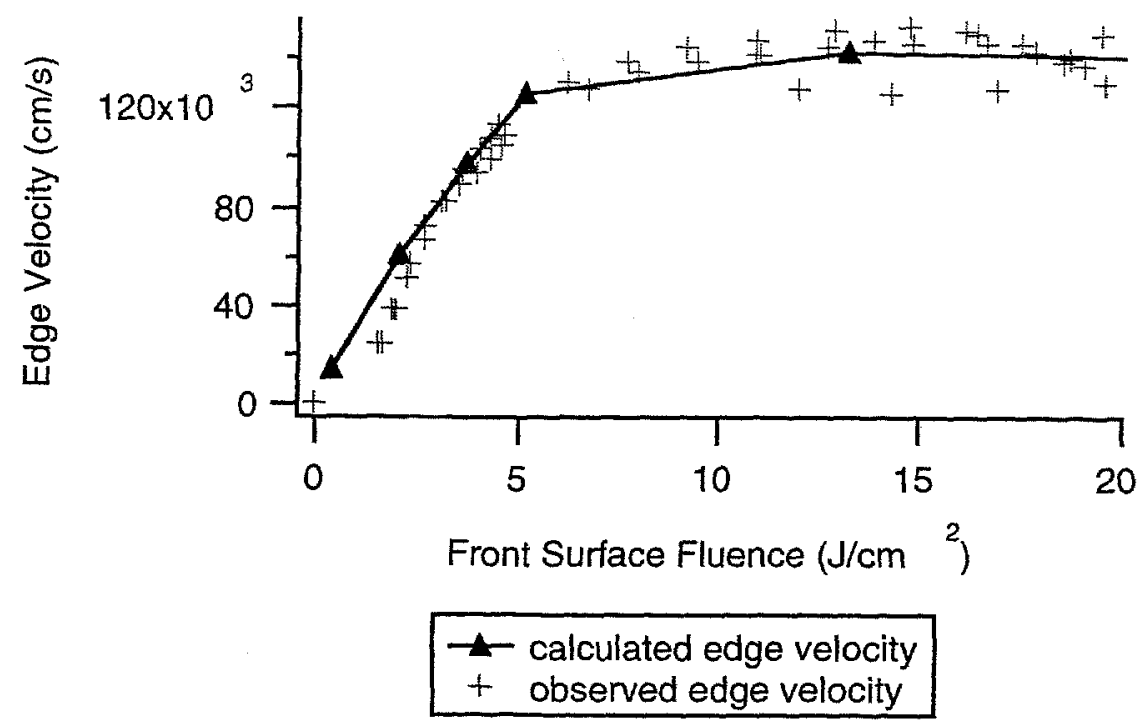

Figure 4.4.5. Plot of observed edge velocity and calculated edge velocity as a function of front surface fluence for a $0.50 \mu \mathrm{m} \mathrm{Al}$ film ablated by a 1.85 ps pulse.

Figure 4.4.4 shows both the observed and calculated edge velocities for the $0.25 \mu \mathrm{m}, 1.85$ ps case. In this case, a LETE value of $0.031 \pm 0.005$ fits the calculated velocities to the data. In Figure 4.4 .5 , the $0.50 \mu \mathrm{m}, 1.85 \mathrm{ps}$ case is shown, and the LETE value used is $0.044 \pm 0.005$. Both cases involving 1.85 ps pulses had LETE values closer to the solid $\mathrm{Al}$ absorption value than the $3.33 \mathrm{ps}$ cases. This suggests that the Al thin film absorption rises dramatically during the lifetime of the 3.33 ps pulse, while the 1.85 ps pulses are too short for absorption to increase noticeably during the pulse duration.

As mentioned earlier, the low fluence edge velocity behavior shows the existence of a LIBA fluence threshold. It is interesting to note that in figures 4.4.2-4.4.5, the HYADES-calculated edge velocities do not show this threshold behavior. Even fluences below the observed ablation fluence threshold will result in a non-zero first zone velocity in HYADES simulations. This is caused by the fluid approximation used in HYADES. 
The Al film is not treated as a solid, with cohesive and adhesive forces bonding the thin film to itself and to the substrate, and therefore there is no adhesive force in HYADES acting against the pressure produced by the ablation pulse. This difference between HYADES and the actual experiment suggests that the threshold effect is caused by adhesion and cohesion in the solid.

\section{Interface Density Behavior}

One of the more important aspects of LIBA is the evolution of density in time. Since, experimentally, it is very difficult to measure the density in early time, we use HYADES to calculate the interface density. Figure 4.4.6 shows calculated density of the Al material zone initially at the substrate/aluminum interface as a function of time for three different ablation pulses. For each plot, thickness of the film is divided by the expansion time required to drop the zone density to half solid density. This value is then the expansion velocity for the interface zone (zone 50). The (A) plot shows a very low expansion velocity $\left(\mathrm{v}<10^{4} \mathrm{~cm} / \mathrm{s}\right)$. The (B) plot has a much higher expansion velocity $\left(\mathrm{V} \approx 10^{5} \mathrm{~cm} / \mathrm{s}\right)$. The (C) plot shows the expansion velocity is approximately equal to that in the (B) plot. Since both calculations involve the same amount of total absorbed fluence in the $\mathrm{Al}$ film, this suggests that expansion velocity, like edge velocity, is strongly affected by the laser-induced substrate damage. 


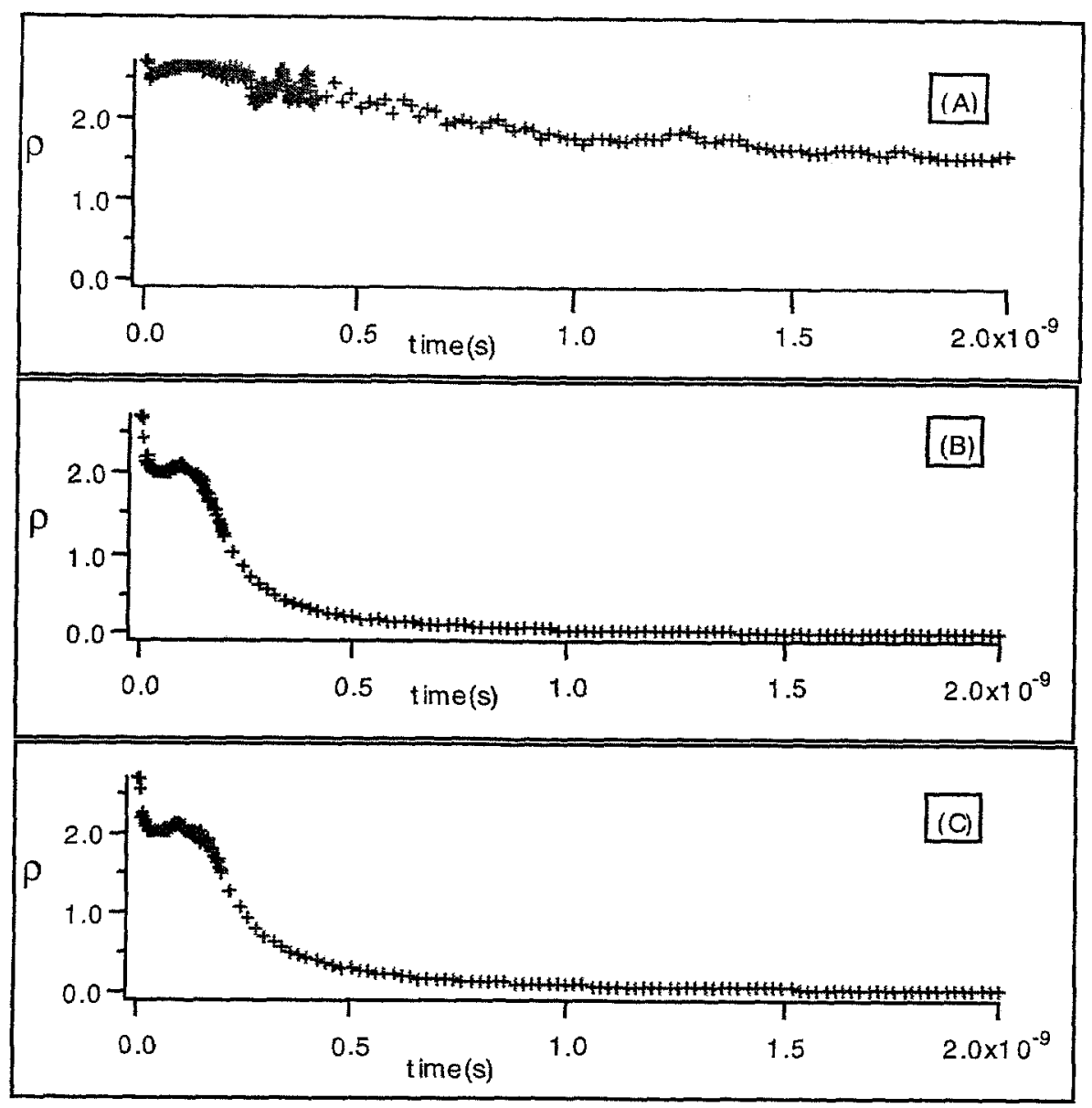

Figure 4.4.6. Plot of HYADES-calculated density, $\rho\left(\mathrm{g} / \mathrm{cm}^{3}\right)$, of interface zone (zone 50) as a function of time for plumes generated by $0.25 \mu \mathrm{m} \mathrm{Al} \mathrm{films} \mathrm{ablated} \mathrm{by} 3.33$ ps pulses. Plot (A) corresponds to a laser pulse with $0.79 \mathrm{~J} / \mathrm{cm}^{2}$ peak front surface fluence. Plot (B) corresponds to a laser pulse with $7.18 \mathrm{~J} / \mathrm{cm}^{2}$ peak front surface fluence. Plot (C) corresponds to a laser pulse with $22.3 \mathrm{~J} / \mathrm{cm}^{2}$ peak front surface fluence.

Similar behavior is seen in figure 4.4 .7 for the $0.5 \mu \mathrm{m}, 3.33$ ps case. The two plumes, (B) and $(C)$, both exhibit the same interface expansion velocity $\left(5.4 \times 10^{4} \mathrm{~cm} / \mathrm{s}\right)$, which further indicates that substrate damage strongly affects expansion velocity. As in figure 4.4.6, the lower fluence case in figure 4.4 .7 (A) shows a very low expansion velocity $\left(\mathrm{v}<<10^{4}\right.$ 


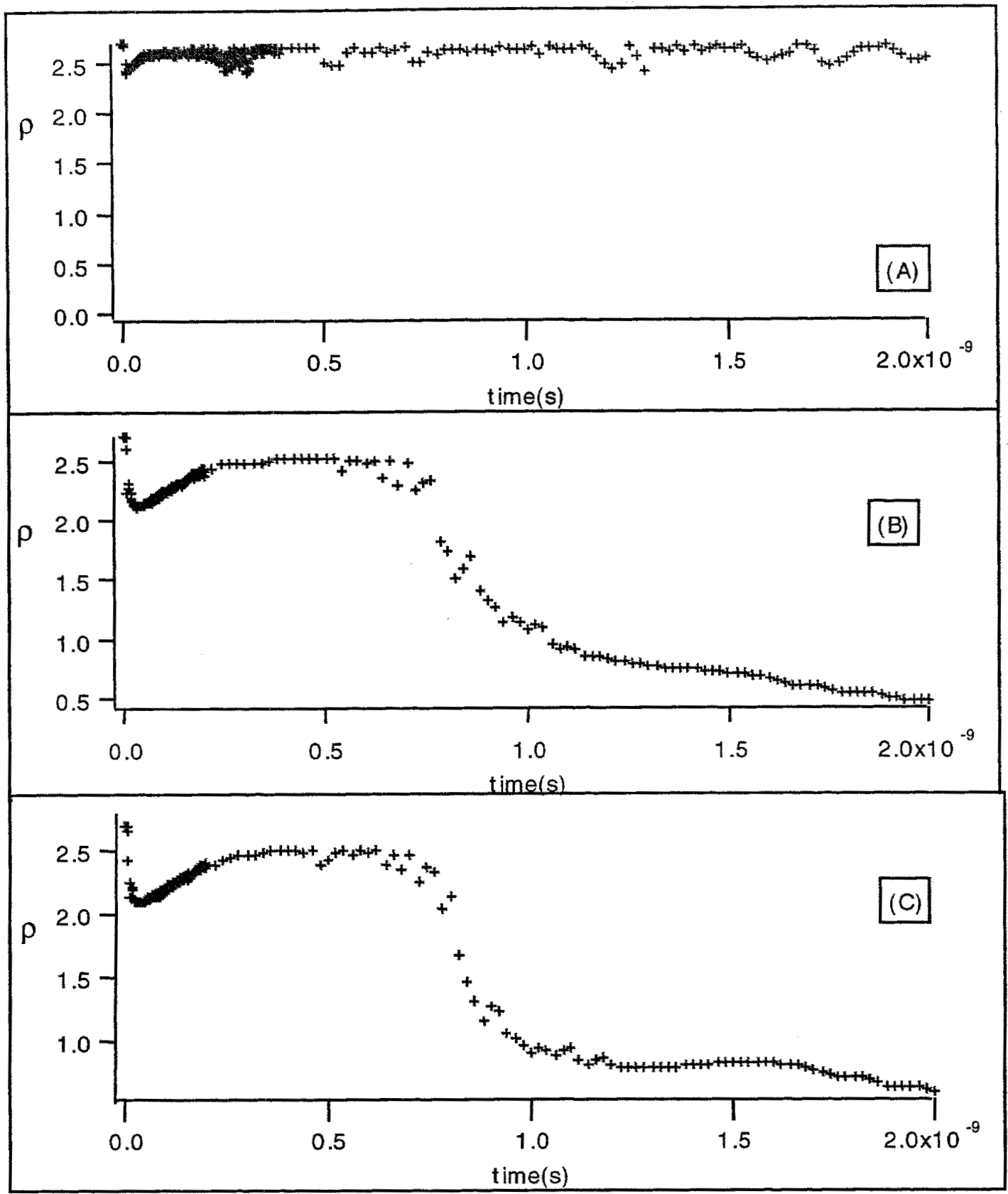

Figure 4.4.7. Plot of HYADES-calculated density, $\rho\left(\mathrm{g} / \mathrm{cm}^{3}\right)$, of interface zone (zone 50) as a function of time for plumes generated by $0.5 \mu \mathrm{m} \mathrm{Al}$ films ablated by $3.33 \mathrm{ps}$ pulses. Plot (A) corresponds to a laser pulse with $0.8 \mathrm{~J} / \mathrm{cm}^{2}$ peak front surface fluence. Plot (B) corresponds to a laser pulse with $5.38 \mathrm{~J} / \mathrm{cm}^{2}$ peak front surface fluence. Plot (C) corresponds to a laser pulse with $15.7 \mathrm{~J} / \mathrm{cm}^{2}$ peak front surface fluence. 

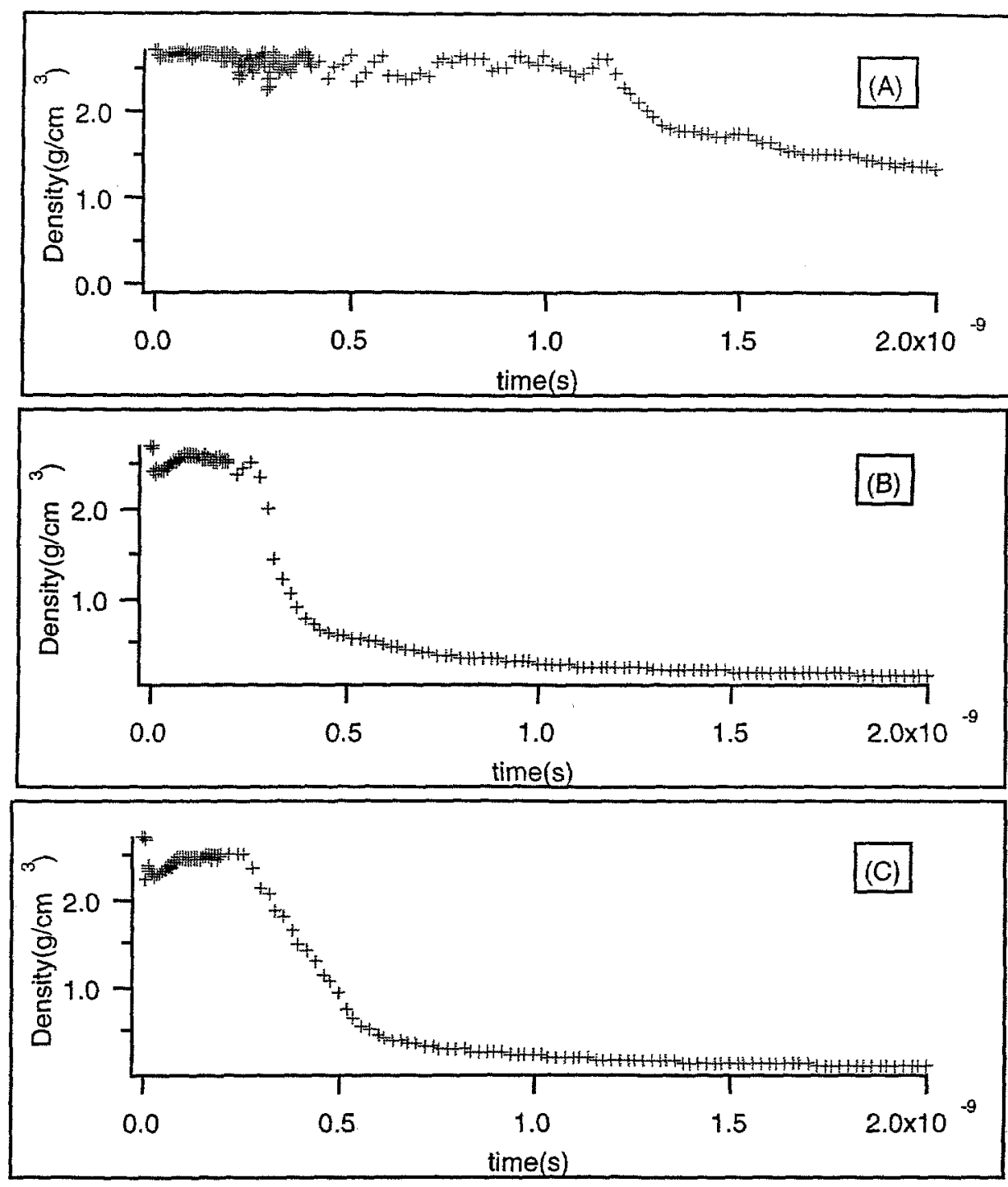

Figure 4.4.8. Plot of HYADES-calculated density as a function of time for plumes generated by $0.25 \mu \mathrm{m} \mathrm{Al}$ films ablated by 1.85 ps pulses. Plot (A) corresponds to a laser pulse with $0.918 \mathrm{~J} / \mathrm{cm}^{2}$ peak front surface fluence. Plot (B) corresponds to a laser pulse with $3.6 \mathrm{~J} / \mathrm{cm}^{2}$ peak front surface fluence. Plot (C) corresponds to a laser pulse with 12.8 $\mathrm{J} / \mathrm{cm}^{2}$ peak front surface fluence.

$\mathrm{cm} / \mathrm{s}$ ). In both figures 4.4.6 and 4.4.7, we see, for cases of similar front surface fluence, that the expansion velocity of the interface zone is less than that of the edge velocities observed experimentally later in time. 
This indicates that the expansion of the interface is limited by the inertia of the outer Al zones, and the outer zones are still accelerating outwards. Figure 4.4.8 shows the calculated density for $0.25 \mu \mathrm{m}$ films ablated by 1.85 ps pulses. Both (B) and (C) show approximately the same expansion velocity $\left(6.0 \times 10^{4} \mathrm{~cm} / \mathrm{s}\right)$, and this expansion velocity is lower than that for the 3.33 ps case. This is attributed to the low LETE value (0.031) used to match the observed edge velocity data, and it shows that both interface expansion velocity and calculated edge velocity show a similar dependence on LETE.

\section{Interface Pressure Behavior}

Also important is the relationship between interface pressure and interface expansion. Comparing pressure as a function of time with density as a function of time allows us to understand ablation dynamics. Of particular importance is the interface pressure history, since this layer directly receives the laser energy and, in the limit of very short pulses of very high fluence, would form an high-pressure explosive layer which detaches the rest of the film and forces it away from the substrate as a projectile (the "flyer plate" concept). Figure 4.4.9 shows the interface pressure (zone 50) as a function of time for plumes produced by ablating $0.25 \mu \mathrm{m}$ films with $3.33 \mathrm{ps}$ pulses. In this figure, the peak of the pulse is timed to occur $10 \mathrm{ps}$ after the start of the simulation. The pressure 
peaks in very early time ( $t \approx 3 \tau$, where $\tau$ is the laser pulsewidth) for all three plots and then relaxes to a low pressure in times $\mathrm{t}<30 \tau$. Expansion does not reduce the interface density to half solid value until much later in time, however $(t>100 \tau)$. This indicates that heat is diffusing into the thickness of the film before substantial expansion occurs, and cooling is the important pressure reduction mechanism in very early times. In the "flyer plate" concept described previously, expansion occurs on a time scale that precludes substantial heat diffusion away from the interface region. The pressure relaxation rate should be equal to the expansion rate, and expansion should occur on a time scale short enough to preclude heat diffusion. This is not the case in picosecond pulse LIBA. Figure 4.4.6 and figure 4.4.9 show, at the interface (zone 50), density and pressure drop at very different rates. The drop in pressure in early time shows the heat diffusion rate is faster than the expansion rate. We must then conclude that the "flyer plate" concept does not adequately describe the ablation process by picosecond laser pulses. Also of interest is the pressure build-up time. The pressure build-up time does not change noticeably for large increases in peak pressure, which suggests that pressure build-up should follow the temporal laser profile. The peak fluence used to calculate the pressure shown in plot 4.4.9(A) is equal to the threshold fluence for ablation for a $0.25 \mu \mathrm{m}$ Al film ablated by 3.33 ps pulse $\left(0.79 \mathrm{~J} / \mathrm{cm}^{2}\right)$. 


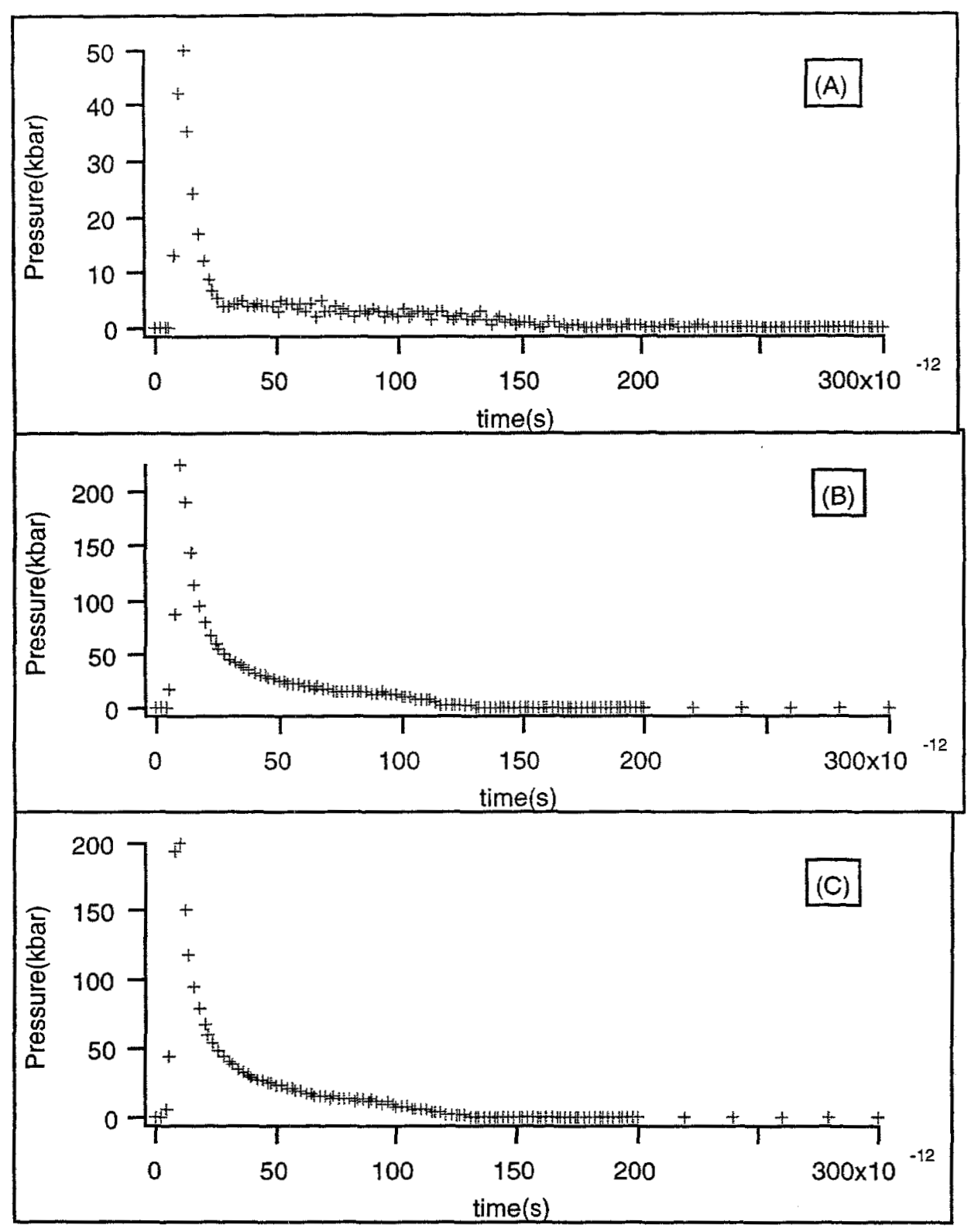

Figure 4.4.9. Plot of HYADES-calculated pressure as a function of time for plumes generated by $0.25 \mu \mathrm{m} \mathrm{Al}$ films ablated by 3.33 ps pulses. Plot (A) corresponds to a laser pulse with $0.79 \mathrm{~J} / \mathrm{cm}^{2}$ peak front surface fluence (ablation threshold fluence). Plot (B) corresponds to a laser pulse with $7.18 \mathrm{~J} / \mathrm{cm}^{2}$ peak front surface fluence. Plot (C) corresponds to a laser pulse with $22.3 \mathrm{~J} / \mathrm{cm}^{2}$ peak front surface fluence.

In this case, the peak pressure value shown in $4.4 .9(\mathrm{~A})$ has special significance. In HYADES simulations in very early time $(t<100 \mathrm{ps})$, the expansion of the Al film is minimal, and the interface zone maintains an approximate solid density. This behavior is similar to that of the actual solid Al film, and at these early times, the fluid approximation 
is not expected to effect the accuracy of HYADES pressure calculations. In this case, the peak pressure shown in figure 4.4.9(A) would equate to the threshold pressure ( $P \approx 50$ kbars) required to overcome the adhesive and cohesive bonds responsible for the ablation fluence threshold. Similarly, the peak pressures shown in figure 4.4 .10 (A) and (B) are equal to the threshold pressure for ablation.

To better understand this threshold pressure, the peak adhesion force between a unit area of $\mathrm{Al}$ film and the substrate is calculated. Each $\mathrm{Al}$ atom at the metal/substrate

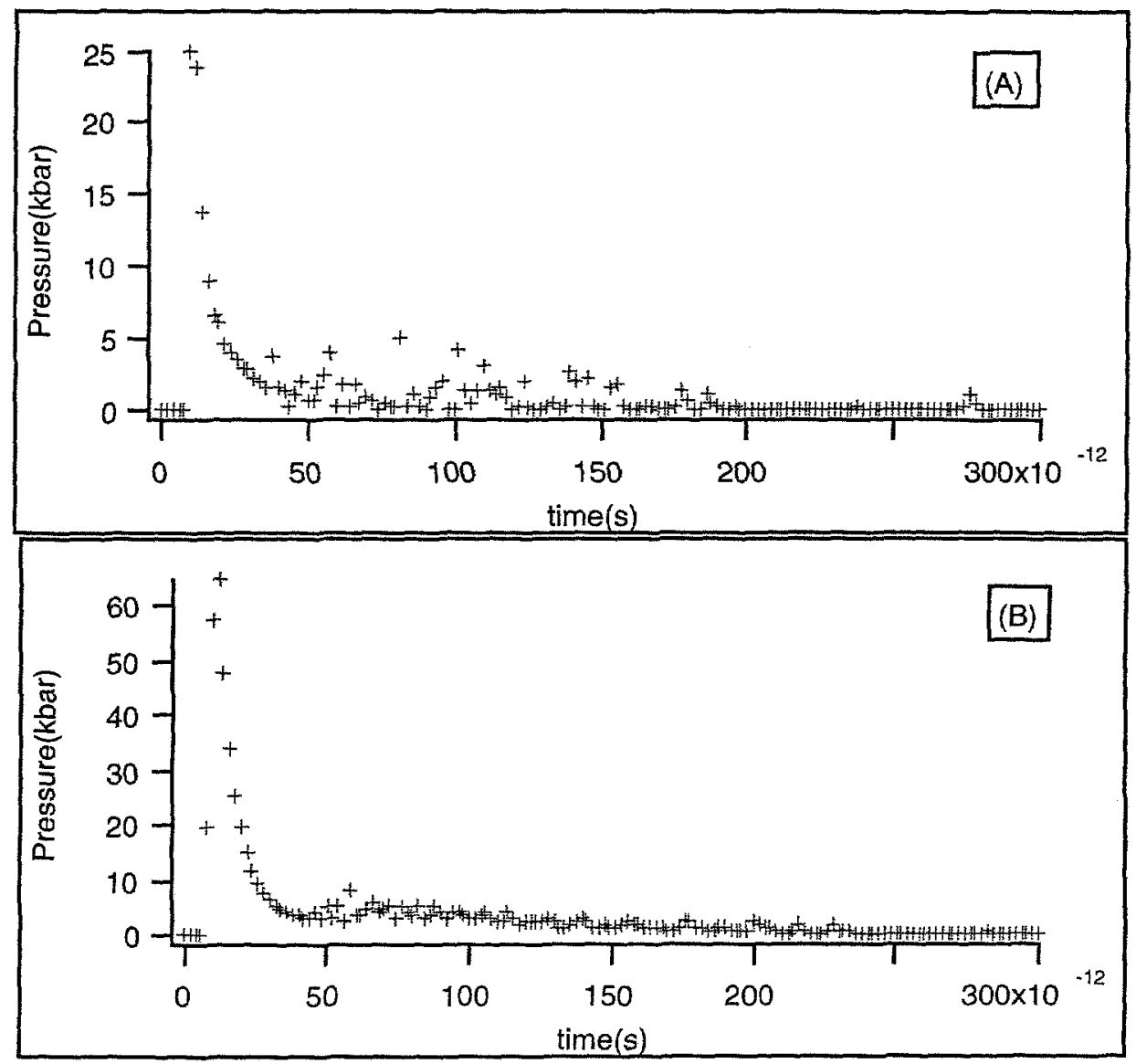

Figure 4.4.10. Plot of interface pressure as a function of time. Plot (A) corresponds a plume generated by ablating a $0.25 \mu \mathrm{m} \mathrm{Al} \mathrm{film} \mathrm{with} \mathrm{a} 1.85 \mathrm{ps}$ pulse $\left(0.918 \mathrm{~J} / \mathrm{cm}^{2}\right.$ peak front surface fluence) (ablation threshold fluence). Plot (B) corresponds a plume generated by ablating a $0.5 \mu \mathrm{m}$ Al film with a 3.33 ps pulse $\left(0.8 \mathrm{~J} / \mathrm{cm}^{2}\right.$ peak front surface fluence). 
interface is assumed to be bonded to the substrate. Also, each aluminum-to-substrate bond is expressed as a Van der Waals bond with $0.286 \mathrm{~nm}(2 \times \mathrm{Al} \text { atomic radius })^{9}$ between the bonded atoms. Based on these assumptions, the peak adhesion force per unit area would be 424 kbars. Comparing this value to the threshold pressure suggests that, at the thin film/substrate interface of the $\mathrm{Al}$ target, less than $17 \%$ of $\mathrm{Al}$ atoms at the interface are bonded to the substrate.

\subsection{Plume Temperature Analysis}

Plume temperature is a fundamental quantity directly connected to many important aspects of the LIBA process, and understanding $\mathrm{Al}$ plume temperature is important in understanding LIBA dynamics. We use both experimental techniques, calculations, and HYADES simulations to understand plume temperature. During the LIBA experimental study, we measured the observed divergence in Al plumes at multiple time delays. We also recorded the clipped optical ablation spectra for LIBA-generated plumes. In this section, by using the SAHA ionization model, we relate the relative line intensities for these clipped spectra to an time-averaged and spatially integrated plume temperature. We compare this temperature to the divergence-based estimates of maximum plume temperature discussed in a previous section. We also compare these values to HYADES calculations of plume temperature in early time.

The utility of this approach is shown below. The divergence estimates originating from the shadowgraphs indicate plume temperature at late times (of order hundreds of nanoseconds). The HYADES simulations provide a temporally-resolved, spatially resolved 1-D temperature profile, and HYADES produces temperatures in both very early 
time $(\mathrm{t}<10 \mathrm{ps})$ and late time $(\mathrm{t}>2 \mathrm{~ns})$. The spectral temperature estimates are timeintegrated over $33 \mathrm{~ms}$, but PMT data of the ablation event show the strong ablation emissions have a duration of less than $50 \mathrm{~ns}$. The spectral temperature estimates are

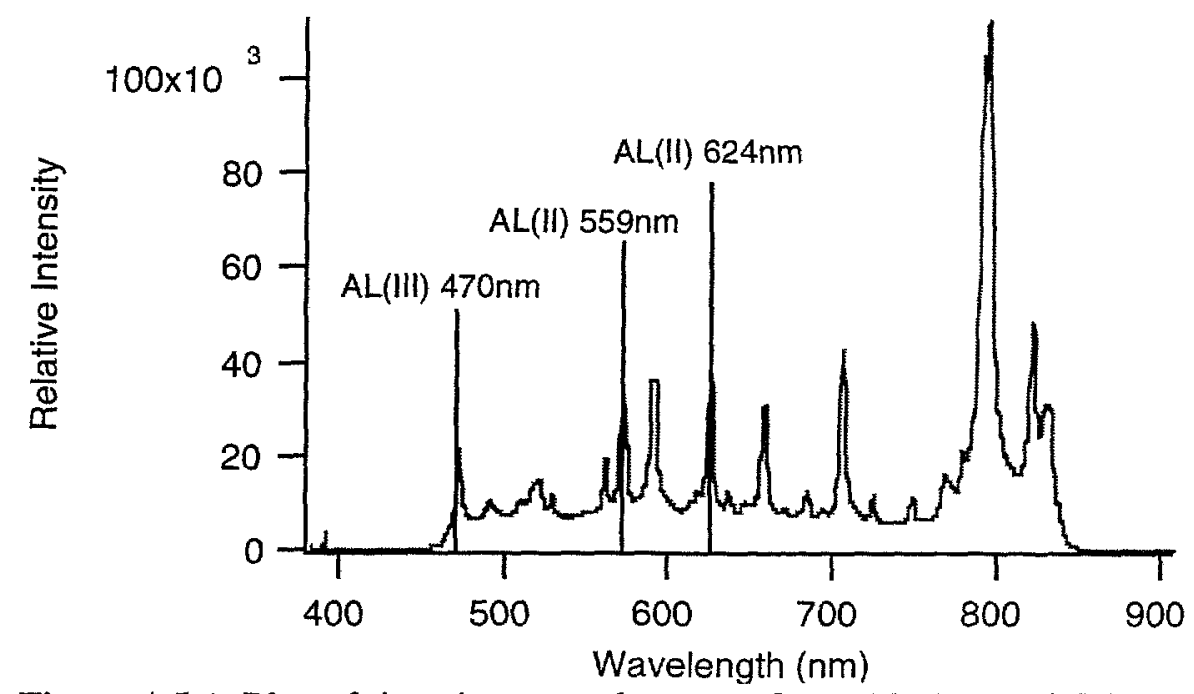

Figure 4.5.1. Plot of time-integrated spectra from Al plume $(0.25 \mu \mathrm{m}$ film, a $3.33 \mathrm{ps}$ pulse, $12.3 \mathrm{~J} / \mathrm{cm}^{2}$ peak front surface fluence)

therefore biased by the strong ablation-induced emission early in time. Together, these temperature estimates allow the experimenter to understand the ablation event in both very early and very late in time.

As shown in previous sections, ablating Al film with a clipped geometry produces a line-rich optical spectrum with a suppressed black body background. Figure 4.5.1 shows the spectra emitted from a Al plume generated by a $0.25 \mu \mathrm{m}$ film ablated by a 3.33 ps pulse. Three prominent $\mathrm{Al}$ lines, $\mathrm{Al} \mathrm{In}(470 \mathrm{~nm}), \mathrm{Al}$ I $(559 \mathrm{~nm})$, and $\mathrm{Al}$ II $(624 \mathrm{~nm})$, are each clearly identified as originating from a single transition. The Al III line originates

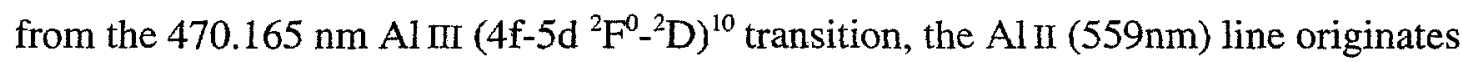
from the $559.323 \mathrm{~nm} \mathrm{Al} \mathrm{II}\left(3 \mathrm{~s} 5 \mathrm{p}-3 \mathrm{~s}\left({ }^{2} \mathrm{~S}\right) 7 \mathrm{~d}^{3} \mathrm{P}^{0}{ }_{-}^{3} \mathrm{D}\right){ }^{11}$ transition, and the Al II (624nm) line originates from the $623.74 \mathrm{~nm}$ Al $\pi\left(3 \mathrm{~s} 5 \mathrm{p}-3 \mathrm{~s}\left({ }^{2} \mathrm{~S}\right) 7 \mathrm{~d}^{3} \mathrm{P}^{0}-{ }_{-}^{3} \mathrm{D}\right)$ transition. The contribution of 
Si lines to the observed intensity of these lines can be safely ignored since spectra of laser/substrate interactions of similar pulsewidth, intensity, and fluence do not show these spectral lines. Since each line is clearly separated from the blackbody, we can relate the ratio of line intensities between each transition to an time-averaged, spatially-averaged electron temperature inside the plume emission region. In cases where the electrons and the neutral atoms are in thermal equilibrium with each other, this temperature becomes the temperature of the $\mathrm{Al}$ plume in that region.

We approach the line intensity ratios by applying the Saha ionization model to the problem. In cases of local thermal equilibrium (LTE), the relative populations of bound electrons at each atomic energy level can be described by the expression below ${ }^{12}$.

$$
\frac{N_{m}}{N_{n}}=\frac{g_{m}}{g_{n}} \exp \left[\frac{-\chi_{m, n}}{k T}\right]
$$

Where $\mathrm{N}_{\mathrm{m}}$ and $\mathrm{N}_{\mathrm{n}}$ are the atomic population levels at the upper energy level and lower energy level of the transition, respectively, $g_{m}$ and $g_{n}$ are the degeneracies for the upper and lower energy levels, respectfully, and $\chi_{m, n}$ is the energy difference between the upper and lower levels.

For free electrons and atoms, the population densities follow this relation.

$$
\frac{N(Z)_{0} N}{N(Z-1)_{0}}=2 \frac{g(Z)_{0}}{g(Z-1)_{0}}\left(\frac{m k T}{2 \pi \hbar^{2}}\right)^{\frac{3}{2}} \exp \left[\frac{-\chi(Z-1)_{0}}{k T}\right]
$$

Where $N(Z)_{0}$ and $N(Z-1)_{0}$ are the ground state populations for the $Z$ th ionization state and $(Z-1)$ th ionization state, respectively, $N$ is the electron density, $g(Z)_{0}$ and $g(Z-1)_{0}$ are the degeneracies for each state, and $\chi(Z-1)_{0}$ is the ionization energy for atoms of charge $(Z-1)$ in the ground state.

The total emissivity of a spectral line is described by the following:

$$
\varepsilon=4 \pi^{2} \hbar c^{2} r_{0}\left(\frac{g_{n}}{g_{m}}\right)\left(\frac{f_{m n}}{\lambda_{o}^{3}}\right) N_{m}
$$

Where $f_{m n}$ is the absorption oscillator strength for the transition. 
The ratio of two emitted spectral lines can then be described by the expression below.

$$
\frac{\varepsilon_{1}}{\varepsilon_{2}}=\frac{f_{m n}(1) g_{n}(1)}{f_{m n}(2) g_{n}(2)}\left(\frac{\lambda_{0}(2)}{\lambda_{0}(1)}\right)^{3} \exp -\left[\frac{E_{m}(1)-E_{m}(2)}{k T}\right]
$$

Now, if the two lines which make up the intensity ratio originate from different ionization states, the ratio changes to the following:

$$
\frac{\varepsilon_{1}}{\varepsilon_{2}}=\frac{f_{m n}(1) g_{n}(1)}{f_{m n}(2) g_{n}(2)}\left(\frac{\lambda_{0}(2)}{\lambda_{0}(1)}\right)^{3}\left(\frac{k T}{\chi_{H}}\right)^{\frac{3}{2}} \frac{\exp -\left[\frac{E_{m}(1)-E_{m}(2)+\chi-\Delta \chi}{k T}\right]}{4 \pi^{\frac{3}{2}} a_{0}^{3} N}
$$

Where $\chi$ is the ionization energy for the lower ionization state and $\Delta \chi$ is the ionization potential depression.

Here, the energy difference in the exponential includes not only the absolute energy level difference between the two upper states (which includes the ionization energy of the lower state) but also an ionization potential depression term, $\Delta \chi$. This term corresponds to the reduction of the ionization potential due to the presence of free electrons around the atoms and ions ${ }^{13}$.

Based on the expression above, the temperature is known if the electron density and relative line ratios are known. However, this expression is only valid under conditions of local thermal equilibrium (LTE). LTE requires that the collisional excitation and deexcitation processes dominate over the radiative processes in the ionization and excitation rate equations which describe the system. In this case, the system relaxes into the Maxwell-Boltzmann description shown above. These conditions are satisfied if the electron density is above a certain minimum density defined by the following ${ }^{14}$ :

$$
N \geq 1.4 \times 10^{14} T^{\frac{1}{2}} \chi_{m n}^{3}
$$


Where $\mathrm{T}$ and $\chi$ are in $\mathrm{eV}$.

In this experiment, PMT measurements show spectral emission to be limited to timescale less than $50 \mathrm{~ns}$ after the ablation pulse. This restricts the plume expansion to a maximum of $100 \mu \mathrm{m}$, and assuming a linear expansion, this would produce an average density (at $50 \mathrm{~ns}$ ) of order $10^{20} \mathrm{~cm}^{-3}$. Using a temperature $\mathrm{T}=1 \mathrm{eV}$ and an energy level separation of $\chi=5 \mathrm{eV}$, in excess of the spectral lines of interest, we find the minimum LTE electron density to be $1.75 \times 10^{16} \mathrm{~cm}^{-3}$. This would equate to a minimum ionization level of 0.0001 . Since the $0.1 \mu \mathrm{m}$ films show an ionization level an order of magnitude higher at $255 \mathrm{~ns}$, it is reasonable to expect these $\mathrm{Al}$ plumes to satisfy LTE criteria during the $50 \mathrm{~ns}$ emission timeframe.

Using the Saha ionization model, we calculate the average temperature. We assume an average electron density, $\mathrm{n}_{\mathrm{e}}$, of $1 \times 10^{18} \mathrm{~cm}^{-3}$, although the calculated temperature is only logarithmically dependent on $\mathrm{N}$. We also use the line ratios $\varepsilon_{470 \mathrm{~nm}} / \varepsilon_{624 \mathrm{~nm}}$ and $\varepsilon_{470 \mathrm{~nm}} / \varepsilon_{559 \mathrm{~nm}}$, since both ratios compare lines from species with different ionization states. For plumes generated by $0.25 \mu \mathrm{m}$ films ablated by 3.33 ps ablation pulses, we calculate temperature as a function of peak front surface fluence (see figure 4.5.2). Note that all the data points are in the substrate damage fluence regime, and it is very reasonable to expect the average plume temperature to be saturated with respect to increases in fluence. Also, the temperatures produced by the two line ratios are in excellent agreement. Similar results are found for plumes generated by $0.5 \mu \mathrm{m}$ films ablated with 1.85 ps pulses(see figure 4.5.3). Interestingly enough, similar results are also found in the $0.1 \mu \mathrm{m}$ film, 3.33ps case. Based on the substrate damage model and $\mathrm{Al}$ plume mass and assuming complete heat diffusion through the entire film thickness prior 
to ablation, the $\mathrm{Al}$ plumes would be expected to exhibit a different temperature in each case. In early time, however, the hot Al plume emission region is limited to an area near the substrate/thin film interface. Since the clipped geometry allows this region to radiate directly into the optics, and the ablated plume divergence in later time shows the plume to be too cold to radiate optical spectral lines, this data suggests that a hot core region of similar size in both $0.1 \mu \mathrm{m}$ and the $0.25 \mu \mathrm{m}$ plumes is responsible for the observed line spectra.

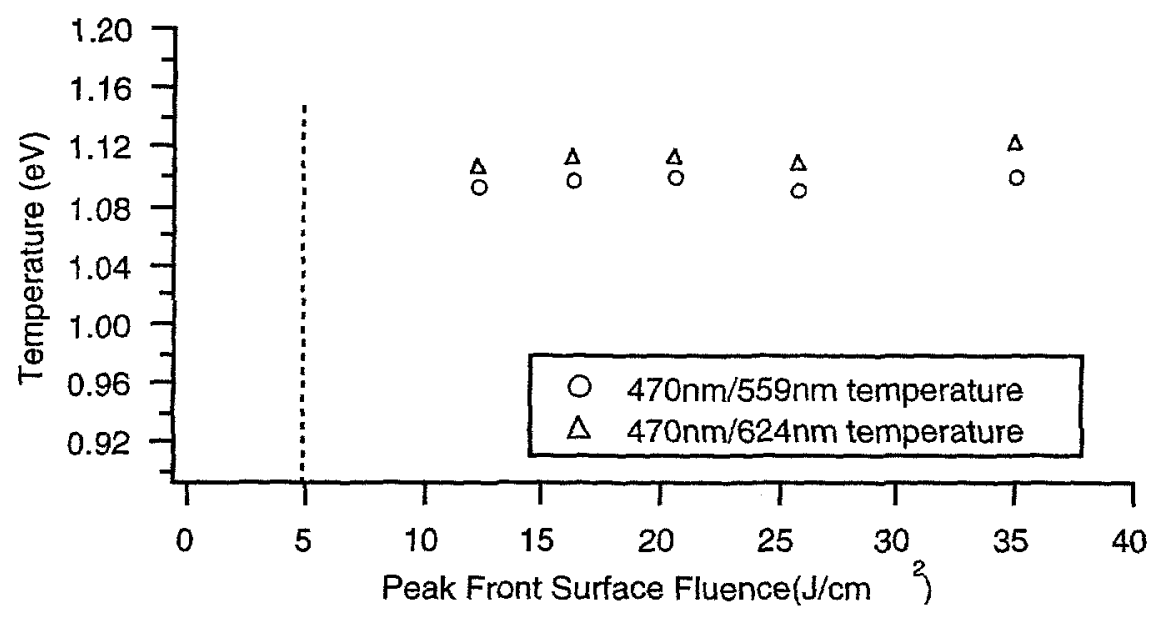

Figure 4.5.2. Plot of calculated temperature based on relative line ratios $470 \mathrm{~nm} / 559 \mathrm{~nm}$ and $470 \mathrm{~nm} / 624 \mathrm{~nm}$ for plumes generated by $0.25 \mu \mathrm{m}$ Al films ablated by 3.33 ps pulses. The dotted line identifies the substrate damage fluence threshold. 


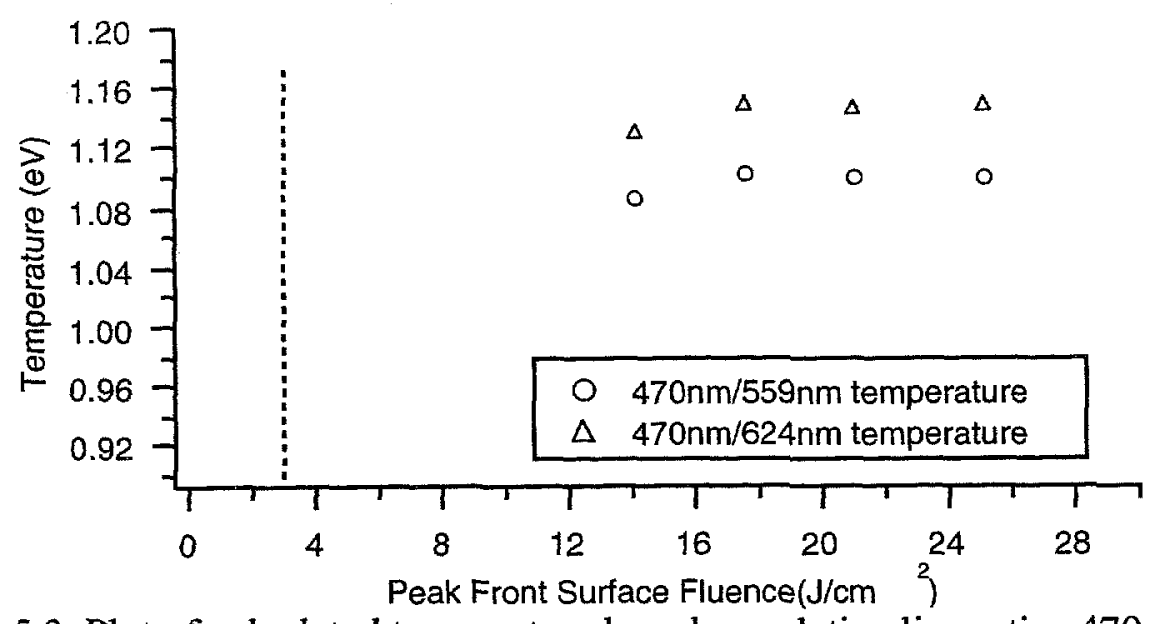

Figure 4.5.3. Plot of calculated temperature based on relative line ratios $470 \mathrm{~nm} / 559 \mathrm{~nm}$ and $470 \mathrm{~nm} / 624 \mathrm{~nm}$ for plumes generated by $0.5 \mu \mathrm{m} \mathrm{Al}$ films ablated by 1.85 ps pulses. The dotted line identifies the substrate damage fluence threshold.

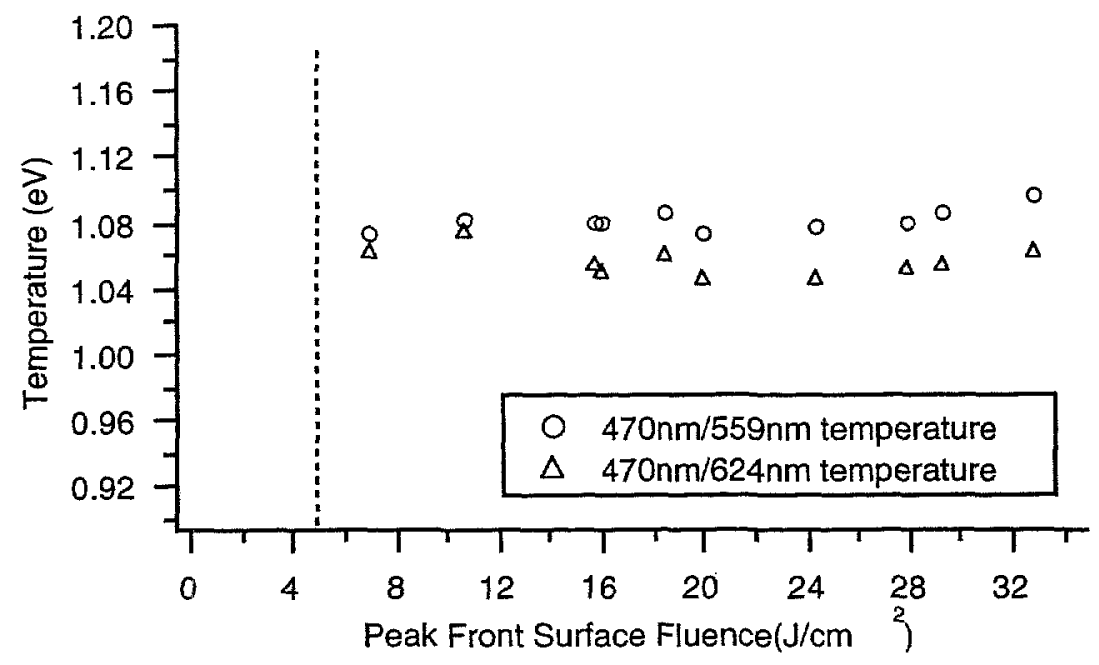

Figure 4.5.4. Plot of calculated temperature based on relative line ratios $470 \mathrm{~nm} / 559 \mathrm{~nm}$ and $470 \mathrm{~nm} / 624 \mathrm{~nm}$ for plumes generated by $0.1 \mu \mathrm{m} \mathrm{Al}$ films ablated by 3.33 ps pulses. The dotted line identifies the substrate damage fluence threshold.

In comparison to the calculated time-average temperatures shown above, HYADES calculations of the plume temperature in very early time show peak temperatures also in the $1 \mathrm{eV}$ range for fluence values well above $\mathrm{J}_{\mathrm{th}}$. Figure 4.5 .5 shows 
the interface temperature of $\mathrm{Al}$ plumes produced by $0.25 \mu \mathrm{m}$ films ablated by $3.33 \mathrm{ps}$ laser pulses. The temperature peaks at very early time $(\approx 10 \mathrm{ps})$ and decays quickly to a slowly decaying temperature $(<100 \mathrm{psec})$. This suggests that the lifetime of the hightemperature line emission time period is less than 100 ps and confirms that the integrated spectral data is likely dominated by the early time contribution. Similar results are shown in figure 4.5 .6 for the $0.5 \mu \mathrm{m}, 3.33$ ps case. Note, for 3.33 ps laser pulses with fluences above the substrate damage threshold, both the $0.5 \mu \mathrm{m}$ and $0.25 \mu \mathrm{m}$ Al films produce plumes with the approximately the same peak interface temperature in HYADES calculations. This indicates that, in this short time scale, heat diffusion outside the interface region is limited to much less than the thickness of the $0.25 \mu \mathrm{m}$ and $0.5 \mu \mathrm{m}$ films. 


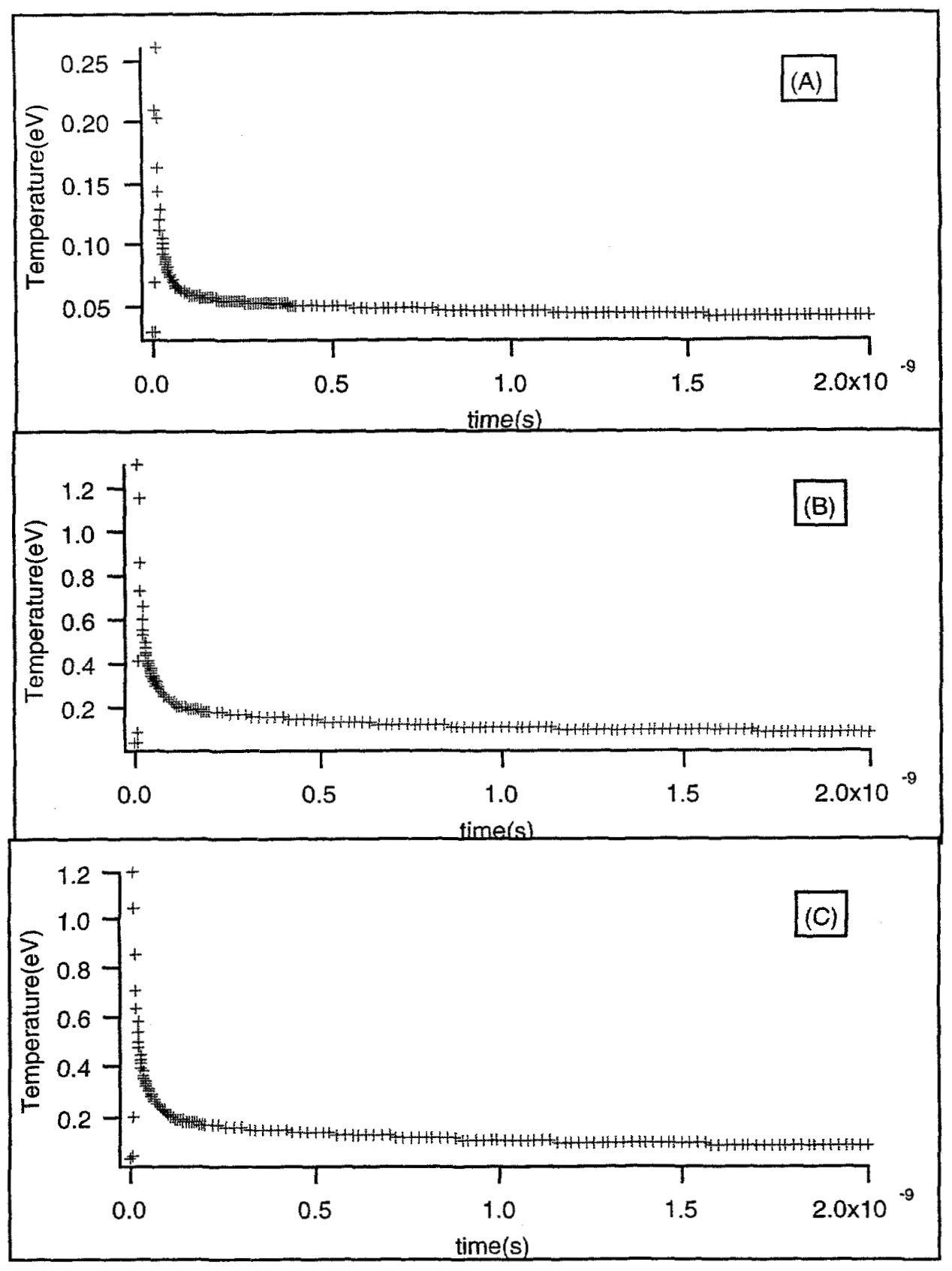

Figure 4.5.5. HYADES calculations of interface zone temperature as a function of time for plumes generated by $0.25 \mu \mathrm{m}$ films ablated by 3.33 ps pulses. Plot (A) corresponds to a laser pulse with $0.79 \mathrm{~J} / \mathrm{cm}^{2}$ peak front surface fluence. Plot (B) corresponds to a laser pulse with $7.18 \mathrm{~J} / \mathrm{cm}^{2}$ peak front surface fluence. Plot (C) corresponds to a laser pulse with $22.3 \mathrm{~J} / \mathrm{cm}^{2}$ peak front surface fluence. 


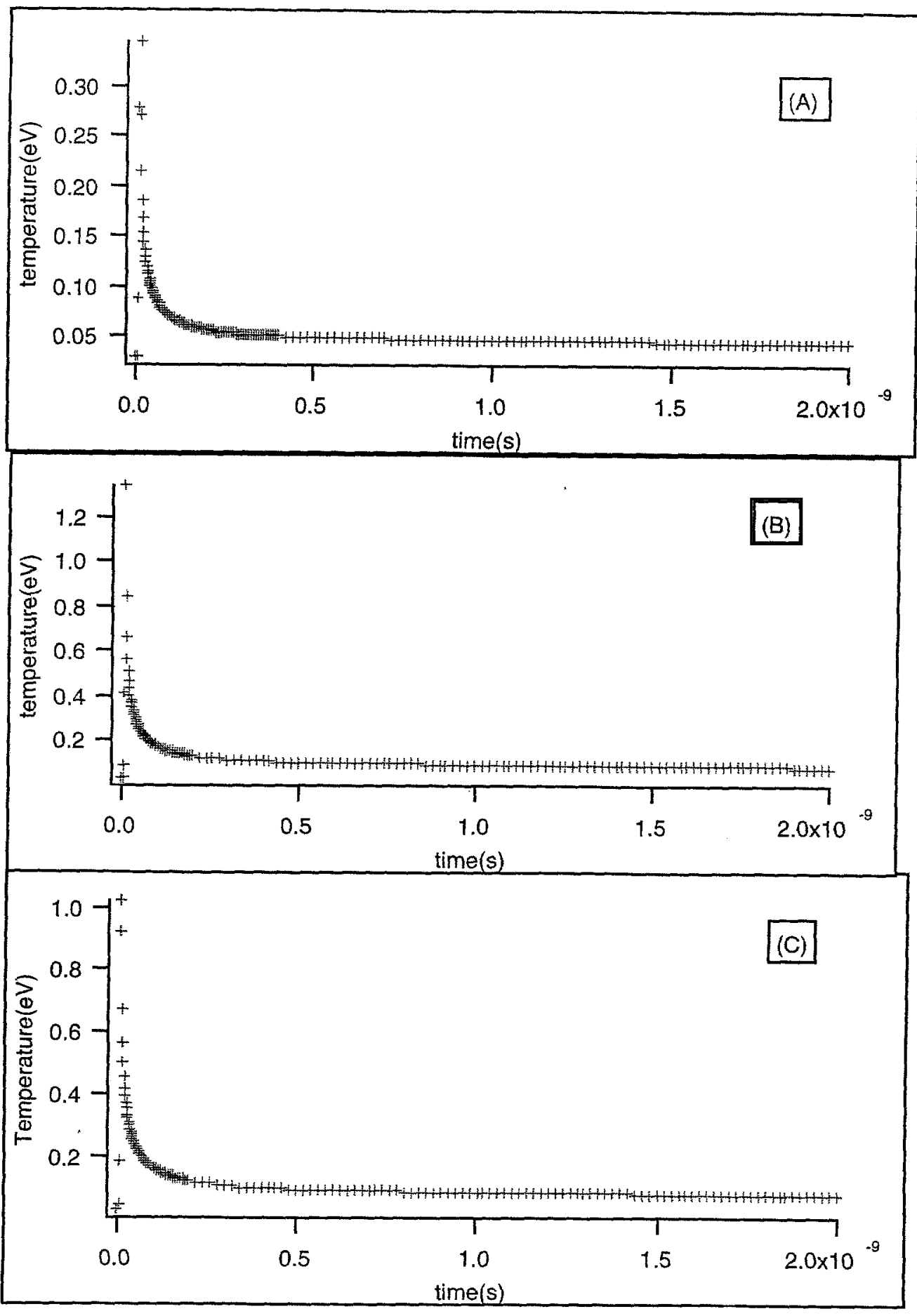

Figure 4.5.6. HYADES calculations of interface zone temperature as a function of time for plumes generated by $0.5 \mu \mathrm{m}$ films ablated by 3.33 ps pulses. Plot (A) corresponds to a laser pulse with $0.8 \mathrm{~J} / \mathrm{cm}^{2}$ peak front surface fluence. Plot (B) corresponds to a laser pulse with $5.38 \mathrm{~J} / \mathrm{cm}^{2}$ peak front surface fluence. Plot (C) corresponds to a laser pulse with 15.7 $\mathrm{J} / \mathrm{cm}^{2}$ peak front surface fluence. 
Also, the time scale for heat loss is much shorter than the time scale for expansion, which confirms that this drop in temperature is due to heat diffusion through the thickness of the film and not thermodynamic expansion of the interface zone. In figure 4.5.7, interface

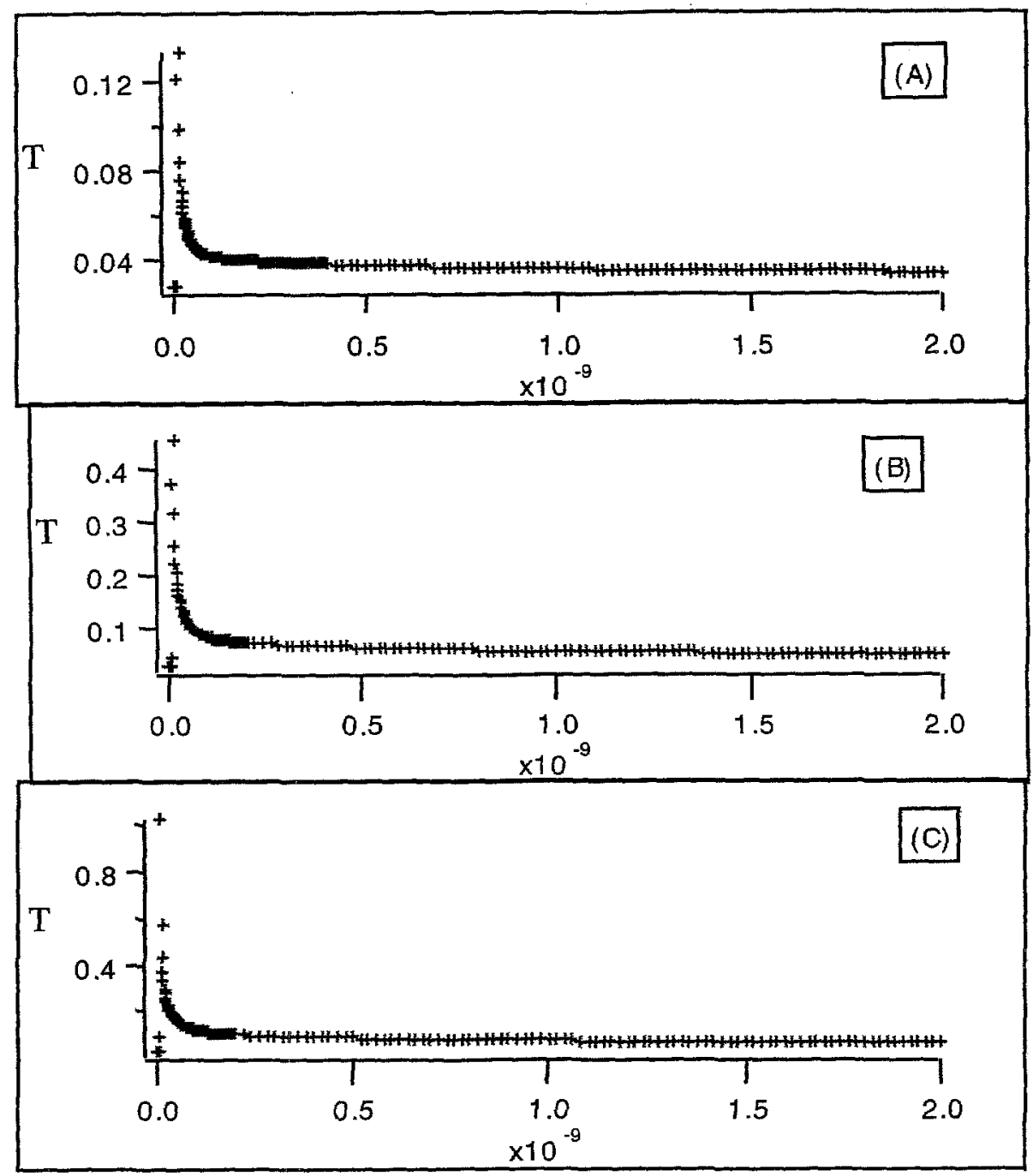

Figure 4.5.7. Plot of HYADES-calculated temperature, $T$, as a function of time for plumes generated by $0.25 \mu \mathrm{m} \mathrm{Al} \mathrm{films} \mathrm{ablated} \mathrm{by} 1.85$ ps pulses. Plot (A) corresponds to a laser pulse with $0.918 \mathrm{~J} / \mathrm{cm}^{2}$ peak front surface fluence. Plot (B) corresponds to a laser pulse with $3.6 \mathrm{~J} / \mathrm{cm}^{2}$ peak front surface fluence. Plot (C) corresponds to a laser pulse with $12.8 \mathrm{~J} / \mathrm{cm}^{2}$ peak front surface fluence.

temperature as a function of time for the $0.25 \mu \mathrm{m}, 1.85$ ps case is shown. Figure 4.5 .7 (C) shows the peak interface temperature is similar to that of the 3.33 ps case. Also, in figures 
4.5.5-4.5.7, the temperature drops to a low asymptotic value within 2 ns after the ablation pulse. These calculations show a low interface temperature at very late times consistent with the observed divergence discussed previously and also further confirm the existence of a hot plasma core inside the plume, near the interface, in early time.

\subsection{Discussion of Practical Aspects of Short-Pulse LIBA}

One of the more intriguing aspects of the LIBA process in the picosecond laser pulse regime is the substrate damage effect on plume behavior. Since the edge velocity saturation threshold fluence $\left(\mathrm{J}_{\mathrm{th}}=5.2 \mathrm{~J} / \mathrm{cm}^{2}\right.$ for the $0.25 \mu \mathrm{m}, 3.33$ ps case $)$ is within the range of reasonably available laser fluence values, one can shape ablated Al plumes by using the substrate damage mechanism to alter the transmitted spatial laser profile. Figure 4.6.1 shows the shadowgraphs of three Al plumes (each image captured at $255 \mathrm{~ns}$ of delay), each generated with 3.33 ps laser pulses of increasing fluence. The initially gaussian-like shape becomes blunted as fluence is increased. Since this effect is a function of incident intensity, however, one can use laser pulsewidth as a control to alter the shape of the Al plume. Figure 4.6.2 shows two plume shadowgraphs (each captured at $255 \mathrm{~ns}$ of delay), each of similar peak front surface fluence but each one of different pulsewidth. Note the sharp difference in plume profile. Plume (A) shows a blunted gaussian shape, while plume(B) has a sharply defined box-like shape. Since, for fluences under the substrate damage threshold, edge 


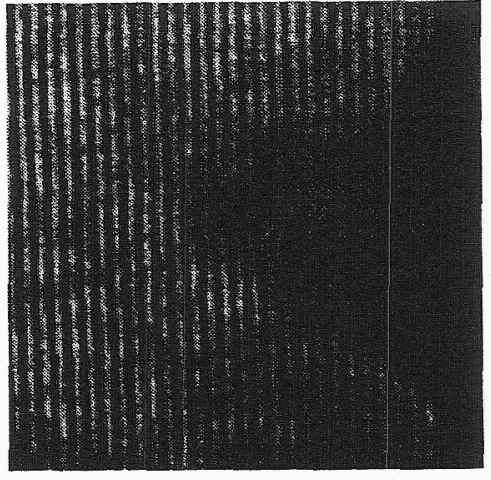

(A)

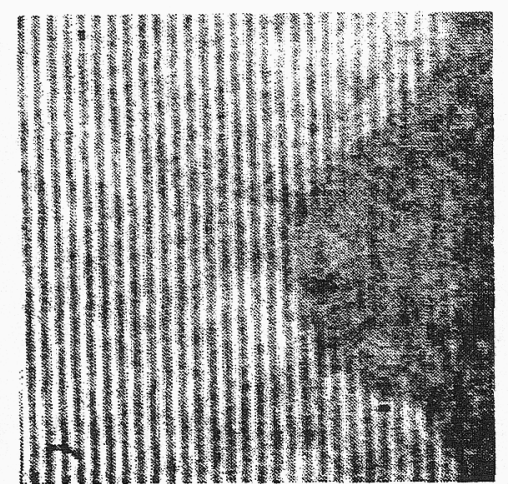

(B)

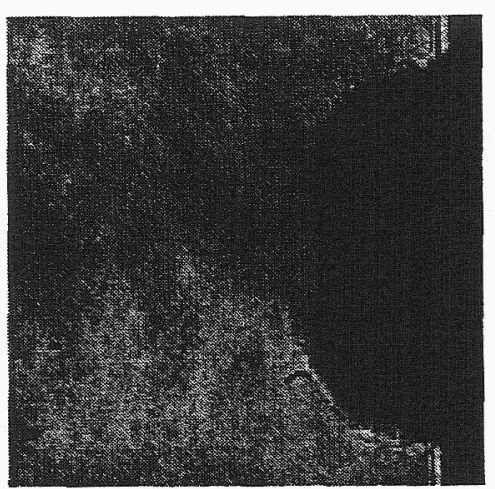

(C)

Figure 4.6.1. Interferograms of $0.5 \mu \mathrm{m} \mathrm{Al}$ film ablated with 3.33 ps pulses. [Peak front surface fluence $(A)=3.28 \mathrm{~J} / \mathrm{cm}^{2},(B)=4.68 \mathrm{~J} / \mathrm{cm}^{2}$, and $\left.(C)=12.1 \mathrm{~J} / \mathrm{cm}^{2}\right]$. These images were captured at $255 \mathrm{~ns}$ after arrival of ablation pulse.

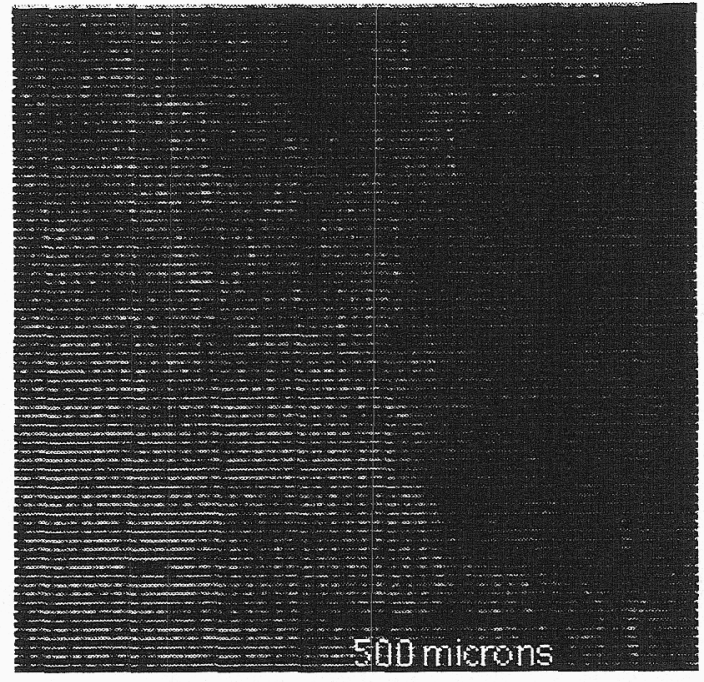

(A)

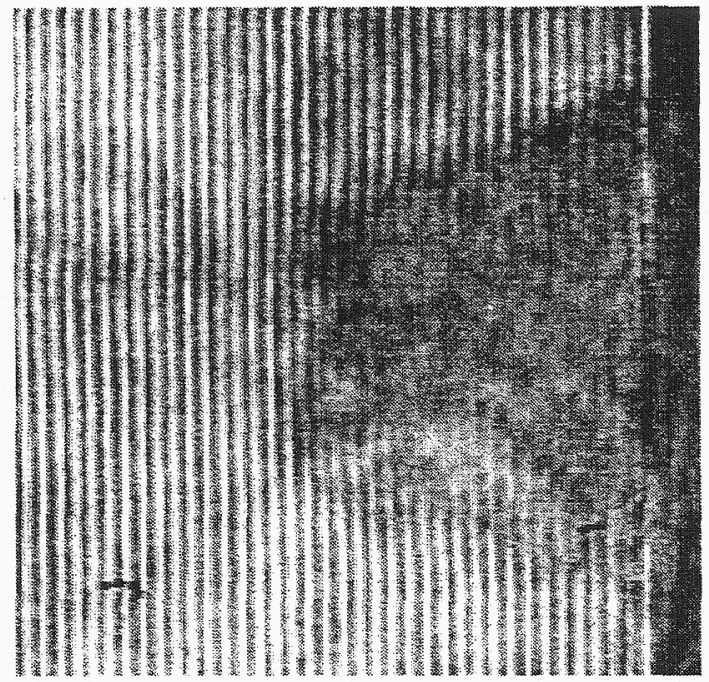

(B)

Figure 4.6.2. Interferograms $(527 \mathrm{~nm})$ of two Al plumes, generated with $0.25 \mu \mathrm{m}$ Al film ablated with either a 1.85 ps laser pulse (A) or a 3.33 ps laser pulse (B) (peak front surface fluence $(A)=21.5 \mathrm{~J} / \mathrm{cm}^{2},(B)=19.0 \mathrm{~J} / \mathrm{cm}^{2}$ ) These images were captured at $255 \mathrm{~ns}$ after arrival of ablation pulse.

velocity as a function of incident fluence has a square root functional form, one could selectively produce plumes with specified edge velocity and shape by changing both the incident fluence and incident pulse duration. The diffraction grating-based pulse compressor used in this experiment is well-suited for pulse duration control, and one 
could quite readily produce a specified plume by changing the compressor grating separation and changing the voltage setting on the sequential amplifiers. In applications such as using $\mathrm{Al}$ plumes as laser-plasma targets ${ }^{15}$, where target location is highly important in interpreting experimental results, this controllable plume edge can produce very favorable results where more established methods (such as longer pulse (10ns) LIBA, conventional front surface ablation) can be more problematic. Further, edge control is crucial to applications such as LIBA-generated ultrasound. In this application, the plume can act as a ultrasonic driver, and a flat plume profile allows meaningful use of this method to probe materials for bulk defects ${ }^{16}$. In material redeposition studies, laser pulses well within the substrate damage regime are almost "flat-top" profile at the metal/substrate interface, and this "flat-top" profile can greatly simplify understanding of plume divergence. Also, when coating flat receiving substrates, a flat plume profile would be highly advantageous in the production of thin films.

Since the substrate damage mechanism is a highly important control in short pulse LIBA, optical substrate damage emission from substrate damage can be an important LIBA monitor. In figure 4.6.3, the brightness and size of the substrate damage spot shown in the images is directly related to the ionization caused by the incident laser pulse. In this way, the oblique view CCD can be used to evaluate the transmitted fluence through the substrate. The size of the light emission spot in the substrate damage region of the substrate can also be used to predict the shape of the ablated plume. In figure 4.6.4(A), the substrate damage mechanism has already 


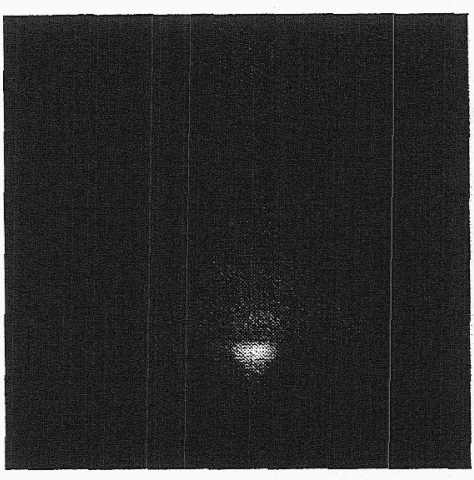

(A)

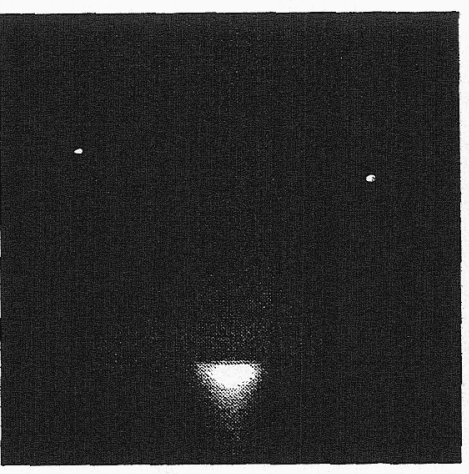

(B)

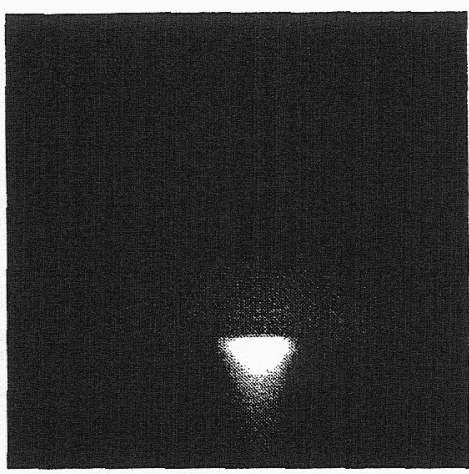

(C)

Figure 4.6.3. Oblique view CCD time-integrated images of in-flight plume and substrate damage emission for $0.25 \mu \mathrm{m}$ films ablated by 3.33 ps pulses. Image (A) has a peak front surface fluence of $22.6 \mathrm{~J} / \mathrm{cm}^{2}$, image (B) has a peak front surface fluence of $31.8 \mathrm{~J} / \mathrm{cm}^{2}$, and image (C) has a peak front surface fluence of $43.3 \mathrm{~J} / \mathrm{cm}^{2}$.

blunted the plume profile, although the outer edges shown the gaussian-like shape. These outer edges become less gaussian and more like a "flat-top" as the substrate damage spot increases in size(see figures 4.6.4(B) and 4.6.4(C)). This connection between substrate damage spot size and plume shape can be used during an experiment to monitor plume shape on a

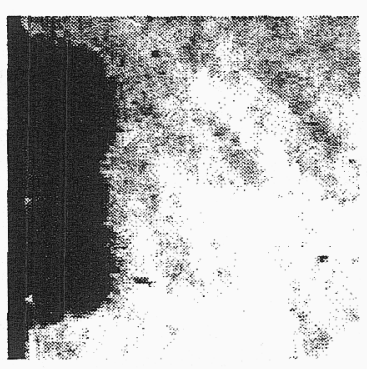

(A)

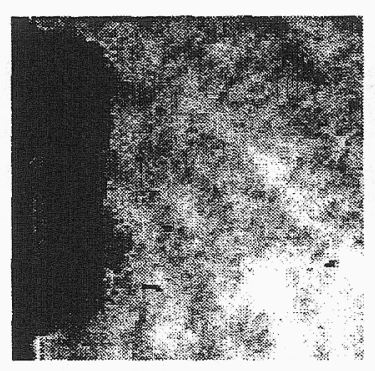

(B)

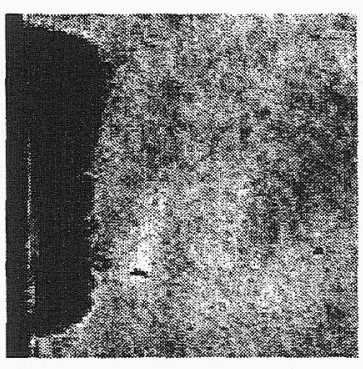

(C)

Figure 4.6.4. Shadowgraph images of in-flight plume generated by $0.25 \mu \mathrm{m}$ films ablated by 3.33 ps pulses. Image (A) has a peak front surface fluence of $22.6 \mathrm{~J} / \mathrm{cm}^{2}$, image (B) has a peak front surface fluence of $31.8 \mathrm{~J} / \mathrm{cm}^{2}$, and image (C) has a peak front surface fluence of $43.3 \mathrm{~J} / \mathrm{cm}^{2}$. 
shot-to-shot basis. An additional benefit of the oblique view CCD camera is the ability to monitor pulsewidth. Figure 4.6.5 shows oblique CCD images for LIBA in the $0.25 \mu \mathrm{m}$, $1.85 \mathrm{ps}$ case. Here, the substrate damage, and therefore the plume blunting effect, is much more pronounced for the same fluence range. This is reasonable, since substrate damage is related to intensity. This visible evidence of substrate damage allows the experimenter to roughly estimate the incident laser intensity. Another additional advantage is the longlived plume emission. The plume emission shown in figure 4.6.3 (A)-(C) allow the experimenter to estimate relative plume divergence and temperature.

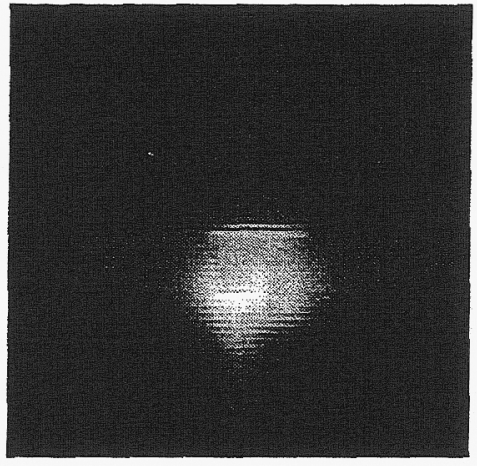

(A)

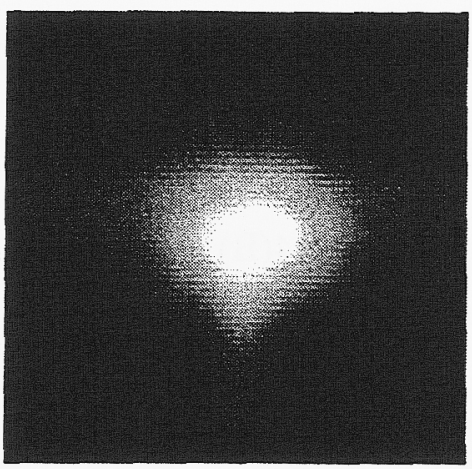

[B\}

Figure 4.6.5. Oblique view CCD time-integrated images of in-flight plume and substrate damage emission for $0.25 \mu \mathrm{m}$ films ablated by $1.85 \mathrm{ps}$ pulses. Image (A) has a peak front surface fluence of $20.1 \mathrm{~J} / \mathrm{cm}^{2}$ and image (B) has a peak front surface fluence of 29.3 $\mathrm{J} / \mathrm{cm}^{2}$.

The observed spectra can also be used as a LIBA monitor. Observed spectra can be used to monitor the substrate damage fluence limiting mechanism, measure the average Al plume temperature, and confirm the laser/target interaction geometry. Figure 4.6.6(A) and 

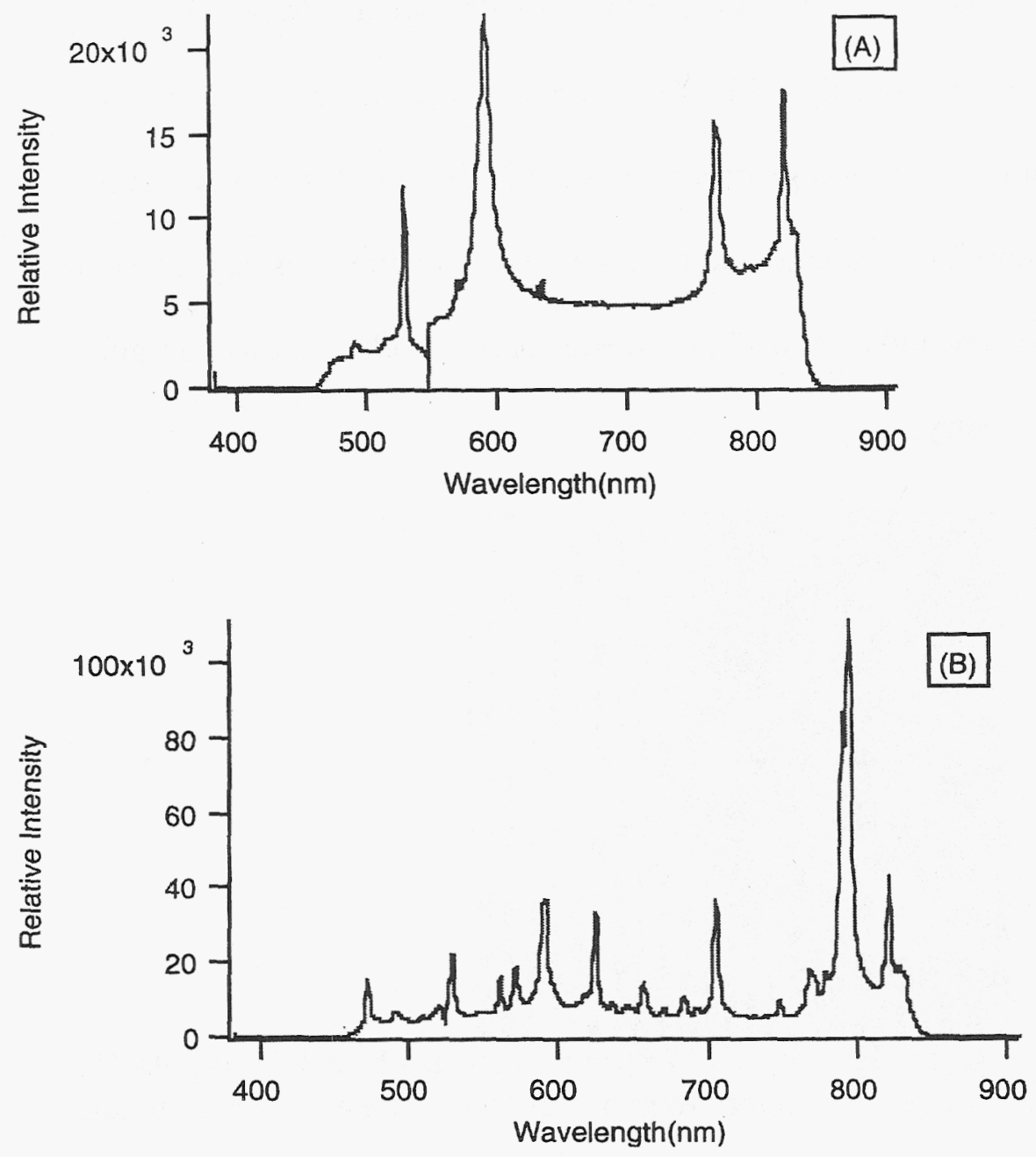

Figure 4.6.6. Plot of observed spectra for $0.25 \mu \mathrm{m}$ film ablated by $3.33 \mathrm{ps}$ pulse $\left((A)=11.1 \mathrm{~J} / \mathrm{cm}^{2}\right.$ peak front surface fluence and $(B)=12.3 \mathrm{~J} / \mathrm{cm}^{2}$ peak front surface fluence). Spectra (A) corresponds to the unclipped geometry, while spectra (B) corresponds to the clipped geometry.

(B) show the strikingly different spectra produced by changes in target geometry. During LIBA experiments, spectral emission such as shown in figure 4.6.6(B) would indicate the beam is clipping the target. A spectra such as shown in figure 4.6.6(A) would indicate a largely unclipped spectra, but it would also show substrate damage. In figure 4.6.6(A), the substrate damage is shown in the prominent lines at $590 \mathrm{~nm}$ and $820 \mathrm{~nm}$. When these 
lines, specifically the $590 \mathrm{~nm}$ line, are weak, the substrate damage mechanism is minimal, and one can expect a largely unblunted Al plume. When lines such as $590 \mathrm{~nm}$ and $655 \mathrm{~nm}$ are present in the clipped geometry, however, this indicates front surface substrate breakdown emission is leaking into the spectral collection optics, and substrate damage is occurring. This would lead to a blunted edge profile such as shown in figure 4.6.7. In the clipped geometry,

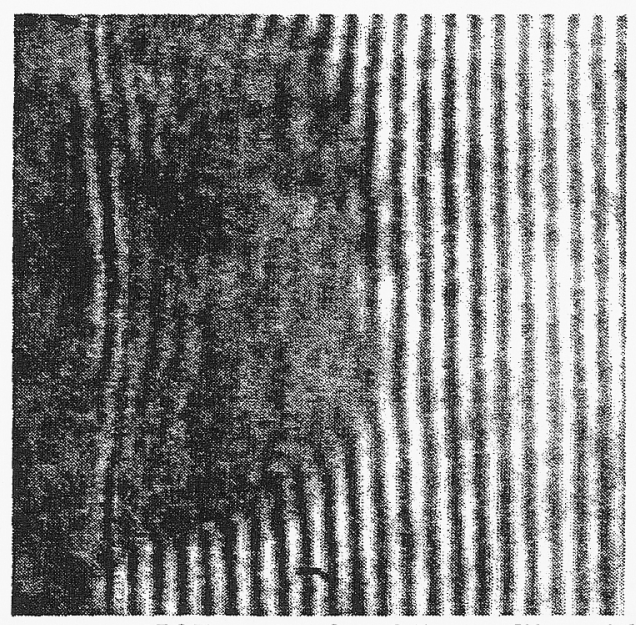

Figure 4.6.7. An interferogram $(527 \mathrm{~nm})$ of a $.25 \mu \mathrm{m}$ film ablated by a 3.33 ps pulse $(11.1$ $\mathrm{J} / \mathrm{cm}^{2}$ peak front surface fluence). The interferogram was taken with a $255 \mathrm{~ns}$ delay after the ablation event.

the relative ratio between the $470 \mathrm{~nm} \mathrm{AlIII}$ and the $559 \mathrm{~nm} \mathrm{Al} \mathrm{II} \mathrm{line} \mathrm{is} \mathrm{a} \mathrm{simple} \mathrm{monitor} \mathrm{of}$ Al plume temperature. Using the Saha equation, we find the ratio $\varepsilon_{470 \mathrm{~nm}} / \varepsilon_{559 \mathrm{~nm}} \approx 1$ equates to an early time temperature of $1 \mathrm{eV}$. Similarly, the ratio $\varepsilon_{470 \mathrm{~nm}} / \varepsilon_{624 \mathrm{~nm}} \approx 0.5$ equates to an early time temperature of $1 \mathrm{eV}$. Using these values as a benchmark, a visual inspection of this ratio can give a quick temperature estimate of the average Al temperature.

As a material deposition technique, LIBA has several advantageous features. LIBA removes the entire film thickness under ablation. Conventional front surface laser ablation with $10 \mathrm{~ns}$ pulses typically only removes $1-10 \mathrm{~nm} / \mathrm{pulse}^{17}$, a depth on order of the 
optical skin depth. Furthermore, the divergence of LIBA plumes is extremely low. On the edges, the divergence angle is measured to be less than 5 degrees. If we fit the edges of the plume to the standard functional form for divergence, $\cos ^{\mathrm{p}} \theta, \mathrm{p}$ values greater than 750 are required to fit the observed divergence. This is much more directed than conventional front surface ablation. For example, titanium ablated by $20 \mathrm{~ns}, 1064 \mathrm{~nm}$ pulses has a divergence functional form of $\cos ^{6.7} \theta^{18}$. As one can see, the product of low divergence and high material removal compared to conventional ablation would suggest LIBA would be highly efficient as a material deposition process. An additional advantage is the Al target hole size saturation seen at high front surface fluence. As front surface fluence increases beyond the substrate damage threshold, the hole size saturates. This feature allows the experimenter to make a LIBA-based deposition process relatively insensitive to changes in pulse fluence. For fluences well into the asymptotic regime, small changes in fluence or spatial pulse profile do not lead to changes in plume edge profile or velocity. This inherent stability in LIBA allows fine control of plume profile on a shot-toshot basis.

\footnotetext{
${ }^{1}$ C. Max, Theory of the Coronal Plasma in Laser Fusion Targets (Lawrence Livermore National Laboratory), p. 7.

${ }^{2}$ B.C.Stuart, M.D. Feit, S. Herman, A.M. Rubenchik, B.W. Shore, and M.D. Perry, Phys. Rev. B, 531749 (1996).

${ }^{3}$ R. Wangness, Electromagnetic Fields, p. 388.

${ }^{4}$ H.M. Milchberg and R.R. Freeman, J. Opt. Soc. Am. B 6, 1351 (1989).

${ }^{5}$ J. Larson, "Hyades User manual", p.8.

${ }^{6}$ LANL SESAME database (7/10/98).

${ }^{7}$ F.J. Mayer and G.E. Busch, J. Appl. Phys, 57, 827 (1985).

${ }^{8}$ J.H. Weaver, C. Krafka, D.W. Lynch, and E.E. Koch, Optical Properties of Metals, (Fachinformationszentrum Energie, Karlsruhe, 1981), p.75.

${ }^{9}$ J.F. Shackelford, Introduction to Material Science for Engineers, p. 624.

${ }^{10}$ M.S. Dimitrijevic and S. Sahal-Brechot, Astron. Astrophys. Suppl. Ser. 99, 585 (1993).

${ }^{11}$ H.R. Griem, Plasma Spectroscopy., McGraw-Hill, New York (1964).

${ }^{12}$ G. Bekefi, Principles of Laser Plasmas, John Wiley and Sons, New York (1976).

${ }^{13}$ Y.B. Zeldovish and Y.P. Raizer, Physics of Shock Waves and High Temperature Hydrodynamic Phenomena, pp..217-218.

${ }^{14}$ R. W. McWhirter, "Spectral Intensities," in Plasma Diagnostic Techniques, R. H. Huddlestone and S.L. Leonard (Eds.), Academic, New York (1965).
} 
${ }^{15}$ P.R. Bolton, D.C. Eder, G. Guethlein, R.E. Stewart, and P.E. Young, in Short-Pulse High-Intensity Lasers and Applications II, H.A. Baldis, ed., Proc. Soc. Photo-Opt. Instrum. Eng. 1890, 167 (1993).

${ }^{16} \mathrm{~T}$. Hale, LANL, private communications.

${ }^{17}$ R. Kelly and A. Miotello, "Mechnaisms of Pulsed Laser Sputtering", inPulse Laser Deposition of Thin Films, D.B. Chrisey and G. K. Hubler, eds., p. 64 (1994).

${ }^{18}$ Ibid., p.205. 


\section{CHAPTER 5: CONCLUSIONS}

\subsection{Introduction}

This dissertation has been an exploratory study of the novel ablation process, Laser-Induced Back-Ablation (LIBA), in the picosecond laser pulse regime. This study has used several diagnostic tools, such as time-delayed shadowgraphy/interferometry, CCD-based video imaging, time-integrated spectroscopy, and target post-processing analysis, to understand LIBA as both a physical process and as an engineering technique. LIBA has been found to have many useful characteristics in this pulse regime, including high directivity, high density, convenient geometry, and controllable edge velocity and plume profiles. The study also detailed the practical aspects of applying LIBA technology in the laboratory, including the utility of substrate damage as a process control. Together, this body of research suggests interesting new areas of LIBA research.

\subsection{LIBA Diagnostics}

Each LIBA diagnostic tool revealed important aspects of the LIBA process. Timeintegrated spectra showed evidence of substrate damage in both uncoated BK-7 substrates and coated Al targets, as well producing temperature estimates of the hot core region of the $\mathrm{Al}$ film in early time. The $\mathrm{CCD}$-based images of the plume emission revealed evidence of front substrate ablation and $\mathrm{Al}$ film ablation. The time-delayed shadowgraphs showed plume edge profiles and edge velocities. Two-color interferometry showed the neutral atom and free electron density in the plumes. The post-processing analysis produced estimates of the $\mathrm{Al}$ film ablation threshold as well as $\mathrm{Al}$ deposit divergence. 
Collected optical spectra of uncoated BK-7 substrates under laser-induced ablation show a spectra composed of Si I, Si II, and Si III lines. Strong lines at $590 \mathrm{~nm}$ and $820 \mathrm{~nm}$ are seen in these spectra, and the clear observation of these lines indicate that substrate ionization and damage is occurring. Also, the distinctive shape of the spectra originating from the uncoated substrates is very much unlike a blackbody spectra, and this shape can also be used to identify laser-induced substrate ionization. Optical spectra collected from the Al target in the "clipped" geometry show the presence of a hot region (temperatures of order $1 \mathrm{eV}$ ) inside the $\mathrm{Al}$ film near the interface in early time $(<100 \mathrm{ps})$. This temperature is an approximately constant function of front surface fluence, indicating that front-surface substrate damage is regulating the core plume temperature. In the unclipped geometry, a blackbody spectra is observed for low incident fluence, suggesting that the total Al temperature is far lower than $1 \mathrm{eV}$. Also, for high incident fluences, strong Si lines are also present in these unclipped and clipped spectra, further indicating substrate damage.

CCD time-integrated images show the optical emissions associated with laserinduced ionization at the front substrate surface at high laser fluences. These images can reveal the back-ablation event. The images of the back-ablated plume provided a rough measure of plume directivity, and examining the laser damage on the front surface led the experimenter to consider the relation between laser-induced substrate damage and plume velocity.

Interferograms and shadowgraphs show the plume to have a well-defined outer edge at long delay times of order 200-300 ns. Comparison of similar Al plumes at multiple time delay indicates very low divergence, and low observed divergence at these 
long times indicates very low temperatures. Two-color interferograms of $0.1 \mu \mathrm{m} \mathrm{Al} \mathrm{film}$ confirm a low ionization level (of order $0.1 \%$ ) at a delay of $255 \mathrm{~ns}$. Interferograms of thick films $(0.25 \mu \mathrm{m})$ and/or low fluence also show a region of higher temperature near the substrate and a colder region away from the substrate. This observation is a confirmation of the hot core suggested by the spectra, since, as the low-fluence plume expands, the hot region would be separated from the colder outer region by ablation prior to complete heat diffusion and temperature equilibrium. Edge velocity at higher fluence show a edge velocity saturation effect, which modeling shows be caused by fluencelimiting laser-induced substrate damage at the substrate front surface. This results is plumes with controllable edge profiles. The consequence of a cold, well-defined plume at long delay times is that, by controlling the substrate damage mechanism and the transmitted fluence profile, one can control plume shape at long delay times.

The post-processing analysis of the $\mathrm{Al}$ targets and recollected $\mathrm{Al}$ plumes after the experiment revealed interesting features. The Al target ablation hole size, when compared to the incident laser beam profile, showed the threshold fluence for ablation. The recollected $\mathrm{Al}$ plumes showed the divergence of the plume at the recollection point, further confirming the low plume divergence evidenced in both the shadowgraphs and the CCD images for late times.

\subsection{Useful aspects of Picoseond Pulse LIBA}

This study has shown picoseond-pulse LIBA to have many useful characteristics. Al plumes produced by LIBA have a high directivity and low divergence. Highly directed plumes are very suitable for material deposition, such as selective bonding, since low material divergence would be more economical and less wasteful. High density/low 
temperature plumes are highly useful in areas such as material deposition, laser/matter interaction studies, ultrasonic generation research, and ion injection research, since high density improves the efficiency of LIBA in each of these areas. The back-ablation geometry also allows the probing of the ejected plume material without undesirable interaction with the ablation pulse, further improving the ability to diagnosis and control LIBA plumes.

\subsection{LIBA as a Laboratory Process}

In the process of this experimental study, several practical aspects of short-pulse LIBA become apparent. The use of high incident laser fluence in LIBA causes the plumes to become relatively insensitive to small changes in laser fluence. Hot spots in the beam have a small effect of plume edge profile at higher incident fluences, and the plume takes on a highly-reproducable "flat" plume shape. Additionally, this study shows that extension of this research to pulsewidths below 1 ps is not promising for substrates with damage thresholds comparable to or less than that of BK-7. The substrate damage mechanism would prevent sufficient laser fluence to pass into the Al film to cause backablation. Also, although material deposition using LIBA would involve the ablation pulse striking the target in the unclipped geometry, the spectra shows the utility of clipping the target to reveal the early-time temperature of the hot Al film core. For this reason, splitting an ablation pulse into two equal portions and striking one target to produce the recollection while clipping another, identical target to produce clipped-geometry spectra would a useful technique to simultaneously probe the LIBA process while producing useful redepositions. The choice of substrate is also highly important. The roughness and material of a target substrate strongly affects the laser-induced damage mechanism, and 
by selecting the appropriate substrate, one has another method for controlling the laserinduced damage mechanism. Finally, use of LIBA as a practical research tool requires a minimum diagnostic setup of spectrometer, CCD-based imaging system, and shadowgraph system, since these three diagnostics, used in concert, reveal the important plume characteristics such as divergence, temperature, density, and velocity on a shot-toshot basis.

\subsection{Future Directions for LIBA Research}

Short and ultrashort pulse LIBA is a research opportunity highly suitable for additional exploration. The use of different substrate and target materials would be both interesting and potentially very useful in the material fabrication fields. Additionally, the potential of multiple-layer targets, where one layer acts as the ablation layer and explodes outward while the other layer, farther away from the substrate/film interface, acts as the projectile layer and is pushed away from the substrate by the ablation layer, is very interesting. In this case, the relevant material mixing in the plume can be investigated spectroscopically. A clever choice of multilayers might include a weakly adhering interfacial layer with another layer placed on top, thereby allowing ablation at lower fluences. Applying time-resolved spectroscopy would also be highly exciting and would allow probing the interaction in very early time. Also promising is the use of thirdharmonic UV shadowgraph probe pulses. The shorter wavelength would allow a deeper probing of the material and more sensitive neutral and electron density detection. Longer delay (on order microseconds) shadowgraphs would also be useful, since they allow probing of the plume just prior to redeposition. All these improvements make the further 
investigation of picosecond pulse LIBA highly intriguing and well worth additional effort. 


\section{APPENDIX A: A TI:SAPPHIRE-BASED CPA PICOSECOND LASER SYSTEM}

\section{A.1 Introduction}

The dynamics of short-pulse LIBA are determined in part by the characteristics

of the laser used for ablation. Pulse length, focal spot quality, temporal beam profile, and deliverable laser fluence all strongly affect the subsequent plume generation by LIBA. Therefore, an in-depth study of LIBA requires a good understanding of the ablation laser system. This section discusses the theory and operation of the Picosecond laser system at LLNL, as well as motivations for using short-pulse laser systems in LIBA.

\section{Basic Layout}

The Picosecond Laser system at LLNL is a hybrid Ti:Sapphire-based Chirped Pulse Amplification system. A Spectra-Physics 2040E argon-ion laser pumps a SpectraPhysics Tsunami oscillator tuned to $1053 \mathrm{~nm}$ output. The oscillator produces $1 \mathrm{~nJ}, 100$ fsec pulses at a rate of $82 \mathrm{MHz}$. Each pulse passes through a diffraction grating stretcher system where the pulse is linearly chirped to a duration of 1 nsec. This pulse is then used to seed a Ti:Sapphire-based Nd:YAG regenerative amplifier. The output of this amplifier is $4 \mathrm{~mJ}, 800$ psec pulses at a rate of $10 \mathrm{~Hz}$ ( gain $\mathrm{G} \approx 4 \times 10^{6}$ ). Each output pulse then propagates down the amplifier chain, where a serrated aperture and a series of vacuum spatial filters produce a "top-hat" spatial profile. Three single-pass amplifiers isolate one pulse in the $10 \mathrm{~Hz}$ pulse train for amplification, while two pulse slicers increase the amplified pulse/satellite pulse contrast ratio to $10^{9}$. Finally, the amplified pulse passes into a vacuum diffraction grating compressor system, where it is compressed down to a pulse duration ranging from 500 fsec to 3.33 psec. The pulse duration can be specified in this range. 


\section{A.2 Master Oscillator System}

Inside the Tsunami laser cavity (see Figure A.2.1), the argon-ion pump laser is focussed into a Ti:Sapphire laser rod. Four adjustable prisms are placed in the beam line, to provide GVD compensation and, with a slit, laser wavelength selection. An A/O modulator operating at $82 \mathrm{MHz}$ initiates mode locking and intensity-dependent Kerrlensing maintains stable pulsing. The output of the Tsunami oscillator is $100 \mathrm{fsec}, 1053$ $\mathrm{nm}$ pulses at a rate of $82 \mathrm{MHz}$ with an average power of $140 \mathrm{~mW}$. These pulses are propagated through a 4-pass diffraction grating pulse stretcher and are stretched into 1 nsec, linearly chirped pulses suitable for seeding the regenerative amplifier.

A Ti:Sapphire rod provides a gain medium for the Tsunami. Ti:Sapphire has an energy band structure that is very advantageous for ultra-short pulse lasers. Strong coupling between $\mathrm{Ti}^{3+}$ and the $\mathrm{Al}_{2} \mathrm{O}_{3}$ lattice allows for broad fluorescence and absorption bands (see figure A.2.2) ${ }^{1}$. This strong coupling also allows for large energy separation between the absorption and fluorescence spectra (see figure A.2.3). A large energy separation between bands is important for efficient laser operation ${ }^{2}$. Also, a broad fluorescence band is essential for short pulse generation, since very short mode-locked laser pulses have broad spectral bandwidth which is composed of a large number of axial modes, and the gain medium must amplify all of these modes to produce such a pulse ${ }^{3}$. 


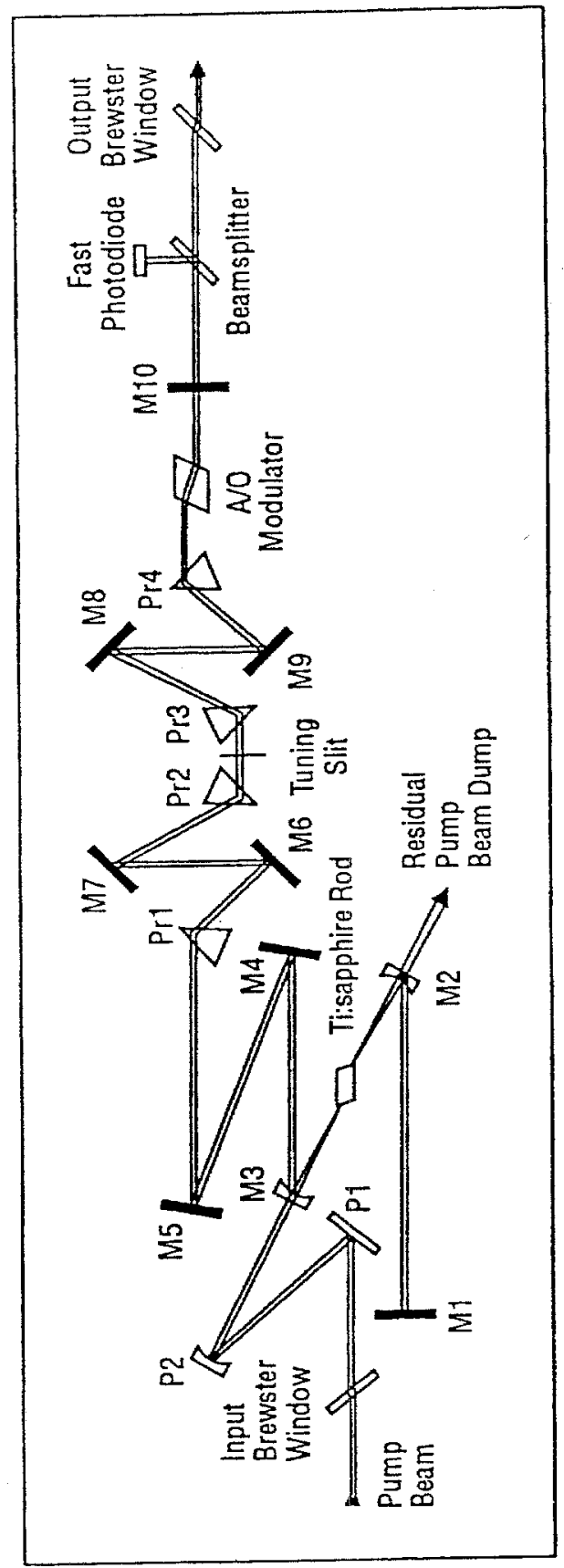

Figure A.2.1. Diagram of a Spectra-Physics Ti:Sapphire-based Tsunami Oscillator ${ }^{4}$

This gain medium is pumped by a continuous wave (cw) $514.5 \mathrm{~nm}$ argon-ion laser with a power of approximately $9.5 \mathrm{~W}$. The pump has a $\mathrm{TEM}_{00}$ spatial mode 


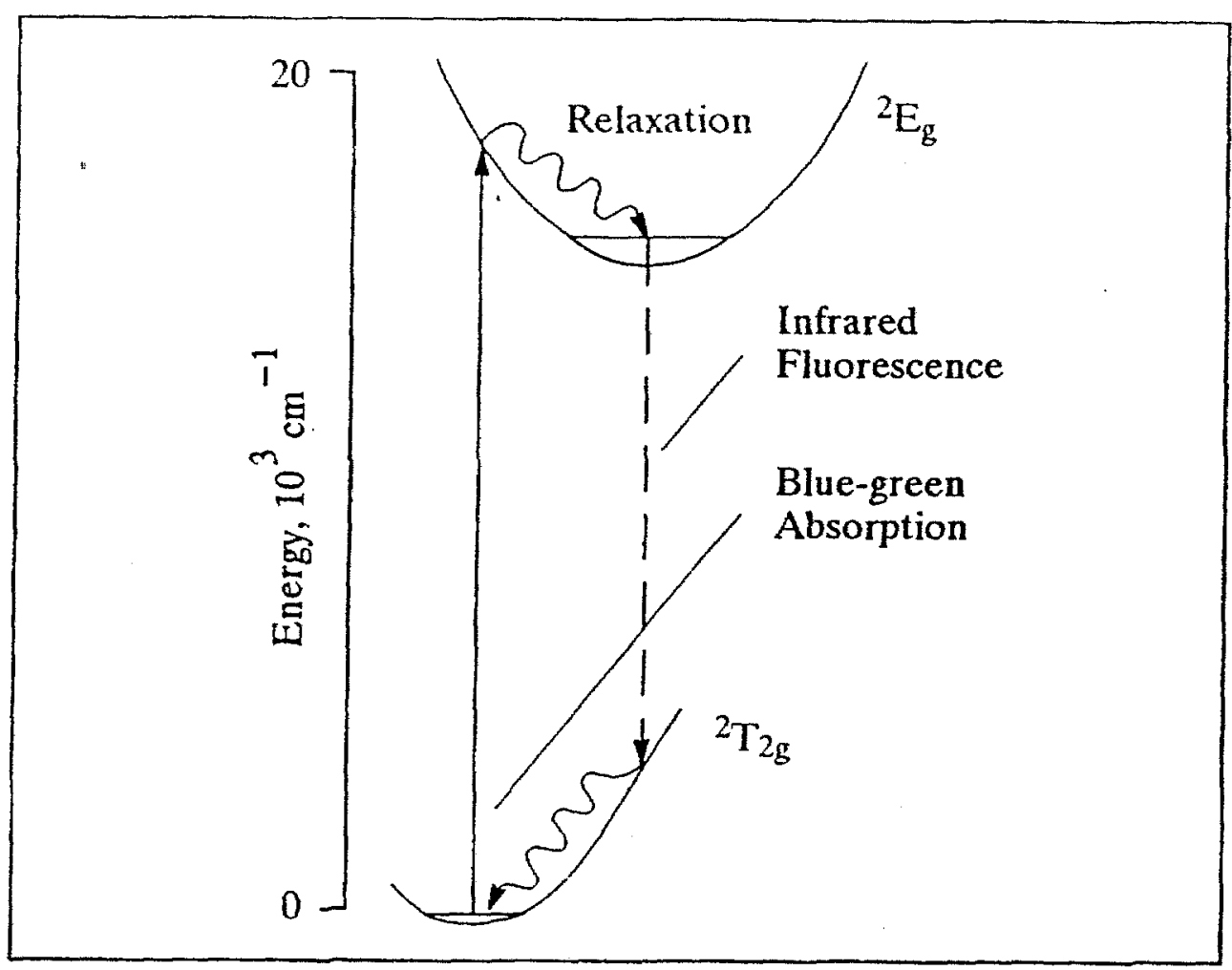

Figure A.2.2. Energy Band Structure for Ti:Sapphire ${ }^{5}$.

beam profile ${ }^{6}$ and this mode sets up a TEM To $_{0}$ mode in the Tsunami cavity. With this pumping, the laser can operate in a CW mode at frequencies within the emission spectrum.

Although it can lase in an unlocked cw mode, the Tsunami was designed to generate ultra-short pulses of approximately $100 \mathrm{fs}$ in a user-specified wavelength. The Tsunami oscillator is designed with both active and passive mode-locking. Mode-locking is a process where a large number of axial cavity modes are phase-locked with respect to each other. Adding the electric field components for a large number of modes, $\mathrm{N}$, locked together in phase, the total field and intensity can be described with the expression below ${ }^{7}$. 


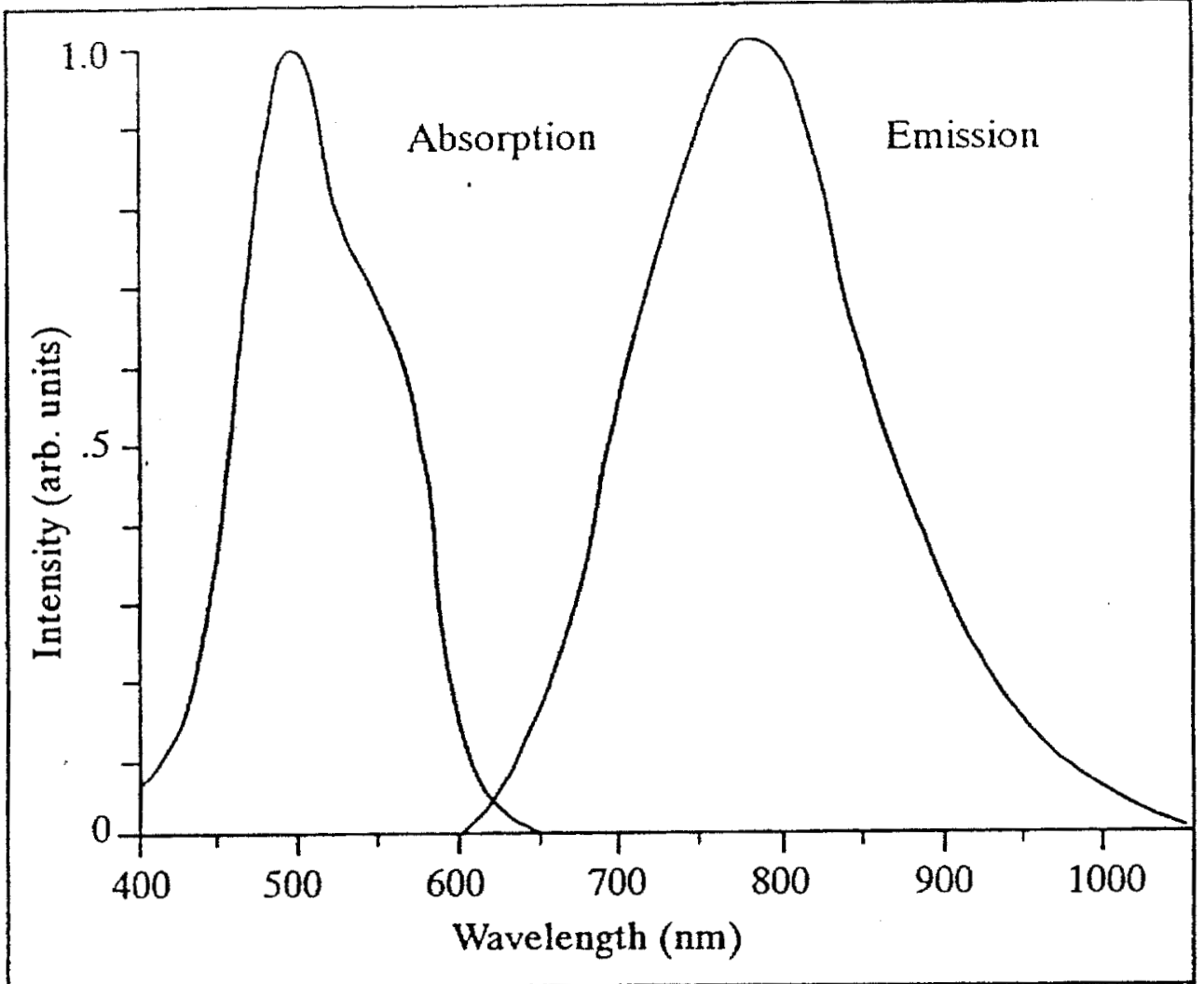

Figure A.2.3. Absorption and Emission Spectra for Ti:Sapphire ${ }^{8}$

$$
\begin{aligned}
& E(\omega)=E_{0} \sum_{n=0}^{N-1} \delta\left(\omega-\omega_{0}-n \omega_{a x}\right) \\
& E(t)=\int_{-\infty}^{\infty} E(\omega) e^{i \omega t} d \omega=E_{0} \frac{e^{i N \omega_{\alpha x}}-1}{e^{i \omega_{a r^{r}}}-1} e^{i \omega_{0} t}
\end{aligned}
$$

$I(t)=|\mathrm{E}(t)|^{2}=\left|\mathrm{E}_{0}\right|^{2} \frac{1-\cos N \omega_{a x} t}{1-\cos \omega_{a x} t}=\left|\mathrm{E}_{0}\right|^{2} \frac{\sin ^{2} N \omega_{a x} t / 2}{\sin ^{2} \omega_{a x} t / 2}$, where $\omega_{a x}$ is the axial mode spacing frequency $\omega_{\mathrm{ax}}=\omega_{\mathrm{q}+1}-\omega_{\mathrm{q}}=\pi \mathrm{c} / \mathrm{L}, \omega_{0}$ is the central frequency, and $\mathrm{L}$ is the laser cavity length. 
This expression, when plotted against time, represents a train of ultra-short laser pulses. For the Tsunami operating at $1053 \mathrm{~nm}$, mode-locking produces pulses of $\tau_{\mathrm{p}}<130 \mathrm{fsec}$. It produces this by using both an active mode-locking acousto-optic modulator and a passive Kerr-lensing passive mode locking scheme. The Tsunami includes an A/O modulator which oscillates between maximum and minimum transmission at a rate of 82 $\mathrm{MHz}$. In the frequency domain, this modulator produces modulation side-bands on an initial mode which corresponds to the frequency of another axial mode ${ }^{9}$ These sidebands then can injection-lock the resonance modes and lock the phases of the initial pulse and the resonance pulse together ${ }^{10}$. After initial mode locking, the subsequent increase in instantaneous pulse intensity produces Kerr-lensing inside the Ti:Sapphire rod ${ }^{11}$. The Kerr-lensing causes the lowest-level steady-state spatial mode to have a better overlap with the pump beam and lower losses, thereby allowing the mode-locked pulse to dominate the unlocked $\mathrm{CW}$ beam. This passive mode-locking reinforces the effect of the A/O modulator, and leads to a stable output of $130 \mathrm{fsec}$ pulses at $82 \mathrm{MHz}$.

The Tsunami also includes a set of four prisms in the beam line (see Figure A.2.4). These prisms serve two functions. The first function is to compensate for group velocity dispersion (GVD) ${ }^{12}$. As stated above, an ultra short pulse is composed of a large number of axial modes, and each mode has a fixed temporal relationship with all the other modes. As the pulse propagates through the laser cavity, positive GVD can cause the longer wavelength components of the pulse to advance in time relative to the shorterwavelength components. Excessive positive GVD can prevent a steady-state pulsing mode from forming, thereby preventing ultra-short pulse generation. Each pair of prisms in the beam line compensate for this positive GVD by producing a negative GVD. This is 
accomplished by aligning each prism in the pair such that the exit plane of the first prism and the entry plane of the second prism are parallel, and the beam enters the pair at the apex of the first prism. This causes the shorter-wavelength components of the pulse to advance in line relative to the long-wavelength components, thus producing a negative GVD which compensates for positive GVD in other parts of the laser cavity. The second function of the 4-prison system is to allow for wavelength selection. In the M-M' plane below, wavelength is mapped out along the plane. By placing a finite aperture slit in that plane place, only those wavelengths that will propagate through the slit will be supported in the cavity.

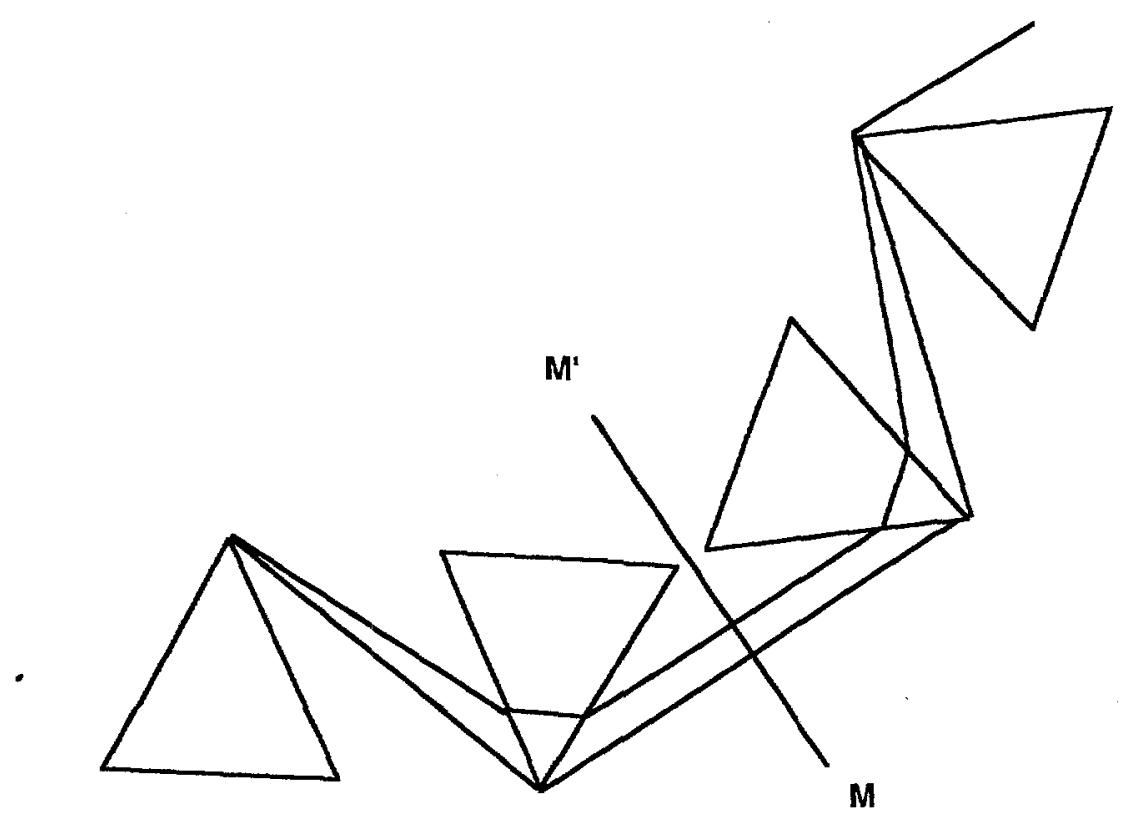

Figure A.2.4. Diagram of 4-prism GVD compensator

\section{A.3. Chirped Pulse Amplification}

Picosecond laser pulses are highly desirable for LIBA experiments. However, the generation of picosecond high-energy laser pulses suitable for LIBA presents non-trivial technical challenges. The direct generation and amplification of picosecond pulses at the $10 \mathrm{TW}$ level necessarily involves intensity-related issues in the laser system such as non- 
linear phase shifts, self-focusing, and laser-induced optical damage in optical components. These issues put a restrictive limit on the maximum intensities available from tabletop laser systems. A technique used to mitigate this problem is chirped-pulse amplification (CPA). CPA involves taking a low energy (nJ), ultra-short (140 fsec), large bandwidth $(14 \mathrm{~nm})$ laser pulse and propagating it into a diffraction-grating-based pulse stretcher. This pulse stretcher adds a controlled amount of group-velocity dispersion (GVD) into the pulse, transforming the input pulse into a $1 \mathrm{~ns}$, linearly-chirped pulse. This lowers the pulse intensity by a factor of 7000 prior to pulse amplification. Both the regenerative amplifier and the sequential amplifier chain now can safely amplify this pulse. After amplification, the pulse passes into a diffraction-grating-based pulse compressor, and additional GVD is added to the pulse to compensate for that added by the stretcher and the amplifier elements. This results, at maximum compression, in an output pulse of sub-picosecond (500 fs) duration and high energy (4J). One can also control the output pulse duration by deliberately altering the pulse compressor such that the GVD added to the pulse by the stretcher does not completely compensate for GVD introduced by the stretcher and amplifier elements. The output pulse then is a linearlychirped pulse of variable duration ( $500 \mathrm{fsec}-3.33 \mathrm{psec}$ in this experiment).

After the seed pulse is generated by the Tsunami oscillator, it propagates into the pulse stretcher (see figure A.3.1). 


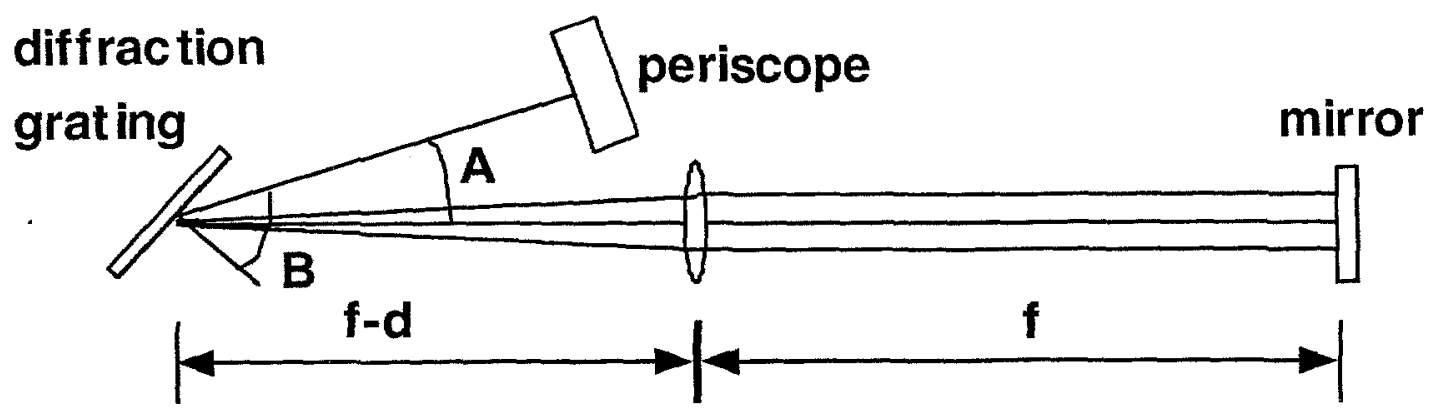

Figure A.3.1. Diagram of pulse stretcher system.

The pulse stretcher uses a diffraction grating to produce a frequency-dependent phase shift in the input pulse. If one expands the induced phase shift, $\exp (i \varphi(\omega))$, in frequencyspace, the phase can be described by the following expression:

$$
\varphi(\omega)=\varphi_{0}+\left.\frac{d \varphi}{d \omega}\right|_{0}\left(\omega-\omega_{0}\right)+\left.\frac{1}{2} \frac{d^{2} \varphi}{d \omega^{2}}\right|_{0}\left(\omega-\omega_{0}\right)^{2}+\ldots
$$

The pulse stretcher induces a quadratic phase shift in the laser pulse and the value of induced quadratic phase coefficient is shown below.

$$
\frac{d^{2} \varphi}{d \omega^{2}}=2 d \frac{\omega}{c}\left(\frac{\lambda}{2 \pi \sigma \cos \theta_{0}}\right)^{2}
$$

where $\sigma$ is the gaussian beam waist.

After passing through the pulse stretcher and receiving a quadratic phase shift, the laser pulse becomes linearly chirped and its pulse duration increases. If the input pulse has a gaussian temporal pulse shape described by the parameter, $\Gamma_{0}=\mathrm{a}-\mathrm{ib}$, and does not have any linear chirp $(b=0)$, the output pulse can be described by the modified parameter shown below. 


$$
\Gamma=\frac{a}{1+4 \frac{d^{2} \varphi}{d \omega^{2}} a}+i \frac{2 a^{2} \frac{d^{2} \varphi}{d \omega^{2}}}{1+4 \frac{d^{2} \varphi}{d \omega^{2}} a}
$$

This new parameter shows both the linear chirp and broadened pulsewidth induced by the pulse stretcher.

As the pulse leaves the stretcher, it then passes through the regenerative amplifier and the sequential amplifier chain and into the vacuum pulse compressor. The pulse compressor is a set of parallel diffraction gratings set a set distance apart (see figure A.3.2), and passage through the pulse compressor induces additional quadratic phase on to the pulse.

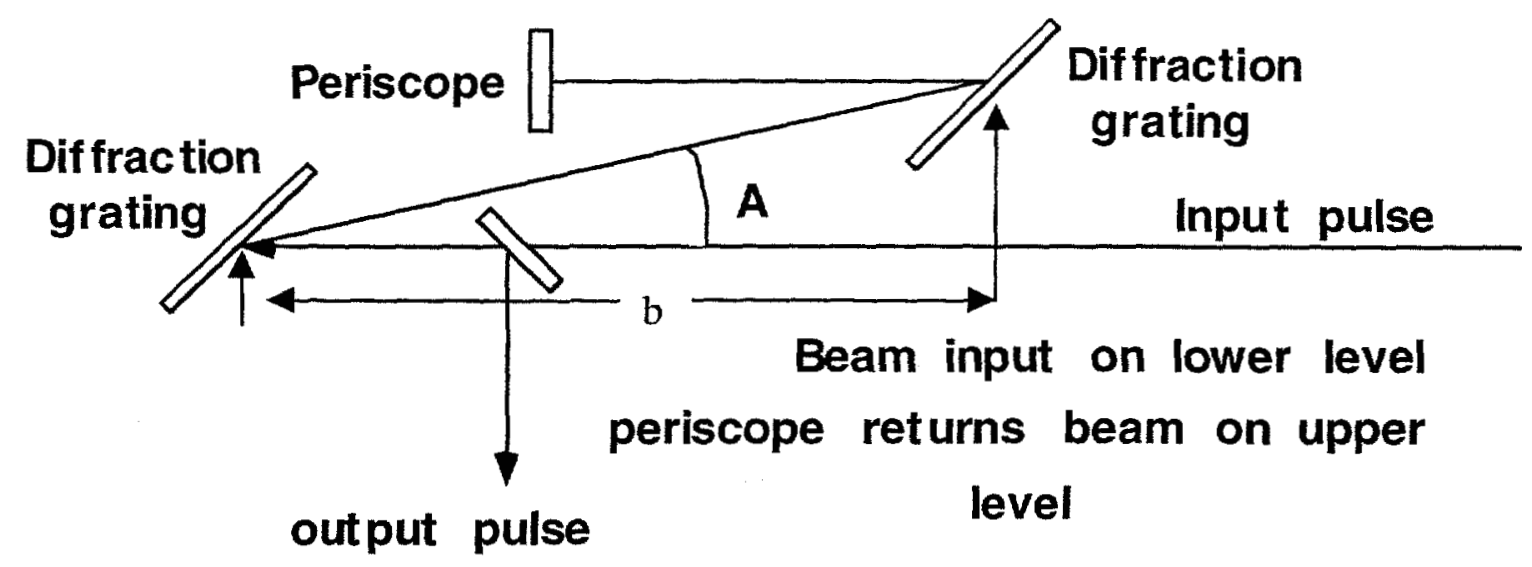

Figure A.3.2. Diagram of vacuum compressor system.

This quadratic phase is described by the following expression:

$$
\frac{d^{2} \varphi}{d \omega^{2}}=-\frac{\lambda}{2 \pi c^{2}}\left(\frac{\lambda}{d}\right)^{2} \frac{b}{\sqrt{r}} \frac{1}{r}
$$

where $r=1-[2 \pi c /(\omega d)-\sin (A)]^{2}, d$ is the grating constant (1/groove density), and $b$ is the normal separation of two gratings.

As can be seen in (A.4) and (A.5), a grating separation distance, b, can be chosen such that the GVD induced in the pulse by the pulse stretcher can be exactly compensated by 
GVD induced by the pulse compressor. In practice, this b value is selected by examining the intensity autocorrelations of the output pulse as a function of compression grating separation. At the proper value, the autocorrelation width is minimized, and the pulse compressor is compensating for the GVD introduced by the pulse stretcher and amplifier chain.

\section{A.4. Ti:Sapphire-Based Regenerative Amplifier}

The S-polarized seed pulse train $(82 \mathrm{MHz})$ from the pulse stretcher is directed into the regenerative amplifier (see figure A.4.1). This regenerative amplifier is very similar to that built by Zhou et.al ${ }^{13}$. This amplifier includes a thin film polarizer (optimized at $1053 \mathrm{~nm}$ ) which transmits P-polarized light and ejects S-polarized light into a sequential amplifier chain. A pockel cell is included in the regenerative amplifier to switch a single seed pulse from S-polarization to $\mathrm{P}$ polarization at a $10 \mathrm{~Hz}$ rate. The polarizationswitched seed pulse then propagates through the amplifier cavity, undergoing gain on each pass through the Ti:Sapphire $\operatorname{rod}\left(\mathrm{L}=2.54 \mathrm{~cm}, \mathrm{~T}_{\text {rod }}=18^{\circ} \mathrm{C}\right) . \mathrm{A} \mathrm{Nd}: \mathrm{YAG}(532 \mathrm{~nm}, 5 \pm$ $0.5 \mathrm{~ns}, 95 \mathrm{~mJ}$, beam diameter $<2 \mathrm{~mm})^{14}$ laser optically pumps the Ti:Sapphire rod, and in the absence of the seed pulse, will cause free-running oscillation in the cavity. The large fluorescence bandwidth $(\lambda \mathrm{p} \cong 790 \mathrm{~nm}, \Delta \lambda \cong 180 \mathrm{~nm})$ and the long fluorescence lifetime $(\tau=3.2 \mu \mathrm{m})$ of Ti:Sapphire ${ }^{15}$ allow the rod to act as multi-pass gain medium while causing a minimum of spectral gain narrowing. After multiple passes, the amplified seed saturates 
the gain medium, and the seed amplitude maximizes. At this point, the pockel cell is then used to switch the amplified seed polarization back to S polarization, causing the amplified pulse to be ejected into the sequential amplifier chain. A quad-pulser (built at LLNL) energizes the pockel cell with a double pulse $(4 \mathrm{~ns}, 5 \mathrm{kV}$, pulse separation $\approx 1 \mu \mathrm{s}, 10$ $\mathrm{Hz}$ ) signal. The first electrical pulse injects the seed pulse into the amplifier and the second electrical pulse ejects the amplified pulse into the sequential amplifier chain. This double pulse, $10 \mathrm{~Hz}$ signal results in a $10 \mathrm{~Hz}, 5 \mathrm{~mJ}$ pulse output.

Unseeded and Seeded Regenerative Amplifier Operation

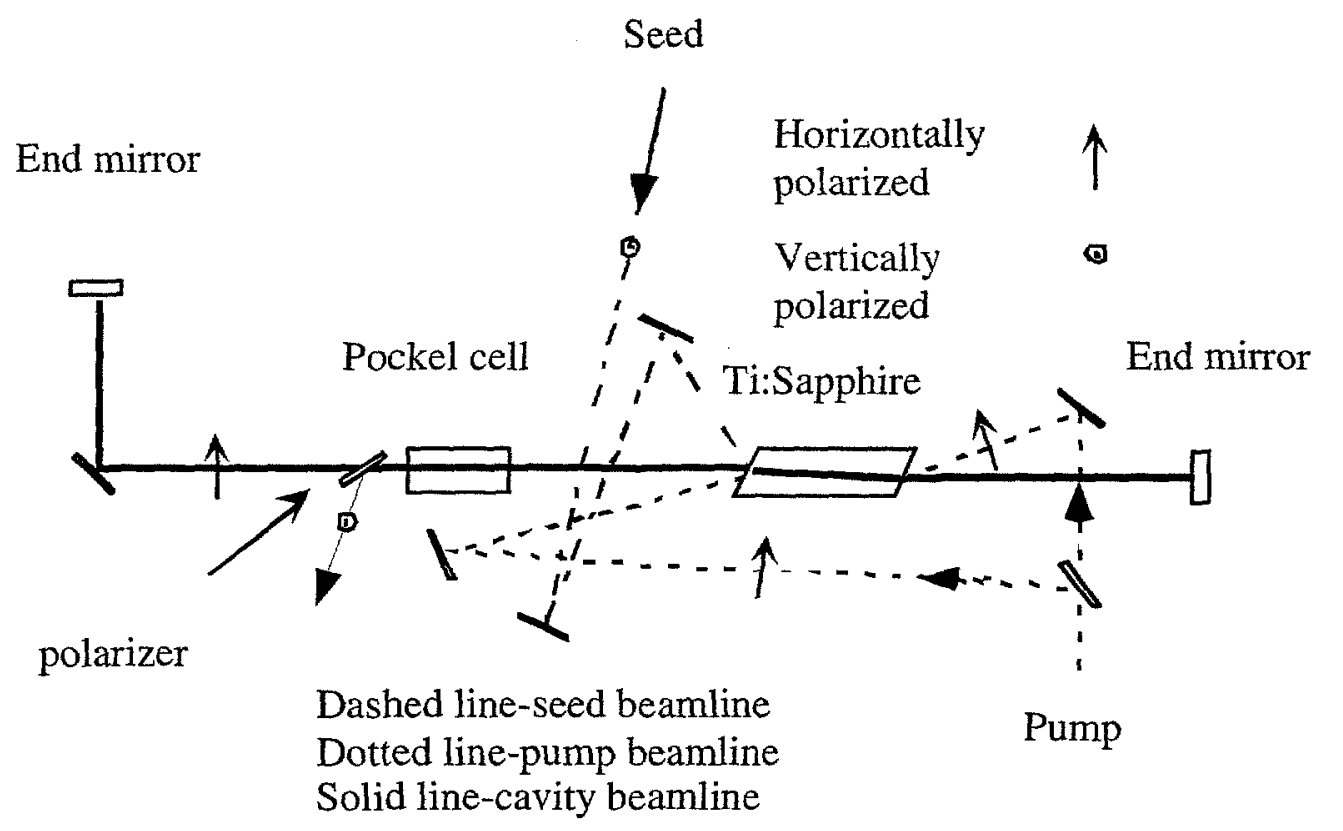

Figure A.4.1 Diagram of Ti:Sapphire-Based Regenerative Amplifier. The oscillator beam path, shown as a solid line, describes the cavity beam path.

\section{Unseeded Operation}

If one blocks the seed pulse train from entering the amplifier cavity, the cavity may still produce laser output. Unseeded, the regenerative amplifier is a cavity-dumped, Q- 
switched laser oscillator. When the pump power density supplied by the Nd:YAG exceeds the threshold pump power, P-polarized ASE laser oscillation occurs in the cavity. This oscillation starts by spontaneous emission in the Ti:Sapphire and then builds up as the signal is amplified by the Ti:Sapphire rod. This oscillation is affected by the operation of the pockel cell. When the first pulse of the double pulse signal from the quad pulser energizes the pockel cell, the P-polarized light is switched to S-polarization and is ejected. The cavity Q then drops to a low value (see Figure A.4.2).

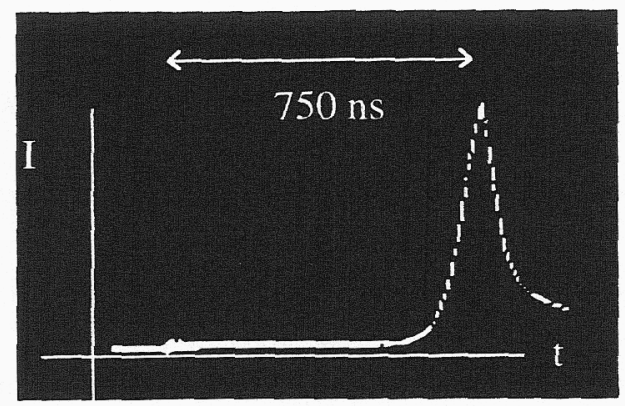

Figure A.4.2 Unseeded Regenerative Amplifier Leakage Signal. This signal originates from leakage through a cavity end mirror which is directed into a photodiode.

This 'de-Q-switching' prevents oscillation until after the pockel cell is de-energized. After the first pulse signal turns off, the polarizer ceases to reject energy, and the cavity can begin to build up laser oscillation to a maximum level. A typical free-running oscillation build-up time is $750 \mathrm{~ns}$, and after this maximum intensity is reached, gain saturation and cavity losses cause the intensity to drop.

\section{Seeded Operation}

Once injected into the Ti:Sapphire rod, the P-polarized seed pulse competes with noise photons for stimulated emission. One finds the peak noise power produced by a laser amplifier is $\frac{h v^{2}}{\lambda} \Delta \lambda$, where $\Delta \lambda$ is the supported bandwidth of the amplifier cavity ${ }^{16}$. 
This supported bandwidth is that of the Ti:Sapphire rod modified by the presence of the thin film polarizer and is approximately $15 \mathrm{~nm}$. This leads to a peak noise power of order $1 \mu \mathrm{W}$. The unamplified seed peak pulse power is approximately $1.7 \mathrm{~W}$, and if one assumes the first five round trips shown in Figure A.4.3 experience unsaturated smallsignal gain, then the coupling efficiency between the seed and the Ti:Sapphire rod can be estimated to be above $1 \%$. This gives an absorbed, unamplified seed peak power of $>200$ $\mu W$, far in excess of the noise power. Since the cavity rate equation ${ }^{17}$, $\frac{d n(t)}{d t}=K\left[N_{2}(t)-N_{1}(t)\right] n(t)-\gamma_{2}(t) n(t)$, where $n(t)$ in the photon density, $\mathrm{N}_{2}(\mathrm{t})$ and $\mathrm{N}_{1}(\mathrm{t})$ are the upper and lower population densities, respectively, $\mathrm{K}$ is the coupling constant, and $\gamma_{2}$ is the decay constant, shows that light amplification is proportional to the number of photons initially present in the system, the seed pulse would dominate the amplification process. The seed causes the regenerative amplifier to act like a multi-pass amplifier.

The seed passes through the gain medium repetitively (72 passes for a seed buildup time of $650 \mathrm{~ns}$ ), undergoing phase and amplitude modulation as it interacts with the gain medium. If one uses a linearly-chirped gaussian pulse to model the seed pulse, the spectrum of the electric field would be expressed below ${ }^{18}$.

$$
E(\omega)=\sqrt{\frac{\pi}{\Gamma_{0}}} \exp \left(\frac{-\left(\omega-\omega_{0}\right)^{2}}{4 \Gamma_{0}}\right)
$$

Here, the parameter, $\Gamma_{0}=a-i b$, describes the pulse duration and degree of linear chirp (where $\mathrm{a}$ and $\mathrm{b}$ are estimated for the $1 \mathrm{~ns}, 10.5 \mathrm{~nm}$ seed pulse to be $1.39 \times 10^{18} \mathrm{~s}^{-2}$ and $8.92 \times 10^{21} \mathrm{~s}^{-2}$, respectively). After one pass through the gain medium, the field would be modified as shown below. 


$$
E(\omega)=\sqrt{\frac{\pi}{\Gamma_{0}}} \exp \left(\frac{-\left(\omega-\omega_{0}\right)^{2}}{4 \Gamma_{0}}\right) \exp (\alpha L+i \beta L)
$$

where $\alpha=\alpha\left(\omega-\omega_{0}\right)$ is the frequency-dependent gain coefficient, $\beta=\beta\left(\omega-\omega_{0}\right)$ is the frequency-dependent phase shift, and $L$ is the length of the Ti:Sapphire $\operatorname{rod}(2.54 \mathrm{~cm})$.

In Ti:Sapphire, the fluorescence spectrum (FWHM $\Delta \lambda=180 \mathrm{~nm}$ ) can be described with a Lorentzian line shape, and the resulting frequency-dependent amplification is shown below.

$$
\alpha=\alpha_{m 0}-\alpha_{m 0}\left(\frac{\lambda^{2}}{\pi c \Delta \lambda}\right)^{2}\left(\omega-\omega_{0}\right)^{2}
$$

The stretcher in the master oscillator reduces the seed pulse to a bandwidth of $10.5 \mathrm{~nm}$ prior to injection into the amplifier, and in the Ti:Sapphire rod, the seed experiences negligible gain narrowing. One can then approximate the gain as constant with respect to frequency, and separate the effects of gain and phase shift, as shown below.

$$
E(t)=\frac{1}{\sqrt{2 \Gamma_{0}}} \exp \left(\alpha_{m o} L\right) \int_{-\infty}^{\infty} d \omega \exp (i \omega t) \exp \left(i \beta\left(\omega-\omega_{0}\right) L\right) \exp \left(\frac{-\left(\omega-\omega_{0}\right)^{2}}{4 \Gamma_{0}}\right)
$$

Initially, the seed fluence is too low to saturate the Ti:Sapphire rod. After multiple passes through the rod at low fluence, the seed pulse field is described by the expression below.

$$
E(t)=\exp \left(N \alpha_{m o} L\right) \int_{-\infty}^{\infty} d \omega \exp (i \omega t) \exp \left(-N \alpha_{L}(\omega)-i N \beta\left(\omega-\omega_{0}\right) L\right) \exp \left(\frac{-\left(\omega-\omega_{0}\right)^{2}}{4 \Gamma_{0}}\right)
$$

where $N$ is the number of passes through the gain medium and $\alpha_{L}(\omega)$ is the frequencydependent cavity losses produced by the thin-film polarizer, pockel cell, and end mirrors.

Initially, after the pump laser has excited the Ti:Sapphire rod, the seed pulse experiences exponential gain. However, the exponential gain coefficient, $\alpha_{\mathrm{m} 0}$, is proportional to the population inversion, and after the seed passes through the gain 
material, it depletes the population inversion by reducing the number of atoms in the upper energy level. This depletion reduces the gain, and the gain saturates as the inversion becomes completely depleted. After many round trips, the seed loses intensity as the gain medium fully saturates and the cavity losses diminish the pulse intensity. The plot of seed intensity vs time is shown in figure A.4.3 displays this sequence.

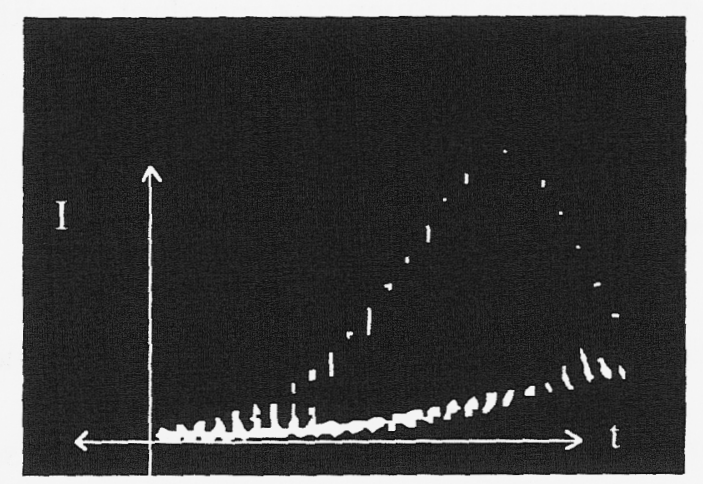

Figure A.4.3 Seeded Regenerative Amplifier Leakage Signal. One signal is shown in this plot, and it is composed of curve with spikes spaced at every $9 \mathrm{~ns}$. This signal originates from leakage through a cavity end mirror which is directed into a photodiode. The spikes shown are intensity spikes due to arrival of the amplified pulse at the end mirror.

This gain saturation behavior is seen in the expression of $\mathrm{G}_{\mathrm{pe}}$, the pulse-averaged field gain coefficient, shown below ${ }^{19}$.

$$
G_{p e}=\frac{\ln \left[\left(G_{0}-1\right) /\left(G_{f}-1\right)\right]}{\ln \left[\left(G_{0}-1\right) /\left(G_{f}-1\right)\right]-\ln \left[\frac{G_{0}}{G_{f}}\right]},(\mathrm{A} .11)
$$

where $G_{f}=\frac{G_{0}}{G_{0}-\left(G_{0}-1\right) \exp \left[\frac{-E_{\text {in }}}{E_{s}}\right]}, \mathrm{E}_{\mathrm{s}}$ is the saturation fluence for Ti:Sapphire, $\mathrm{E}_{\text {in }}$ is the input pulse fluence, and $G_{0}$ is the small-signal gain $\left(G_{0}=\exp \left(\alpha_{\mathrm{m} 0} L\right) \approx 1.244\right)$.

At low input pulse energy, this expression reduces to $G \cong G_{0}=\exp \left(\alpha_{m 0} L\right)$, while as input pulse fluence becomes larger than the saturation fluence, $E_{\mathrm{in}} / \mathrm{E}_{\mathrm{s}}>>1$, the above expression reduces to $G \approx 1+\left(\frac{E_{s}}{E_{\text {in }}}\right) \alpha_{\text {mo }} L$. With $(7)$, one estimates that the pulse average gain drops 
from a small signal gain, $G_{0}$, of 1.244 to a value of approximately 1 at maximum pulse energy of $5 \mathrm{~mJ}$.

The expression for the Ti:Sapphire-induced phase shift, $\exp \left(i \beta\left(\omega-\omega_{0}\right) \mathrm{L}\right)$, can be expanded by expanding $\beta\left(\omega-\omega_{0}\right)=\beta\left(\omega_{0}\right)+\beta^{\prime}\left(\omega_{0}\right)\left(\omega-\omega_{0}\right)+\beta^{\prime \prime}\left(\omega_{0}\right)\left(\omega-\omega_{0}\right)^{2} / 2$. After inserting this expansion into (6) and integrating, one finds the initial gamma parameter $\Gamma_{0}\left(\Gamma_{0}=a-\right.$ ib) of the pulse, after passing $\mathrm{N}$ times through the Ti:sapphire rod, becomes modified. This modified expression is shown below.

$$
\Gamma=\frac{a\left(1-2 N \beta^{\prime \prime}\left(\omega_{0}\right) b\right)+2 n \beta^{\prime \prime}\left(\omega_{0}\right) a b}{\left[\left(1-2 N \beta^{\prime \prime}\left(\omega_{0}\right) b\right)^{2}-\left(2 n \beta^{\prime \prime}\left(\omega_{0}\right) a\right)^{2}\right]}-i b \frac{\left(1-2 N \beta^{\prime \prime}\left(\omega_{0}\right) b+2 n \beta^{\prime \prime}\left(\omega_{0}\right) a\right)}{\left[\left(1-2 N \beta^{\prime \prime}\left(\omega_{0}\right) b\right)^{2}-\left(2 n \beta^{\prime \prime}\left(\omega_{0}\right) a\right)^{2}\right]}
$$

As seen in (7), after 72 passes through the regenerative amplifier, group velocity dispersion (GVD), which corresponds to the $\beta$ " term in the pulse phase expansion $\left(\beta^{\prime \prime} \approx 3.55 \times 10^{-32} \mathrm{~s}^{2}\right)$, does not significantly alter the temporal pulsewidth and phase of the pulse after amplification.

After the amplified pulse amplitude has maximized, the pockel cell is used to eject the amplified pulse into the Nd:Glass amplifier chain. This is done by selecting the proper separation time between the two pulses in the double pulse pockel cell signal. Since the first pockel cell signal pulse gates in the seed pulse, the second pulse ejects the pulse, and the separation time determines how much time the amplifier is allowed to amplify the seed pulse before ejection. As a practical matter, the switch-out time is often selected to be approximately $10 \mathrm{~ns}$ later in time than that of maximum amplified seed intensity. This is done such that any increase in seeded cavity build-up time will not cause large shot-to-shot variations in the output pulse intensity.

\section{A.5. Sequential Amplifier Chain}


After pulse switch-out in the regenerative amplifier, laser pulses propagate into the sequential amplifier chain(see Figure A.5.1). The chain includes three Nd:Glass amplifiers, and together these amplifiers increase laser pulse energy from $5 \mathrm{~mJ}$ to a maximum of $4 \mathrm{~J}$. Three pulse slicers are included in the chain. These pulse slicers, comprised by two cross-polarizers and a polarization-switching pockel cell, function as a fast-acting optical gates which attenuate any satellite pulses which leak out of the regenerative amplifier prior to or following laser pulse switch-out, as well as any ASE from the regenerative amplifier and the amplifiers in the chain. These pulse slicers reduce the intensity ratio of the satellite pulse and the switch-out pulse to $3 \times 10^{-10}$. Faraday rotators are used in combination with brewster-angle polarizing glass plates to prevent destructive back-reflections from the amplifier rods. To prevent excessive laser fluence in the amplifier chain, laser beam diameter is enlarged by vacuum spatial filters placed in front of each amplifier. Also, a serrated aperture is used in combination with these vacuum spatial filters to form a relay imaging system. This relay-imaging system produces a smooth 'flat-top' laser beam profile in the laser system, thereby suppressing laser-induced optical damage in high energy pulses. 


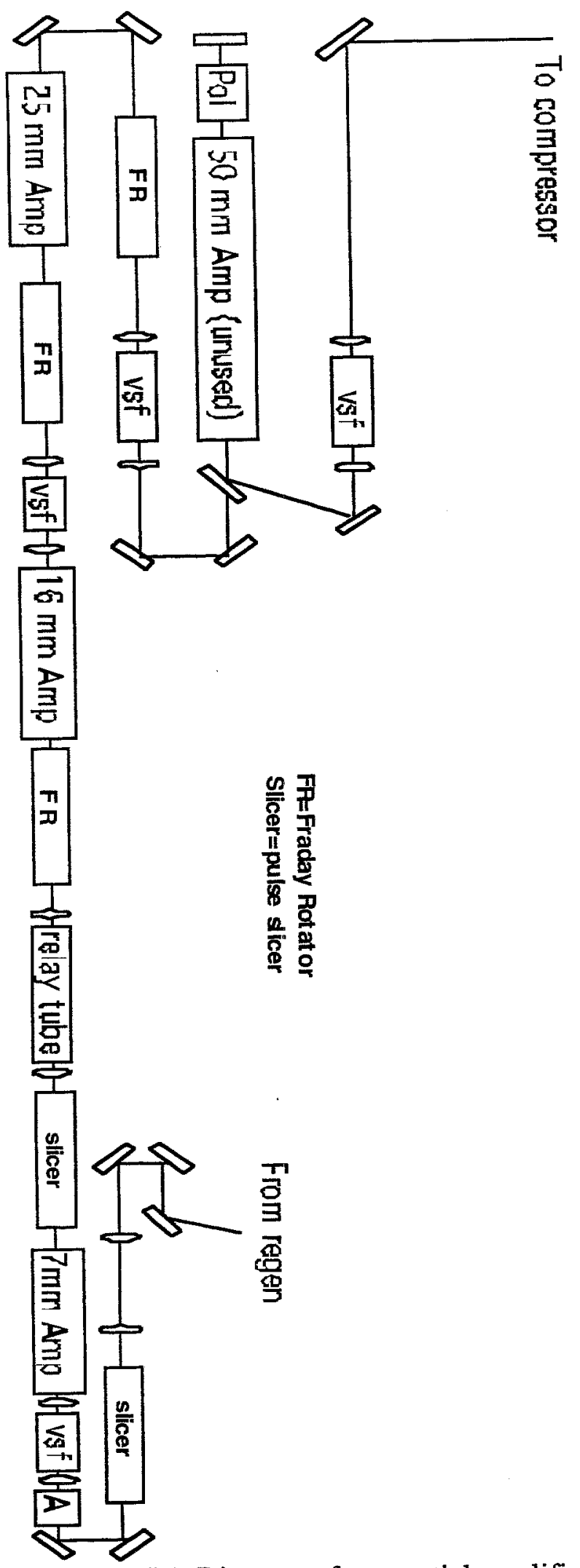

Figure A.5.1. Diagram of sequential amplifier chain. 


\section{Nd:Glass Amplifiers}

The backbone of the sequential amplifier chain is provided by three Nd:Glass solid state laser amplifiers. The $7 \mathrm{~mm}$ diameter amplifier is pumped by flash lamps energized by a $1.7 \mathrm{kV}$ electrical signal, and the amplifier increases the laser pulse energy from $5 \mathrm{~mJ}$ to $15 \mathrm{~mJ}$. After expansion, the beam passes into the $16 \mathrm{~mm}$ amplifier. The amplifier is also pumped by flash-lamps, and the flashlamp voltage is varied from $1.1 \mathrm{kV}$ to $2.1 \mathrm{kV}$ in order to vary the laser rod gain. At maximum flash lamp voltage, the rod amplifies the pulse from $15 \mathrm{~mJ}$ to $400 \mathrm{~mJ}$. Similarly, the $25 \mathrm{~mm}$ amplifier is pumped by flash lamps receiving a voltage range from $1.1 \mathrm{kV}$ to $2.1 \mathrm{kV}$. The $25 \mathrm{~mm}$ amplifier, at maximum flash lamp voltage, amplifies the pulse from $400 \mathrm{~mJ}$ to $4 \mathrm{~J}$. Each flash lamp signal is synchronized to the regenerative amplifier $10 \mathrm{~Hz}$ output pulse train such that the time of maximum population inversion coincides with arrival of the laser pulse at the amplifier. Each amplifier is water-cooled, and to avoid high temperature birefringence in the laser rods, the amplified pulse firing rate must be kept below one shot every three minutes.

As the laser pulse passes through each amplifier, it undergoes not only laser amplification but also gain narrowing and frequency-dependent phase shifts, and these effects can negatively affect the quality of the output laser pulse. To describe the effect of amplification, the amplification and phase modulation effects are calculated on a linearly chirped pulse. For simplicity, one assumes a gaussian temporal pulse shape which is described by the expressions below.

$$
E(r, x, t)=E_{0} \exp \left(-i \omega t+i k x-\Gamma_{0} t^{2}\right)
$$




$$
E(r, x, \omega)=\sqrt{\frac{\pi}{\Gamma_{0}}} E_{0} \exp \left(i k x-\frac{\left(\omega-\omega_{0}\right)^{2}}{4 \Gamma_{0}}\right)
$$

where $\mathrm{k}$ is the incident wave vector, $\mathrm{x}$ is the distance along the propagation axis, and $\Gamma_{0}$ $=a-i b$ is a complex constant, with a determining the gaussian pulsewidth and $b$ determining the amount of linear chirp on the pulse.

At the end of the amplifier rod, the waveform is described by the following expression:

$$
E(x, \omega)=\sqrt{\frac{\pi}{\Gamma_{0}}} E_{0} \exp \left(i k(\omega) L-\frac{\left(\omega-\omega_{0}\right)^{2}}{4 \Gamma_{0}}+\alpha\left(\omega, J_{\text {in }}\right) L\right)
$$

where $\mathrm{k}=\mathrm{k}_{0}+\mathrm{k}^{\prime}\left(\omega-\omega_{0}\right)+\mathrm{k}^{\mathrm{n} \prime}\left(\omega-\omega_{0}\right)^{2} / 2$ and $\alpha\left(\omega, \mathrm{J}_{\text {in }}\right)$ is the frequency-dependent, input fluencedependent gain coefficient.

Two important aspects of the gain are its dependence on frequency and also on incident laser fluence. The frequency dependence of the gain coefficient, $\alpha\left(\omega, \mathrm{J}_{\mathrm{in}}\right)$, is determined by the fluorescence spectrum of $\mathrm{Nd}$ :glass, and in the first approximation, the gain coefficient can be described by the expression below ${ }^{20}$.

$$
\alpha(\omega)=\alpha_{m o}-\alpha_{m}\left(\frac{2}{\Delta \omega_{a}}\right)^{2}\left(\omega-\omega_{0}\right)^{2}
$$

where $\Delta \omega_{\mathrm{a}}$ is the bandwidth of the Nd:glass (phosphate glass, $\Delta \omega_{\mathrm{a}} \cong 2.29 \times 10^{14} \mathrm{~s}^{-1}$ )

If one substitutes (4) into (3), one can then observe the bandwidth losses caused by the limited fluorescence bandwidth in the Nd:glass. This expression can be shown as a function of wavelength and is shown below.

$$
\left(\frac{1}{\Delta \lambda_{f}}\right)^{2}=\left(\frac{1}{\Delta \lambda_{i}}\right)^{2}+\ln G\left(\frac{1}{\Delta \lambda_{\text {Nd:glass }}}\right)^{2}
$$


where $\Delta \lambda_{\mathrm{F}}$ is the bandwidth of the output pulse, $\Delta \lambda_{i}$ is the bandwidth of the input pulse, and $\Delta \lambda_{\text {Nd:glass }}$ is the bandwidth of the Nd:glass phosphate amplifier rod $(21.9 \mathrm{~nm})^{21}$.

After pulse switch-out in the regenerative amplifier, each pulse is linearly-chirped and has $10.5 \mathrm{~nm}$ (FWHM) of bandwidth and $5 \mathrm{~mJ}$ energy. After maximum amplification of the pulse through the entire amplifier chain, each laser pulse has $4 \mathrm{~J}$, and the pulseaveraged gain through the amplifier chain, G, is approximately 800 . Given this gain and the input bandwidth, one finds from (5) that the final bandwidth, $\Delta \lambda_{\mathrm{f}}$, is $6.59 \mathrm{~nm}$. This compares favorably with the observed final bandwidth of $5 \mathrm{~nm}$, especially since the actually laser pulse shape is not gaussian but of the functional form $\operatorname{sech}^{2}(t)$. Incident laser fluence can also have a limiting effect on beam amplification. In high energy laser systems such as the picosecond laser system, incident laser fluences are high enough to saturate the laser amplifiers. One would then expect reduced amplifier gain. In this case, however, Nd:glass amplifiers has a saturation fluence ${ }^{22}$ of $2-5 \mathrm{~J} / \mathrm{cm}^{2}$, while the maximum laser fluence present in the amplifiers is less than $1 \mathrm{~J} / \mathrm{cm}^{2}$. In this case, one would not expect gain saturation to affect the pulse-averaged gain. Gain saturation would also be reduced by the fact that the incident pulse is linearly chirped. As a function of time, the center pulse frequency will shift. In this case, the gain saturation caused by the leading edge of the laser pulse would have a reduced effect on the trailing elements of the pulse since the pulse spectrum of the leading and trailing edges do not completely overlap.

Propagation through the Nd:glass rod also causes phase shifts in the laser pulse. Combining (3) and (4), one can find that the total expression for the waveform is the following: 


$$
\begin{aligned}
& E(r, x, \omega)=\sqrt{\frac{\pi}{\Gamma_{0}}} E_{0} \exp \left(i k_{0} L+i k^{\prime}\left(\omega-\omega_{0}\right) L\right. \\
& \left.+i \frac{k^{\prime \prime}}{2}\left(\omega-\omega_{0}\right)^{2} L-\frac{\left(\omega-\omega_{0}\right)^{2}}{4 \Gamma_{0}}+\alpha_{m o} L-\alpha_{m}\left(\frac{2}{\Delta \omega_{a}}\right)^{2}\left(\omega-\omega_{0}\right)^{2} L\right)
\end{aligned}
$$

Recalling that $\Gamma_{0}=\mathrm{a}-\mathrm{ib}$ and grouping the exponential argument into powers of frequency, one sees that the phase can be expressed by the following:

$$
\begin{aligned}
& E(r, x, \omega)=\sqrt{\frac{\pi}{\Gamma_{0}}} E_{0} \exp \left(i k_{0} L+i k^{\prime} L\left(\omega-\omega_{0}\right)+\alpha_{m 0} L\right. \\
& \left.+i\left(\frac{k^{\prime \prime}}{2} L-\frac{b}{4\left(a^{2}+b^{2}\right)}\right)\left(\omega-\omega_{0}\right)^{2}-\left(\alpha_{m}\left(\frac{2}{\Delta \omega_{a}}\right)^{2} L+\frac{a}{4\left(a^{2}+b^{2}\right)}\right)\left(\omega-\omega_{0}\right)^{2}\right)
\end{aligned}
$$

The quadratic phase coefficient of the output pulse, $k " L / 2-b /\left(4\left(a^{2}+b^{2}\right)\right)$, differs from that of the input pulse by the addition of the GVD term, $\mathrm{k}$ "L/2 (where $\mathrm{k}^{\prime \prime}=2.5456 \times 10^{-32} \mathrm{~s}^{-2}$ ). This addition affects the amount of linear chirp on the laser pulse, and during recompression, can cause undesirable pulse features to develop since the pulse compressor will not be able to exactly compensate for this GVD and the total stretcherinduced phase shift.

\section{Pulse Slicers}

Leakage or satellite pulses preceding or following the main pulse in the sequential amplifier chain can, if allowed to pass through the amplifier chain and into the target chamber, preheat the laser target prior to arrival of the main ablation pulse. Specifically, the vast majority of seed pulses in the $82 \mathrm{MHz}$ pulse train directed into the regenerative amplifier are rejected by the polarizer and propagate through the amplifier chain. Additionally, a small portion of the amplified pulse can leak out of the amplifier prior to 
switchout, and this pulse can precede to main amplifier pulse into the amplifier chain. Both types of satellite pulses can propagate into the target chamber, caused target preheating prior to arrive of the main pulse. Target preheat can cause undesirable target breakdown and plasma expansion. Pulse slicers are included in the amplifier chain to attenuate these satellite pulses and avoid preheat. Each pulse slicer acts as an optical switch with a rise time on order $1 \mathrm{~ns}$. Driven by a $10 \mathrm{~Hz}, 5 \mathrm{kV}, 4 \mathrm{~ns}$ electrical signal, the pockel cell in the pulse slicer allows the main pulse to pass unattenuated, and the satellite pulses arriving before or after the electrical signal are extinguished by the cross-polarizer pair.

Inserted between the two cross-polarizers is a $\mathrm{KH}_{2} \mathrm{PO}_{4}(\mathrm{KDP})$-based pockel cell. When energized by a high voltage electrical signal, the KDP crystal undergoes a linear electro-optic effect and becomes birefringent. The KDP crystal is orientated such that, when energized, the principle axis of the crystal are rotated $45^{\circ}$ from the polarization axis of the input polarizing prism. Along these principle axis the index of refraction are described below ${ }^{23}$.

$$
\begin{array}{r}
\mathrm{x}^{\prime} \mathrm{n}=\mathrm{n}_{0}-\mathrm{n}_{0}{ }^{3} \mathrm{r}_{63} \mathrm{E}_{\mathrm{z}} / 2=\mathrm{n}_{0}-\mathrm{n}_{0}{ }^{3} \mathrm{r}_{63} \mathrm{~V} /(2 \mathrm{~L}) \\
\mathrm{y}^{\prime} \mathrm{n}=\mathrm{n}_{0}+\mathrm{n}_{0}{ }^{3} \mathrm{r}_{63} \mathrm{E}_{\mathrm{z}} / 2=\mathrm{n}_{0}+\mathrm{n}_{0}{ }^{3} \mathrm{r}_{63} \mathrm{~V} /(2 \mathrm{~L})
\end{array}
$$

where $r_{63}$ is an element of the electro-optic tensor, $V$ is the longitudinally-applied voltage, $\mathrm{L}$ is the longitudinal length of the crystal, and $n_{0}$ is the unperturbed index of refraction.

This birefringence causes a relative phase difference between the $x^{\prime}$ and $y^{\prime}$ electric field components at the output of the pockel cell. If the first polarizing prism is set to pass vertically-polarized light, then the optical signal entering the pockel cell is described below. 


$$
\vec{E}_{\text {input }}=A_{0} \exp (i \omega t-i k z) \hat{y}
$$

Along the principle axis of the pockel cell, the field is described below.

$$
\begin{aligned}
& E_{y^{\prime}}=\frac{A_{0}}{\sqrt{2}} \exp (i \omega t-i k z) \hat{y} \\
& E_{x^{\prime}}=\frac{A_{0}}{\sqrt{2}} \exp (i \omega t-i k z) \hat{x}^{\prime}
\end{aligned}
$$

While energized by the high-voltage signal, the field at the output of the pockel cell is expressed by the following:

$$
\begin{aligned}
& E_{y^{\prime}}=\frac{A_{0}}{\sqrt{2}} \exp \left(i \omega t-i \frac{\omega}{c}\left(n_{0}+\frac{n_{0}^{3}}{2} r_{63} V\right)\right) \hat{y}^{\prime} \\
& E_{x^{\prime}}=\frac{A_{0}}{\sqrt{2}} \exp \left(i \omega t-i \frac{\omega}{c}\left(n_{0}-\frac{n_{0}^{3}}{2} r_{63} V\right)\right) \hat{x}^{\prime}
\end{aligned}
$$

These two components have a phase difference which is expressed below.

$$
\Gamma=\varphi_{x^{\prime}}-\varphi_{y^{\prime}}=\omega n_{0}^{3} r_{63} V / c
$$

$V$ is chosen such that $\Gamma=\pi$ and the output field, $E^{\prime}=E_{x^{\prime}}+E_{y^{\prime}}$ is vertically polarized along the polarization axis of the second polarizing prism. From (A.24), this value is calculated to be $6.58 \mathrm{kV}$. The three pulse slicers included in the picosecond laser system yield a intensity contrast ratio between main and satellite pulse of $3 \times 10^{-10}$. With this contrast ratio, undesirable laser target preheat due to satellite pulses can be safely neglected from LIBA analysis. 
Faraday Rotators

Since the beam is expanded and amplified by vacuum spatial filters along the length of the amplifier chain to prevent excess laser fluence from damaging the laser optics, one must be careful to prevent back reflections from counter propagating back into the amplifier chain. Any back reflected pulses would be amplified and reduced in spatial size during counter-propagation, becoming highly intense and a danger to the optical elements. To prevent back-reflections, faraday rotators are used to optically isolate each amplifier in the amplifier chain (see Figure A.5.2). As the laser pulse passes through the chain, it propagates through a brewster-angle reflecting polarizer and into the faraday rotator. This rotator rotates the linear polarization of the pulse $45^{\circ}$ as it passes through the rotator medium. Any back reflections will back-propagate through the medium, and the polarization will again rotate another $45^{\circ}$. The back reflected pulse is now polarized $90^{\circ}$ with respect to the reflecting polarizer, and the pulse is reflected off axis into a beam stop.

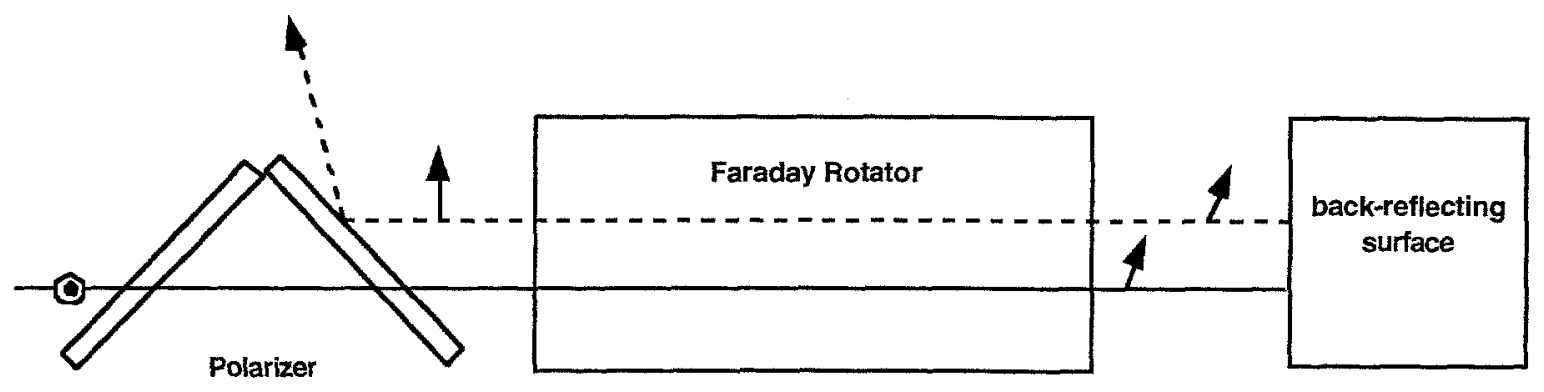

Figure A.5.2. The solid line represents the forward-travelling wave, while the dotted-line represents the counter-propagating wave which is rejected by the polarizer. Arrows show pulse polarization is initially vertical, then $45^{\circ}$ off vertical after passing through the faraday rotator. The back-reflecting pulse is rotated again $45^{\circ}$, and the arrows show a horizontal polarization after the back-reflection passes through the rotator. 
The faraday rotator is a magnetically-sensitive medium such as TGG (terbiumgallium-garnet) or YIG (yttrium-iron-garnet) surrounded by a permanent magnet. This magnet generates a magnetic field along the longitudinal axis of the medium. This field causes the medium to develop circularly-polarized normal modes with different indices of refraction. These indices are described below.

$$
\begin{aligned}
& n_{+}=\sqrt{n_{0}^{2}+\gamma B} \\
& n_{+}=\sqrt{n_{0}^{2}-\gamma B}
\end{aligned}
$$

Propagation through this medium then causes a net rotation of the polarization vector.

This rotation is described below ${ }^{24}$.

$$
\Theta=\rho L=- \text { VHL (A.26) }
$$

where $H$ is the magnetic field, $V$ is the Verdet coefficient $(V=-20 \mathrm{rad} / \mathrm{T} \mathrm{m}$ at $1060 \mathrm{~nm})$, and $\mathrm{L}$ is the length of the faraday rotator $(63 \mathrm{~mm})$.

By selecting $B$ to be $0.62 \mathrm{~T}$, the faraday rotator will rotate the pulse polarization $\Theta=\pi / 4$.

Back reflections which counter-propagate through the faraday rotator undergo an additional polarization rotation of $\Theta=\pi / 4$ in the same rotational sense, since the faraday effect is independent of direction of the laser pulse wave vector, $k$. After undergoing $\Theta=\pi / 2$ polarization rotation, the brewster angle polarizer will not transmit the laser pulse, thereby preventing the back-reflecting from propagating up the chain. In this way, the faraday rotator, in combination with the brewster-angle polarizer, acts as an optical isolator. 
The vacuum spatial filters included in the sequential amplifier chain are each composed of two lenses $\left(f_{1}, f_{2}\right)$ separated by a distance equal to their combined focal length $\left(d=f_{1}+f_{2}\right)$. As the laser pulse propagates through the first lens, it is focused into a new beam pattern. At the position of best focus, this beam pattern is the spatial Fourier transform of the incident beam. Positioned at best focus is a hard-edged pinhole. This pinhole blocks the high-frequency spatial components of the pulse, and only the low frequency components of the pulse are free to propagate into the second lens. The combination of the two lenses and the pinhole removes high frequency spatial features, such as dust spots, diffraction patterns, and other beam irregularities, from the laser pulse. This helps reduce hot spots in the beam and prevent optical damage in the laser system.

The use of serrated apertures with spatial filtering serves to apodize the effect of spatial filtering ${ }^{25}$. In general, the serrated aperture is placed in front of the input lens of the spatial filter. In the absence of a serrated aperture, the edge of the lens becomes the relevant aperture. In either case, the aperture is described by an aperture or pupil function, $P$. The effect of the lens on the spatial frequency spectrum of the incident pulse is described by $\mathrm{H}$, the amplitude transfer function. The transfer function can be written as a scaled pupil function, $H=P\left(\lambda f f_{x}, \lambda f f_{y}\right)$, where $\lambda$ is the central wavelength of the laser and $f$ is the focal length of the first lens. In the case of the lens edge being the relevant aperture, the pupil function, $\mathrm{P}$, can be described by the expression below.

$$
P(x, y)=\begin{aligned}
& 1 \text { for } \sqrt{x^{2}+y^{2}} \leq \text { lens radius, } R \\
& 0 \text { for } \sqrt{x^{2}+y^{2}}>\text { lens radius, } R
\end{aligned}
$$

Here, $x=\lambda f f_{x}$ and $y=\lambda f f_{y}$. In the case of a serrated aperture, one can use a generalized pupal . function, $\mathrm{P}^{\prime}$, which can be written as; 


$$
P^{\prime}(x, y)=P(x, y) e^{i k W(x, y)}(\mathrm{A} .28)
$$

where $W(x, y)$ is an effective path length adjustment and may be complex.

Using P', one can write $\mathrm{H}^{\prime}$ for a coherent laser pulse as;

$$
H^{\prime}=P^{\prime}\left(\lambda f f_{x}, \lambda f f_{y}\right)(\mathrm{A} .29)
$$

$\mathrm{H}$ ' has the effect of further suppressing the off-axis spatial frequency components beyond that associated with $\mathrm{H}$ alone. This apodization enhances the spatial filtering function and results in a more uniform spatial beam profile.

${ }^{1}$ W. Koechner, Solid-State Laser Engineering (Springer, New York, 1996), p.76.

${ }^{2}$ A. Siegman, "Lasers", p. 243-263.

${ }^{3}$ Tbid., p. 1041-1050.

${ }^{4}$ Spectra-Physics, "Tsunami: Mode-locked Ti:sapphire Laser User's Manual", p. 3-4.

${ }^{5}$ Ibid., p. 3-2.

${ }^{6}$ Ibid., p. 4-9.

${ }^{7}$ A. Siegman, "Lasers", p. 1049-1050.

${ }^{8}$ Spectra-Physics, "Tsunami: Mode-locked Ti:sapphire Laser User's Manual", p.3-3.

${ }^{9}$ Ibid., p. 1056-1057.

${ }^{10}$ Ibid., p. 1129-1170.

${ }^{11}$ Ibid., p. 1129-1170.

${ }^{12}$ Ibid., p. 552-555.

${ }^{13}$ F. Zhou, I.P. Mercer, M.H.R. Hutchinson, C.N. Danson, and C.B. Edwards, Electronics. Letts. 1995, pp. 1060-1061.

${ }^{14}$ Spectra-Physics, "Pulsed Nd:YAG lasers User's Manual", p. 1-13.

${ }^{15}$ W. Koechner, "Solid-State Laser Engineering", p.77.

${ }^{16}$ A. Yariv, "Quantum Electronics", p.575.

${ }^{17}$ A. Seigman, "Lasers", p. 498.

${ }^{18}$ Ibid., p.331-361.

${ }^{19}$ Ibid., p.368-371.

${ }^{20}$ A. Seigman, "Lasers", p.359.

${ }^{21}$ W. Koechner, "Solid-State Laser Engineering", p.55.

${ }^{22}$ Ibid., p.160.

${ }^{23}$ A. Yariv, "Quantum Electronics", pp.310-314.

${ }^{24}$ Newport product catalog, I-2.

${ }^{25} \mathrm{~J}$.W. Goodman, Introduction to Fourier Optics, pp.126-165. 


\section{APPENDIX B:SINGLE-SHOT INTENSITY AUTOCORRELATORS}

Laser pulse duration has a strong effect on laser/matter interaction dynamics and direct measurement of the laser pulse duration is a critical task. However, conventional measurement techniques such as photodiodes cannot resolve pulse durations in the picosecond and sub-picosecond range. Instead, the experimenter must rely on more elaborate diagnostics. In this experiment, I use single-shot, second harmonic generation (SHG), intensity autocorrelation to measure the laser pulsewidth. Single-shot intensity autocorrelations are obtained by splitting a sample of the compressed laser pulse into two beams and crossing the beams together in a KDP crystal. Crossing the two beams at the proper phase matching angle causes the KDP crystal to produce a $2 \omega$ signal which, when integrated by a Redicon linear detector array placed behind the KDP crystal, represents the intensity autocorrelation of the input laser pulse. By measuring the Full Width at Half-Maximum (FWHM) of this autocorrelation and using prior knowledge ${ }^{i}$ of the laser pulse shape, one can obtain the FWHM of the actual laser pulse.

In the autocorrelator (see Figure B.1), the input laser pulse is passed through a half-wave plate to rotate the polarization to the vertical orientation. The pulse is then divided into two daughter pulses. One daughter pulse is reflected from two mirrors and then through the KDP crystal at an angle, a, from the normal of the surface. The other pulse passes through a delay arm, off another mirror and into the KDP crystal at the angle -a. The delay arm is set such that the two beam paths are the same length, and the electric field in the KDP crystal can be described by the expression below.

$$
E(x, z, t)=E_{1}(x, z, t)+E_{2}(x, z, t)
$$




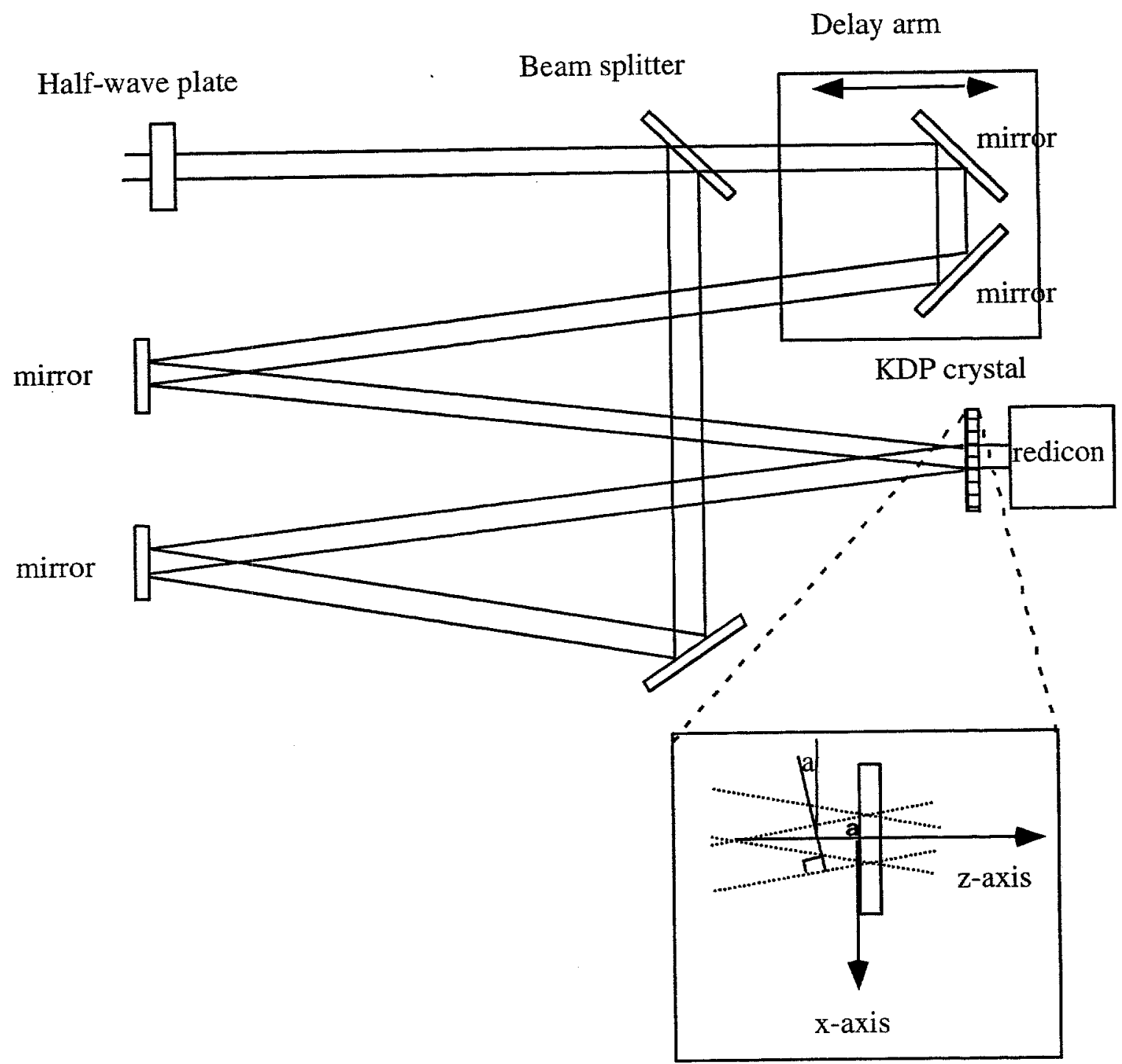

Figure B.1. Diagram of single-shot SHG-based autocorrelator.

The finite crossing angle, a, introduces a relative phase delay between the two daughter pulses at each point on the $\mathrm{x}$-axis. The mapping of delay on to the $\mathrm{x}$-axis is given as 
$\Delta t=2 x^{*} \tan (a) / c$, and for an input pulse with a flat-top beam profile, the incident field in the KDP crystal can be described by the expression below.

$$
E(t)=E(t-x \tan (a) / c)+E(t+x \tan (a) / c)
$$

Second harmonic generation in the KDP crystal is a non-linear function of the incident laser intensity, and the intensity of the second-harmonic signal is described below ${ }^{\text {ii }}$.

$$
I_{S H G}=2 \sqrt{\frac{\mu_{0}}{\varepsilon}} \omega^{2}\left(d_{i j k}\right)^{2} I\left(t-\frac{x}{c} \tan (a)\right) I\left(t+\frac{x}{c} \tan (a)\right) \frac{\sin ^{2}\left(\frac{\Delta k L}{2}\right)}{\left(\frac{\Delta k L}{2}\right)^{2}}
$$

where $\mathrm{L}$ is the length of the KDP crystal, $\Delta \mathrm{k}$ is the phase matching angle and $\mathrm{d}_{\mathrm{ijk}}$ is the non-linear optical susceptibility coefficient.

Since the redicon integrates the $\mathrm{I}_{\mathrm{SHG}}$ signal over a timescale of order milliseconds, the total effective signal read by the redicon at each $\mathrm{x}$-axis position is proportional to an intensity autocorrelation. This can be shown by the following:

$$
\begin{aligned}
& I_{S I G}(x)=\int_{-\infty}^{\infty} I_{S H G} d t \propto \int_{-\infty}^{\infty} I\left(t-\frac{x}{c} \tan (a)\right) I\left(t+\frac{x}{c} \tan (a)\right) d t= \\
& \int_{-\infty}^{\infty} I(t) I\left(t+\frac{2 x}{c} \tan (a)\right) d t \quad(B .4)
\end{aligned}
$$

With a known pulse shape, the FWHM of the autocorrelation can used to find the FWHM of the actual pulse. The picosecond laser produces pulses with a waveform which follows the $\operatorname{sech}^{2}(t)$ profile, and the ratio of the FWHM for a $\operatorname{sech}^{2}(t)$ pulse to its autocorrelation is $1 / 1.543^{\mathrm{iii}}$.

If, however, the input pulse shape is not known, this method will not necessarily produce reliable results. Since it is an intensity autocorrelation, the signal is intrinsically 
symmetriciv $^{\mathrm{iv}}$, and any temporal asymmetries in the pulse will not be shown in the autocorrelation. Also, the intensity autocorrelation does not show phase information, and it cannot directly measure the linear chirp of an input pulse. While this could present difficulties is eliminating pulse chirp, one can simply reduce chirp by reducing pulse duration. At minimum pulsewidth, the laser pulse has negligible linear chirp, and by adjusting the grating separation in the pulse compressor ("tuning the compressor") such as to minimize the pulsewidth, one can remove the linear chirp.

' J. Dunn, B.K.F. Young, A.K. Hankla, A.D. Conder, W.E. White, and R.E. Stewart, Proceedings of the $12^{\text {th }}$ International Conference of Laser Interaction and Related Plasma Phenomena, (S.Nakai and G.H. Miley, ed.), p.652.

"A. Yariv, "Quantum Electronics", p.393.

iii J. Diels, J. Fontaine, I. McMichael, and F. Simoni, Appl. Opt. 24, 1270 (1985).

${ }^{i v}$ J. Diels, "Ultrashort Laser Pulse Phenomena", p.366-367. 